\title{
APT Blanket System Model Based on Initial Conceptual Design - Integrated 1D TRAC System Model
}

by

L. L. Hamm

Westinghouse Savannah River Company

Savannah River Site

Aiken, South Carolina 29808

S. Y. Lee

M. A. Shadday

F. G. Smith, III

This paper was prepared in connection with work done under the above contract number with the U.S. Department of Energy. By acceptance of this paper, the publisher and/or recipient acknowledges the U.S. Government's right to retain a nonexclusive, royalty-free license in and to any copyright covering this paper, along with the right to reproduce and to authorize others to reproduce all or part of the copyrighted paper. 


\section{APT BLANKET SYSTEM MODEL BASED ON INITIAL CONCEPTUAL DESIGN -}

\section{Integrated 1D TRAC System Model}

L. Larry Hamm

Si Young Lee

M. Andy Shadday

Frank G. Smith, III

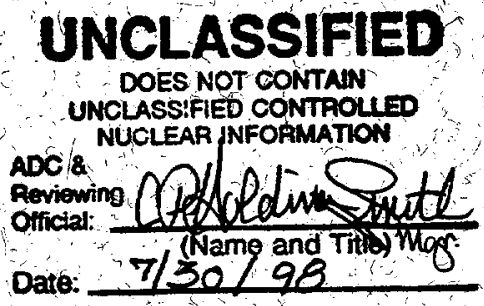

Westinghouse Savannáh River Company Savannah River Site Aiken, SC 29808

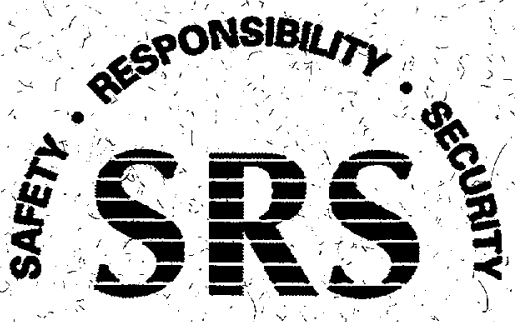




\section{DISCLAIMER}

This report was prepared as an account of work sponsored by an agency of the United States Government. Neither the United States Government nor any agency thereof, nor any of their employees, makes any warranty, express or implied, or assumes any legal liability or responsibility for the accuracy, completeness, or usefulness of any information, apparatus, product, or process disclosed, or represents that its use would not infringe privately owned rights. Reference herein to any specific commercial product, process, or service by trade name, trademark, manufacturer, or otherwise does not necessarily constitute or imply its endorsement, recommendation, or favoring by the United States Government or any agency thereof. The views and opinions of authors expressed herein do not necessarily state or reflect those of the United States Government or any agency thereof.

This report has been reproduced directly from the best available copy.

Available to DOE and DOE contractors from the Office of Scientific and Technical Information, P.O. Box 62, Oak Ridge, TN 37831; prices available from (615) 576-8401.

Available to the public from the National Technical Information Service, U.S. Department of Commerce; 5285 Port Royal Road, Springfield, VA 22161. 


\section{DISCLAIMER}

Portions of this document may be illegible in electronic image products. Images are produced from the best available original document. 


\section{WSRC-TR-98-0053}

KEYWORDS:

Accelerator Production of Tritium Blanket System

Conceptual Design TRAC Code

System Model

Safety Analysis

RETENTION - Permanent

\section{APT BLANKET SYSTEM MODEL BASED ON INITIAL CONCEPTUAL DESIGN -}

\section{Integrated 1D TRAC System Model}

SAVANNAH RIVER TECHNOLOGY CENTER

L. Larry Hamm

Si Young Lee

M. Andy Shadday

Frank G. Smith, III

Publication Date: July 1998

Westinghouse Savannah River Company

Savannah River Site

Aiken, SC 29808

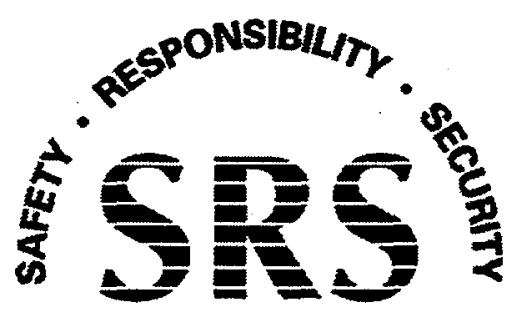

Prepared for the U.S. Department of Energy under Contract No. DE-AC09-96SR18500 
DOCUMENT: WSRC-TR-98-0053

TITLE: APT BLANKET SYSTEM MODEL BASED ON INITIAL CONCEPTUAL DESIGN - Integrated 1D TRAC System Model

\section{APPROVALS}

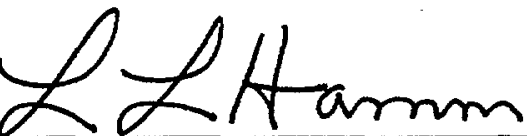

L. Larry Hamm, Task Leader (EM\&S Group/SRTC)

Date: $7-16-98$

Hotowy be.

Si Young Lee, Co-author (EM\&S Group/SRTC)

Date: $7-20-98$

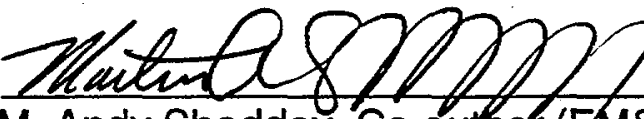

M. Andy Shadday, Co-author (EM\&S Group/SRTC)

Date: $7 / 20 / 98$

Frank I Lmeth 17

Frank G. Smith, III, Co-author (PC\&C Group/SRTC)

Date:

$7 / 20 / 98$

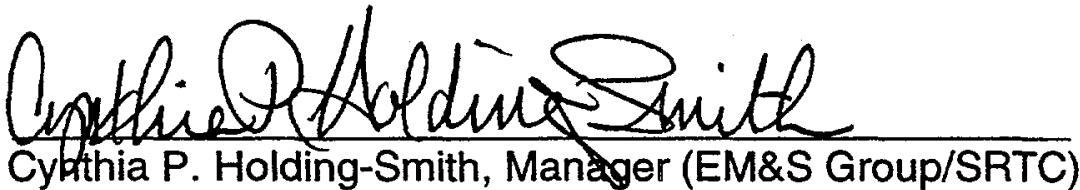

Date: $7 / 16 / 98$

m. Q. EBra

Martha A. Ebra, Manager (EDS/SRTC)

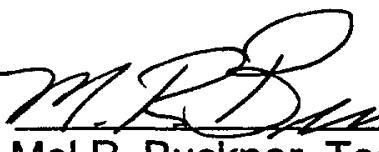

Mel R. Buckner, Technical \& Regulatory Lead (APT OPO)

The internal technical review function is being performed at the APT project level and is coordinated through LANL.

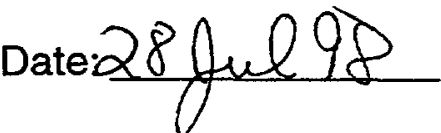

Date: $7 / 21 / 98$ 


\section{Table of Contents}

1 Introduction $\quad 1-1$

1.1 Overview of Blanket System .......................................................................... 1-1

1.2 Modeling Strategy ..................................................................................... 1-2

1.3 Analysis Requirements of Integrated System Model............................................ 1-3

1.3.1 System Model Requirements ................................................................ 1-3

1.3.2 System Code Selection ................................................................... 1-4

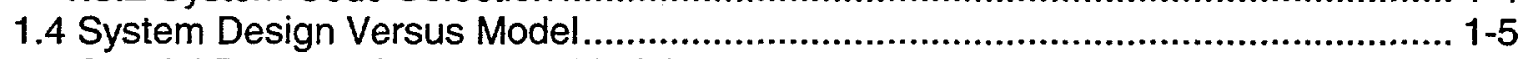

1.5 Special Purpose Standalone Models ............................................................. 1-6

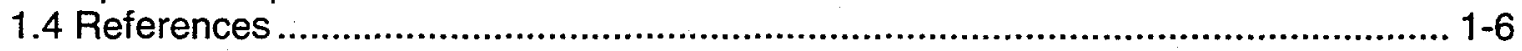

2 Module Descriptions $\quad$ 2-1

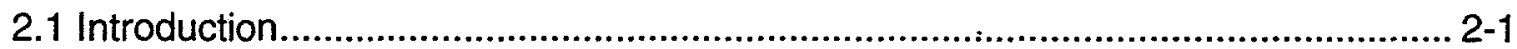

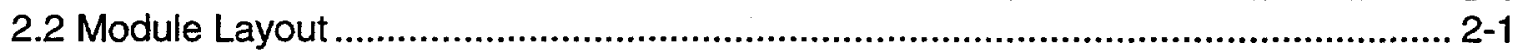

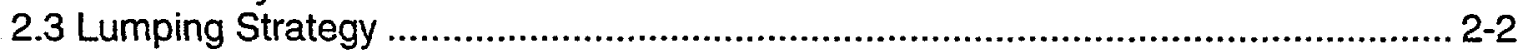

2.4 1-D Lumped TRAC Model......................................................................... 2-2

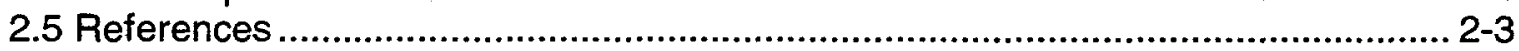

$3 \mathrm{HR}$ and Component Descriptions

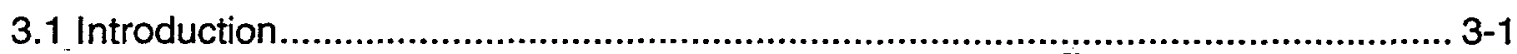

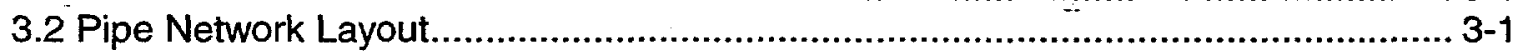

3.3 Heat Exchanger Design .............................................................................. 3-2

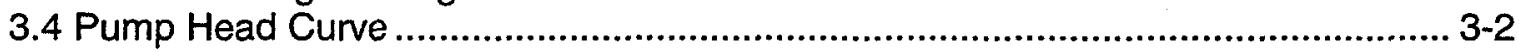

3.5 Steady-State Hydraulic Model....................................................................... 3-3

3.6 Pipe Size Design Study ............................................................................ 3-4

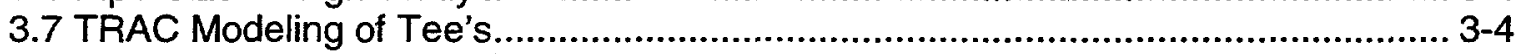

3.8 TRAC Model Steady-State Benchmarking …................................................... 3-6

3.9 TRAC Model Component Layout ......................................................................... 3-6

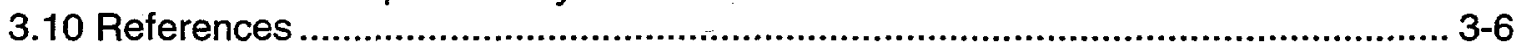

4 Pressurizer Description $\quad$ 4-1

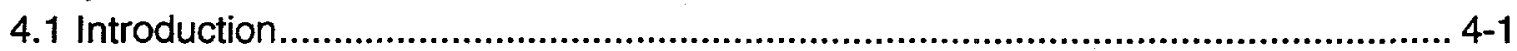

4.2 Pressurizer Design .............................................................................................. 4-1

4.3 Pressurizer Drain Down Design Study ............................................................ 4-1

4.4 TRAC Model .............................................................................................. 4-2

5 RHR and Component Descriptions

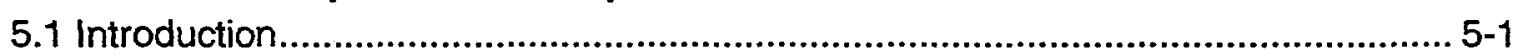

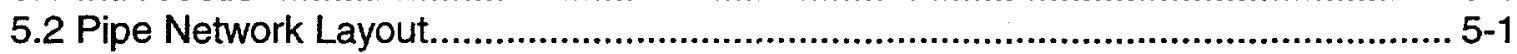

5.3 Heat Exchanger Design ..............................................................................

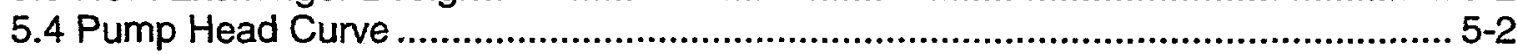

5.5 Steady-State Hydraulic Model............................................................................. 5-2

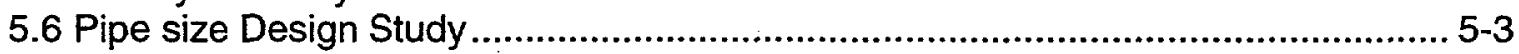

5.7 TRAC Model steady-State Benchmarking ………............................................. 5-4

5.8 TRAC Model Component Layout .................................................................... 5-4

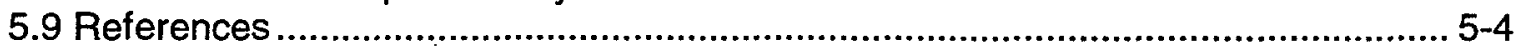


6 Cavity Vessel/Flood System Descriptions

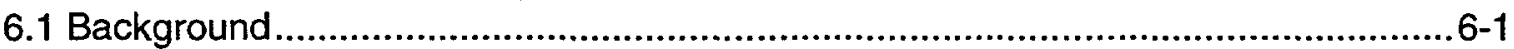

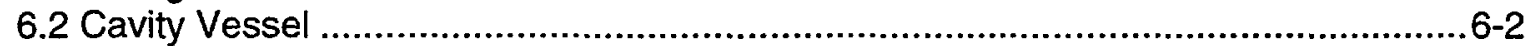

6.2.1 Thermal Coupling of Heat Structures ....................................................... 6-2

6.2.2 Cavity Vessel Vent Valve .......................................................................6-2

6.2.3 Potential Break Locations within Cavity Vessel ..............................................6-2

6.2 Cavity Flood Pool ...............................................................................................6-3

6.3 Cavity Flood Valve and Fill Line .......................................................................6-3

6.4 Simple Vapor-Liquid Equilibrium (VLE) Model...................................................6-3

6.5 References........................................................................................................6-4

7 Secondary HR and RHR Component Descriptions $\quad$ 7-1

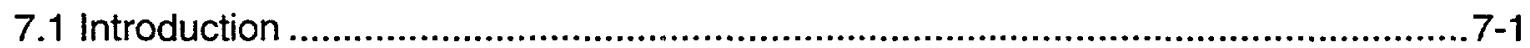

7.2 1-D TRAC Model for HR and RHR Secondary Sides ......................................... 7-1

7.3 References..............................................................................................

8 Control Procedure Using Trip Signals, Signal Variables, and Control Valves

8.1 References.

Appendix A: TRAC Standard Input File for Normal Operation Case

Appendix B: TRAC Graphics Input File for Normal Operation Case

Appendix C: QKTRAC - A Simple Hydraulic Code for HR Assessment

Appendix D: RHRTRC - A Simple Hydraulic Code for RHR Assessment 


\section{List of Figures}

\section{Introduction}

Figure 1-1 Schematic of blanket primary, secondary, and tertiary heat removal systems.

Figure 1-2 Areal view of Target/Blanket building highlighting blanket system layout............................................................................ 1-8

Figure 1-3 Vertical view of Target/Blanket building highlighting blanket system layout.

Figure 1-4 Essential differences between cruciform- and plate-type blanket module components. ..................................................................... 1-10

Figure 1-5 Strategy to establish blanket safety envelope for PSAR purposes.

\section{Module Descriptions}

Figure 2.2-1 Top cross-sectional facemap of 16 blanket system modules. 2-14

Figure 2.2-2

Figure 2.2-3

Front cross sectional view of the blanket system modules. 2-15

Figure 2.2-4

Decoupler and Row 1 cruciform bin geometries.

$2-16$

Figure 2.3-1

Row 2 and Row 3 cruciform bin geometries.

2-17

Figure 2.4-1

Lumping strategy for 1-D TRAC blanket system model.

$2-18$

Figure 2.4-2

1-D TRAC model for Lateral Decoupler / Row 1 Module.

$2-19$

Top cross-sectional facemap of 6 lumped blanket system modules.

Figure 2.4-3

Figure 2.4-4

6 blanket module layout for safety analysis.

Figure 2.4-5

Typical axial power distribution for Row 1 module.

2-22

Typical axial power distribution for Row 2 module.

2-22

\section{$3 \mathrm{HR}$ and Component Descriptions}

Figure 3.2-1 Schematic of the external portion of the blanket primary heat removal system.

Figure 3.2-2 Areal view of the of the external portion of the blanket primary heat removal system. Lengths of straight pipe are in parentheses. ..... 3-9

Figure 3.2-3 Side view of the external portion of the blanket primary heat removal system. Lengths of straight pipe are in brackets.

Figure 3.3-1 Schematic of a blanket primary heat removal system heat exchanger.

Figure 3.4-1

Pump normalized homologous head curves.

Figure 3.5-1

Schematic of the fixed header/pipe connection, showing how the header flow areas are treated to calculate an abrupt contraction form loss coefficient.

Figure 3.7-1

Schematic of dividing Tee pipe components.

Figure 3.7-2

Pressure drop comparison across a symmetric dividing Tee, between a TRAC Tee component and literature loss coefficients.

Figure 3.7-3

Pressure drop comparison across a symmetric dividing Tee, between a TRAC plenum component and literature loss coefficients.

Figure 3.7-4 Pressure drop comparison across a symmetric combining Tee, between a TRAC plenum component and literature loss coefficients. 
Figure 3.7-5 Pressure drop comparison across an asymmetric dividing Tee, between a TRAC plenum component and literature loss

coefficients.

Figure 3.7-6 Pressure drop comparison across an asymmetric combining Tee, between a TRAC plenum component and literature loss coefficients.

Figure 3.9-1 TRAC model component layout of the external portion of the primary HR system.

\section{Pressurizer Description}

Figure 4.2-1 Schematic of the pressurization vessel and the surge line 4-3

Figure 4.3-1

TRAC component layout of a stand-alone drain down model of the pressurizer and surge line. 4-4

Figure 4.3-2 Pressurizer fluid pressures for a simulated internal large-break LOCA located just above the top shield. 4-5

Figure 4.3-3 Pressurizer fluid temperatures for a simulated internal large-break LOCA located just above the top shield. 4-5

Figure 4.3-4

Pressurizer liquid mass flowrates for a simulated internal largebreak LOCA located just above the top shield. 4-6

Figure 4.3-5 Pressurizer void fractions for a simulated internal large-break LOCA located just above the top shield.

Figure 4.3-6 Pressurizer surge line fluid pressures for a simulated internal large-break LOCA located just above the top shield.

Figure 4.3-7 Pressurizer surge line liquid mass flowrates for a simulated internal large-break LOCA located just above the top shield.

Figure 4.3-8 Pressurizer surge line void fractions for a simulated internal largebreak LOCA located just above the top shield.

\section{RHR and Component Descriptions}

Figure 5.2-1 Schematic of the blanket residual heat removal system. $.5-5$

Figure 5.2-2 Areal and side views of the residual heat removal system.

Figure 5.3-1

Schematic of the blanket residual heat removal system heat exchanger.system.

Figure 5.5-1. Schematic of the break location in the primary heat removal system that was used to determine the fixed inlet header pressure boundary condition for the residual heat removal system simple hydraulic model, (RHRTRC).

Figure 5.8-1 TRAC model component layout of the blanket residual heat removal system.

\section{Cavity Vessel/Flood System Descriptions}

Figure 6.2-1 TRAC component layout for the cavity vessel and blanket module heat structures.

Figure 6.2-2 TRAC component layout for the cavity vessel and cavity flood system.

\section{Secondary HR and RHR Component Descriptions}

Figure 7.2-1 Typical coolant temperature profiles for primary and secondary

heat exchanger sides

Figure 7.2-2 1-D TRAC model for the secondary side of primary HR heat

exchanger. 
Figure 7.2-3 Heat exchanger fluid and heat structure nodalization for 1-D TRAC model under counter-current flow pass.

8 Control Procedure Using Trip Signals, Signal Variables, and Control Valves

Figure 8-1 Control procedure for blanket system component hardware actions...

Figure 8-2 S-shape forcing function curve used in the present analysis. $8-5$

-vii- 


\section{List of Tables}

\section{Introduction}

\section{Module Descriptions}

Table 2.2-1 Module types and geometric dimensions for 16 blanket modules. ........2-4

Table 2.2-2 Typical area fractions for various types of blanket module bins. ...........2.5

Table 2.2-3 Deposited powers, liquid flowrates and module coolant pipe sizes for 16 blanket modules....................................................................2-5

Table 2.2-4 Decay power fractions of full power for lead cruciforms of various locations................................................................................2-6

Table 2.4-1 6 lumped blanket module system model used for the present PSAR analysis ............................................................................2-7

Table 2.4-2 Blanket System Component Descriptions in TRAC Model. .....................2-8

Table 2.4-2 Blanket System Component Descriptions in TRAC Model (continued).

Table 2.4-2 Blanket System Component Descriptions in TRAC Model (continued).

Table 2.4-2 Blanket System Component Descriptions in TRAC Model (continued).

Table 2.4-2 Blanket System Component Descriptions in TRAC Model (continued) ....................................................................... $2-12$

Table 2.4-3 Steady State Conditions.........................................................2-12

Table 2.4-4 6 Lumped Blanket Modules from 16 Full Blanket Module System.......2-13

\section{$3 \mathrm{HR}$ and Component Descriptions}

Table 3.6-1 Estimated required and available NPSH for HR piping. 3-7

Table 3.8-1 Comparison between TRAC and simple hydraulic model pressure profile for HR piping loop.

\section{Pressurizer Description}

\section{RHR and Component Descriptions}

Table 5.6-1 Estimated required and available NPSH for RHR piping......................4-4

Table 5.7-1 Comparison between TRAC and simple hydraulic model pressure profile for RHR piping loop.

\section{Cavity Vessel/Flood System Descriptions}

\section{Secondary HR and RHR Component Descriptions}

\section{Control Procedure Using Trip Signals, Signal Variables, and Control Valves}

Table 8-1 Control signal variables used in the APT blanket system model. $8-2$

Table 8-2

Trip signal variables used in the APT blanket system model.

Table 8-3

Design basis accident scenarios for the APT blanket system safety analysis. 


\section{Introduction}

This report documents the approaches taken in establishing a 1-dimensional integrated blanket system model using the TRAC code, developed by Los Alamos National Laboratory. Descriptions of all major components are provided in this report. Where appropriate, discussions include the processes used and justifications made in making key design decisions. In certain circumstances supporting analyses were performed to support or verify the modeling approach employed. Due to the lack of key design information on many of the blanket system components within the Conceptual Design Report [1], many of the components presented and modeled within this report represent the author's best judgment. However, when design specifications were made available (either within the CDR [1] or through correspondence with LANL personnel), they were incorporated into the system model. Reference [8] discusses the blanket system safety analysis methodology pertaining to the efforts documented within this report.

\subsection{Overview of Blanket System}

Figure 1.1-1 provides a schematic highlighting the various cooling systems associated with the overall blanket system. The blanket system consists of one $100 \%$ capacity primary heat removal (HR) coolant loop with light water as the working fluid. Two $100 \%$ primary residual heat removal (RHR) coolant loops are also available (upon actuation) with light water as their working fluid as well.

The HR and RHR coolant loops are connected to common fixed inlet/outlet headers that reside within a cavity vessel. System pressure is controlled using a large pressurizer vessel that is directly connected to the inlet fixed header through a 10 inch schedule 40 surge line. Multiple (16) heat generating lead/aluminum module units are also connected to these fixed headers and reside within the cavity vessel proper. The cavity vessel represents one level of confinement, while a second level of confinement is established within the Target/Blanket facility using a ventilation system exhausting through HEPA filters and the stacks.

As an ultimate heat sink for certain classes of accidents, a cavity flood system has been provided. A cavity flood pool (upon actuation) dumps by gravity sufficient light water into the cavity vessel space to cover the blanket components and fixed headers. Secondary and tertiary cooling systems exist for the HR, RHR, and cavity vessel as shown in Fig. 1.1-1.

Based on available design guidance and Target/Blanket building geometric constraints, blanket component and piping layouts for the HR, RHR, and pressurizer primary coolant systems were established. Figures 1.1-2 and 1.1-3 provide areal and vertical views of the blanket systems, respectively. In Fig. 1.1-2 note that only the lower two HR primary coolant pumps are in the system model (the upper third pump is in a standby mode and is valved out-of-service during normal operation (NO) and under accident conditions). Figures 1.1-2 and 1.1-3 are approximately to scale with respect to component sizes and locations.

At the bin level within a given blanket module, the original design was of a cruciformtype concept as shown in the upper portion of Fig. 1.1-4. This design consisted of discrete heat structures made of aluminum cladded lead cruciforms placed within a rectangular aluminum structure defined as a local bin. Several bins ganged together 
constitute an individual blanket module. Within each bin, neighboring cruciform components were spaced apart using discontinuous longitudinal ribs. Within these bins was a continuous coolant channel with many sub-channels of varying widths due to fabrication tolerances. This cruciform-type design was based on preliminary NO design calculations only. Later a preliminary safety assessment for the cruciform design was performed [2,3]. The NO calculations in Ref. [2] confirmed the earlier design analyses; however, design concerns emerged for various accident scenarios considered [3]. Reference [3] prompted a need for a new design improvement study. Recognizing (1) that beam shutdown occurs very rapidly, (2) that a significant reduction in decay power occurs, and (3) these lead components are massive and contain large thermal inertia, the possibility existed to redesign the blanket modules at the bin level. The design improvement study centered around:

- taking advantage of the low decay heat conditions (i.e., use heat conduction as a major means for heat removal from the bins);

- eliminating the difficult analysis requirements associated with complex subchanneling due to varying rib geometries.

The expectations for the outcome of this design improvement were more robust blanket modules with much simpler safety analysis requirements, both experimentally and numerically. These expectations were met based on a plate-type design as shown in the lower portion of Fig. 1.1-4. The main features of the plate-type design is to have a continuous heat structure at the bin level with discrete one-dimensional flow channels dispersed throughout the heat structure. Safety analyses (both NO and various bounding accident scenarios) were performed to better quantify the thermal/hydraulic benefits over the earlier cruciform design [4].

\subsection{Modeling Strategy}

The thermal/hydraulic modeling aspects for the Target/Blanket Systems have been broken into several basic modeling components. Due to the natural separation (i.e., separated flow loops) that occurs between the Target System, the Blanket System, and the Cavity System, these systems are in most cases modeled separately. However, under certain accident conditions (e.g., flooded cavity conditions) these systems can interact materially and thermally. In these situations these system models may have one or two way communication between each other through appropriate boundary conditions. Such de-coupling of interacting systems necessitates the need for a limited amount of iterations to be performed or some set of bounding boundary conditions be applied.

The detailed modeling of every module and its individual sub-components within the blanket system is unnecessary and perhaps not currently achievable. Due to the complexity of flow paths and geometric shapes of heat structures, the Blanket Primary Coolant System (BPCS) has been subdivided into two basic analysis models:

- First, an integrated 1-D system model was chosen that contains all major components, systems, and heat structures deemed necessary, and making use of varying and appropriate degrees of lumping wherever possible. The integrated system model is based on the TRAC code [5]. The TRAC code was modified to incorporate grahpics output file generation [7]. 
- Second, the integrated 1-D system model will provide local boundary conditions to a more detailed multi-dimensional bin model at the component level within a given "hot" module. The detailed bin model is based on a modified version of the FLOWTRAN-TF code [6].

As indicated in Fig. 1.2-1, the overall safety strategy taken relies on a separated approach (i.e., a system model supplying boundary conditions to a more detailed bin model). The two major advantages of this type of approach are: (1) the ability to investigate certain aspects of the facility in much greater detail and (2) the ability to perform a significantly larger number of sensitivity analyses on individual components. The disadvantages are: (1) the strategy used and adequacy of such lumping techniques must be formulated and demonstrated and (2) a limited number of iterative analyses between model components must be performed. With regard to the TRAC system model, Fig. 1.2-2 outlines the solution strategy approach taken.

\subsection{Analysis Requirements of Integrated System Model}

\subsubsection{System Model Requirements}

With the integrated blanket system model, overall system behavior for a variety of different thermal/hydraulic $(T / H)$ states can be considered. Briefly these states include: (1) a flow regime comprised of single phase subcooled flowing liquid, (2) a slightly-tohighly subcooled nucleate boiling regime, (3) a dominantly bulk boiling regime, and (4) a post-CHF transition boiling regime. A broad range of accident scenarios can be addressed with the system model. The following is a partial list of key accident scenarios associated with the blanket system that may need to be considered for analysis in establishing an overall safety envelope:

- Internal and external to the cavity vessel large break (LB) loss-of-coolant accidents (LOCA's) within a primary HR loop, RHR loop, or pressurizer surge line. A single guillotine double-ended pipe break (DEGB) at varying locations may be considered.

- Internal and external to the cavity vessel small break (SB) LOCA's within the primary loops. A single break with varying size and location may be considered.

- Loss-of-flow accident (LOFA) for the primary coolant flow. The loss of a single and both primary pumps may be considered.

- Loss of secondary side heat sink accident (LOHS). The loss of secondary coolant flow of a single and both second sides may be considered.

- Loss of Off-Site power and Station Blackout initiating events may be considered.

- Local flow blockages within the primary coolant loops or blanket modules. Varying degrees of blockage and location may be considered (at the module inlet level and among the discrete flow channels within module bin).

- A local power density insertion accident resulting from beam expander magnet failure (a beam focusing accident).

In order to accommodate the various key features of the above potential list of accident scenarios, the blanket system model must include both branches of the HR coolant loops associated with its two online pumps, as well as one of the $100 \%$ capacity residual heat removal (RHR) systems. To maintain reasonable computing run times and overall memory requirements, individual discrete flow channels within a module bin, as well as 
individual bins that make up a complete module unit, it may be necessary not to modeled them separately. Instead, the blanket system model may required a limited number of lumped modules that also contain a limited number of composite (and lumped) heat structures. The level of adequacy in the lumped modules and composite heat structures employed in a blanket system model can be assessed by: (1) comparison to separate analytical models, (2) comparison to the more detailed bin model, or (3) comparison to standalone sub-models of the system. The blanket system model provides the key boundary conditions necessary for performing the more detailed thermal analyses at the bin level.

\subsubsection{System Code Selection}

Selection of a system code capable of accommodating the various stated requirements/objectives above was necessary. At the outset it was recognized that to be able to handle the potentially diverse thermal/hydraulic $(T / H)$ conditions that may be present during a given accident sequence, a flexible two-fluid $\mathrm{T} / \mathrm{H}$ based algorithm is necessary. Some of the more basic features and options needed are:

- A two-fluid formulation whose constitutive packages can reasonably handle the needs of a APT blanket modules and their expected T/H conditions.

- Capable of performing transient single \& two-phase flow within 1-D system components and piping branches (i.e., models the axial motion of liquid and vapor phases).

- A lumping strategy to reduce discrete flow channels and heat structures down to a single effective one dimensional rod and channel.

- Lumping together of multiple flowpaths within modules and potentially among modules.

- For natural circulation conditions secondary light water cooling systems coupled to their primary sides through heat exchangers. Ability to reduce secondary side modeling down by handling them as BCs within a heat exchanger component.

- A pressurizer explicitly connected to the system model.

- Handle inlet and outlet headers are modeled as common manifolds.

- Tee components to model pipe branching.

- Centrifugal pump models for the HR and RHR loops based on appropriate pump head curves for single and two-phase flow conditions. Pump coastdown characteristics are included to handle flywheel inertia.

- For internal cavity break locations a special cavity model coupled to the HR and RHR systems.

The currently available series of TRAC codes at LANL meet most of the requirements/objectives stated above. During model development some level of development/upgrade to the TRAC code may be required with regard to its constitutive packages. Various other available candidates (e.g. RELAP5, RETRAN, etc.) are capable of handling the transient, multi-phase flow, and conjugate heat transfer behavior expected; however, the TRAC code was selected for the system model for consistency since target and cavity flood systems are already TRAC based models. 


\subsection{System Design Versus Model}

Due to the initial lack of firm designs and continued design iterations on many of the major blanket components, several of the components described in this report do not correspond to the information provided in the Conceptual Design Report [1]. As such, it is anticipated that many of the systems and components described in the following sections will be significantly altered when the next set of blanket safety analyses are performed. The following two major design modifications will most likely be incorporated in future safety analyses:

- Lumped cruciform-type module components are being used in the current system model. Based on an earlier assessment analysis [2,3] and then a follow up design improvement study [4], plate-type module components were chosen in early June, 1997. A complete set of plate-type component designs originally due in August, 1997 , are still unavailable as of this writing.

- Piping layout of the HR and RHR primary loops are to be modified to better handle large break LOCA's internal to the cavity. The current layout contains inverted $P$. traps (see Fig. 1.1-3) that result in flashing and collection of steam in their high points.

The current methodology consists of two (varying in complexity) system models that provide local boundary conditions to a more detailed bin model at the component level within a given "hot" module. The methodology for the overall system model(s) is to separate accident analyses into two separate categories:

- Design basis accident scenarios (e.g., normal operation, anticipated operational occurrences, loss-of-flow accidents, and internal/external large break loss-of-coolant accidents).

- Accident scenarios of a bounding nature (e.g., the internally dry module with a flooded cavity space, beyond design basis accidents, residual risk events).

Each category places different computational demands on the analysis tools and available computer resources. For category 1 the plan is to use the lumped six module TRAC system model where justification for the additional module lumping is provided using our most detailed 16 module model under similar steady-state conditions. For category 2 we will most likely have to abandon TRAC at some point in the scenario and make bounding assumptions or separate evaluation modeling calculations.

The current one-dimensional blanket system models (both 16 and 6 module cases) have been developed using version 5.4.28a of the TRAC-P code [5]. The 1-D detailed blanket system model consists of 16 unique blanket modules based on the existing cruciform-type design components (the necessary design specifications required to develop a plate-type set of modules is currently not available but should be forthcoming in late February). During the assessment period for the detailed blanket system model, it was recognized that lumping of various similar modules could be performed without adversely affecting the results from transient accident analyses. It was also recognized that: (1) the helium components could be deleted; and (2) a reduction in heat exchanger nodalization was acceptable. A lumping strategy was developed based on module similarity, deposited power levels, and locations that resulted in a total of six separate lumped modules and reduced computational effort requirements. The six lumped modules are, respectively: the first-row lateral modules; the second-row lateral modules; the decoupler/row 1 downstream module; the row 1/row 2 downstream module; the row 
2/row 2 downstream module; and the remaining low-power modules (that consists of the upstream module, the four bottom modules, and the two top modules). For providing blanket system safety analysis in support of the initial PSAR, this report will focus primarily on the six module model. Only limited assessments are provided comparing the detailed 16 module model to the lumped six module model.

\subsection{Special Purpose Standalone Models}

Special purpose thermal/hydraulic models have also been developed to assist in verification of various components of the integrated system model. For example, separate steady-state hydraulic models of the primary heat removal and residual heat removal systems were developed (i.e., the piping and components from the fixed inlet header to the fixed outlet header). These models were used to assess the single phase steady-state hydraulic behavior of the appropriate sections of the TRAC system model when compared to standard textbook hydraulic models. Also, a standalone model of the cavity flood systems based on a simple thermodynamic vapor-liquid-equilibrium (VLE) model was created. This VLE model provides insights into the timing of key events during the flooding phase.

\subsection{References}

1. "APT Conceptual Design Report," Los Alamos National Laboratory report, LA-UR97-1329 (April 1997).

2. L. L. Hamm and M. A. Shadday, "APT Blanket Safety Analysis: Preliminary Hot Subchannel Model Results," Westinghouse Savannah River Company, Section memorandum number SRT-EMS-97-0024, April 9, 1997.

3. L. L. Hamm and M. A. Shadday, "APT Blanket Safety Analysis: Preliminary Internally Dry Flooded Cavity Results," Westinghouse Savannah River Company, Section memorandum number SRT-EMS-97-0030, May 2, 1997.

4. L. L. Hamm and M. A. Shadday, "APT Blanket Safety Analysis: Preliminary Thermal Conduction Analysis of the B\&W Plate-Type Blanket Assembly (U)," Westinghouse Savannah River Company, Section memorandum SRT-EMS-970039, June 2, 1997.

5. Safety Code Development Group, "TRAC-PF1/MOD2: An Advanced Best Estimate Computer Program for Pressurized Water Reactor Thermal-Hydraulic Analysis," Los Alamos National Laboratory report LA-12031-M, Vol. 1 (NUREG/CR-5673), (July 21, 1993).

6. L. L. Hamm, S. Y. Lee, M. A. Shadday, and F. G. Smith, "FLOWTRAN-TF Code Modifications made for APT Blanket Safety Analyses," Westinghouse Savannah River Company, WSRC-TR-98-0056 (July 1998).

7. L. L. Hamm, S. Y. Lee, M. A. Shadday, and F. G. Smith, III, "TRAC Code Modifications made for APT Blanket Safety Analysis," Westinghouse Savannah River Company, WSRC-TR-0054 (July 1998).

8. L. L. Hamm, S. Y. Lee, M. A. Shadday, and F. G. Smith, III, "APT Blanket Safety Analysis Methodology," Westinghouse Savannah River Company, WSRC-TR0052 (May 1998). 


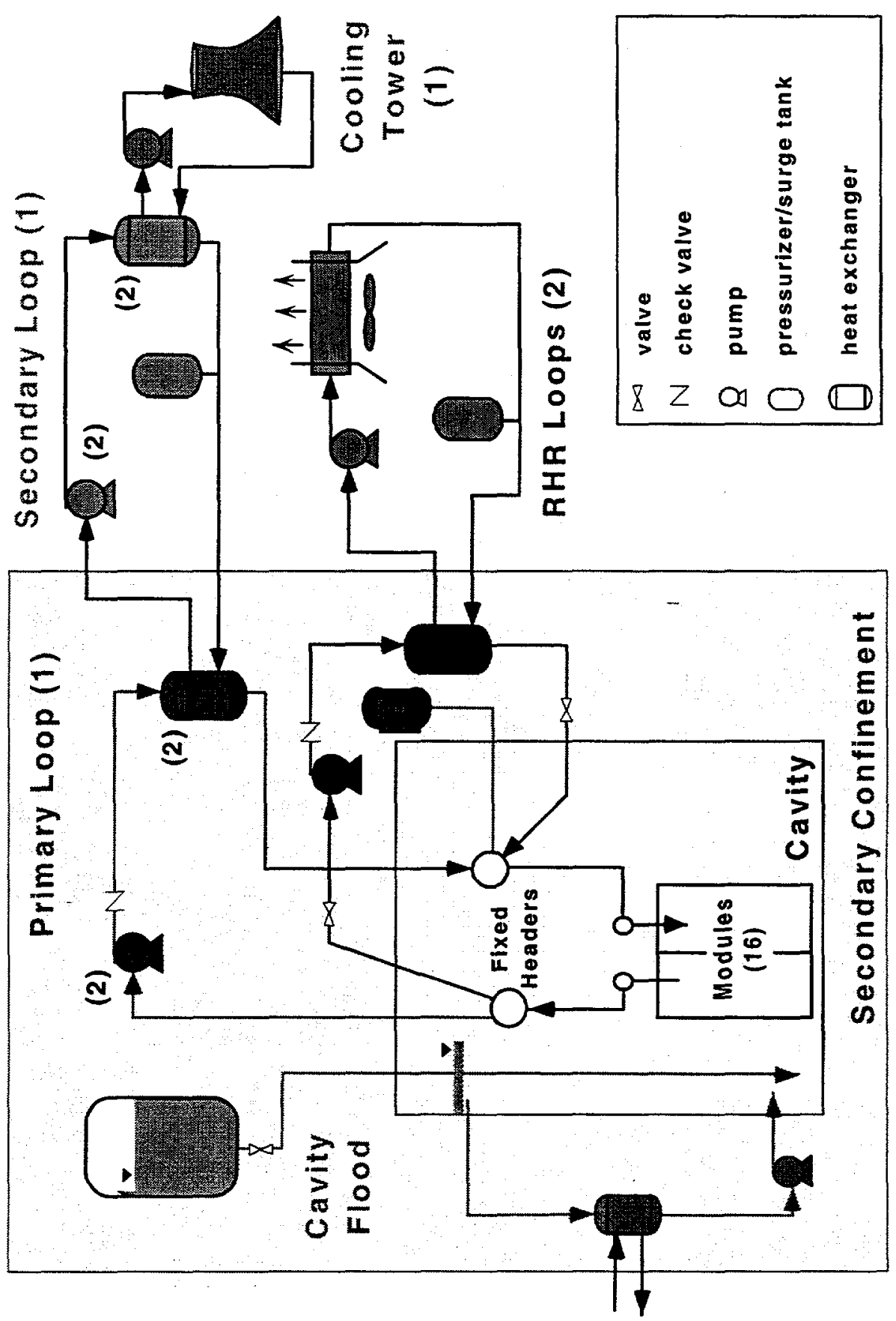

Figure 1.1-1 Schematic of blanket primary, secondary, and tertiary heat removal systems. 


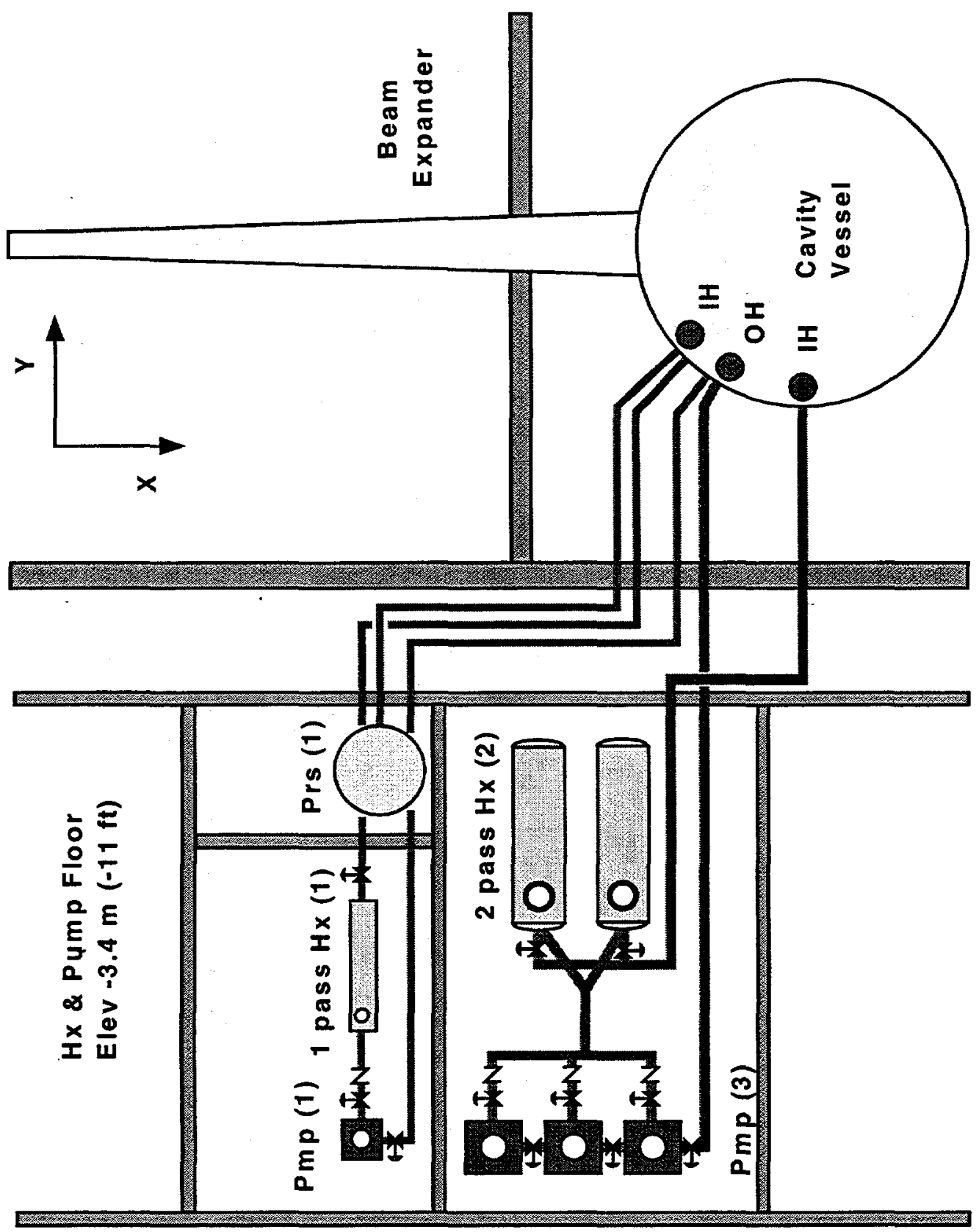

Figure 1.1-2 Areal view of Target/Blanket building highlighting blanket system layout. 


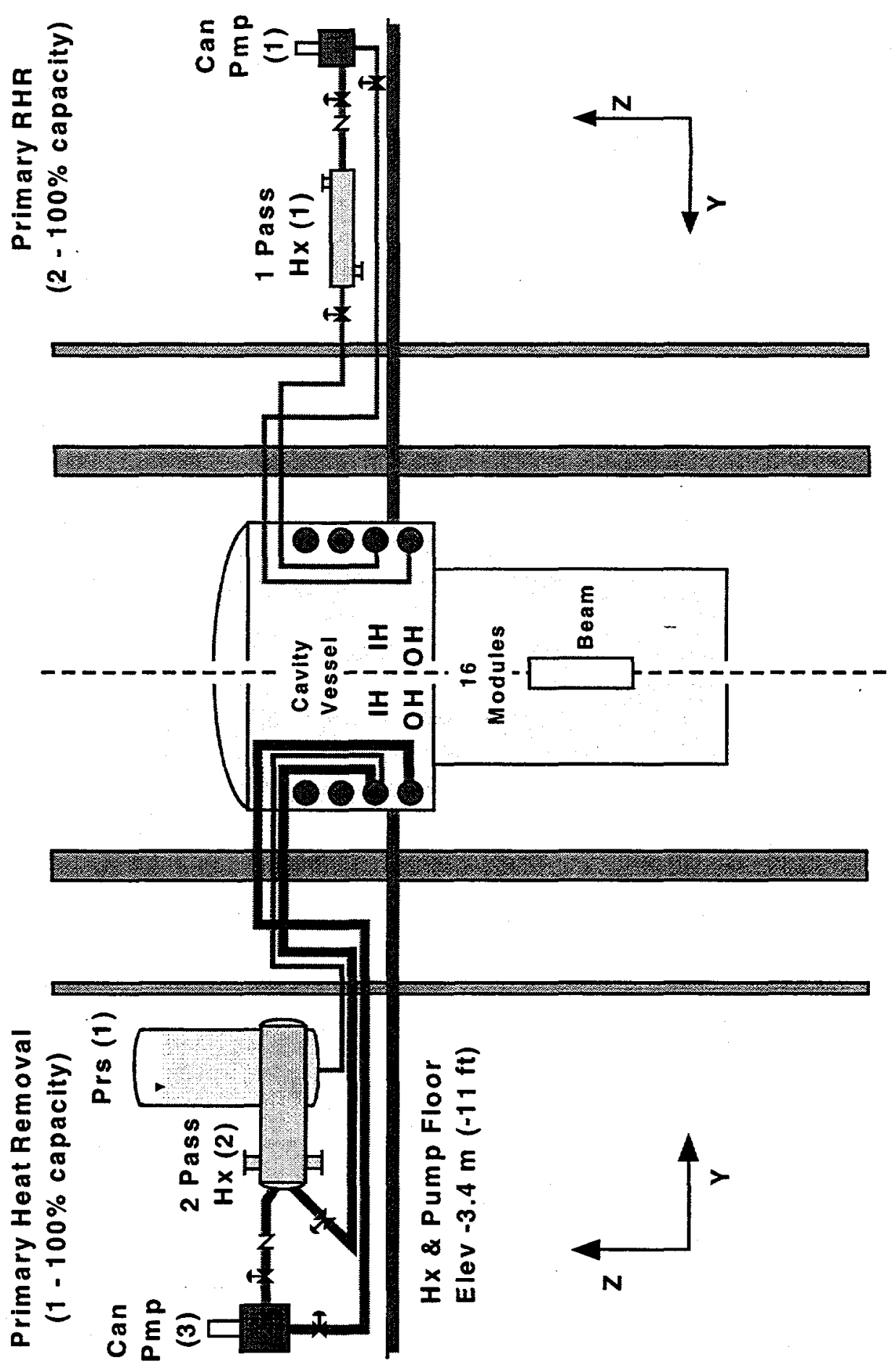

Figure 1.1-3 Vertical view of Target/Blanket building highlighting blanket system layout. 


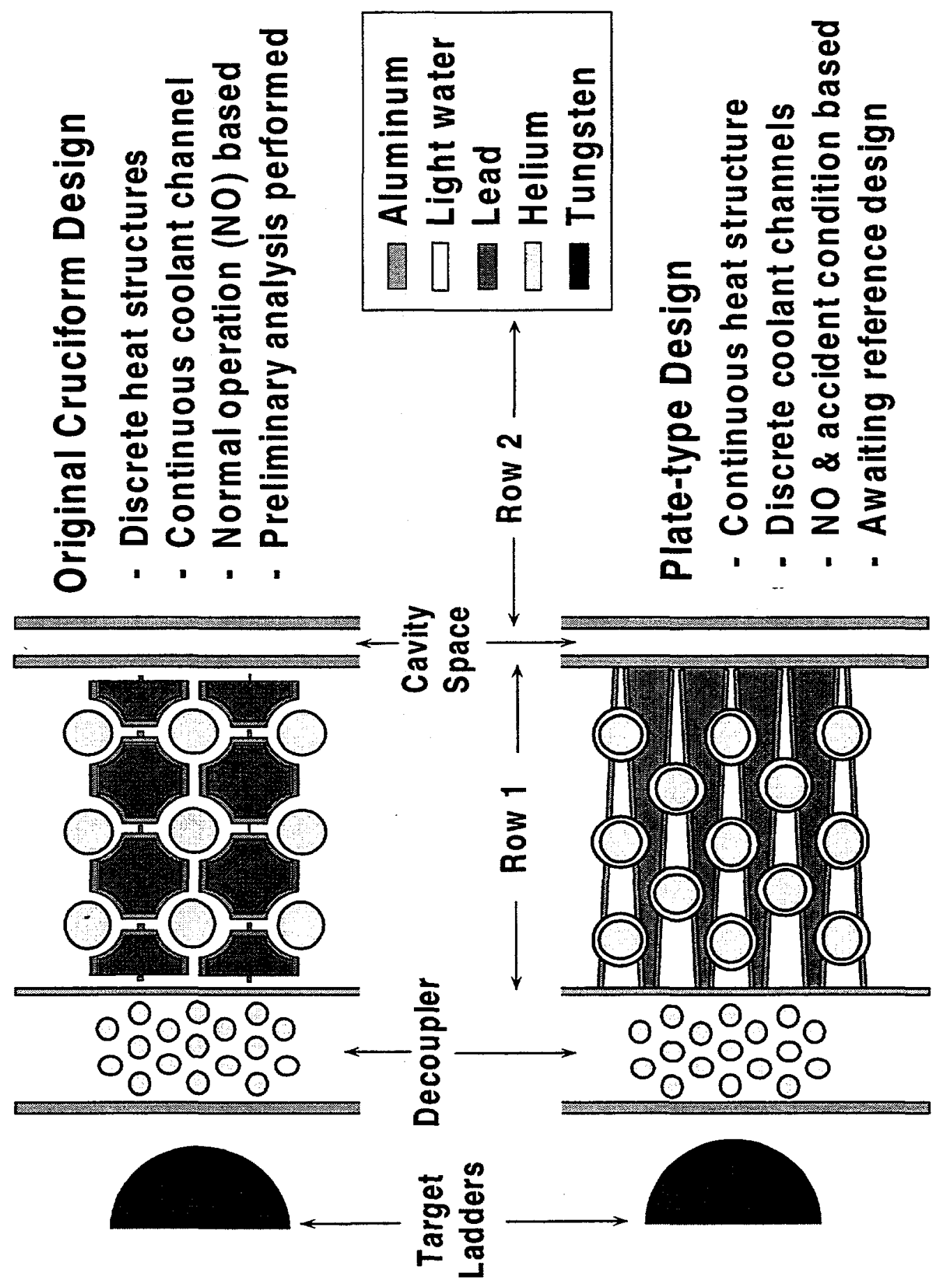

Figure 1.1-4 Essential differences between cruciform- and plate-type blanket module components. 


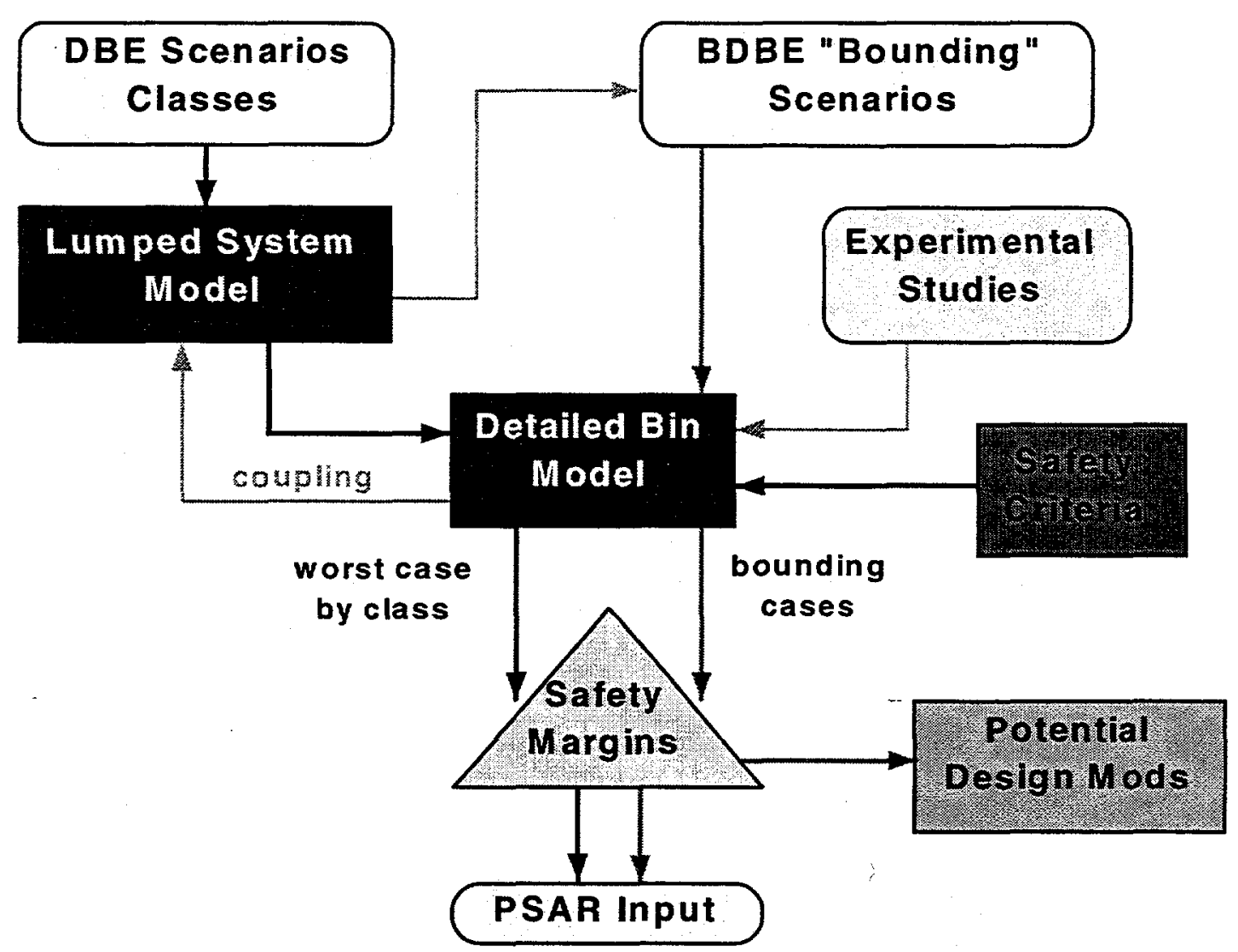

Figure 1.2-1 Strategy to establish blanket safety envelope for PSAR purposes. 


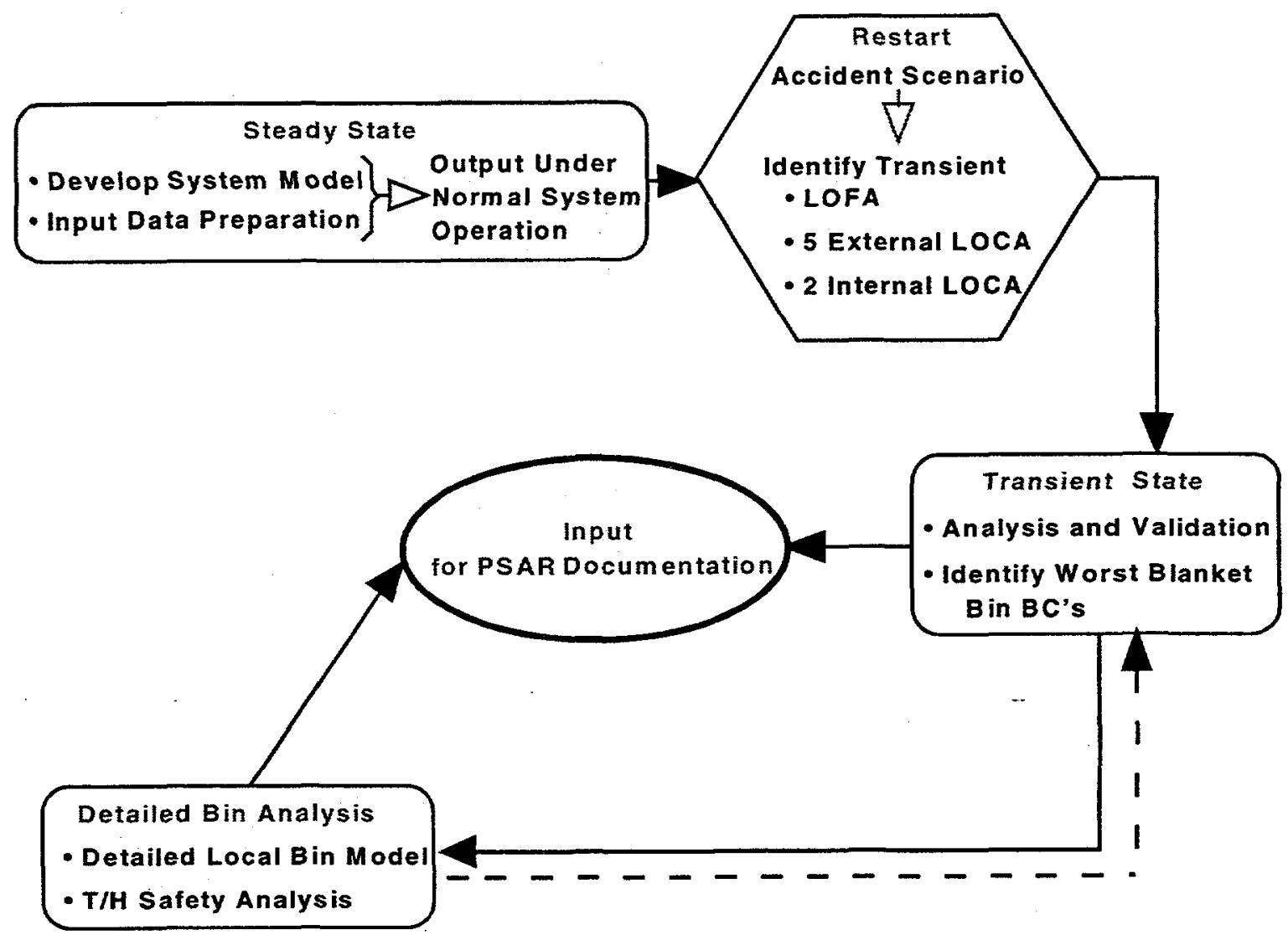

Figure 1.2-2 Solution approach using 1-D TRAC model. 


\section{Module Descriptions}

\subsection{Introduction}

The blanket system is designed as a modular-type assembly to allow for replacement and maintenance of short-lived components. Tritium will be produced with the source neutrons generated as a result of spallation from primary high energy protons striking tungsten targets. The main function of the blanket system is to enhance neutron production and to catch high energy particles scattered out of the tungsten neutron source. From the release of neutrons and the interaction of the high energy particles within the blanket materials; approximately 57 MW of thermal energy is deposited within the blanket region under normal operating conditions. This corresponds to about $48 \%$ of the total thermal energy deposited in the APT target/blanket system [1], and is an important input parameter used in the thermal-hydraulic design and safety analysis.

\subsection{Module Layout}

The blanket system consists of 18 different blanket modules, surrounding the central target system. For each of the front and back lateral modules located next to the target region, two lateral modules are merged into a single pipe through a T-junction and connected to fixed headers, so 16 separate thermal-hydraulic loops are connected to two fixed headers for all the 18 separate blanket modules [2]. Figure 2.2-1 shows the entire blanket face map with 16 individual modules consisting of: six lateral modules, three downstream modules, one upstream module, two top modules and four bottom modules. Each blanket module is subdivided into two different regions, either a decoupler and lead cruciform row or two different cruciform rows, depending on the type of blanket module. One region is for downflow, and the other is for upflow. There is no flow communication between regions within a blanket module. Each of the two regions contains a number of rectangular bins, and each bin has a number of aluminum tubes and blanket rows. Figure 2.2-2 shows that each blanket module has two flow zones and that the decoupler is located adjacent to the target region, oriented vertically. The lateral decoupler and row 1 blanket are $2.8 \mathrm{~m}$ high and extend to just below the bottom of the tungsten target ladder. The other vertical blanket modules are all $3.5 \mathrm{~m}$ high. Table 2.2-1 lists blanket types, geometric dimensions, and number of bins for each module. Figure 2.2-3 shows bin cruciform geometries for the decoupler / Row 1 module. The decoupler surrounds the target ladder region and consists of three or four layers of tightly packed aluminum tubes containing helium. Light water coolant flows downward outside the tubes under moderate pressure. The helium in this region mainly absorbs low energy neutrons that scatter from the lead cruciform rows toward the target region, thus maximizing neutron utilization and minimizing neutron absorption in the target tungsten. Four or five layers of closely-packed lead cruciforms surround the decoupler region. The present blanket system has five different cruciform designs in Row 1 through Row 5, from the central target region to the blanket system boundary. Moving outward radially from the target ladder, the lead power density drops rapidly. To accommodate this variation, the amount of lead, $\mathrm{He}$, aluminum, and coolant water is tailored to the deposited power density to optimize the tritium production and thermal-hydraulic safety. Table 2.2-2 shows typical material compositions for the three blanket rows considered in this analysis. The power densities for each blanket module require minimum liquid flow rates for each module, to satisfy the thermal-hydraulic design criteria. Table 2.2-3 shows deposited powers, liquid flowrates, and connection pipe sizes for all of the 16 blanket modules. Figure 2.2-4 shows geometric dimensions for Row 2 and Row 3 bins. 
There are two separate light water heat removal systems to remove the deposited heat from the blanket modules. One is for high and medium power blanket modules (Row 1, Row 2, and Row 3 ), and the other is for low power blanket modules (Row 4 and Row 5, The reflector, and shielding regions). The Row 4 and Row 5 modules are not included in the present blanket safety analysis since they have a separate coolant system and they are very low power modules. Figure 2.2-2 shows the vertical layout of the blanket modules. Table 2.2-4 presents transient decay power fractions of full power for the various types of lead cruciforms used in the modular blanket system.

\subsection{Lumping Strategy}

Within a module blanket bin, there are coolant passages between the lead cruciforms and bin boundaries, between neighboring cruciforms, and between the cruciforms and He tubes. These flow passages provide adequate cooling for the blanket module while minimizing the pressure drop through the blanket assembly. A single flow channel around a lead cruciform is formed by four neighboring aluminum tubes, as shown in Fig. 2.2-3 and Fig. 2.2-4. There is interaction between the adjacent flow channels due to cross-flow and turbulent flow mixing within a module bin. Before a local hot channel in a module can be analyzed, the average flow conditions of the module assembly must be known, to provide local boundary conditions for a detailed hot subchannel model. The module subchannels are lumped into a single annular flow channel that is part of a comprehensive heat removal system model. This flow channel is hydraulically similar to a single closed subchannel, since there is a repetitive subchannel pattern within a bin. The thermal and hydraulic lumped approach evaluates the enthalpy rise in the flow channel by using the subchannel analysis. The annular channel area is determined by preserving area fractions for each material of the equivalent subchannel, as provided in Table 2.2-3. Figure 2.3-1 shows a one-dimensional thermal-hydraulic conjugate model for blanket module 1 , used with version 5.4.28a of the TRAC-PF1/MOD2 code.

\subsection{1-D Lumped TRAC Model}

The one-dimensional detailed blanket system model consists of 16 unique blanket modules based on the existing cruciform-type design (the necessary design specifications required to develop a plate-type set of modules are currently not available, but should be forthcoming in late February). Each module is separately connected to the two fixed coolant headers, as shown in Fig. 2.4-1. Based on the present CDR information, there are 16 pipe connections between the plenum of each module and the coolant fixed headers. The model contains about 400 system components, including the primary coolant loop and the residual heat removal (RHR) systems, along with component control signals for transient loss-of-coolant accident (LOCA) simulations. The detailed geometric dimensions and deposited power densities for each blanket module are based on existing design information. Table 2.2-2 shows deposited thermal power, coolant flowrate, and connection pipe size for each of the 16 modules.

During the assessment period of the detailed blanket system model, it was recognized that lumping of similar modules could be performed without adversely affecting the results of transient accident analyses. It was also recognized that the helium components could be deleted and a reduction in the heat exchanger nodalization is acceptable. A lumping strategy. was developed based on module similarity and deposited power levels that resulted in a total of six separate lumped modules and reduced computational effort requirements. The six lumped modules are, respectively: the first-row lateral modules; the second-row lateral modules; the decoupler / Row 1 downstream module; the Row 1 / Row 2 downstream module; the Row 2 / Row 2 downstream module; and the remaining low-power modules, which consist of the 
TRAC BLANKET SYSTEM MODEL.

upstream module, the four bottom modules, and the two top modules. Figure 2.4-2 shows the cross-sectional face map for the 6 module blanket system. Table 2.4-1 summarizes the lumped module description, thermal deposited power, and connection pipe sizes of each of the 6 blanket modules, as modeled in the present one-dimensional lumped approach. Different numbers of mesh cells are used for the $1^{\text {st }}$ lateral and $2^{\text {nd }}$ lateral modules since they have different modular heights, as shown in Table 2.2-1. For example, 5 fluid cells were used for the $1^{\text {st }}$ lateral module, and 7 fluid cells were used for $2^{\text {nd }}$ lateral module. 6 radial cells and 5 or 7 axial node cells in the heater structure region of each subchannel were used to compute metal temperatures. Table 2.4-2 lists the component ID number, number of cells, and a brief model description for each of the blanket system components, including the external primary coolant loop system and the residual heat removal system, which were included in the one-dimensional 6 module system model. Figure 2.4-3 shows all the module components and connection pipes within the cavity vessel, as modeled in the present 6 blanket module model. Circles in Fig. 2.4-2 represent PLENUM components, and rectangles stand for PIPE or HEAT STRUCTURE components. PLENUM components $80,81,83,85,87$, and 89 are potential pipe break points for internal LOCA simulations. Figure 2.4-4 and Fig. 2.4-5 show typical power profiles, in the flow direction, for Row 1 and Row 2.

Table 2.4-2 summarizes the model results of key thermal-hydraulic parameters under normal operation conditions. Benchmarking was performed at steady-state nominal conditions to verify the results of the lumped six module model with the earlier results obtained from the 16 module model. Table 2.4-3 shows the benchmarking results.

\subsection{References}

1. "APT Conceptual Design Report," Los Alamos National Laboratory report, LA-UR-97-1329 (April 1997).

2. R. Kapernick, "Thermal-Hydraulic Design of APT Blanket", Presented at Thermal-Hydraulic Group Meeting, Los Alamos National Laboratory, May 14, 1997. 
TRAC BLANKET SYSTEM MODEL

Table 2.2-1 Module types and geometric dimensions for 16 blanket modules.

\begin{tabular}{|c|c|c|c|c|}
\hline $\begin{array}{l}\text { 16 Module } \\
\text { Number } \\
\text { (Location) }\end{array}$ & $\begin{array}{l}\text { Module Types: } \\
\text { downtlow/ upilow: }\end{array}$ & $\begin{array}{l}\text { Hudraulic } \\
\text { Diameter } \\
\text { downilow / upflow }\end{array}$ & $\begin{array}{l}\text { No of Bins } \\
\text { downilow? } \\
\text { uptlow }\end{array}$ & $\begin{array}{l}\text { Module } \\
\text { length } \\
(\mathrm{m})\end{array}$ \\
\hline $\begin{array}{c}\text { Module } 1 \\
\text { (Front } 1^{\text {st }} \text { Lateral) }\end{array}$ & Decoupler/Row 1 & $\begin{array}{c}8.6117 e-03 / \\
5.0627 e-03\end{array}$ & $8 / 8$ & 2.758 \\
\hline $\begin{array}{c}\text { Module } 2 \\
\text { (Front } 2^{\text {nd }} \text { Lateral) }\end{array}$ & Row 3 / Row 2 & $\begin{array}{c}3.1084 \mathrm{e}-03 / \\
3.1838 \mathrm{e}-03\end{array}$ & $11 / 11$ & 3.500 \\
\hline $\begin{array}{c}\text { Module } 3 \\
\text { (Front } 2^{\text {nd }} \text { Lateral) }\end{array}$ & Row 3/Row 2 & $\begin{array}{c}3.1084 \mathrm{e}-03 / \\
3.1838 \mathrm{e}-03\end{array}$ & $11 / 11$ & 3.500 \\
\hline $\begin{array}{c}\text { Module } 4 \\
\text { (Back } 1^{\text {st }} \text { Lateral) }\end{array}$ & Decoupler/Row 1 & $\begin{array}{c}8.6117 e-03 / \\
5.0627 e-03\end{array}$ & $9 / 9$ & 2.758 \\
\hline $\begin{array}{c}\text { Module } 5 \\
\text { (Back } 2^{\text {nd }} \text { Lateral) }\end{array}$ & Row 3 / Row 2 & $\begin{array}{c}.1084 \mathrm{e}-03 / \\
3.1838 \mathrm{e}-03 \\
\end{array}$ & $11 / 11$ & 3.500 \\
\hline $\begin{array}{c}\text { Module } 6 \\
\text { (Back } 2^{\text {nd }} \text { Lateral) }\end{array}$ & Row 3 / Row 2 & $\begin{array}{c}3.1084 \mathrm{e}-03 / \\
3.1838 \mathrm{e}-03\end{array}$ & $11 / 11$ & 3.500 \\
\hline $\begin{array}{c}\text { Module } 7 \\
\left(1^{\text {st }} \text { Downstream }\right)\end{array}$ & Decoupler / Row 1 & $\begin{array}{c}9.9388 \mathrm{e}-03 / \\
5.7015 \mathrm{e}-03\end{array}$ & $4 / 4$ & 2.758 \\
\hline $\begin{array}{c}\text { Module } 8 \\
\left(2^{\text {nd }} \text { Downstream) }\right.\end{array}$ & Row 2 / Row 1 & $\begin{array}{c}3.1198 \mathrm{e}-03 / \\
5.1537 \mathrm{e}-03\end{array}$ & $8 / 8$ & 3.500 \\
\hline $\begin{array}{c}\text { Module } 9 \\
\left(3^{\text {rd }} \text { Downstream }\right)\end{array}$ & Row 2 / Row 2 & $\begin{array}{c}3.1519 \mathrm{e}-03 / \\
3.1519 \mathrm{e}-03\end{array}$ & $12 / 12$ & 3.500 \\
\hline $\begin{array}{l}\text { Module } 10 \\
\text { (Blanket } \\
\text { Upstream) }\end{array}$ & Decoupler/Row 2 & $\begin{array}{l}1.0594 \mathrm{e}-02 / \\
2.8707 \mathrm{e}-03\end{array}$ & $\begin{array}{c}\text { horizontal flow } \\
22 / 50\end{array}$ & $1.020 / 1.077$ \\
\hline $\begin{array}{c}\text { Module } 11 \\
\text { (Lower Front } \\
\text { Upper) }\end{array}$ & Decoupler / Row 2 & $\begin{array}{c}9.5264 \mathrm{e}-03 / \\
3.6243 \mathrm{e}-03\end{array}$ & $\begin{array}{c}\text { horizontal flow } \\
5 / 5\end{array}$ & 2.114 \\
\hline $\begin{array}{c}\text { Module } 12 \\
\text { (Lower Front } \\
\text { Lower) }\end{array}$ & Row 2 / Row 2 & $\begin{array}{c}3.6243 e-03 / \\
3.6243 e-03\end{array}$ & $\begin{array}{c}\text { horizontal flow } \\
5 / 5\end{array}$ & 2.114 \\
\hline $\begin{array}{c}\text { Module } 13 \\
\text { (Lower Back } \\
\text { Upper) }\end{array}$ & Decoupler/Row 2 & $\begin{array}{c}9.5264 \mathrm{e}-03 / \\
3.6243 \mathrm{e}-03\end{array}$ & $\begin{array}{c}\text { horizontal flow } \\
5 / 5\end{array}$ & 0.248 \\
\hline $\begin{array}{c}\text { Module } 14 \\
\text { (Lower Back } \\
\text { Lower) }\end{array}$ & Row 2 / Row 2 & $\begin{array}{c}3.6243 e-03 / \\
3.6243 e-03\end{array}$ & $\begin{array}{c}\text { horizontal flow } \\
5 / 5\end{array}$ & 0.248 \\
\hline $\begin{array}{c}\text { Module } 15 \\
\text { (Upper Front) }\end{array}$ & Row 2 / Row 2 & $\begin{array}{c}3.1132 \mathrm{e}-03 / \\
3.1132 \mathrm{e}-03\end{array}$ & $7 / 7$ & 0.521 \\
\hline $\begin{array}{c}\text { Module } 16 \\
\text { (Upper Lower) }\end{array}$ & Row 2 / Row 2 & $\begin{array}{c}3.1132 \mathrm{e}-03 / \\
3.1132 \mathrm{e}-03 \\
\end{array}$ & $7 / 7$ & 0.521 \\
\hline Total (bins) & & $\ldots$ & $141 / 169$ & \\
\hline
\end{tabular}

*unit - [m] 
TRAC BLANKET SYSTEM MODEL

Page:

Table 2.2-2 Typical area fractions for various types of blanket module bins.

\begin{tabular}{|c|c|c|c|c|}
\hline Row & Helium & Lead & Aluminum & H2O \\
\hline Row 1 & $10.7 \%$ & $42.7 \%$ & $22.4 \%$ & $24.2 \%$ \\
\hline Row 2 & $10.0 \%$ & $59.7 \%$ & $18.0 \%$ & $12.3 \%$ \\
\hline Row 3 & $9.5 \%$ & $65.0 \%$ & $18.0 \%$ & $7.5 \%$ \\
\hline
\end{tabular}

Table 2.2-3 Deposited powers, liquid flowrates and module coolant pipe sizes for 16 blanket modules.

\begin{tabular}{|c|c|c|c|}
\hline $\begin{array}{l}16 \text { Full Blanket } \\
\text { Modules } \\
\text { (Location) }\end{array}$ & $\begin{array}{l}\text { Power } \\
\text { downflow! } \\
\text { uptlow }\end{array}$ & $\begin{array}{l}\text { Liquid } \\
\text { Flowrate } \\
\text { (kg / sec) }\end{array}$ & $\begin{array}{l}\text { Module } \\
\text { Connection } \\
\text { Pipe Size (inch) }\end{array}$ \\
\hline $\begin{array}{c}\text { Module } 1 \\
\text { (Front } 1^{\text {st }} \text { Lateral) }\end{array}$ & $\begin{array}{l}3.869 \mathrm{MW} / \\
7.420 \mathrm{MW}\end{array}$ & 256 & 7.500 \\
\hline $\begin{array}{c}\text { Module } 2 \\
\text { (Front } 2^{\text {nd }} \text { Lateral) }\end{array}$ & $\begin{array}{c}0.765 \mathrm{MW} / \\
1.915 \mathrm{MW}\end{array}$ & 88 & 4.750 \\
\hline $\begin{array}{c}\text { Module } 3 \\
\text { (Front } 2^{\text {nd }} \text { Lateral) }\end{array}$ & $\begin{array}{l}0.765 \mathrm{MW} / \\
1.915 \mathrm{MW}\end{array}$ & 88 & 4.750 \\
\hline $\begin{array}{c}\text { Module } 4 \\
\text { (Back } 1^{\text {st }} \text { Lateral) }\end{array}$ & $\begin{array}{l}4.353 \mathrm{MW} / \\
8.348 \mathrm{MW}\end{array}$ & 288 & 8.000 \\
\hline $\begin{array}{c}\text { Module 5 } \\
\text { (Back 2 } 2^{\text {nd }} \text { Lateral) }\end{array}$ & $\begin{array}{l}0.765 \mathrm{MW} / \\
1.915 \mathrm{MW} \\
\end{array}$ & 88 & 4.750 \\
\hline $\begin{array}{c}\text { Module } 6 \\
\text { (Back } 2^{\text {nd }} \text { Lateral) }\end{array}$ & $\begin{array}{c}0.765 \mathrm{MW} / \\
1.915 \mathrm{MW}\end{array}$ & 88 & 4.750 \\
\hline $\begin{array}{c}\text { Module } 7 \\
\left(1^{\text {st }} \text { Downstream) }\right.\end{array}$ & $\begin{array}{l}0.744 \mathrm{MW} / \\
2.812 \mathrm{MW}\end{array}$ & 56 & 3.750 \\
\hline $\begin{array}{c}\text { Module } 8 \\
\left(2^{\text {nd }} \text { Downstream) }\right.\end{array}$ & $\begin{array}{l}3.924 \mathrm{MW} / \\
5.412 \mathrm{MW}\end{array}$ & 95 & 5.375 \\
\hline $\begin{array}{c}\text { Module } 9 \\
\left(3^{\text {rd }} \text { Downstream) }\right.\end{array}$ & $\begin{array}{l}1.355 \mathrm{MW} / \\
1.811 \mathrm{MW}\end{array}$ & 112 & 6.000 \\
\hline $\begin{array}{c}\text { Module } 10 \\
\text { (Blanket Upstream) }\end{array}$ & $\begin{array}{l}0.580 \mathrm{MW} / \\
1.240 \mathrm{MW}\end{array}$ & 154 & 6.000 \\
\hline $\begin{array}{c}\text { Module } 11 \\
\text { (Lower Front Upper) }\end{array}$ & $\begin{array}{l}0.225 \mathrm{MW} / \\
0.667 \mathrm{MW}\end{array}$ & 50 & 3.750 \\
\hline $\begin{array}{c}\text { Module } 12 \\
\text { (Lower Front Lower) }\end{array}$ & $\begin{array}{l}0.185 \mathrm{MW} / \\
0.050 \mathrm{MW}\end{array}$ & 40 & 3.500 \\
\hline $\begin{array}{c}\text { Module } 13 \\
\text { (Lower Back Upper) }\end{array}$ & $\begin{array}{l}0.268 \mathrm{MW} / \\
0.803 \mathrm{MW}\end{array}$ & 50 & 3.875 \\
\hline $\begin{array}{c}\text { Module } 14 \\
\text { (Lower Back Lower) }\end{array}$ & $\begin{array}{l}0.222 \mathrm{MW} / \\
0.060 \mathrm{MW}\end{array}$ & 40 & 3.625 \\
\hline $\begin{array}{l}\text { Module } 15 \\
\text { (Upper Front) }\end{array}$ & $\begin{array}{l}0.0 \mathrm{MW} / \\
0.706 \mathrm{MW}\end{array}$ & 38 & 2.750 \\
\hline $\begin{array}{c}\text { Module } 16 \\
\text { (Upper Back) }\end{array}$ & $\begin{array}{l}0.0 \mathrm{MW} / \\
0.706 \mathrm{MW}\end{array}$ & 38 & 2.750 \\
\hline Total Flowrate & $\begin{array}{l}18.785 \mathrm{MW} / \\
37.695 \mathrm{MW}\end{array}$ & 1569 & \\
\hline
\end{tabular}


TRAC BLANKET SYSTEM MODEL

Date:

$07 / 14 / 98$

Page:

Table 2.2-4 Decay power fractions of full power for lead cruciforms of various locations.

\begin{tabular}{|c|c|c|c|c|}
\hline Time & Lateral Row 1 & Lateral Row 2 & Lateral Row 3 & Downstream Row 1 \\
\hline 0 sec & $1.385 e-02$ & $2.079 \mathrm{e}-02$ & $2.801 \mathrm{e}-02$ & $9.736 \mathrm{e}-03$ \\
\hline $1 \mathrm{sec}$ & $1.209 \mathrm{e}-02$ & $1.747 \mathrm{e}-02$ & $2.370 \mathrm{e}-02$ & $8.718 \mathrm{e}-03$ \\
\hline $2 \mathrm{sec}$ & $1.142 \mathrm{e}-02$ & $1.614 \mathrm{e}-02$ & $2.197 \mathrm{e}-02$ & $8.335 \mathrm{e}-03$ \\
\hline $5 \mathrm{sec}$ & $1.086 \mathrm{e}-02$ & $1.511 \mathrm{e}-02$ & $2.061 \mathrm{e}-02$ & $8.000 \mathrm{e}-03$ \\
\hline $10 \mathrm{sec}$ & $1.059 \mathrm{e}-02$ & $1.477 \mathrm{e}-02$ & $2.014 \mathrm{e}-02$ & $7.836 \mathrm{e}-03$ \\
\hline $20 \mathrm{sec}$ & $1.024 \mathrm{e}-02$ & $1.432 \mathrm{e}-02$ & $1.947 \mathrm{e}-02$ & $7.620 \mathrm{e}-03$ \\
\hline $1 \mathrm{~min}$. & $9.237 \mathrm{e}-03$ & $1.293 \mathrm{e}-02$ & $1.730 \mathrm{e}-02$ & $7.035 \mathrm{e}-03$ \\
\hline $2 \mathrm{~min}$. & $8.125 \mathrm{e}-03$ & $1.135 \mathrm{e}-02$ & $1.482 \mathrm{e}-02$ & $6.399 \mathrm{e}-03$ \\
\hline $5 \mathrm{~min}$. & $6.215 \mathrm{e}-03$ & $8.651 \mathrm{e}-03$ & $1.059 \mathrm{e}-02$ & $5.280 \mathrm{e}-03$ \\
\hline $10 \mathrm{~min}$. & $5.058 \mathrm{e}-03$ & $7.042 \mathrm{e}-03$ & $8.183 \mathrm{e}-03$ & $4.520 \mathrm{e}-03$ \\
\hline $20 \mathrm{~min}$. & $4.454 \mathrm{e}-03$ & $6.255 \mathrm{e}-03$ & $7.174 \mathrm{e}-03$ & $3.998 \mathrm{e}-03$ \\
\hline $1 \mathrm{hr}$. & $3.849 \mathrm{e}-03$ & $5.490 \mathrm{e}-03$ & $6.370 \mathrm{e}-03$ & $3.346 \mathrm{e}-03$ \\
\hline $2 \mathrm{hr}$. & $3.457 \mathrm{e}-03$ & $4.950 \mathrm{e}-03$ & $5.783 \mathrm{e}-03$ & $2.938 \mathrm{e}-03$ \\
\hline 5 hr. & $2.831 \mathrm{e}-03$ & $4.062 \mathrm{e}-03$ & $4.784 \mathrm{e}-03$ & $2.363 \mathrm{e}-03$ \\
\hline $10 \mathrm{hr}$. & $2.267 \mathrm{e}-03$ & $3.271 \mathrm{e}-03$ & $3.872 \mathrm{e}-03$ & $1.898 \mathrm{e}-03$ \\
\hline 1 day & $1.472 \mathrm{e}-03$ & $2.169 \mathrm{e}-03$ & $2.573 \mathrm{e}-03$ & $1.284 \mathrm{e}-03$ \\
\hline 2 days & $9.137 \mathrm{e}-04$ & $1.377 \mathrm{e}-03$ & $1.618 \mathrm{e}-03$ & $8.591 \mathrm{e}-04$ \\
\hline 5 days & $4.449 \mathrm{e}-04$ & $6.317 \mathrm{e}-04$ & $6.990 \mathrm{e}-04$ & $4.776 \mathrm{e}-04$ \\
\hline 10 days & $2.677 \mathrm{e}-04$ & $3.457 \mathrm{e}-04$ & $3.595 \mathrm{e}-04$ & $3.075 \mathrm{e}-04$ \\
\hline 20 days & $1.664 \mathrm{e}-04$ & $2.046 \mathrm{e}-04$ & $2.088 \mathrm{e}-04$ & $1.963 \mathrm{e}-04$ \\
\hline 30 days & $1.239 \mathrm{e}-04$ & $1.488 \mathrm{e}-04$ & $1.516 \mathrm{e}-04$ & $1.475 \mathrm{e}-04$ \\
\hline 60 days & $7.690 \mathrm{e}-04$ & $8.841 \mathrm{e}-04$ & $9.016 \mathrm{e}-05$ & $9.167 \mathrm{e}-05$ \\
\hline
\end{tabular}


TRAC BLANKET SYSTEM MODEL

Page:

Table 2.4-1 6 lumped blanket module system model used for the present PSAR analysis

\begin{tabular}{|c|c|c|c|}
\hline $\begin{array}{l}6 \text { Lumped } \\
\text { Modules }\end{array}$ & $\begin{array}{l}\text { Prototypic } 16 \text { Fuil Blanket } \\
\text { Modules }\end{array}$ & $\begin{array}{l}\text { Thermal Deposited Power } \\
\text { Downilow / Uptlow / Total } \\
\text { Power }\end{array}$ & $\begin{array}{l}\text { Plpe } \\
\text { Size: } \\
\text { (inch) }\end{array}$ \\
\hline Module 1 & $\begin{array}{l}\text { Front } 1^{\text {st }} \text { Lateral Dec. / Row } 1 \\
\text { Module } \\
\text { Back } 1^{\text {st }} \text { Lateral Dec. / Row } 1 \\
\text { Module }\end{array}$ & $\begin{array}{c}8.222 \mathrm{MW} / 15.768 \mathrm{MW} / \\
23.990 \mathrm{MW}\end{array}$ & 7.500 \\
\hline Module 2 & $\begin{array}{l}\text { Front } 2^{\text {nd }} \text { Lateral Row } 2 / \text { Row } 3 \\
\text { Modules } \\
\text { Back } 2^{\text {nd }} \text { Lateral Row } 2 / \text { Row } 3 \\
\text { Modules }\end{array}$ & $\begin{array}{c}3.060 \mathrm{MW} / 7.660 \mathrm{MW} / 10.720 \\
\mathrm{MW}\end{array}$ & 4.750 \\
\hline Module 3 & $\begin{array}{l}1^{\text {st }} \text { Downstream Dec. / Row } 1 \\
\text { Module }\end{array}$ & $\begin{array}{c}0.744 \mathrm{MW} / 2.812 \mathrm{MW} / 3.556 \\
\mathrm{MW}\end{array}$ & 3.750 \\
\hline Module 4 & $\begin{array}{l}2^{\text {nd }} \text { Downstream Row } 1 \text { / Row } 2 \\
\text { Module }\end{array}$ & $\begin{array}{c}3.924 \mathrm{MW} / 5.412 \mathrm{MW} / 9.336 \\
\mathrm{MW}\end{array}$ & 5.375 \\
\hline Module 5 & $\begin{array}{c}3^{\text {rd }} \text { Downstream Row } 2 / \text { Row } 2 \\
\text { Module }\end{array}$ & $\begin{array}{c}1.355 \mathrm{MW} / 1.811 \mathrm{MW} / 3.167 \\
\mathrm{MW}\end{array}$ & 6.000 \\
\hline Module 6 & $\begin{array}{c}\text { Low Power Modules } \\
\text { Blanket Upstream Dec. / Row } 2 \\
\text { Module } \\
\text { Lower Front Dec. / Row } 2 \text { Module } \\
\text { Lower Front Row } 2 \text { / Row } 2 \text { Module } \\
\text { Lower Back Dec. / Row } 2 \text { Module } \\
\text { Lower Back Row } 2 \text { / Row } 2 \text { Module } \\
\text { Upper Front Row } 2 \text { / Row } 2 \text { Module } \\
\text { Upper Back Row } 2 \text { / Row } 2 \text { Module }\end{array}$ & $\begin{array}{c}\text { (Horizontal Flow) } \\
\mathrm{omW} / 5.712 \mathrm{MW} / 5.712 \mathrm{MW}\end{array}$ & 3.875 \\
\hline $\begin{array}{l}\text { Total } \\
\text { Deposited } \\
\text { Power }\end{array}$ & & $\begin{array}{c}17.305 \mathrm{MW} / 39.175 \mathrm{MW} / \\
56.480 \mathrm{MW}\end{array}$ & \\
\hline
\end{tabular}


TRAC BLANKET SYSTEM MODEL

Table 2.4-2 Blanket System Component Descriptions in TRAC Model.

\begin{tabular}{|c|c|c|c|c|}
\hline System & Component Type & Comp & $\begin{array}{l}\text { No of } \\
\text { Cells }\end{array}$ & Descriptions \\
\hline \multirow[t]{46}{*}{$\mathrm{HR}$} & Fixed Header (FH) & 380 & 1 & coolant Supply FH \\
\hline & & 340 & 1 & coolant Return FH \\
\hline & Pressurizer (Pzr) & 760 & 1 & Pzr surge line 1 connected to Supply FH 380 \\
\hline & & 761 & 2 & Pzr surge line 2 \\
\hline & & 762 & 1 & Pzr surge line 3 \\
\hline & & 763 & 1 & Pzr surge line 4 \\
\hline & & 764 & 1 & Pzr surge line 5 \\
\hline & & 765 & 13 & Pzr surge line 6 \\
\hline & & 766 & 9 & primary Pzr \\
\hline & Hot Leg Loop & 20 & 1 & pipe connected to Return FH340 \\
\hline & & 21 & 1 & plenum for potential break loc. \\
\hline & & 22 & 7 & pipe connection to external loop \\
\hline & & 23 & 1 & pipe connect. for potential break \\
\hline & & 24 & 13 & connection pipe \\
\hline & & 25 & 1 & connection pipe \\
\hline & & 26 & 2 & pipe connected to two pumps \\
\hline & & 27 & 1 & plenum for two pump connection \\
\hline & & 28 & 2 & pump\#1 suction pipe \\
\hline & & 29 & 7 & pump\#2 suction pipe \\
\hline & & 30 & 2 & pump located at cell face 2 \\
\hline & & 31 & 2 & pump located at cell face 2 \\
\hline & & 32 & 3 & check valve located at pump\#1 discharge \\
\hline & & 33 & 3 & check valve located at pump\#2 discharge \\
\hline & & 34 & 1 & pump outlet plenum \\
\hline & & 36 & 1 & connect. pipe between pump and pipe \\
\hline & & 37 & 1 & HX connect. pipe for potential break \\
\hline & & 38 & 1 & pipe connect. to two HX's inlet plenum \\
\hline & & 40 & 1 & plenum \\
\hline & $H X$ & 48 & 3 & HX \#1 inlet pipe \\
\hline & . & 50 & 4 & $H X \# 11^{\text {st }}$ pass \\
\hline & & 52 & 3 & HX \#1 middle header \\
\hline & & 54 & 4 & $\mathrm{HX} \# 12^{\text {nd }}$ pass \\
\hline & & 49 & 3 & $H X \# 2$ inlet pipe \\
\hline & & 51 & 4 & 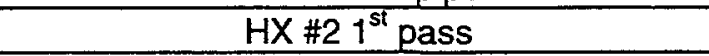 \\
\hline & & 53 & 3 & $\mathrm{HX} \# 2$ middle header \\
\hline & & 55 & 4 & $\mathrm{HX} \# 22^{\text {nd }}$ pass \\
\hline & & 710 & 1 & HX \#1 secondary side fill \\
\hline & & 711 & 4 & $\mathrm{HX} \# 12^{\text {nd }}$ pass secondary side \\
\hline & & 712 & 3 & HX \#1 middle header secondary side \\
\hline & & 713 & 4 & $\mathrm{HX} \# 11^{\text {st }}$ pass secondary side \\
\hline & & 714 & 1 & $\mathrm{HX} \# 1$ secondary side break $\mathrm{BC}$ \\
\hline & & 730 & 1 & $\mathrm{HX} \# 2$ secondary side fill $\mathrm{BC}$ \\
\hline & & 731 & 4 & $\mathrm{HX} \# 22^{\text {nd }}$ pass secondary side \\
\hline & & 732 & 3 & $\mathrm{HX} \# 2$ middle header secondary side \\
\hline & & 733 & 4 & $H X \# 21^{\text {st }}$ pass secondary side \\
\hline & & 734 & 1 & $\mathrm{HX} \# 2$ secondary side break BC \\
\hline
\end{tabular}


TRAC BLANKET SYSTEM MODEL

Page:

Table 2.4-2 Blanket System Component Descriptions in TRAC Model (continued).

\begin{tabular}{|c|c|c|c|c|}
\hline System & Component Type & Comp & $\begin{array}{l}\text { No } \\
\text { of } \\
\text { Cells }\end{array}$ & Descriptions \\
\hline \multirow[t]{12}{*}{$\mathrm{HR}$} & Cold Leg Loop & 56 & 3 & $\mathrm{HX} \# 1$ outlet pipe \\
\hline & & 57 & 6 & $\mathrm{HX} \# 2$ outlet pipe \\
\hline & & 60 & 3 & $\mathrm{HX}$ outlet plenum merged after two $\mathrm{HX}$ 's \\
\hline & & 62 & 1 & cold leg pipe \\
\hline & & 63 & 1 & cold leg pipe \\
\hline & & 64 & 13 & cold leg pipe located outside cavity wall \\
\hline & & 65 & 1 & pipe for cold leg pipe break \\
\hline & & 66 & 1 & horizontal cold leg pipe penetration \\
\hline & & 67 & 1 & plenum for internal break on HR loop \\
\hline & & 854 & 2 & HR isolation valve for internal break \\
\hline & & 69 & 1 & plenum for internal LOCA simulation \\
\hline & & 68 & 5 & pipe connect. to FH 340 inside cavity \\
\hline \multirow[t]{9}{*}{$\begin{array}{l}\text { Cavity } \\
\text { Vessel } \\
\end{array}$} & Cold Leg Loop & 850 & 2 & valve located bet. cavity vessel and $\mathrm{HR}$ \\
\hline & & 852 & 2 & valve located bet. cavity vessel and $H R$ \\
\hline & & & 1 & plenum for cavity vessel connection \\
\hline & & 828 & 3 & cavity vent valve \\
\hline & & 802 & 1 & break component for cavity vent pressure BC \\
\hline & & 823 & 11 & pipe for cavity lower section simulation \\
\hline & & 824 & 1 & plenum for cavity connection \\
\hline & & 840 & 2 & valve to connect cavity line to Module 1 \\
\hline & & 825 & 4 & pipe for cavity middle section simulation \\
\hline \multirow[t]{8}{*}{$\begin{array}{l}\text { Cavity } \\
\text { Pool }\end{array}$} & Cavity Flood Line & 820 & 13 & $\begin{array}{l}\text { pipe for cavity pool connection to cavity } \\
\text { vessel }\end{array}$ \\
\hline & & 821 & 2 & cavity flood line valve \\
\hline & & 822 & 1 & flood line pipe inside cavity vessel \\
\hline & Cavity Flood Pool & 801 & 1 & break component for cavity pool BC \\
\hline & & 810 & 10 & pipe for top cavity pool section \\
\hline & & 811 & 1 & plenum for middle cavity pool section \\
\hline & & 812 & 7 & pipe for lower cavity pool section \\
\hline & & 813 & 1 & plenum for cavity pool bottom \\
\hline \multirow[t]{14}{*}{$\mathrm{RHR}$} & RHR Loop & 621 & 1 & pipe located to return FH \\
\hline & & 623 & 10 & pipe located inside the cavity vessel \\
\hline & & 624 & 1 & pipe located outside the cavity vessel \\
\hline & & 625 & 18 & pipe bet. RHR pump and pipe comp. \#624 \\
\hline & & 630 & 2 & RHR pump located at face 2 \\
\hline & & 640 & 3 & check valve located at pump discharge \\
\hline & & 652 & 4 & HX tubes \\
\hline & & 660 & 16 & pipe at the cold leg side \\
\hline & & 661 & 1 & pipe located before cavity vessel \\
\hline & & 662 & 8 & cold leg pipe inside cavity vessel \\
\hline & & 663 & 1 & cold leg pipe connected to supply FH \\
\hline & & 672 & 1 & fill for $\mathrm{HX}$ secondary side $\mathrm{BC}$ \\
\hline & & 671 & 4 & HX secondary shell side \\
\hline & & 673 & 1 & break comp. for $\mathrm{HX}$ secondary side $\mathrm{BC}$ \\
\hline
\end{tabular}


TRAC BLANKET SYSTEM MODEL

Table 2.4-2 Blanket System Component Descriptions in TRAC Model (continued).

\begin{tabular}{|c|c|c|c|c|}
\hline System & Component Type & Comp & $\begin{array}{l}\text { No } \\
\text { of } \\
\text { Cells }\end{array}$ & Descriptions \\
\hline \multirow[t]{36}{*}{ Module } & Module 1 Flow & 454 & 7 & pipe connected to supply FH \\
\hline & & 80 & 1 & $\begin{array}{l}\text { plenum for potential internal break simulation } \\
\text { at Module } 1\end{array}$ \\
\hline & & 375 & 5 & $\begin{array}{c}\text { pipe connection bet. Suuply FH and Module } 1 \\
\text { upper plenum }\end{array}$ \\
\hline & & 370 & 1 & upper plenum for Module 1 downcomer \\
\hline & & 360 & 5 & Module 1 downflow region \\
\hline & & 350 & 1 & $\begin{array}{c}\text { middle plenum bet. Module } 1 \text { downflow and } \\
\text { upflow regions }\end{array}$ \\
\hline & & 300 & 5 & Module 1 upflow region \\
\hline & & 330 & 1 & upper plenum for module 1 upflow region \\
\hline & & 335 & 5 & connection pipe after Module 1 upper plenum \\
\hline & & 429 & 4 & pipe connected to return $\mathrm{FH}$ \\
\hline & Module 2 Flow & 173 & 7 & pipe coonected to supply $\mathrm{FH}$ \\
\hline & & 81 & 1 & $\begin{array}{l}\text { plenum for potential internal break simulation } \\
\text { at Module } 2\end{array}$ \\
\hline & & 82 & 3 & pipe connection \\
\hline & & 172 & 1 & upper plenum for Module 2 downcomer \\
\hline & & 158 & 6 & Module 2 downflow region \\
\hline & & 147 & 1 & $\begin{array}{c}\text { middle plenum bet. Module } 2 \text { downflow and } \\
\text { upflow regions }\end{array}$ \\
\hline & & 102 & 6 & Module 2 upflow region \\
\hline & & 133 & 1 & upper plenum for module 2 upflow region \\
\hline & & 136 & 7 & pipe connected to return FH \\
\hline & Module 3 Flow & 415 & 7 & pipe connected to supply FH \\
\hline & & 85 & 1 & $\begin{array}{l}\text { plenum for potential internal break simulation } \\
\text { at Module } 3\end{array}$ \\
\hline & & 86 & 3 & pipe connection \\
\hline & & 479 & 1 & upper plenum for Module 3 downcomer \\
\hline & & 478 & 5 & Module 3 downflow region \\
\hline & & 418 & 1 & $\begin{array}{c}\text { middle plenum bet. Module } 3 \text { downflow and } \\
\text { upflow regions }\end{array}$ \\
\hline & & 409 & 5 & Module 3 upflow region \\
\hline & & 423 & 1 & upper plenum for module 3 upflow region \\
\hline & & 417 & 7 & pipe connected to return $\mathrm{FH}$ \\
\hline & Module 4 Flow & 485. & 7 & pipe connected to supply FH \\
\hline & & 87 & 1 & $\begin{array}{l}\text { plenum for potential internal break simulation } \\
\text { at Module } 4\end{array}$ \\
\hline & & 88 & 3 & pipe connection \\
\hline & & 489 & 1 & upper plenum for Module 4 downcomer \\
\hline & & 480 & 6 & Module 4 downflow region \\
\hline & & 419 & 1 & $\begin{array}{c}\text { middle plenum bet. Module } 4 \text { downflow and } \\
\text { upflow regions }\end{array}$ \\
\hline & & 412 & 6 & Module 4 upflow region \\
\hline & & 483 & 1 & r plenum for module 4 upflow region \\
\hline
\end{tabular}


TRAC BLANKET SYSTEM MODEL

Page:

Table 2.4-2 Blanket System Component Descriptions in TRAC Model (continued).

\begin{tabular}{|c|c|c|c|c|}
\hline System & Component Type & Comp & $\begin{array}{l}\text { No } \\
\text { of } \\
\text { Cells }\end{array}$ & Descriptions \\
\hline & & 484 & 7 & pipe connected to return $\mathrm{FH}$ \\
\hline \multirow[t]{21}{*}{ Module } & Module 5 Flow & $51: 3$ & 7 & pipe connected to supply FH \\
\hline & & 89 & 1 & $\begin{array}{c}\text { plenum for potential internal break simulation } \\
\text { at Module } 5\end{array}$ \\
\hline & & 90 & 3 & pipe connection \\
\hline & & 510 & 1 & upper plenum for Module 5 downcomer \\
\hline & & 507 & 6 & Module 5 downflow region \\
\hline & & 503 & 1 & $\begin{array}{l}\text { middle plenum bet. Module } 4 \text { downflow and } \\
\text { upflow regions }\end{array}$ \\
\hline & & 500 & 6 & Module 5 upflow region \\
\hline & & 508 & 1 & upper plenum for Module 5 upflow region \\
\hline & & 511 & 7 & pipe connected to return FH \\
\hline & Module 6 Flow & 541 & 7 & pipe connected to supply FH \\
\hline & & 83 & 1 & $\begin{array}{c}\text { plenum for potential internal break simulation } \\
\text { at Module } 6\end{array}$ \\
\hline & & 84 & 1 & pipe connection \\
\hline & & 538 & 1 & upper plenum for Module 6 decoupler \\
\hline & & 535 & 5 & Module 6 downcomer region \\
\hline & & 531 & 1 & $\begin{array}{l}\text { middle plenum bet. Module } 6 \text { decoupler and } \\
\text { main heated regions }\end{array}$ \\
\hline & & 528 & 5 & Module 6 main heated region \\
\hline & & 536 & 1 & $\begin{array}{c}\text { upper plenum for module } 6 \text { main heated } \\
\text { region }\end{array}$ \\
\hline & & 539 & 12 & pipe connected to return $\mathrm{FH}$ \\
\hline & $\begin{array}{l}\text { Module } 1 \text { Heater } \\
\text { Structure }\end{array}$ & 901 & 5 & Al tube structure in Row 1 \\
\hline & & 951 & 5 & Lead zone with Al cladding in Row 1 \\
\hline & & 984 & 5 & Al tube structure in decoupler \\
\hline \multirow[t]{13}{*}{ - } & $\begin{array}{l}\text { Module } 2 \text { Heater } \\
\text { Structure }\end{array}$ & 905 & 6 & Al tube structure in Row 2 \\
\hline & & 955 & 6 & Lead zone with Al cladding in Row 2 \\
\hline & & 916 & 6 & Al tube structure in Row 3 \\
\hline & & 966 & 6 & Lead zone with Al cladding in Row 3 \\
\hline & $\begin{array}{l}\text { Module } 3 \text { Heater } \\
\text { Structure }\end{array}$ & 911 & 5 & Al tube structure in Row 1 \\
\hline & & 961 & 5 & Lead zone with Al cladding in Row 1 \\
\hline & & 988 & 5 & Al tube structure in decoupler \\
\hline & $\begin{array}{l}\text { Module } 4 \text { Heater } \\
\text { Structure }\end{array}$ & 912 & 6 & Al tube structure in Row 1 \\
\hline & & 962 & 6 & Lead zone with Al cladding in Row 1 \\
\hline & & 931 & 6 & Al tube structure in Row 2 \\
\hline & & 978 & 6 & Lead zone with Al cladding in Row 2 \\
\hline & $\begin{array}{l}\text { Module } 5 \text { Heater } \\
\text { Structure }\end{array}$ & 913 & 6 & Al tube structure in Row 2 \\
\hline & & 963 & 6 & Lead zone with Al cladding in Row 2 \\
\hline
\end{tabular}


TRAC BLANKET SYSTEM MODEL

Page:

Table 2.4-2 Blanket System Component Descriptions in TRAC Model (continued).

\begin{tabular}{|c|c|c|c|c|}
\hline System & Component Type & Comp & $\begin{array}{c}\text { No } \\
\text { of } \\
\text { Cells }\end{array}$ & Descriptions \\
\hline & & 932 & 6 & Al tube structure in Row 2 \\
\hline & & 979 & 6 & Lead zone with Al cladding in Row 2 \\
\hline & $\begin{array}{c}\text { Module 6 Heater } \\
\text { Structure }\end{array}$ & 915 & 5 & Al tube structure in Row 2 \\
\hline & & 965 & 5 & Lead zone with Al cladding in Row 2 \\
\hline
\end{tabular}

Table 2.4-3 Steady State Conditions.

\begin{tabular}{|c|c|c|}
\hline & Parameter & $\begin{array}{c}\text { Calculated } \\
\text { Values }\end{array}$ \\
\hline Total power deposited in blanket modules & $\mathrm{MW}$ & 56.5 \\
\hline Total flow rate & $\mathrm{kg} / \mathrm{sec}$ & 1569 \\
& $\mathrm{gpm}$ & 25252 \\
\hline Pressure in cold-leg fixed header & Mpa & 0.7325 \\
& psia & 106.24 \\
\hline Pressure in hot-leg fixed header & Mpa & 0.4563 \\
& psia & 66.180 \\
\hline Pressurizer (cell \#1) pressure & Mpa & 0.7311 \\
& psia & 106.03 \\
\hline Pump \#1 suction pressure & Mpa & 0.2751 \\
& psia & 39.90 \\
\hline Pump \#1 discharge pressure & Mpa & 1.0356 \\
& psia & 150.20 \\
\hline Pump \#2 suction pressure & Mpa & 0.2958 \\
& psia & 42.91 \\
\hline Pump \#2 discharge pressure & Mpa & 1.0409 \\
& psia & 150.97 \\
\hline Temperature in cold-leg fixed header & $\mathrm{C}$ & 49.43 \\
& $\mathrm{~F}$ & 121.0 \\
\hline Temperature in hot-leg fixed header & $\mathrm{C}$ & 58.03 \\
& $\mathrm{~F}$ & 136.5 \\
\hline Max. fluid temperature of the hottest & $\mathrm{C}$ & 71.95 \\
module & $\mathrm{F}$ & 161.5 \\
\hline
\end{tabular}


Table 2.4-4 6 Lumped Blanket Modules from 16 Full Blanket Module System.

\begin{tabular}{|c|c|c|c|c|c|}
\hline \multirow{2}{*}{$\begin{array}{l}6 \text { Modules } \\
\end{array}$} & \multirow[b]{2}{*}{16 ruI Blanket } & \multicolumn{2}{|c|}{ Mass Flow Rate } & \multicolumn{2}{|c|}{$(\Delta T)_{100}$} \\
\hline & & $\begin{array}{l}\text { (kg) } \\
(\mathrm{kgls})\end{array}$ & $\begin{array}{l}\text { Model } \\
\text { (kglsec) }\end{array}$ & $\begin{array}{c}(\mathrm{CD} \\
(\mathrm{Cl})\end{array}$ & $\begin{array}{c}\text { Model } \\
(\mathrm{c})\end{array}$ \\
\hline Module 1 & $\begin{array}{c}2 \text { First-Row Lateral } \\
\text { Modules }\end{array}$ & 544 & 544 & 10.5 & 10.4 \\
\hline Module 2 & $\begin{array}{c}4 \text { Second-Row Lateral } \\
\text { Modules }\end{array}$ & 352 & 344 & 7.27 & 7.48 \\
\hline Module 3 & $\begin{array}{c}\text { First Downstream } \\
\text { Module (Dec. / Row 1) }\end{array}$ & 56 & 55 & 15.2 & 15.7 \\
\hline Module 4 & $\begin{array}{c}\text { Second Downstream } \\
\text { Module (Row } 1 / \text { Row } 2 \text { ) }\end{array}$ & 95 & 99 & 23.4 & 22.6 \\
\hline Module 5 & $\begin{array}{c}\text { Third Downstream } \\
\text { Module (Row 2/ Row 2) }\end{array}$ & 112 & 117 & 6.75 & 6.51 \\
\hline Module 6 & $\begin{array}{l}\text { Upstream Module } \\
4 \text { Bottom Modules } \\
2 \text { Top Modules }\end{array}$ & 410 & 410 & 3.32 & 3.34 \\
\hline $\begin{array}{l}\text { Overall } \\
\text { System }\end{array}$ & & 1569 & 1569 & 8.60 & 8.59 \\
\hline
\end{tabular}

$(\Delta P)_{\text {Fixed Header }}=40.15$ psid for 6 blanket module system model 


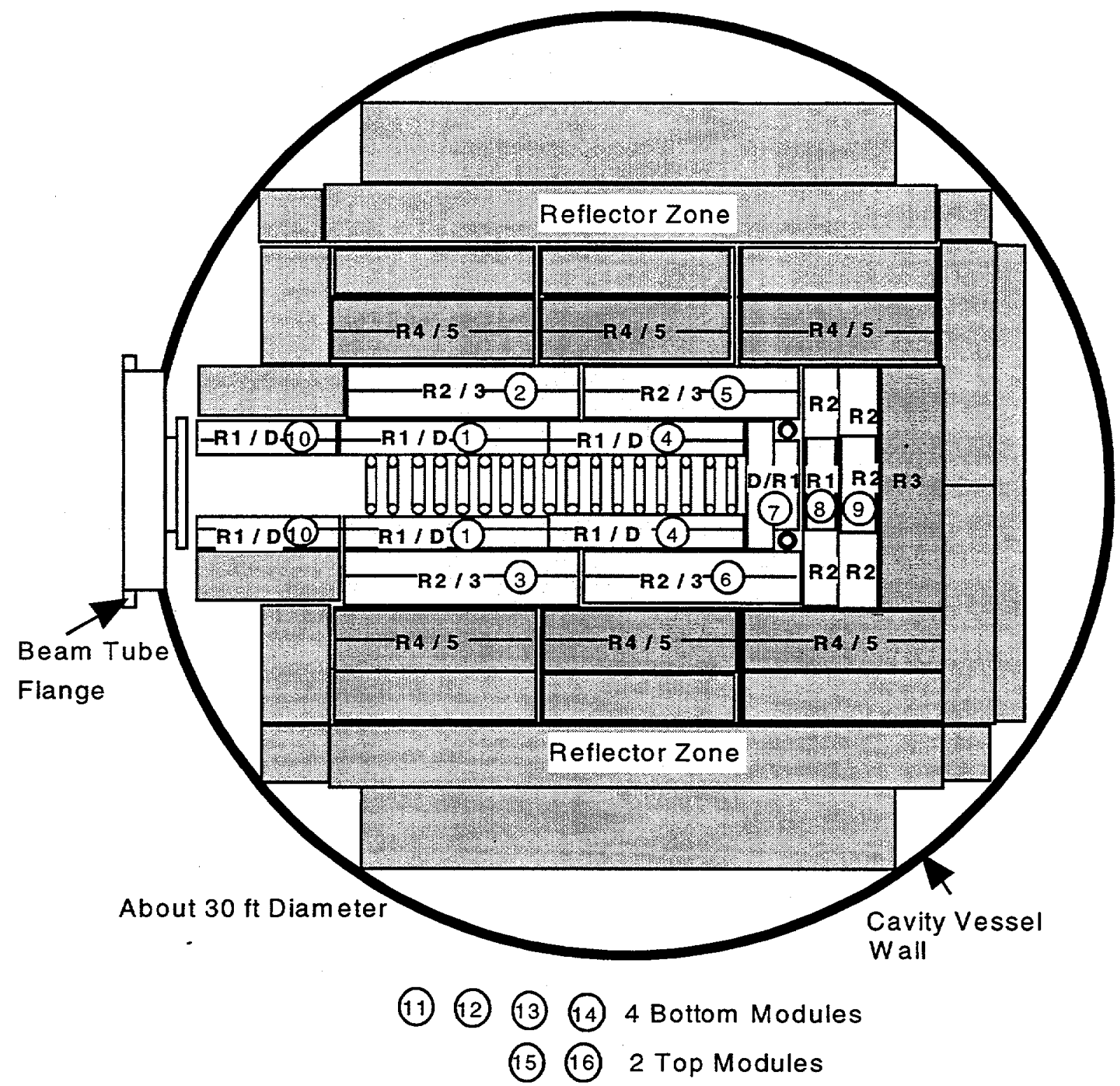

Figure 2.2-1 Top cross-sectional facemap of 16 blanket system modules. 


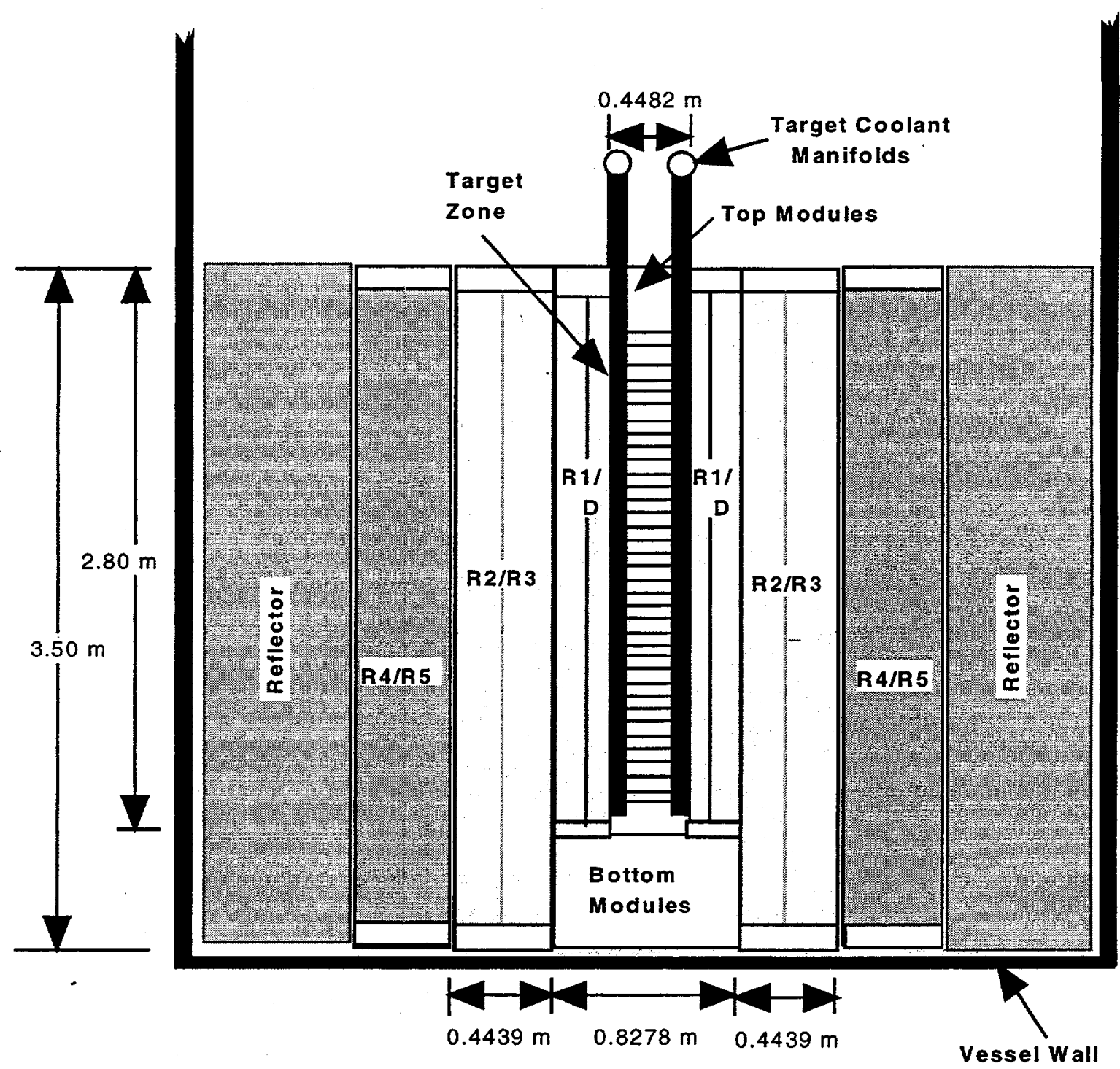

Figure 2.2-2 Front cross sectional view of the blanket system modules. 


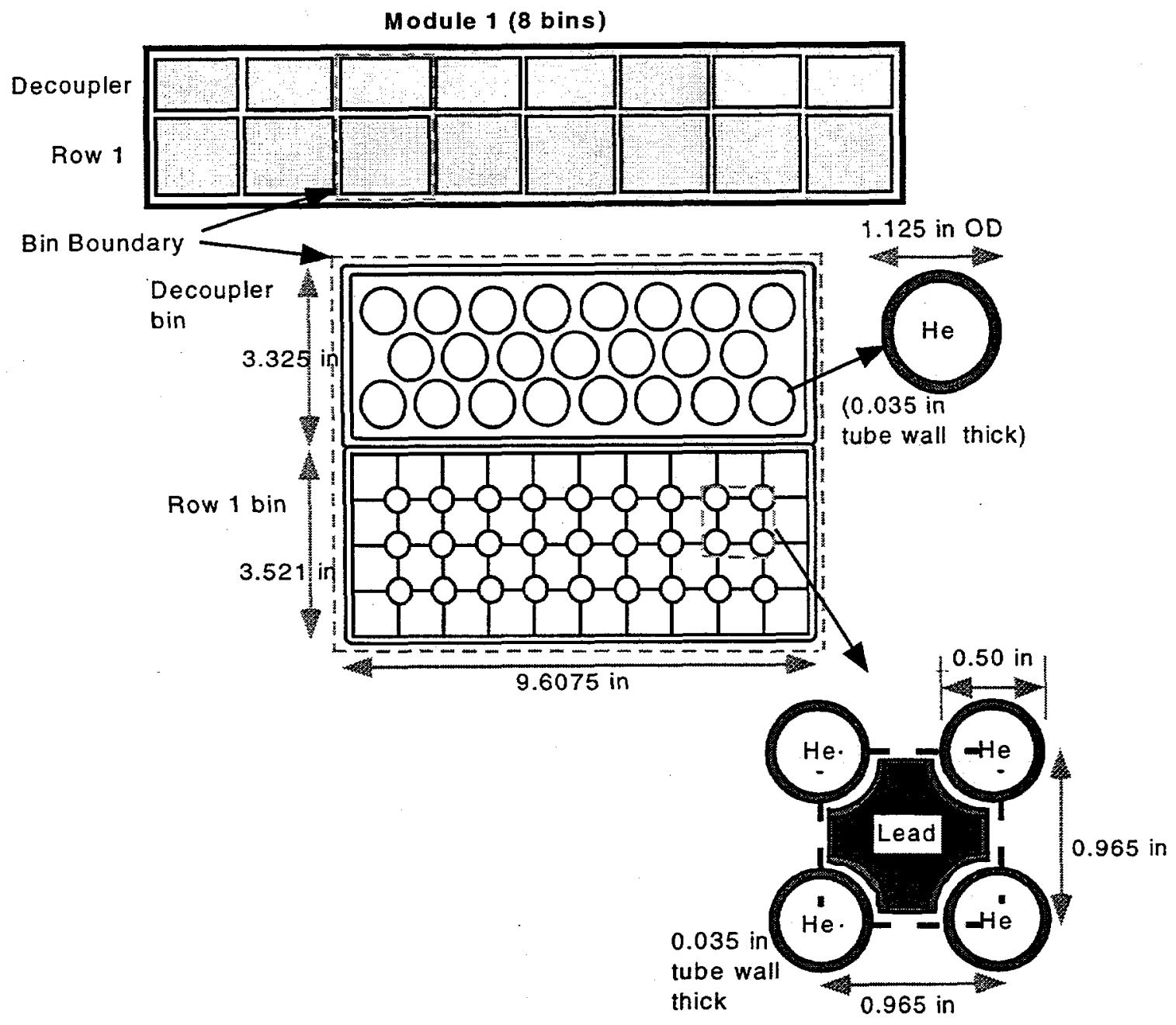

Figure 2.2-3 Decoupler and Row 1 cruciform bin geometries. 


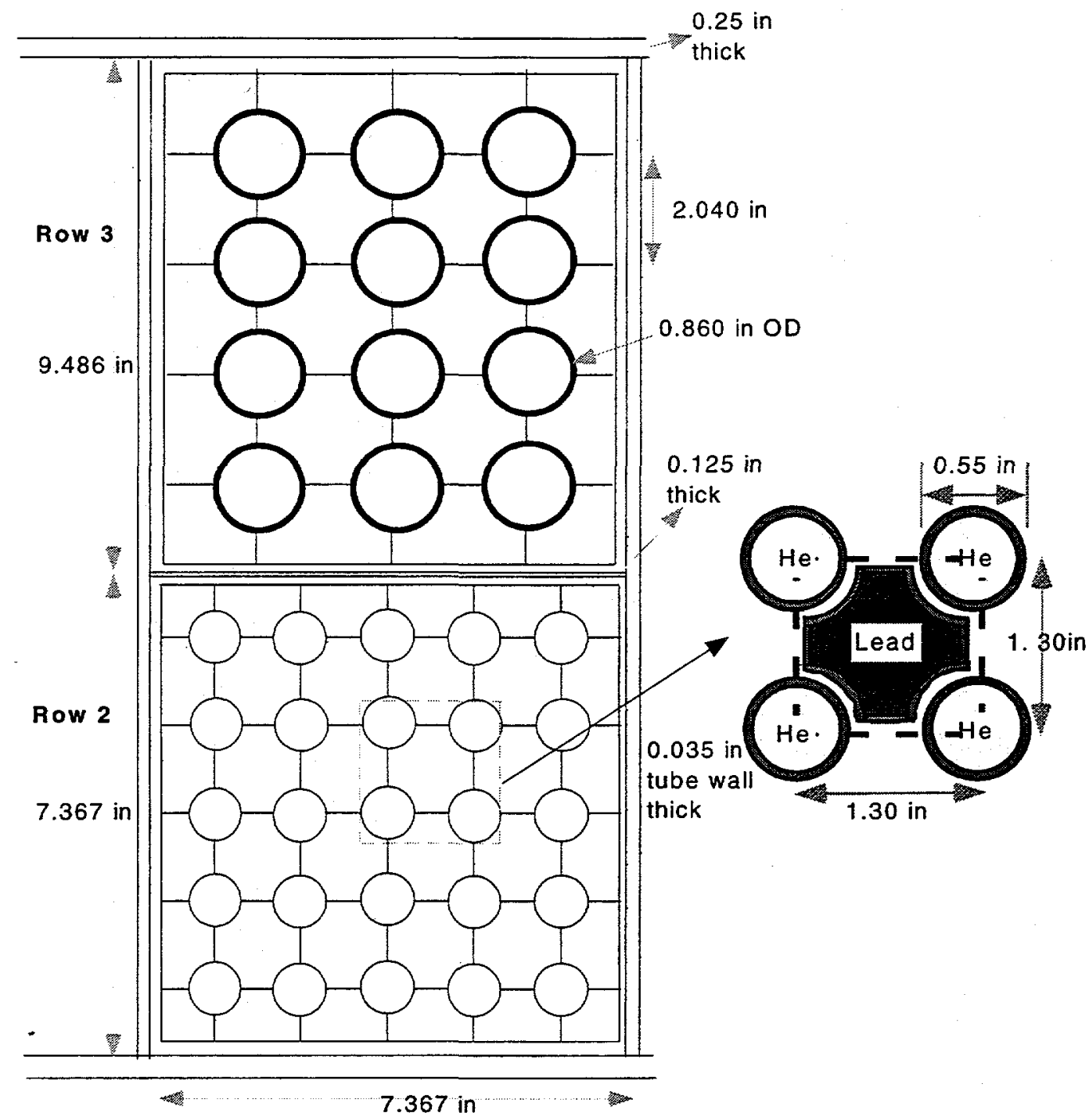

Figure 2.2-4 Row 2 and Row 3 cruciform bin geometries. 


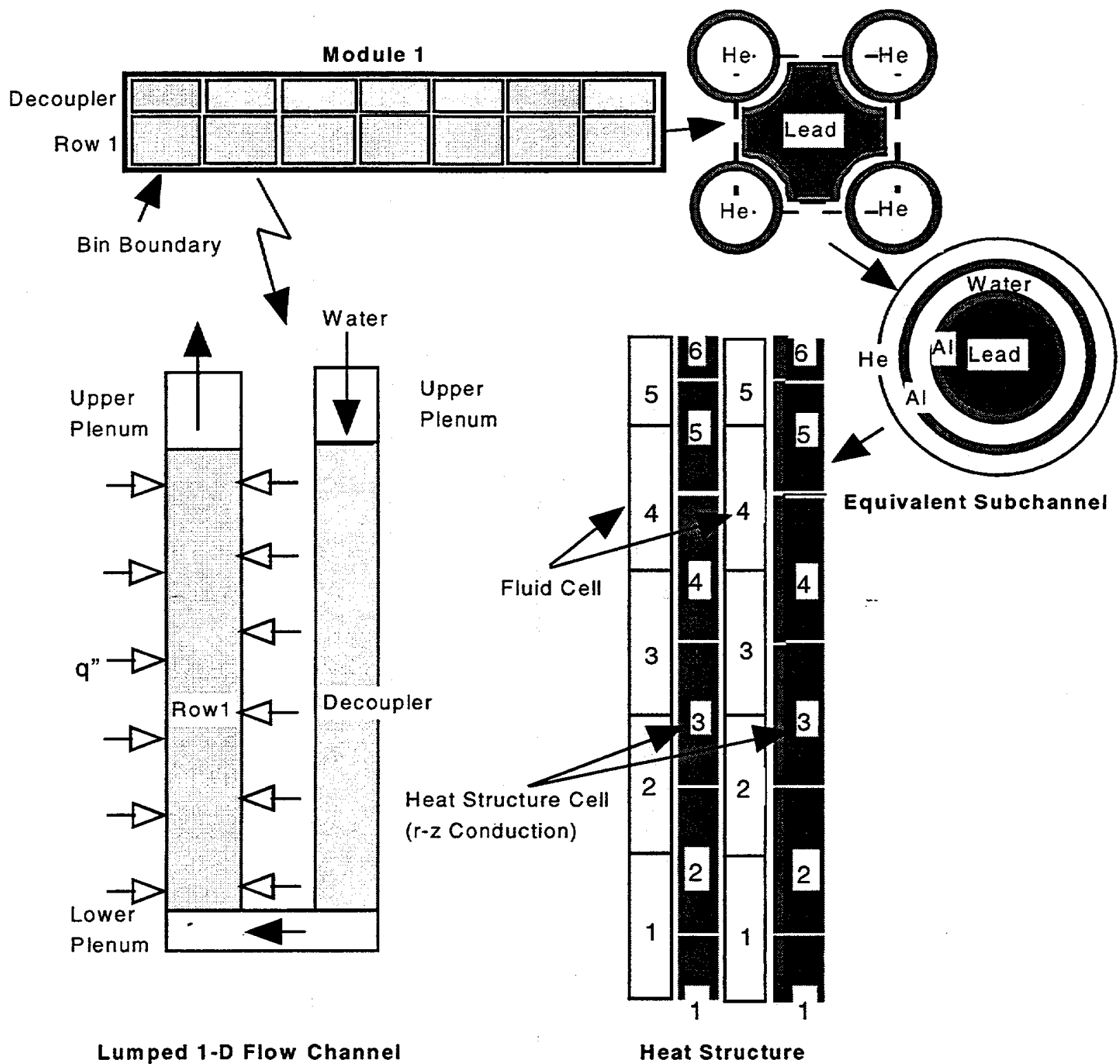

Figure 2.3-1 Lumping strategy for 1-D TRAC blanket system model. 


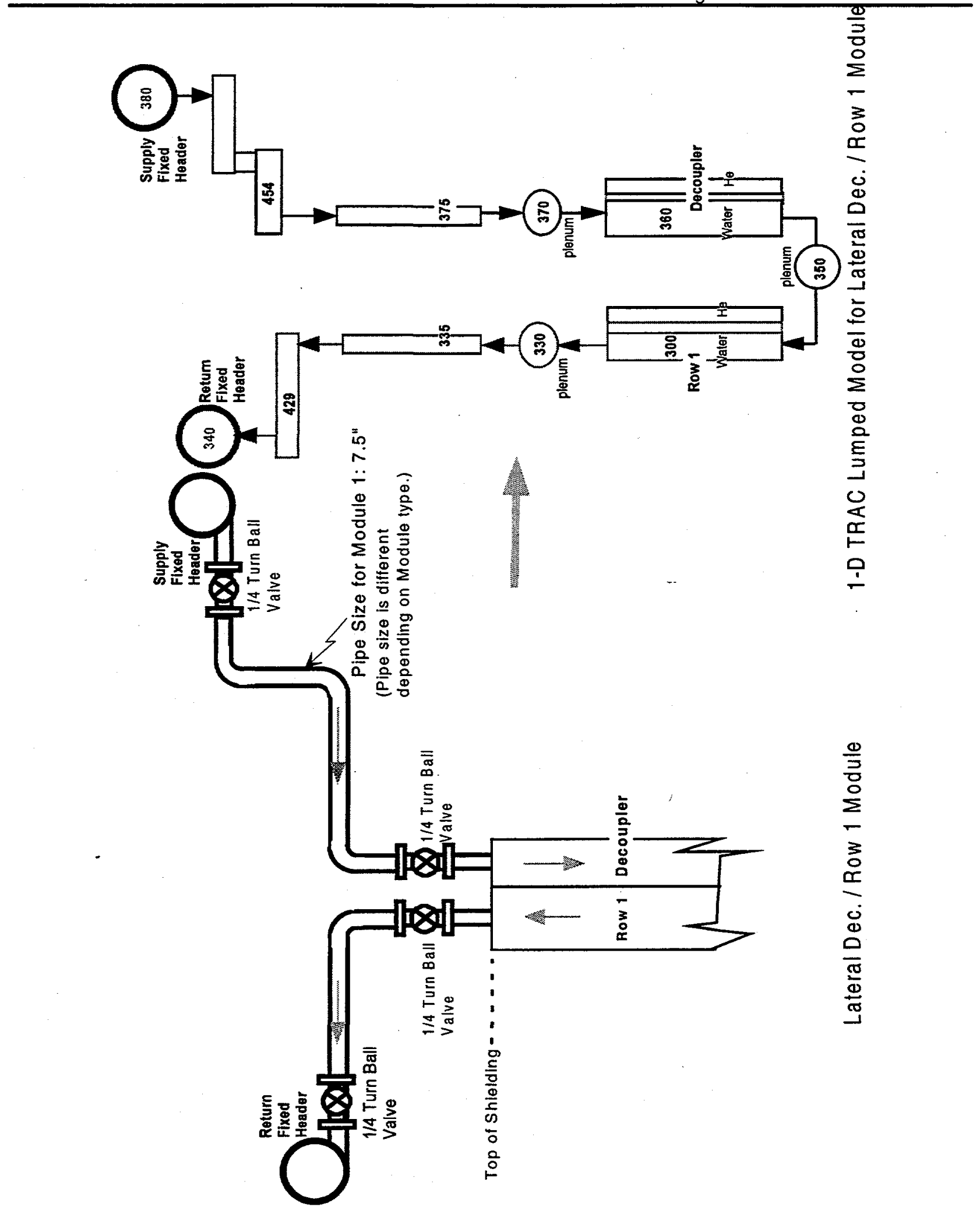

Figure 2.4-1 1-D TRAC model for Lateral Decoupler / Row 1 Module. 


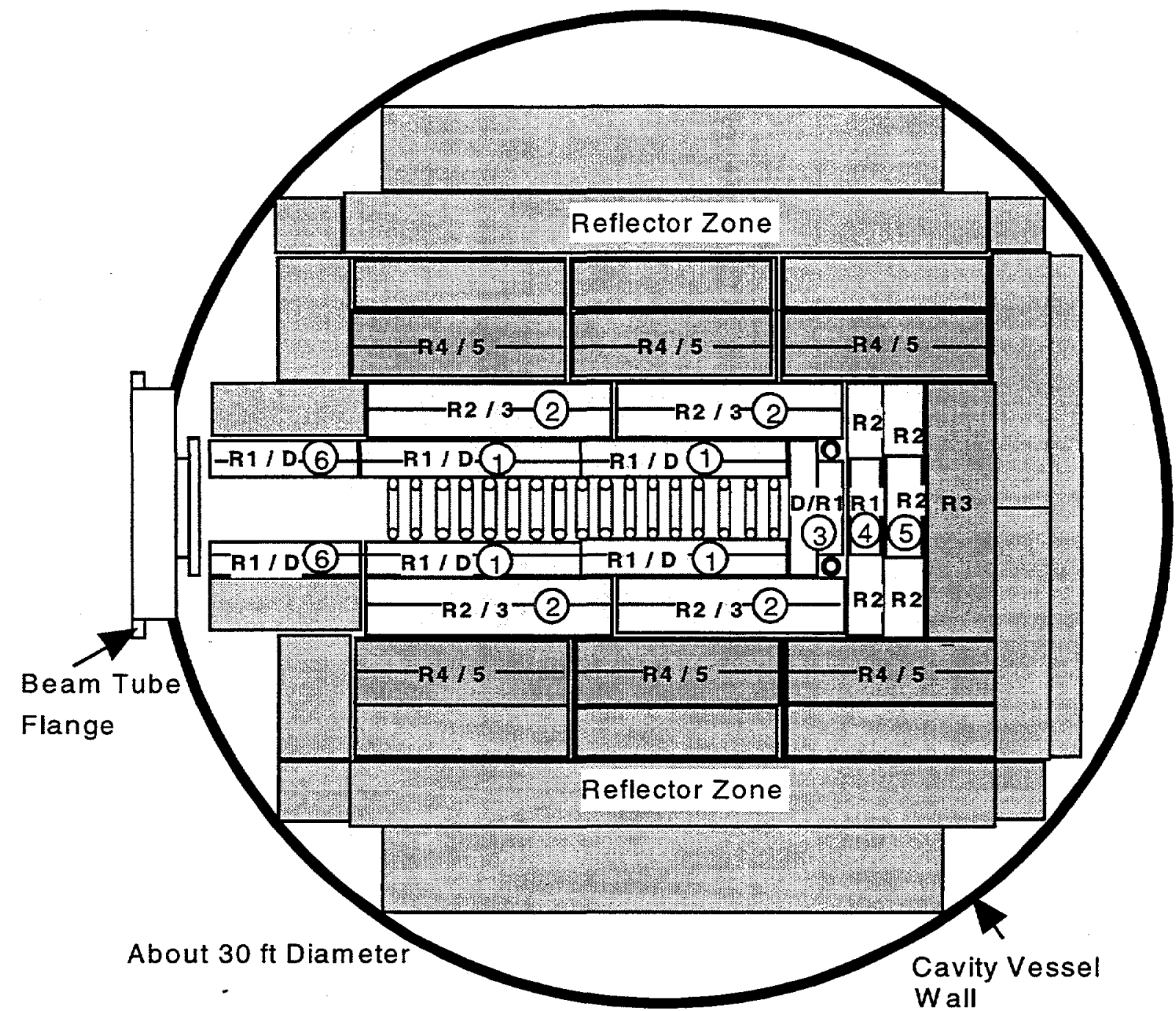

(6) Snout, 4 Bottom Modules, and 2 Top Modules

Figure 2.4-2 Top cross-sectional facemap of 6 lumped blanket system modules. 


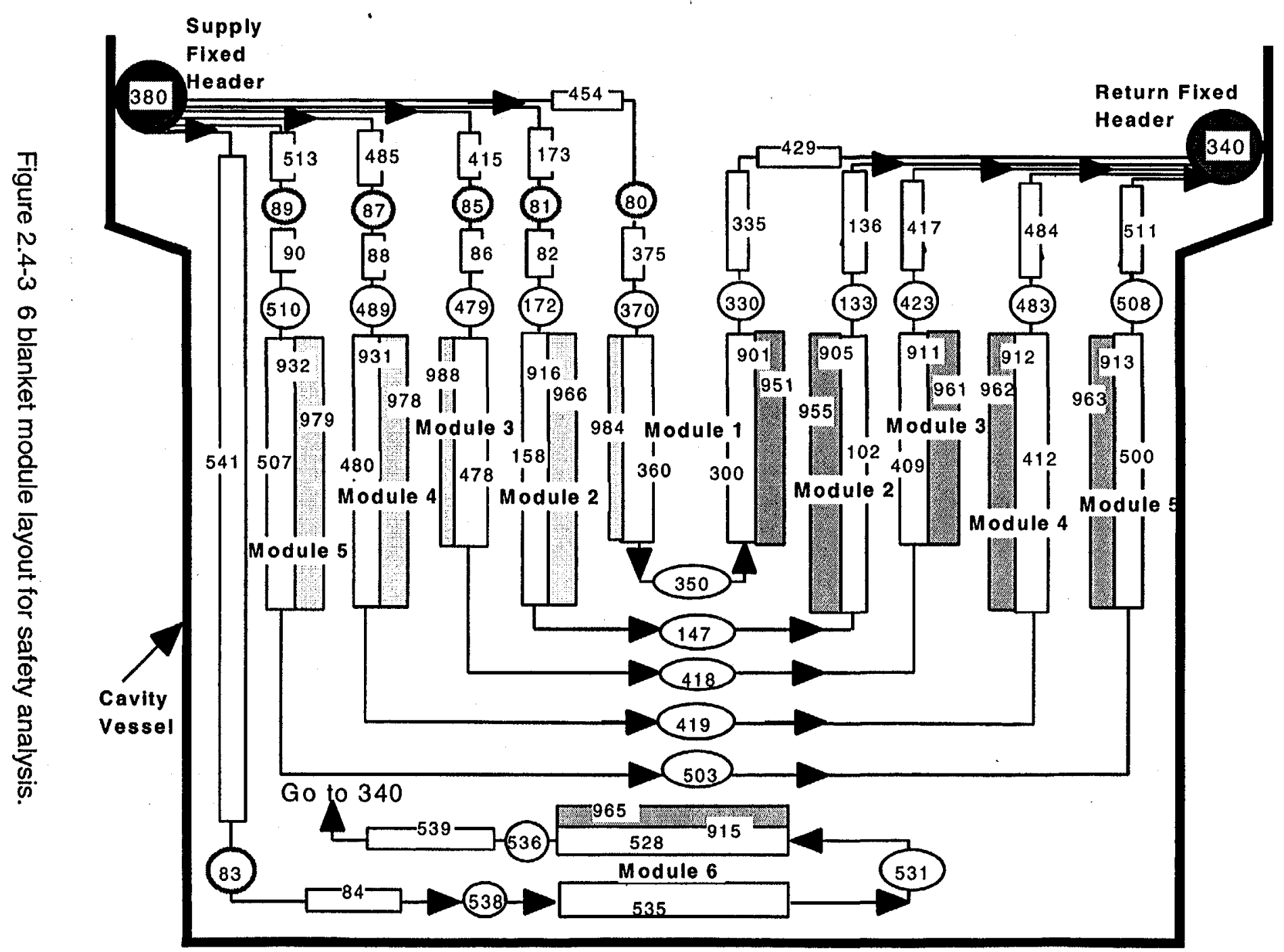

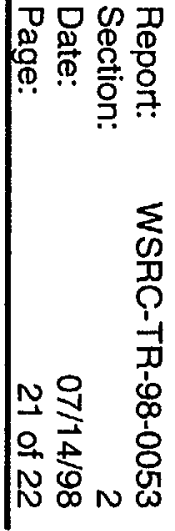




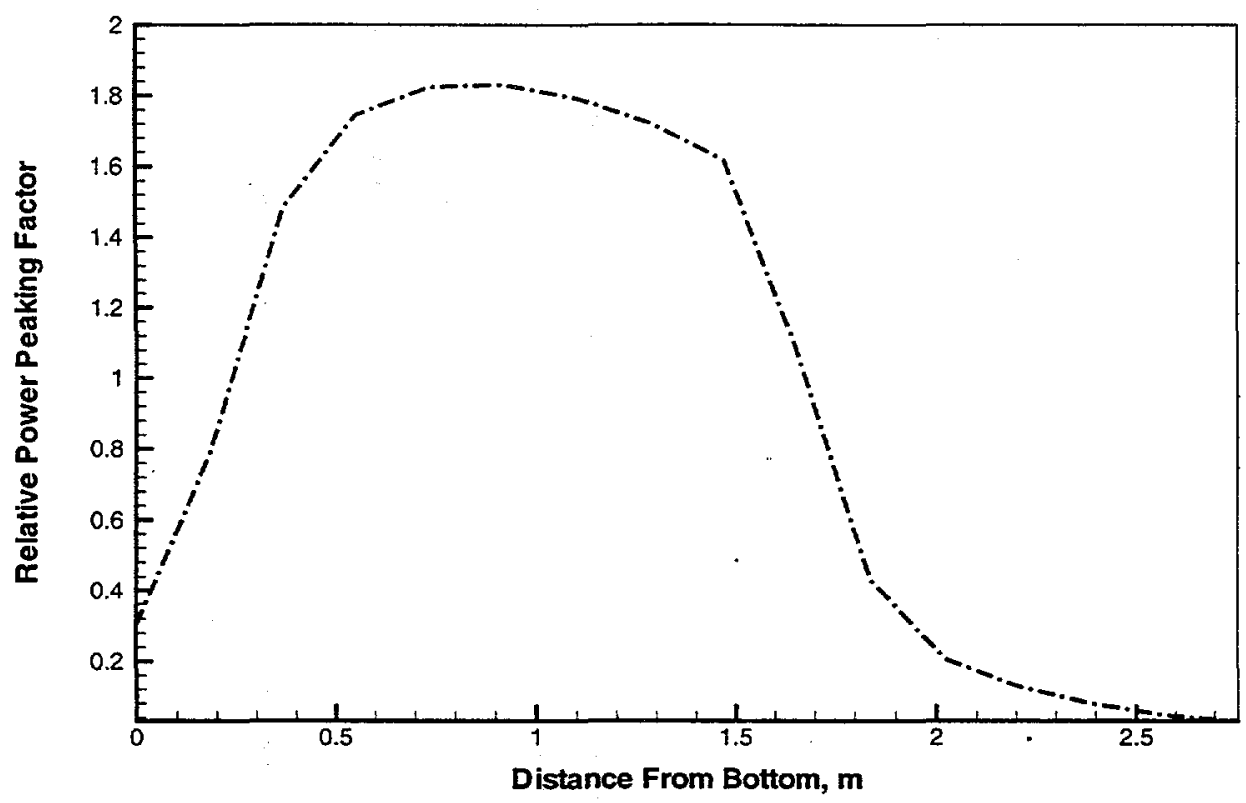

Figure 2.4-4 Typical axial power distribution for Row 1 module.

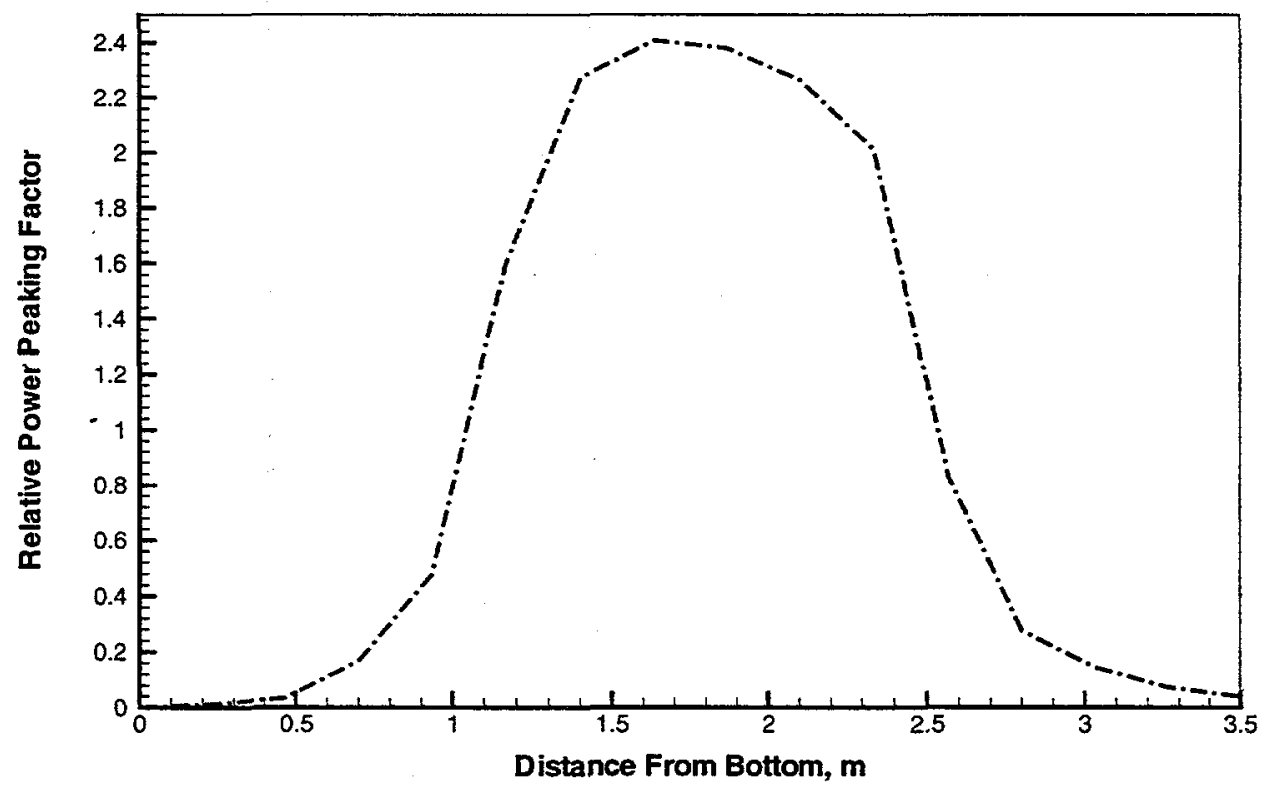

Figure 2.4-5 Typical axial power distribution for Row 2 module. 


\section{$3 \quad \mathrm{HR}$ and Component Descriptions}

\subsection{Introduction}

The purpose of the primary heat removal system is to dissipate energy that is generated within the lead blanket during normal operation. The external part of the system is the piping and components of the system, between the fixed headers, that are external to the target/blanket cavity vessel. This section of the system includes the pumps and heat exchangers. This section of the document describes the layout, which was used to develop the TRAC system model, of the external part of the primary heat removal system and supporting analyses that were instrumental in the development of the TRAC model.

\subsection{Pipe Network Layout}

Figure 3.2-1 is a schematic of the external portion of the primary heat removal system. It shows the elbows and valves in the pipe network, the pumps and heat exchangers, and the locations where the pipe diameter is reduced or enlarged. The flow proceeds from the fixed outlet header in the cavity vessel, through the pumps and heat exchangers, and back to the fixed inlet header. There are cutout valves on the suction and discharge sides of each pump and on the discharge side of each heat exchanger. There are also check valves on the discharge sides of each pump. The pipe system consists of 20 inch schedule 40 pipe for sections of the system through which the full flow travels, and 16 inch schedule 40 pipe for sections where the flow is split between the two pumps and heat exchangers. The primary heat removal system is designed to have three parallel pumps, two of which will be operating at any point in time. The non-operating pump is not included in the TRAC model, and is therefore not shown in this layout.

Figure 3.2-2 is an areal view of the primary heat removal system. This drawing is to scale, and it shows the walls that form the room in which the pumps and heat exchangers will be placed. The lengths of straight pipe are shown in brackets.

Figure 3.2-3 is a side view of the external portion of the blanket primary heat removal system. It shows the elevations of the horizontal sections of pipe. The datum for the elevations is the top of the shield in the target/blanket cavity. This is the reference elevation for the TRAC model. The four fixed headers are respectively from the lowest; the blanket outlet header, the blanket inlet header, the tungsten target outlet header, and the target inlet header. The blanket primary heat removal piping, from both the inlet and outlet headers, runs radially inward from fixed headers, goes vertically upward to the elevation $2 \mathrm{~m}$. above the centerline of the top fixed header, and then radially and horizontally out through the wall of the target/blanket cavity vessel. The elevation at which the pipes exit the cavity vessel is necessary for the pipes to clear components internal to the vessel. Once outside the cavity vessel, the pipes drop to the elevation $0.75 \mathrm{~m}$. above the floor on which the pumps and heat exchangers are supported. Lengths of straight pipe are also shown in brackets in this figure.

\subsection{Heat Exchanger Design}

Figure 3.3-1 is a schematic of a heat exchanger. It is a shell-and-tube heat exchanger, two shell and two tube passes. The blanket heat removal flow is on the tube side. The heat exchanger parameters are based on the parameters in the APT Conceptual Design Report (CDR)[1], appropriately scaled for a flowrate of $784.5 \mathrm{~kg} / \mathrm{s}$ instead of the CDR value of $477 \mathrm{~kg} / \mathrm{s}$ for each heat exchanger. The dimensions and the thermal performance of a single tube are assumed to 
remain unchanged. Therefore the number of tubes in the updated design is proportional to the ratio of new to old mass flowrates. The total number of tubes is increased from 8,400 to 13,816 , 6,908 in each pass. The ratio of the total tube flow area to the tube sheet area is assumed to also remain constant, therefore the shell diameter increases from $2.24 \mathrm{~m}$. in the CDR design to $2.87 \mathrm{~m}$. in the updated design. This preserves the heat transfer area to surrounding volume ratio on the secondary side, and justifies the assumption that the tube thermal performance remains invariant. The headers at either end of the heat exchanger are hemispherical. There is a horizontal baffle that separates the inlet and outlet headers. The assumed elevation drop, in the lumped TRAC model, between an average first pass tube and an average second pass tube is half of the shell diameter.

\subsection{Pump Head Curve}

The pumps head curve is based on the Loss of Flow Test Facility (LOFT) homologous head curve, normalized with scaled values of the pump speed, flowrate, and total dynamic head of a SRS production reactor Bingham pump [2]. The head curve is normalized with Bingham pump characteristics because reliable required net positive suction head (NPSH) data exists for these pumps, and the normalized homologous LOFT and Bingham head curves are similar. The normalized homologous head curve has the form of equation (3.4-1). The total dynamic head $(\mathrm{H})$, the pump flowrate $(\mathrm{Q})$, and the pump speed $(\mathrm{O})$ are normalized with the Bingham pump characteristics at half capacity and at the best efficiency point (BEP). The reference state is:

$$
\begin{array}{ll}
\text { pump speed } & 500 \mathrm{rpm} \\
\text { volumetric flowrate } & 12.5 \mathrm{kgpm}(0.788635 \mathrm{~m} 3 / \mathrm{s}) \\
\text { total dynamic head } & 131.2 \mathrm{ft}(40.0 \mathrm{~m})
\end{array}
$$

These are the starred symbols in equation (3.4.1).

$$
\frac{H / H^{*}}{\left(\Omega / \Omega^{*}\right)^{2}}=f\left(\frac{Q / Q^{*}}{\Omega / \Omega^{*}}\right)
$$

Figure 3.4-1 is the first quadrant of the LOFT pump head curve, along with the Bingham pump head curve. The head curves are in homologous form, and they are normalized with the reference state parameters. The LOFT head curve is defined by three points, and TRAC uses linear interpolation between them.

\subsection{Steady-State Hydraulic Model}

A simple steady-state hydraulic model of the external portion of the blanket primary heat removal system was developed to determine the necessary pipe sizes, and to compare TRAC model results with a model that includes single-phase literature based form losses. The model QKTRAC applies the mechanical energy equation to five sections of the network sequentially: from downstream of the heat exchanger discharge combining Tee to the fixed inlet header, across the parallel heat exchangers, the single pipe between the pumps and heat exchangers, across the parallel pumps, and from the fixed outlet header to upstream of the pump suction dividing Tee. The independent variables in this model are: the total volumetric flowrate, the pump speeds, and the inlet header pressure, and the model calculates pressures at the outlet header and desired intermediate locations. Since the portion of the loop between the fixed headers and inside of the cavity that contains the modules is not modeled, the system demand curve is indeterminate. At a system flowrate of $1569 \mathrm{~kg} / \mathrm{s}$, the pressure drop between the inlet and outlet headers is assumed to be approximately 40 psid. The model input pump speed is 
TRAC BLANKET SYSTEM MODEL

varied until the header-to-header pressure drop is calculated to be this value. The model QKTRAC is a fortran code that is run on a SGI work station. The code is very fast, so the user can update the pump speeds in a iterative manner between runs until the header pressure drop converges to the desired value. A code listing and also input and output files, are included in Appendix $\mathrm{C}$.

The entrance form loss for the flow from the fixed outlet header to the $20 \mathrm{in}$. pipe is treated as an abrupt contraction. Figure 3.5-1 is a schematic of the header/pipe connection, showing the flow areas, and it also shows that the contraction upstream flow area is assumed to be twice the header flow area. Equation 3.5.1 is the expression for the form loss coefficient for an abrupt contraction [3]. The pressure drop is based on the higher velocity. The exit form loss for the flow from the pipe into the fixed inlet header is treated as an abrupt expansion, and the equivalent header flow area is treated in the same manner as for the inlet header. Equation 3.5.2 is the expression for the form loss coefficient, and it is again based on the higher velocity.

$$
\begin{gathered}
K=0.42\left[1-\left(\frac{D_{2}}{D_{1}}\right)^{2}\right] \\
K=\left(1-\frac{A_{1}}{A_{2}}\right)^{2}
\end{gathered}
$$

These expressions are used to calculate the expansion and contraction form loss coefficients for the pipe diameter transitions and the flows into and out of the heat.exchanger headers. The loss coefficients are:

- Contraction from fixed outlet header into 20 " pipe

- Expansion from 20" pipe to fixed inlet header

$K=0.243$

- Contraction from $20^{\prime \prime}$ to $16^{\prime \prime}$ pipe

$K=0.335$

$\mathrm{K}=0.153$

- Expansion from 16" to 20" pipe

- Expansion from 16" pipe into heat exchanger inlet header

$K=0.133$

- Contraction from header into heat exchanger tubes

- Expansion heat exchanger tubes into header

$K=0.931$

- Contraction from heat exchanger header into 16 " pipe

$K=0.347$

$K=0.681$

$K=0.405$

The 90 degree elbow form loss coefficients are calculated from the fully-developed turbulent frictional pressure drop in an equivalent length of straight pipe [4]. The elbow form loss coefficients are:

$$
\begin{aligned}
& K_{20^{n}}=0.156 \\
& K_{16^{\prime \prime}}=0.159
\end{aligned}
$$

The form loss coefficients for fully open gate valves are 0.047 and 0.5 for fully open check valves [5].

These form loss coefficients are used in QKTRAC and, where appropriate, in the primary heat removal system TRAC model.

\subsection{Pipe Size Design Study}

QKTRAC was used to size the pipes in the flow network, with the criteria that the pumps not cavitate under normal operating conditions. Equation 3.6.1 is the expression for the required 
NPSH [6] in meters. This expression has been scaled to the half speed reference state listed in sub-section 3.4 .

$$
N P S H_{R}=2.256\left(\frac{\Omega}{\Omega^{*}}\right)^{2}+5.334\left(\frac{Q}{Q^{*}}\right)^{2}
$$

Four different combinations of main pipe diameters and smaller diameters of the secondary pipes associated with the individual pumps and heat exchangers were considered, and the results of the model are presented Table 3.6-1. All diameters are in inches and are nominal values for schedule 40 steel pipes. Shown are the available and required NPSH values for pumps one and two respectively. Cavitation is predicted if the available NPSH is less than the required. Main pipes with diameters less than eighteen inches are unsuitable. Eighteen inch main and fourteen inch secondary pipes meet the criteria. The design authority at Los Alamos National Laboratory suggested twenty inch main and sixteen inch secondary piping, to ensure plenty of margin. Pipe is relatively inexpensive, and a larger pipe diameter could lower pump operating costs.

\subsection{TRAC Modeling of Tee's}

As part of the verification process of the TRAC model of the external portion of the primary heat removal system, results of a stand-alone TRAC model were compared with the results of the simple hydraulic model. In the course of this study, it was ascertained that the TRAC TEE component significantly over-predicted the irreversible pressure drop across the legs of the Tee. Figure 3.7-1 shows schematics of symmetric and asymmetric Tee's for both dividing and combining flows, along with branch nomenclature for the form loss coefficient correlations. These are the four types of Tee's that occur in the network. Equation 3.7.1 is the correlation for the form loss in a dividing symmetric Tee [5]. The pressure drop is based on the incoming velocity.

$$
K_{12}=0.59+\left[1.18-1.84\left(\frac{r}{D}\right)^{1 / 2}+1.16\left(\frac{r}{D}\right)\right]\left(\frac{U_{2}}{U_{1}}\right)-\left[0.68-1.04\left(\frac{r}{D}\right)^{1 / 2}+1.16\left(\frac{r}{D}\right)\right]\left(\frac{U_{2}}{U_{1}}\right)^{2}
$$

Figure 3.7-2 shows the pressure drops across a symmetric dividing Tee, as functions of the inlet mass flowrate, predicted by TRAC and from literature loss coefficients, equation 3.7.1. A horizontal Tee with 16 inch schedule 40 pipe and an equal flow split between the two discharge legs is assumed. The Tee junction is assumed to not be rounded, $r / D=0$. TRAC predicts pressure drops that are approximately four times those from literature correlations. To correct this discrepancy, Tee's in the TRAC model were replaced by PLENUM components, and literature based form loss coefficients are used. Equation 3.7.2 is the correlation for the form loss in a combining symmetric Tee [5]. The pressure drops are based on the outgoing velocity.

$$
K_{13}=1.19-1.16\left(\frac{r}{D}\right)^{1 / 2}+0.46\left(\frac{r}{D}\right)-1.73\left(1-\frac{r}{D}\right) \frac{U_{2}}{U_{3}}+\left(1.34-1.69 \frac{r}{D}\right)\left(\frac{U_{2}}{U_{3}}\right)^{2}
$$

Equations 3.7.3 and 3.7.4 are correlations for form loss coefficients for an asymmetric combining Tee [5]. The pressure drops are again based on the outgoing velocity.

$$
K_{13}=0.045+\left[1.38-1.94\left(\frac{r}{D}\right)^{1 / 2}+1.34 \frac{r}{D}\right] \frac{U_{2}}{U_{3}}-\left[0.90-0.95\left(\frac{r}{D}\right)^{1 / 2}+1.23 \frac{r}{D}\right]\left(\frac{U_{2}}{U_{3}}\right)^{2}
$$




$$
K_{23}=1.09-0.80\left(\frac{r}{D}\right)^{1 / 2}-\left[0.53+1.27\left(\frac{r}{D}\right)^{1 / 2}-1.86 \frac{r}{D}\right] \frac{U_{1}}{U_{3}}-\left[1.48-2.28\left(\frac{r}{D}\right)^{1 / 2}+1.80 \frac{r}{D}\right]\left(\frac{U_{1}}{U_{3}}\right)^{2}
$$

Equations 3.7.5 and 3.7.6 are correlations for form loss coefficients for an asymmetric dividing Tee [5]. The pressure drops are based on the incoming velocity.

$$
\begin{gathered}
K_{12}=0.65\left(\frac{U_{3}}{U_{1}}-0.22\right)^{2}-0.03 \\
K_{13}=0.99-0.23\left(\frac{r}{D}\right)^{1 / 2}-\left[0.82+0.29\left(\frac{r}{D}\right)^{1 / 2}+0.30 \frac{r}{D}\right] \frac{U_{3}}{U_{1}}+\left[1.02-0.64\left(\frac{r}{D}\right)^{1 / 2}+0.76 \frac{r}{D}\right]\left(\frac{U_{3}}{U_{1}}\right)^{2}
\end{gathered}
$$

Figs. 3.7-3 through 3.7-6 show comparisons between TRAC with PLENUM components in place of TEE components and literature based pressure drops for the four Tee configurations. The kinetic energy terms are included in the literature based pressure drops, and these account for the large differences in pressure drops between combining and dividing Tee's. There is actually a pressure recovery in the straight pipe section of an asymmetric dividing Tee. The irreversible pressure drops though are always positive. In all cases, the pressure drops calculated with the literature correlations are less than the TRAC results because friction is neglected in the literature based results. The agreement is otherwise excellent. In all cases, equal branch flowrates were assumed, and the pipe junctions are assumed to be square, $r / D=0$. TRAC requires that the loss coefficients be constants, whereas the correlations are functions of the flow splits. Ideally the flows through the parallel pumps and heat exchangers should be equal, and would be if the branch piping layouts were symmetric. They are not, but the branch flowrates are close to equal, and it is a reasonable assumption in calculating the Tee loss coefficients to assume that they are, because Tee pressure drops are small in comparison with the overall pressure drops across the pump and heat exchanger branches.

\subsection{TRAC Model Steady-State Benchmarking}

Once the TEE components were replaced in the TRAC model, the agreement between the TRAC model and the simple hydraulic model was excellent, Table 3.8-1. The pressures are high because the TRAC model was run at a high system pressure, during the development process, to ensure that flashing did not occur. This comparison was made with a TRAC model of an earlier primary heat removal system pipe layout. The requirement that the pipes exit the cavity vessel two meters above the highest fixed header had not been promulgated when this comparison was made. Minor changes in pipe lengths and elevations should not change the close agreement between TRAC and the hydraulic model QKTRAC. This comparison will be repeated for future pipe network design layouts, as time permits.

\subsection{TRAC Model Component Layout}

Figure 3.9-1 shows the TRAC component layout and junction numbers for the external portion of the blanket primary heat removal system. Also shown is the component layout of the blanket primary pressurizer. A brief description of and the number of cells in each component of the entire TRAC model is listed in Table 2.4-2. Further details such as cell lengths, flow areas, hydraulic diameters, and form loss coefficients can be found in the TRAC model input deck (see Appendix A). 
The components modeling the lumped first and second pass heat exchanger tubes are PIPE components 50 and 54 respectively for heat exchanger number one and 51 and 55 for heat exchanger number two. HEAT STRUCTURES and secondary side flow components for the heat exchangers are also shown in Fig. 3.9-1, the 700 series components. Components 30 and 31 are the two PUMP components, and VALVE components 32 and 33 model the check valves on the discharge side of each pump. PLENUM components 380 and 340 are respectively the fixed inlet and outlet headers. PIPE component 37 is the location for pump discharge large break LOCA's, and component 65 is the location for a large break LOCA external to the cavity vessel and close to the inlet header. The internal to the cavity large break LOCA is modeled by the group of components that includes CONTROL VALVE components 850, 852, and 854. Component 826 is a PIPE component in the cavity vessel. To simulate a break, VALVE 854 is shut, and VALVES 850 and 852 open in concert.

\subsection{References}

1. "APT Conceptual Design Report," Los Alamos National Laboratory report, LA-UR-97-1329 (April 1997).

2. L. L. Hamm and D. A. Crowley, "1985 L-Reactor Test Results Used to Determine PressureFlow Relationships," E. I. Du Pont de Nemours \& Co., DPST-88-814, December 1988.

3. P. M. Gerhart and R. J. Gross, Fundamentals of Fluid Mechanics, Addison Wesley Publishing Company, 1985.

4. R. W. Fox and A. T. McDonald, Introduction to Fluid Mechanics, John Wiley \& Sons, Inc., 1973.

5. R. D. Blevins, Applied Fluid Dynamics Handbook, Van Nostrand Reinhold Company, 1984.

6. L. L. Hamm, "Reanalysis of Bingham Pump Cavitation Characteristics for PLK Reactors," E. I. Du Pont de Nemours \& Co., DPST-84-624, August 1984.

Table 3.6-1 Estimated required and available NPSH for HR piping.

\begin{tabular}{|c|c|c|}
\hline $\begin{array}{c}\text { Pipe Diameters } \\
\text { (main-secondan) }\end{array}$ & NPSH Available $(\mathbf{m})$ & $\begin{array}{c}\text { NPSH Required }(\mathrm{m}) \\
\text { (pumps } / 2)\end{array}$ \\
\hline $14-10$ in. & $-16.4 /-10.8$ & $7.7 / 8.4$ \\
$16-12$ & $-1.5 / 2.9$ & $7.3 / 7.9$ \\
$18-14$ & $14.0 / 16.9$ & $7.0 / 7.4$ \\
$20-16$ & $23.9 / 26.0$ & $6.8 / 7.1$ \\
\hline
\end{tabular}

Table 3.8-1 Comparison between TRAC and simple hydraulic model pressure profile for HR piping loop.

\begin{tabular}{|c|c|c|}
\hline Location & RHRTRC pressure (psia) & TRAC pressure (psia) \\
\hline Fixed Outlet Header & 163.15 & 165.3 \\
\hline Pump Suction & 144.93 & 145.4 \\
\hline Pump Discharge & 226.19 & 225.6 \\
\hline Heat Exchanger Inlet & 224.50 & 224.6 \\
\hline Heat Exchanger Outlet & 209.86 & 209.5 \\
\hline Fixed Inlet Header & 203.20 & 203.20 \\
\hline
\end{tabular}




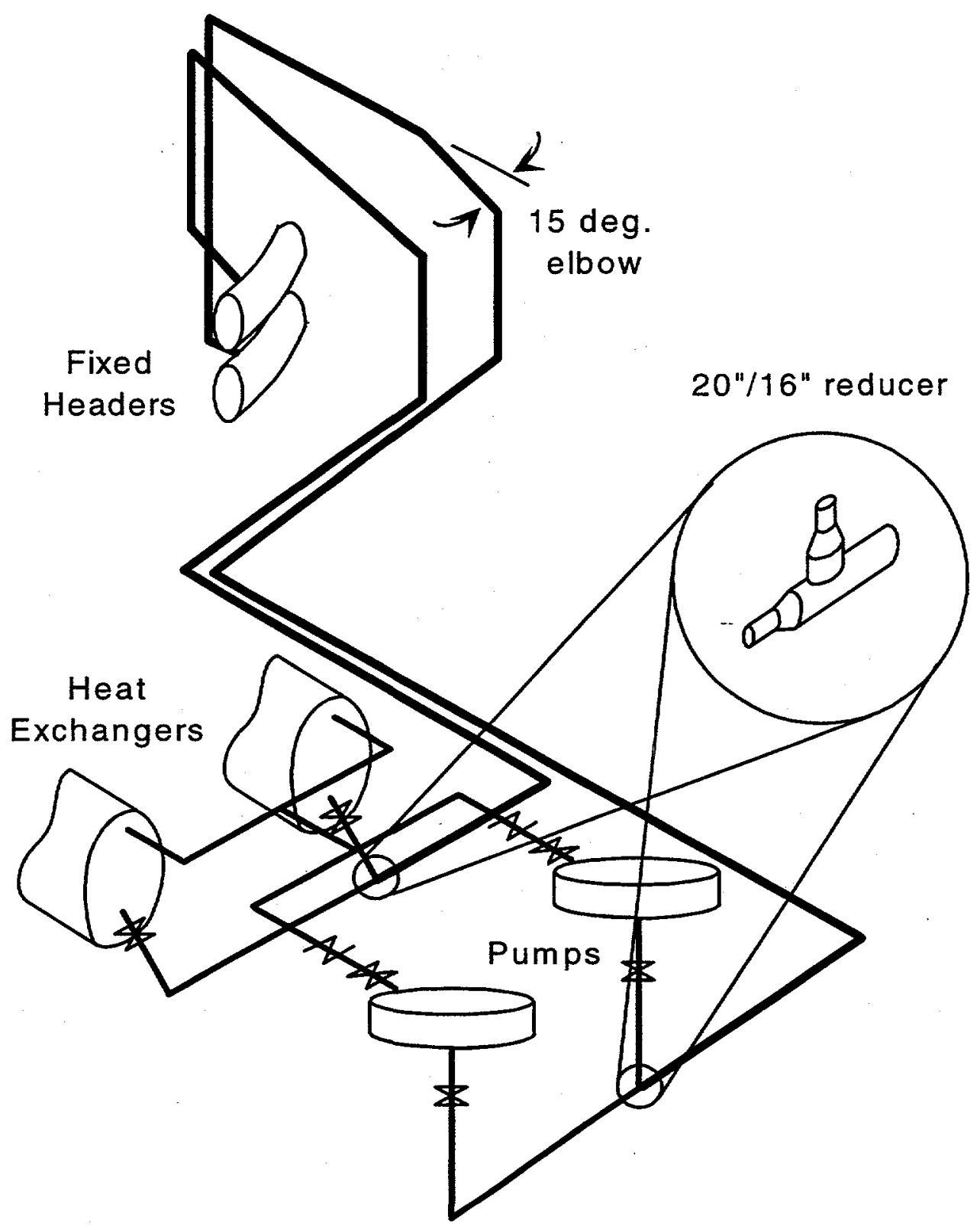

Figure 3.2-1 Schematic of the external portion of the blanket primary heat removal system. 


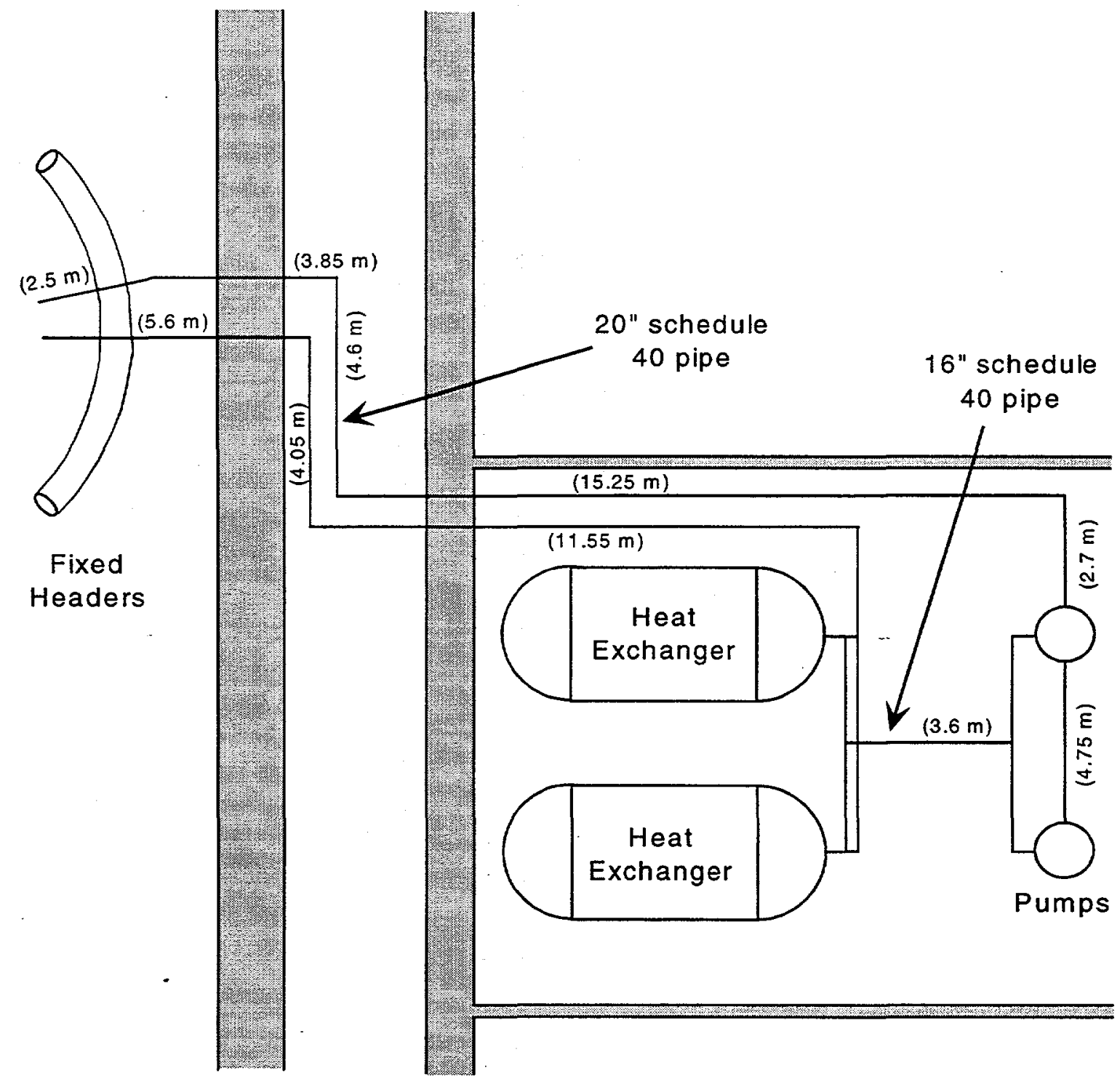

Figure 3.2-2 Areal view of the of the external portion of the blanket primary heat removal system. Lengths of straight pipe are in parentheses. 


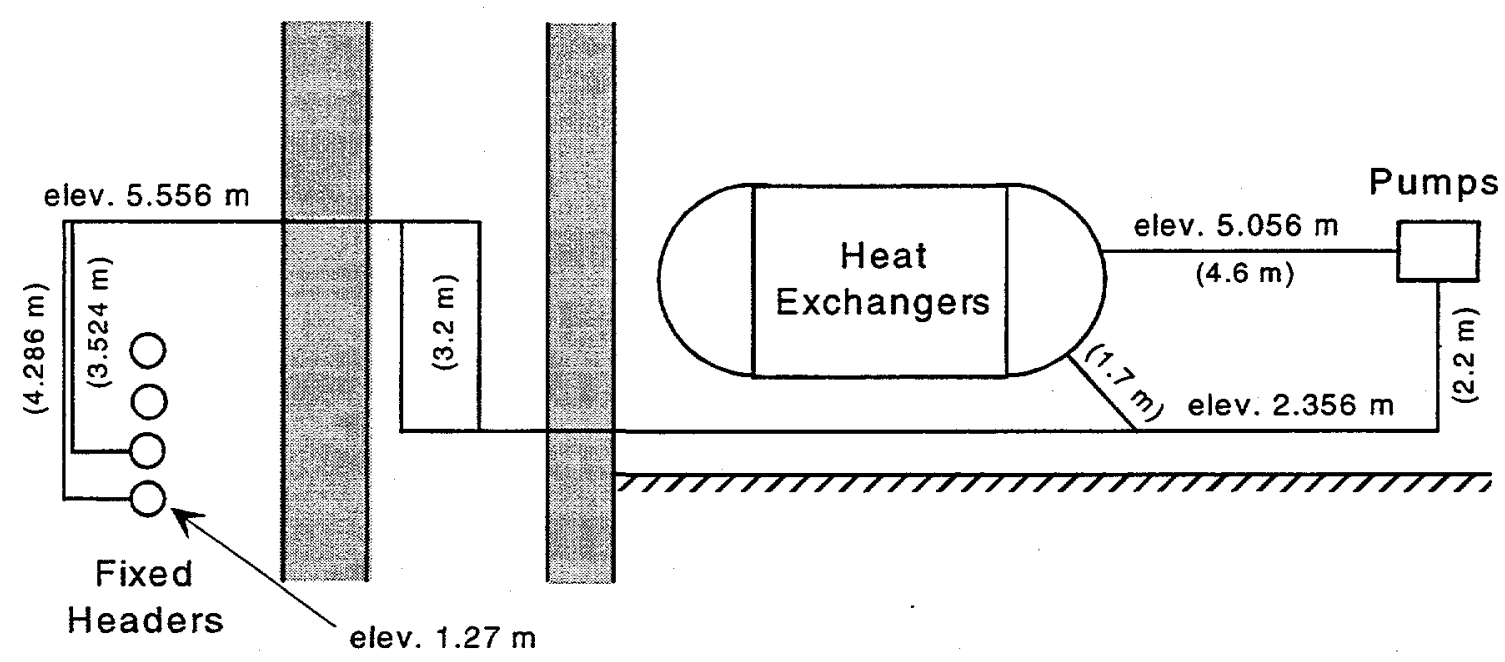

Figure 3.2-3 Side view of the external portion of the blanket primary heat removal system. Lengths of straight pipe are in brackets.

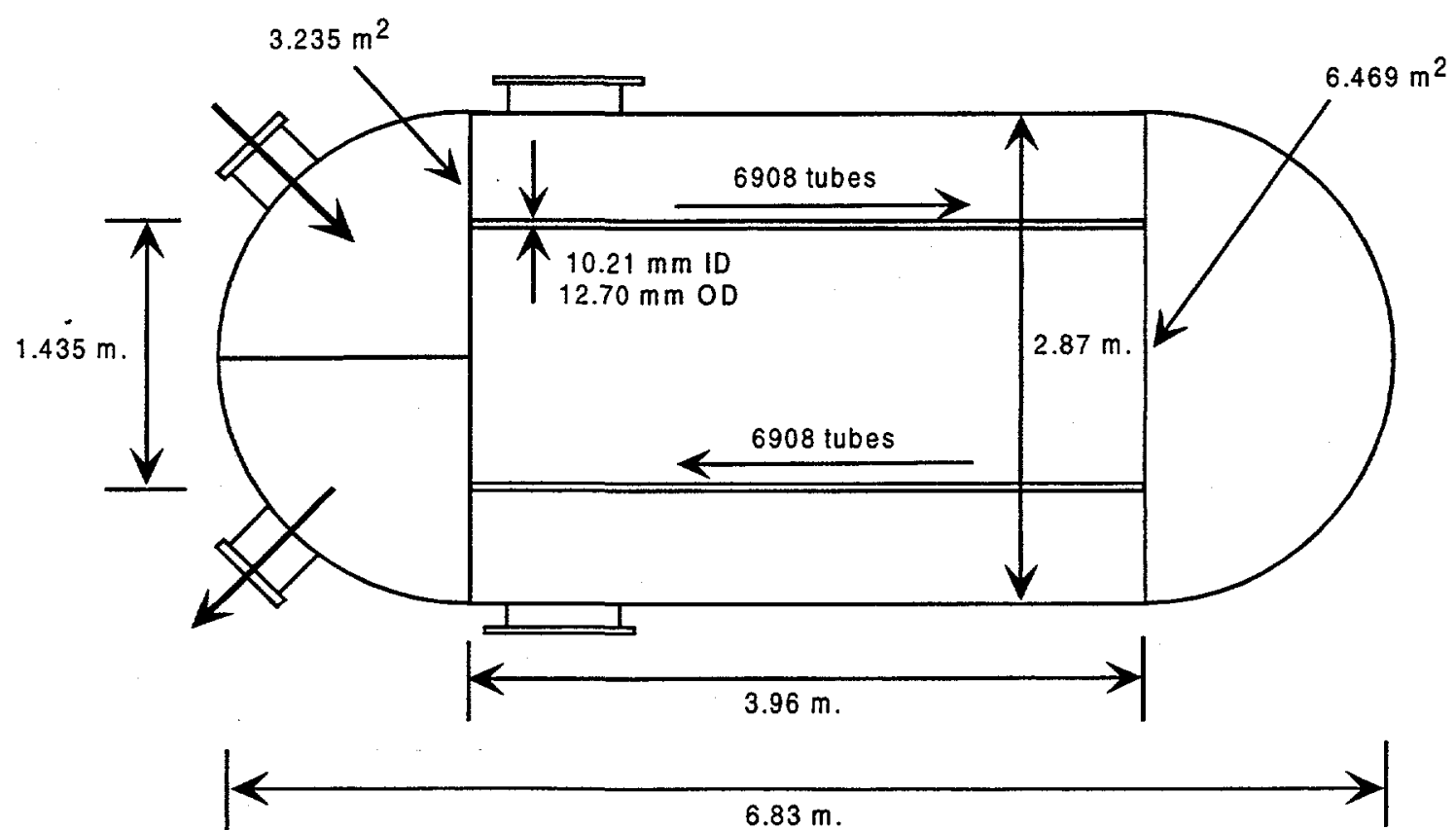

Figure 3.3-1 Schematic of a blanket primary heat removal system heat exchanger. 


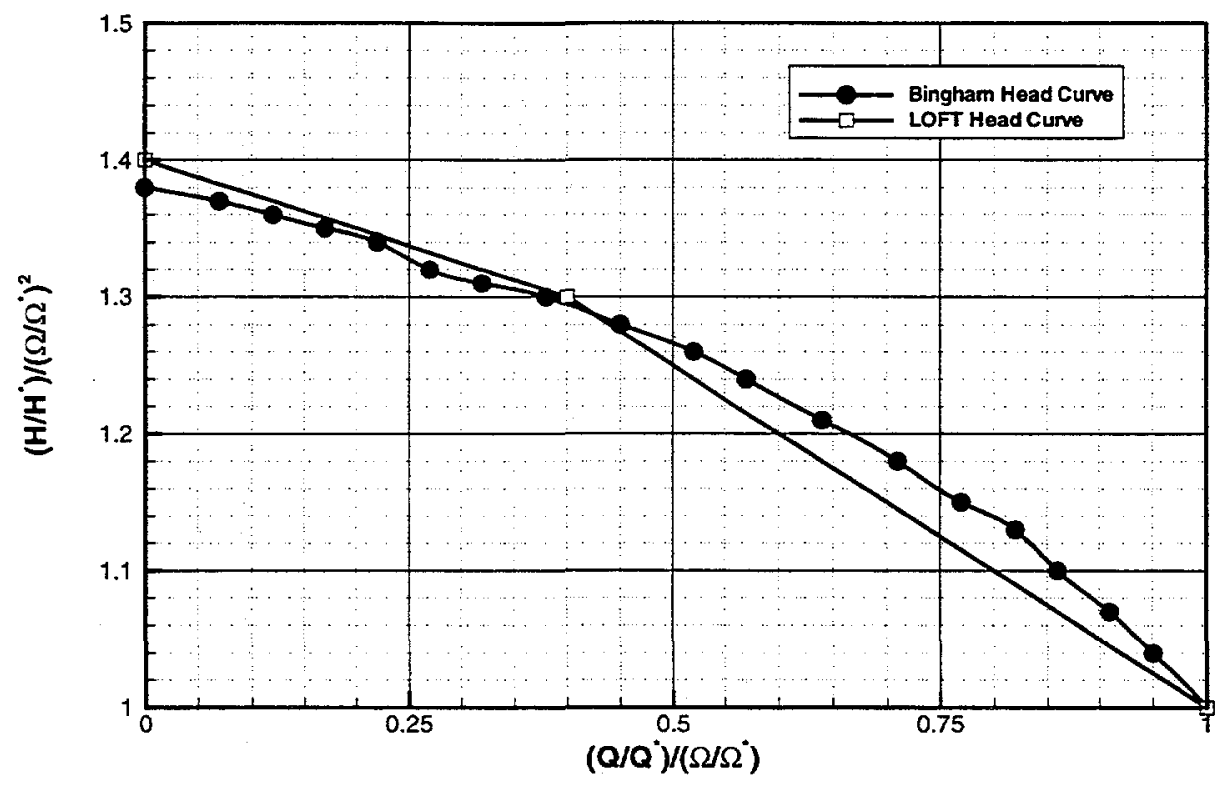

Figure 3.4-1 Pump normalized homologous head curves.

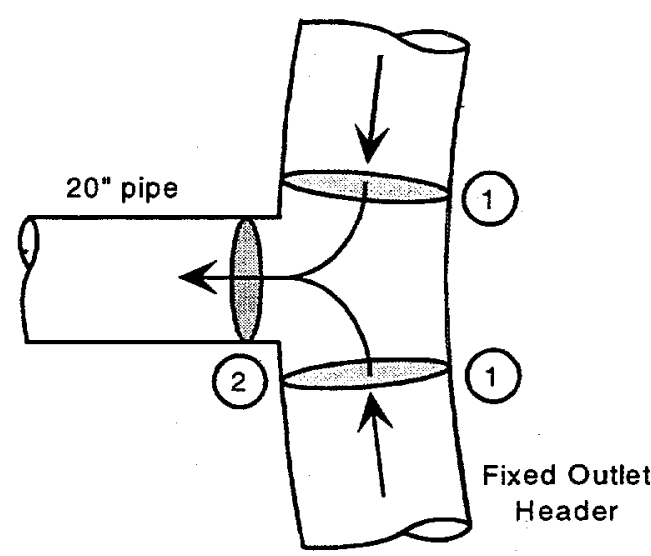

Equivalent Header

Flow Area

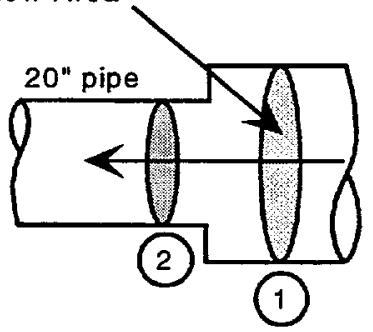

Figure 3.5-1 Schematic of the fixed header/pipe connection, showing how the header flow areas are treated to calculate an abrupt contraction form loss coefficient. 


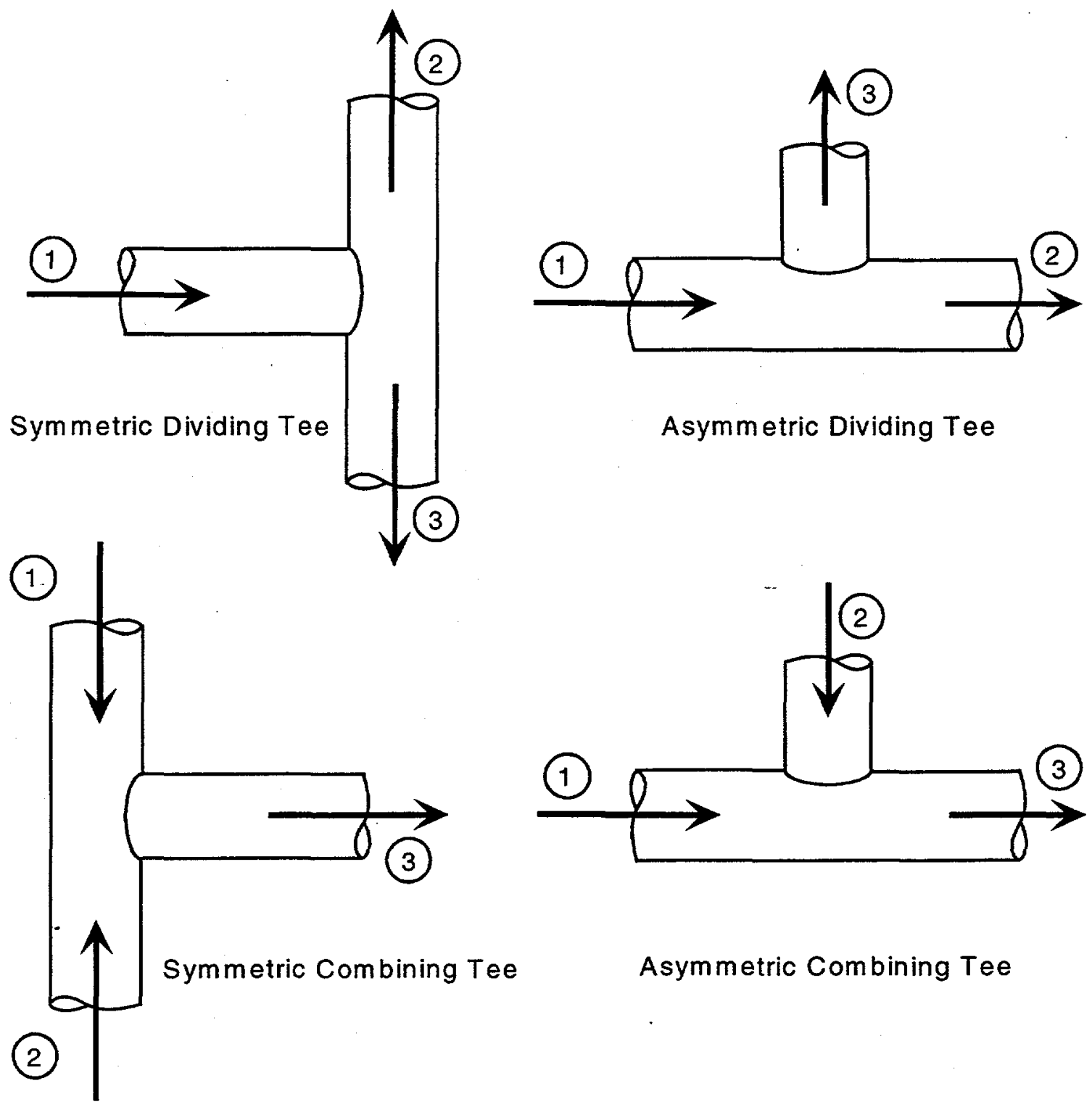

Figure 3.7-1 Schematic of dividing Tee pipe components. 


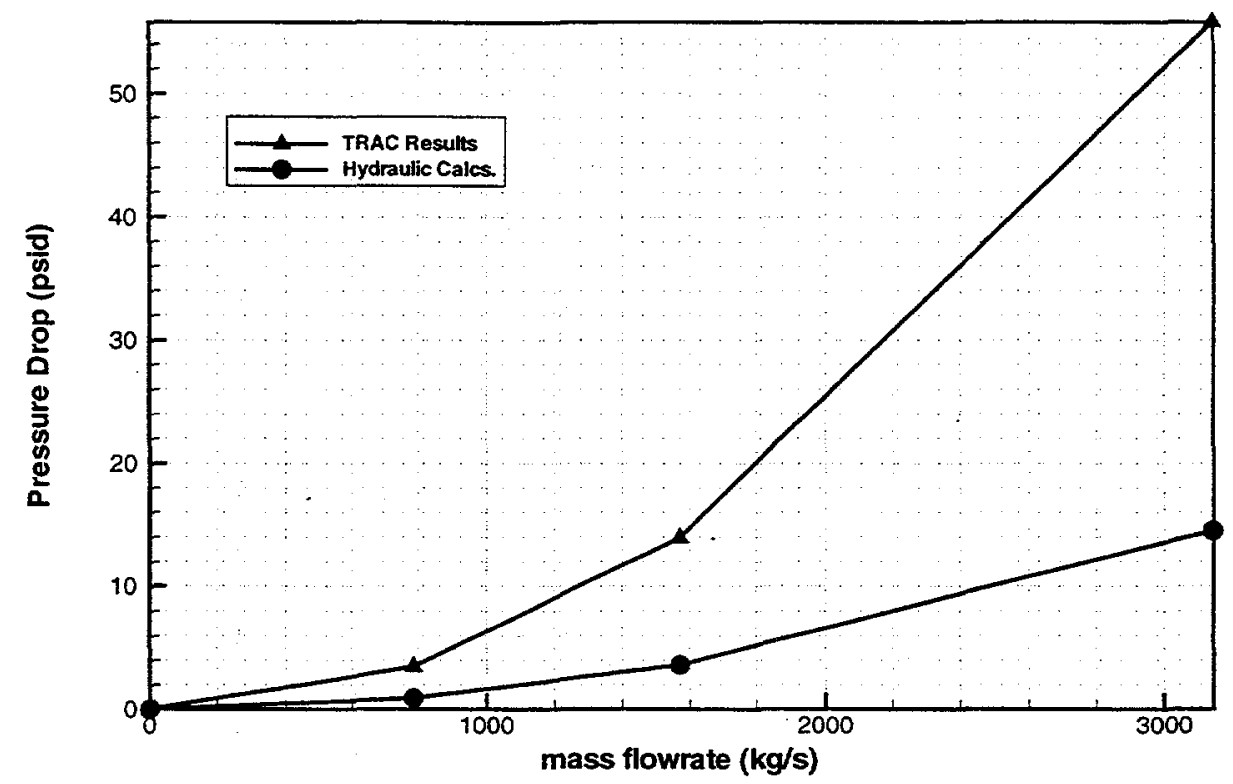

Figure 3.7-2 Pressure drop comparison across a symmetric dividing Tee, between a TRAC Tee component and literature loss coefficients.

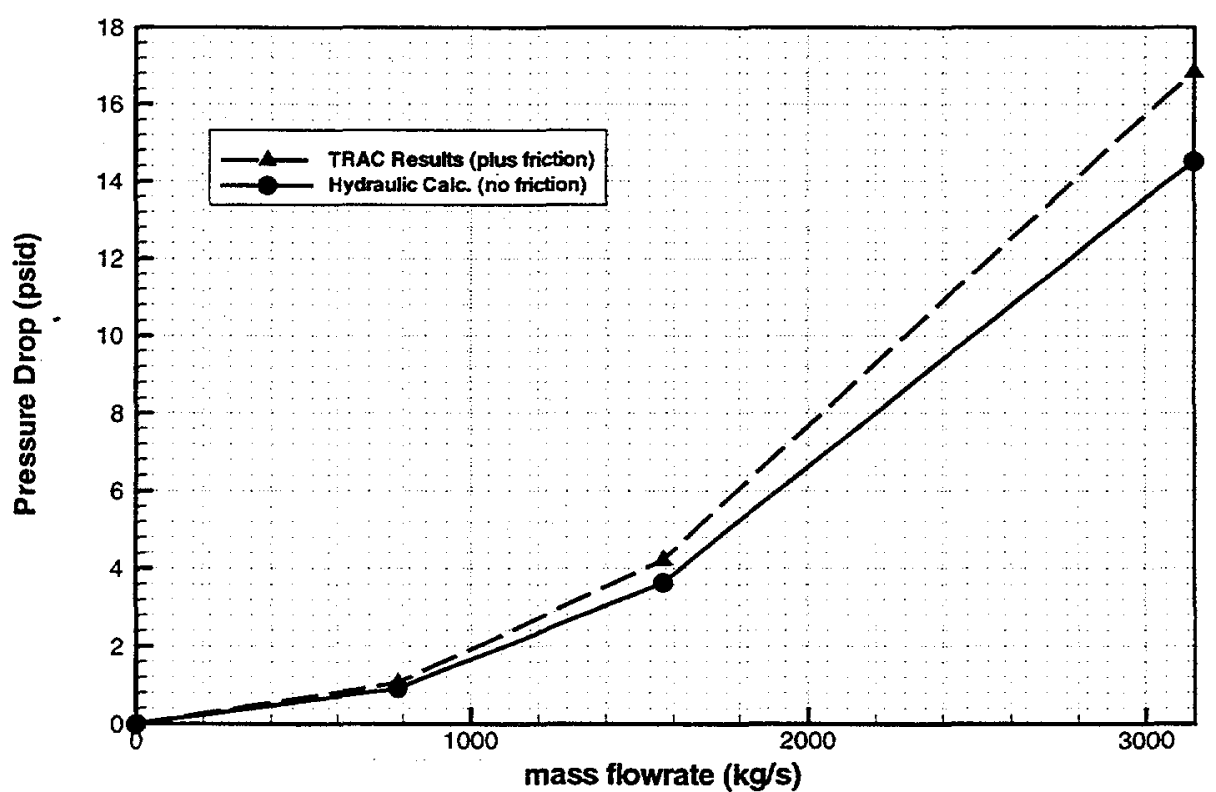

Figure 3.7-3 Pressure drop comparison across a symmetric dividing Tee, between a TRAC plenum component and literature loss coefficients. 


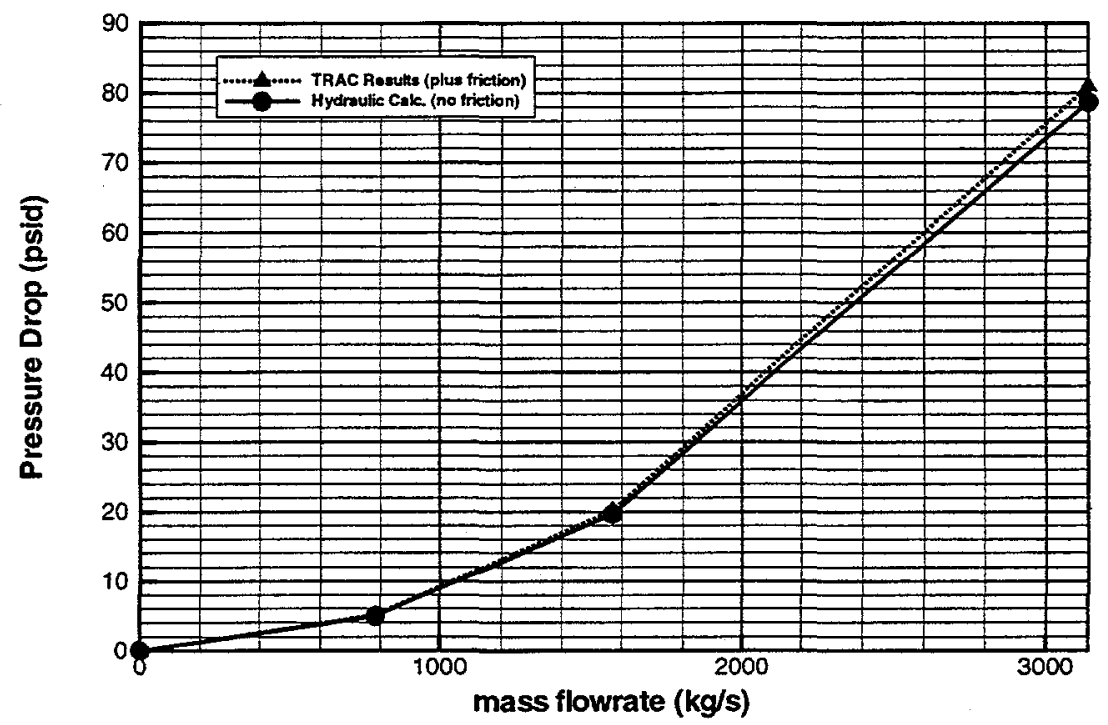

Figure 3.7-4 Pressure drop comparison across a symmetric combining Tee, between a TRAC plenum component and literature loss coefficients.

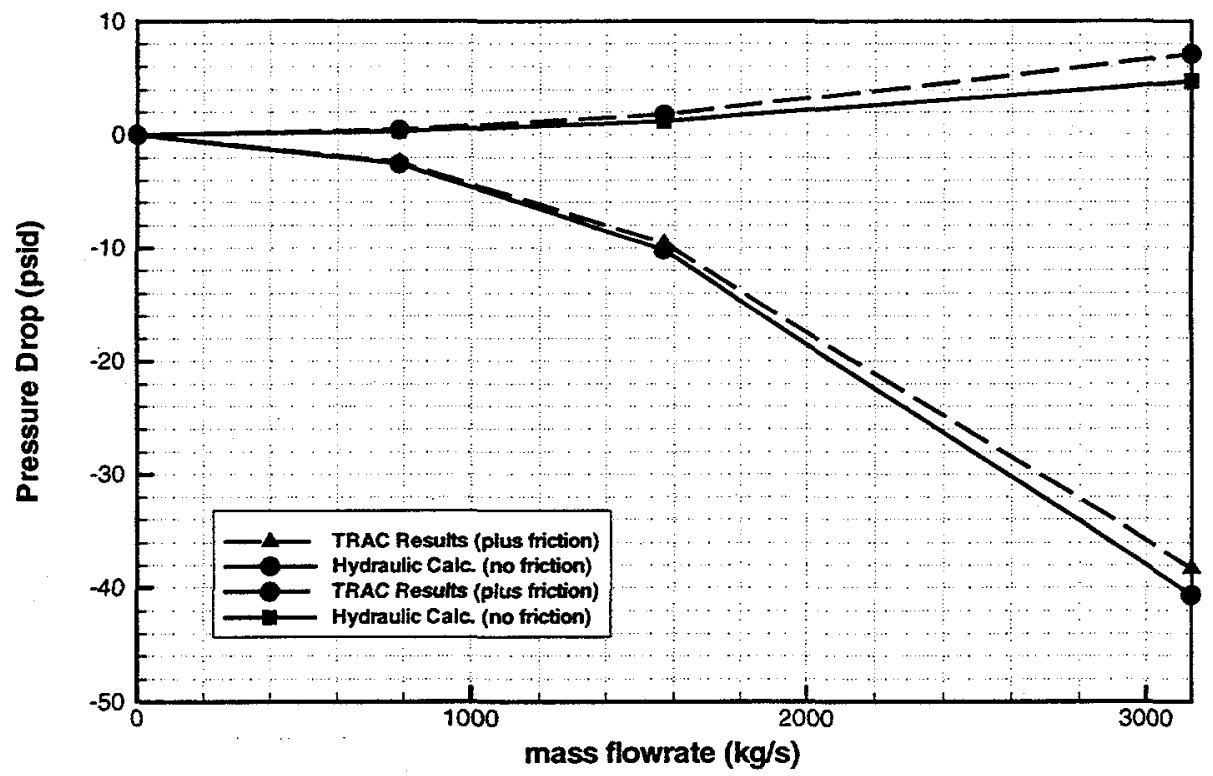

Figure 3.7-5 Pressure drop comparison across an asymmetric dividing Tee, between a TRAC plenum component and literature loss coefficients. 


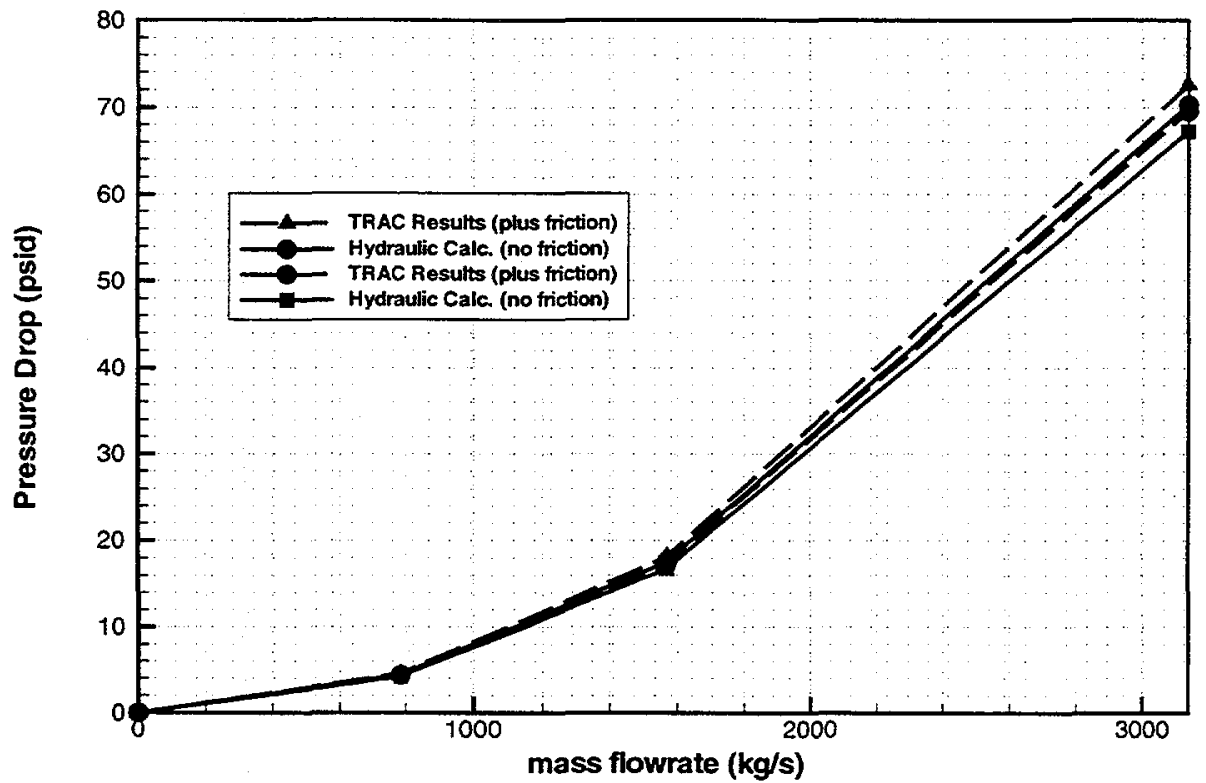

Figure 3.7-6 Pressure drop comparison across an asymmetric combining Fee, between a TRAC plenum component and literature loss coefficients. 


\section{Pressurizer Description}

\subsection{Introduction}

The blanket heat removal system is pressurized to approximately 100 psia to prevent cavitation from occurring anywhere in the system during normal operation. The pressurizer also serves as a reservoir for replacing lost coolant inventory in the event of an accident. The pressurizer is a large vessel, partially filled with water, that is connected by pipe to the fixed inlet header. There is a helium filled gas space above the water, and the gas space pressure determines the inlet header pressure. During normal operation, the gas space pressure is 97.6 psia. The pressurizer will prevent a rapid system depressurization during a LOCA.

\subsection{Pressurizer Design}

Figure 4.2-1 is a schematic of the pressurizer vessel and the surge line that connects it to the fixed inlet header. The vessel is $8.54 \mathrm{~m}$. high, and it has a diameter of $3.658 \mathrm{~m}$. Water fills the vessel to a height of $6.723 \mathrm{~m}$. The pressurizer is connected to the inlet header by a $10^{\prime \prime}$ schedule 40 steel pipe, with a length of $31.698 \mathrm{~m}$. Figure $4.2-1$ is to scale vertically, but not horizontally.

The pressurizer was designed to not completely drain during a LOCA, thereby not entraining gas in the heat removal system flow. Two additional design constraints are that the water in the vessel not flash, and cavitation not occur in the surge line during the drain down transient.

\subsection{Pressurizer Drain Down Design Study}

A stand-alone TRAC model of the pressurizer and surge line was developed and exercised parametrically, to determine the initial level of water in the vessel, the surge line diameter, and the elevation of the vessel. Figure 4.3-1 is a TRAC component layout of this model. The accident scenario chosen for this design study was a large-break LOCA, internal to the cavity, just above the top shield. The break location is assumed to be $1.532 \mathrm{~m}$. below the header. The cavity vessel has a vacuum breaker that will be actuated in the event of an internal break, consequently the cavity will be at atmospheric pressure several minutes after the occurrence of an internal LOCA, and the inlet header pressure will be 12.53 psia, based on the hydrostatic difference. This is the break component pressure boundary condition for the stand-alone TRAC model.

A final liquid depth of $0.625 \mathrm{~m}$. was chosen as the target for this design study. This results in a void fraction of 0.5 in the vessel component cell adjacent to the bottom cell. Since TRAC cannot handle multiple non-condensable constituents, the gas space was assumed to be filled with air. The initial liquid temperature was assumed to be $40 \mathrm{C}$.

Figures 4.3-2 through 4.3-8 show results of the stand-alone TRAC model. Figure 4.3-2 shows the fluid pressures in the pressurizer vessel component cells. The final pressure in the gas space, after completion of the expansion, is 12.39 psia. Figure 4.3-3 shows the fluid temperatures in the pressurizer. The liquid remains at the initial temperature of $40 \mathrm{C}$. As the cells sequentially empty, the cell gas temperatures drops to zero, due to the expansion. TRAC will not allow the gas temperature to drop below zero $\mathrm{C}$. Figure 
4.3-4 shows the pressurizer liquid mass flowrates. Down flow in the pressurizer is negative. As the cells sequentially empty, the mass flowrates jump to zero. Fig. 4.3-5 shows the pressurizer void fractions. The bottom cell remains at zero, and the adjacent cell finishes the transient with a void fraction of 0.5 . The rest of the cells finish at one. Figure 4.3-6 shows surge line pressures. The differences in final pressures are due to elevation differences in the line. Figure 4.3-7 shows the surge line mass flowrates. It takes approximately 1000 seconds or 17 minutes for the pressurizer to drain down. Fig. 4.3-8 shows voids in the surge line. There is a slight amount of voiding early on in the transient. The pipe size was increased from 6 to 10 inches to reduce this voiding. The pressurizer vessel was lowered approximately $4 \mathrm{~m}$. to prevent flashing in the vessel during the expansion process.

\subsection{TRAC Model}

The TRAC model shown in Fig. 4.3-1 is the same as the pressurizer model in the large TRAC system model, with the exceptions that components 201 and 220 are removed and PIPE component 760 is connected to the fixed inlet header, component 380 . A brief description of the number of cells in each component for the entire TRAC model is listed in Table 2.4-2. Further details such as; cell lengths, flow areas, hydraulic diameters, and form loss coefficients can be found in the TRAC model input deck, Appendix A. PIPE components 760 and 762, in Fig. 4.3-1, are potential locations for internal to the cavity breaks, and PIPE component 764 is the potential location for an external break.

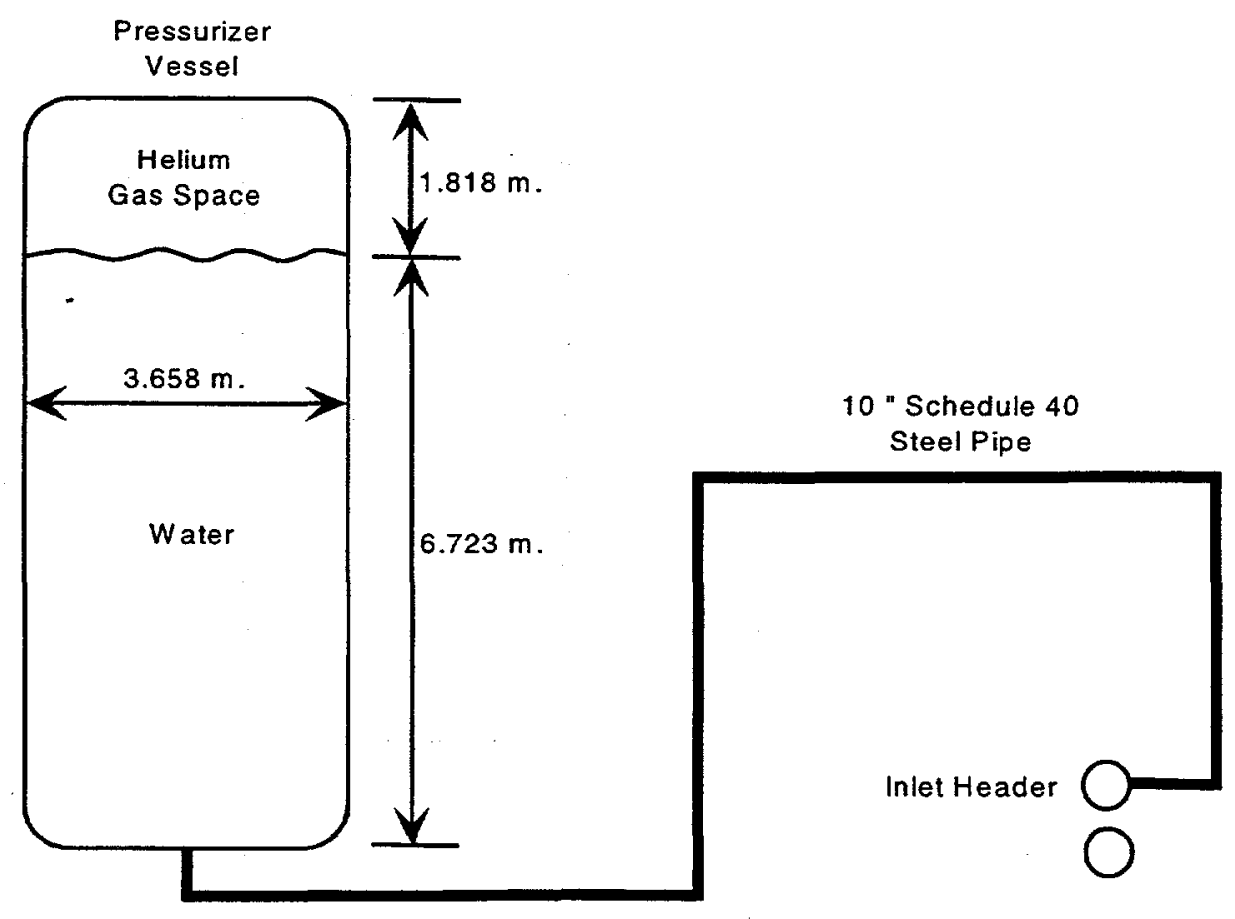

Figure 4.2-1 Schematic of the pressurization vessel and the surge line. 


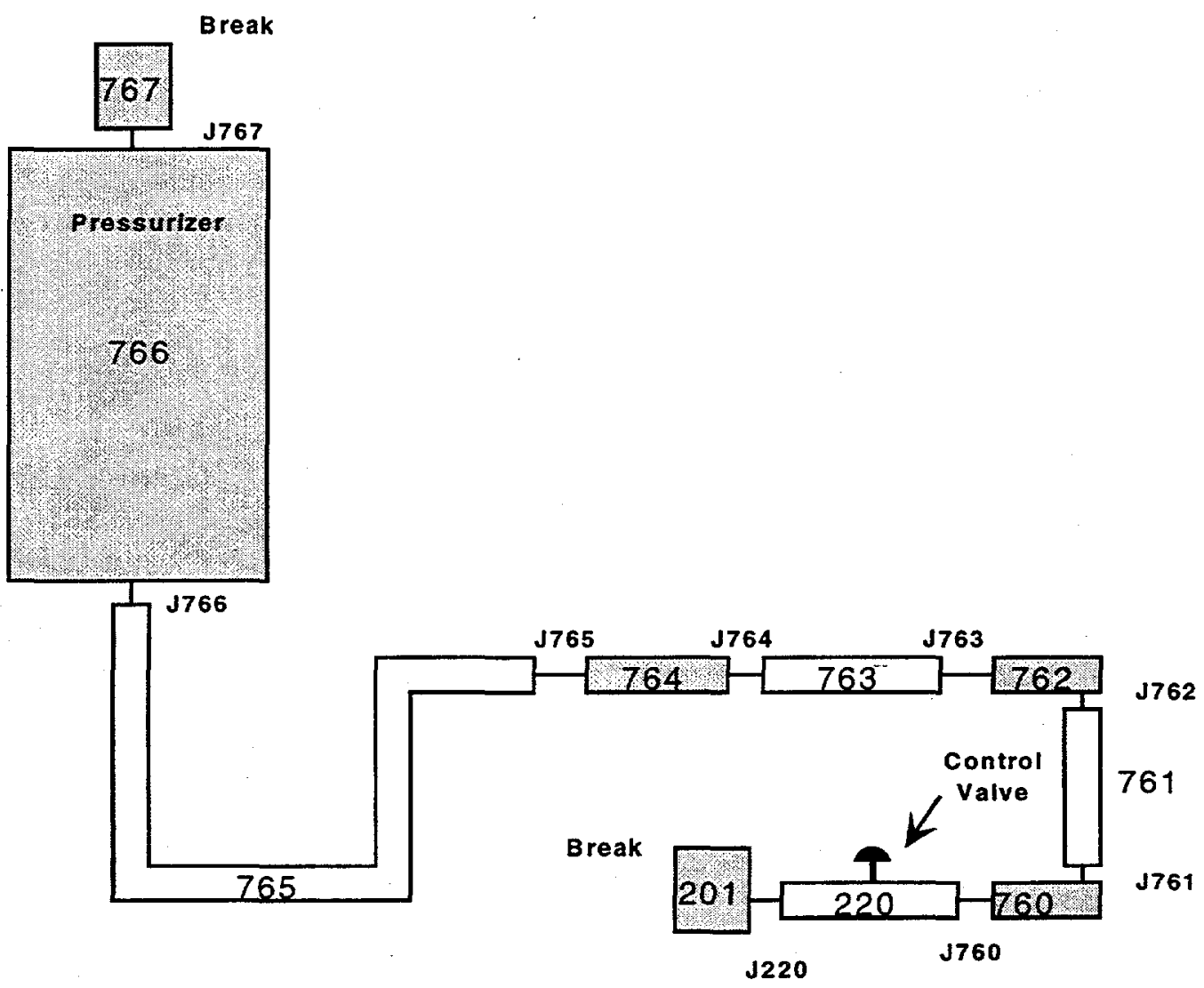

Figure 4.3-1 TRAC component layout of a stand-alone drain down model of the pressurizer and surge line. 


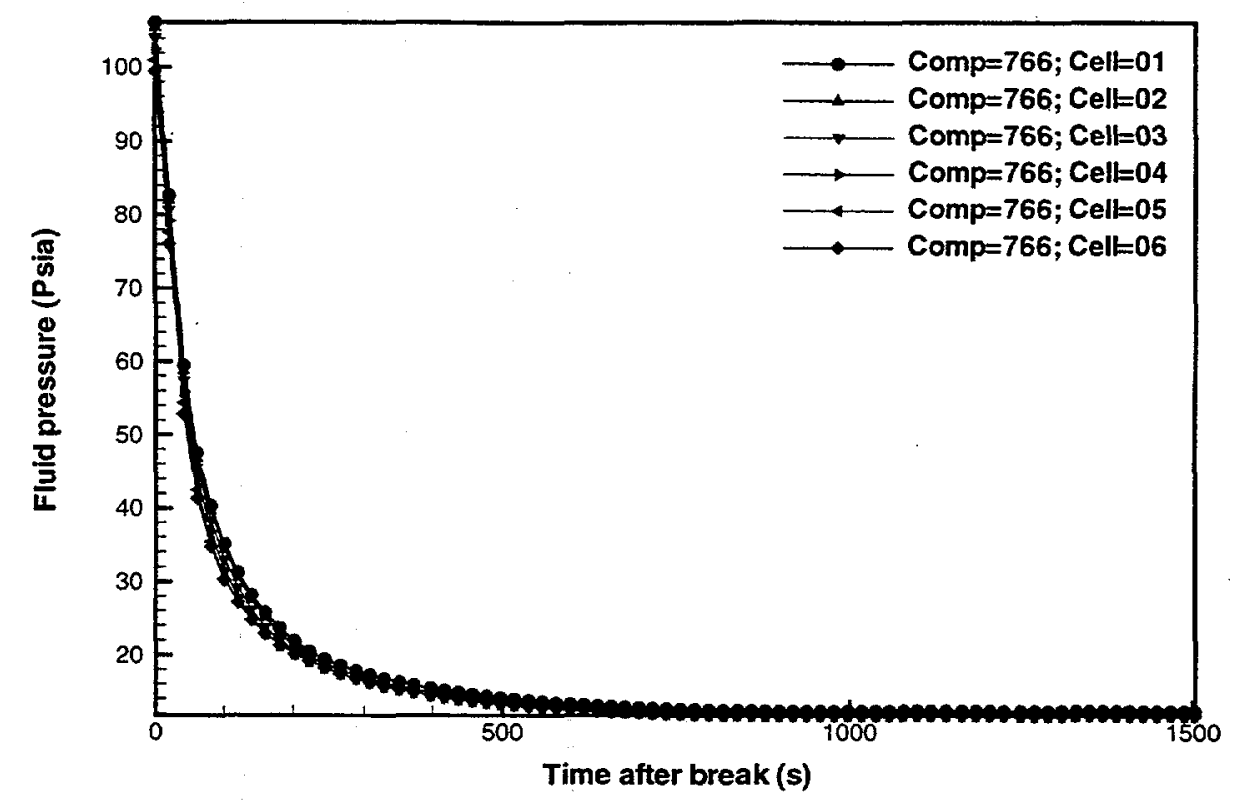

Figure 4.3-2 Pressurizer fluid pressures for a simulated internal large-break LOCA located just above the top shield.

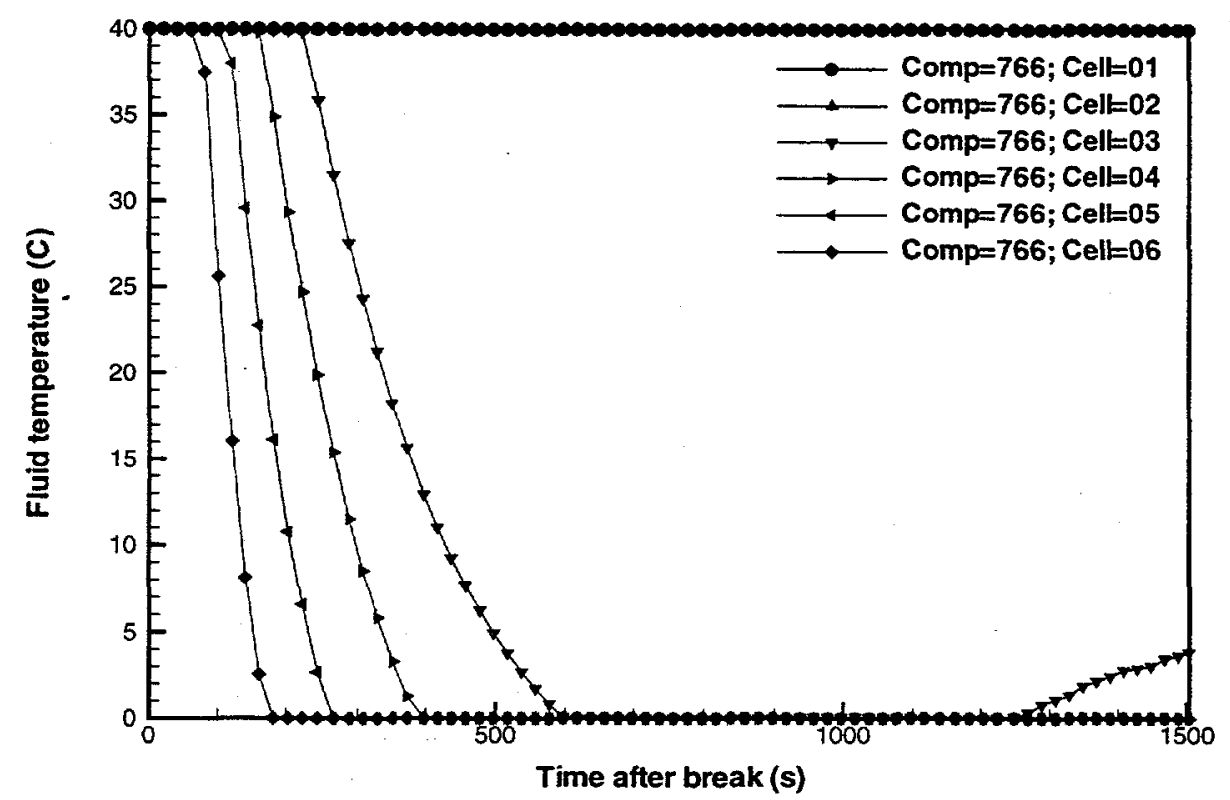

Figure 4.3-3 Pressurizer fluid temperatures for a simulated internal large-break LOCA located just above the top shield. 


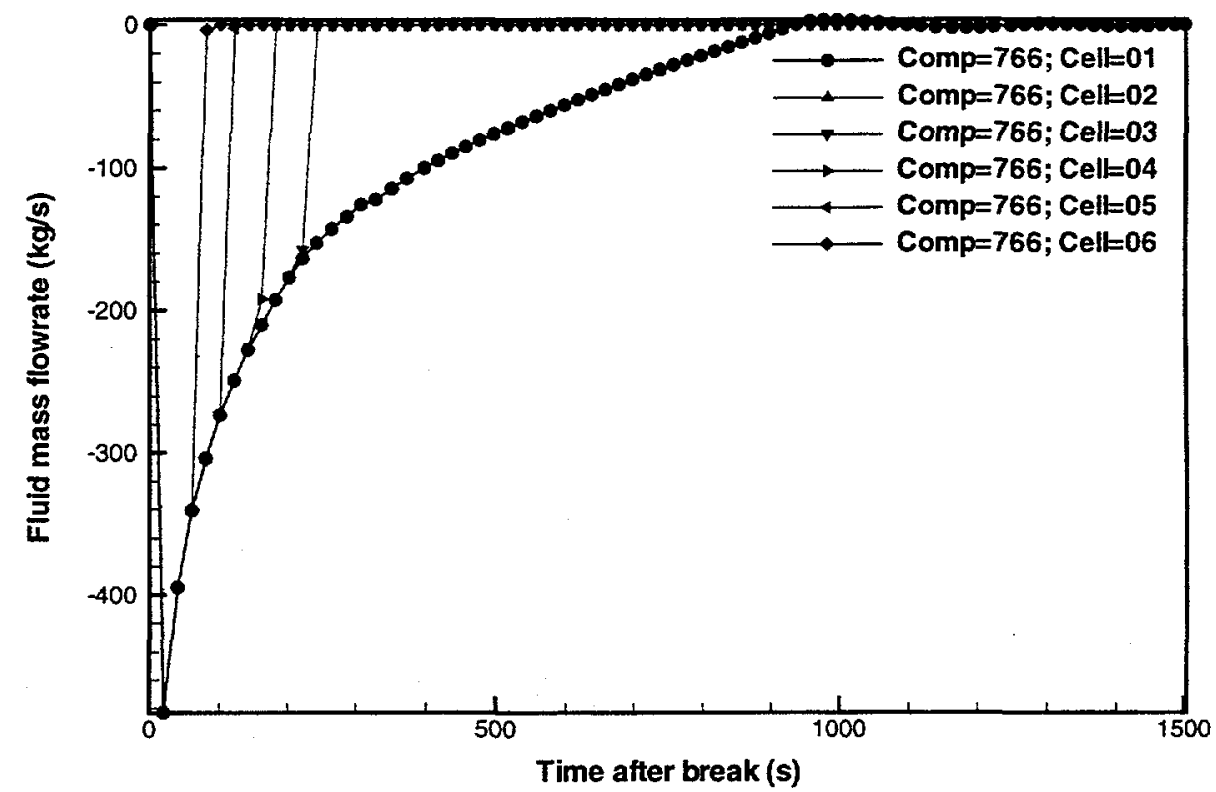

Figure 4.3-4 Pressurizer liquid mass flowrates for a simulated internal large-break LOCA located just above the top shield.

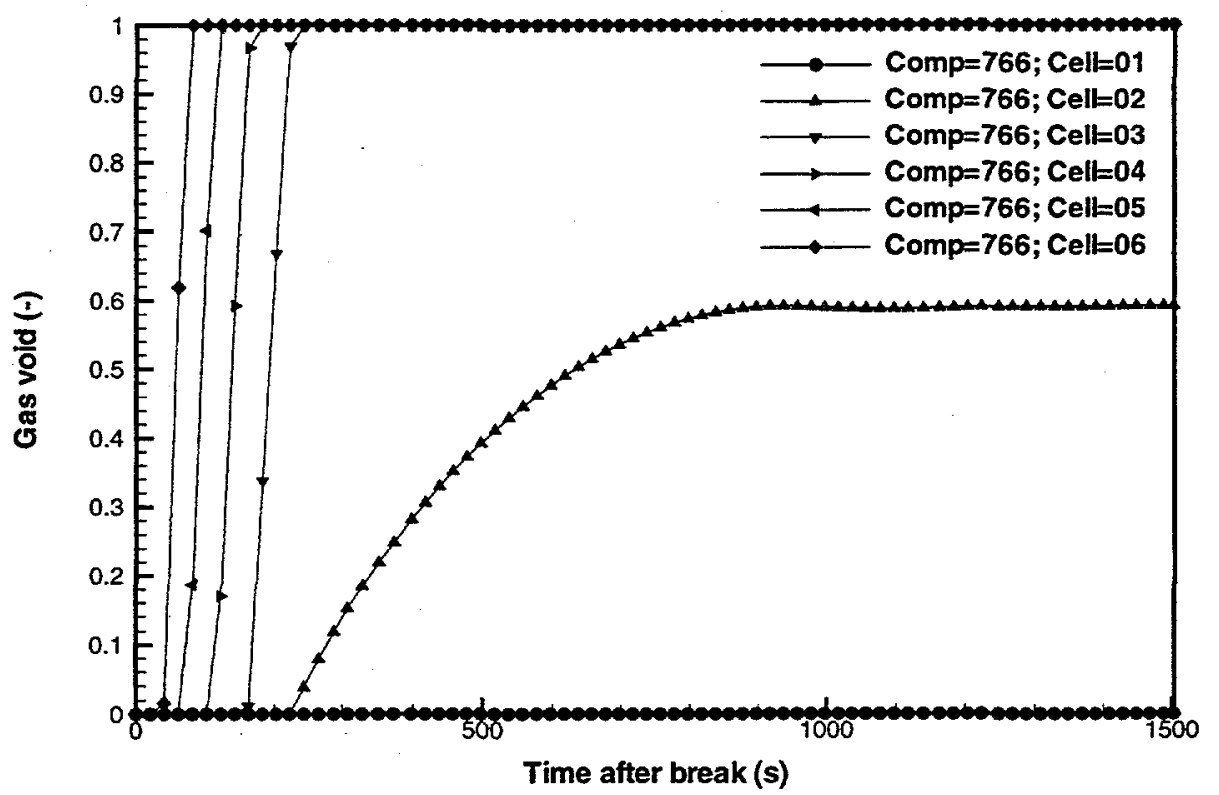

Figure 4.3-5 Pressurizer void fractions for a simulated internal large-break LOCA located just above the top shield. 


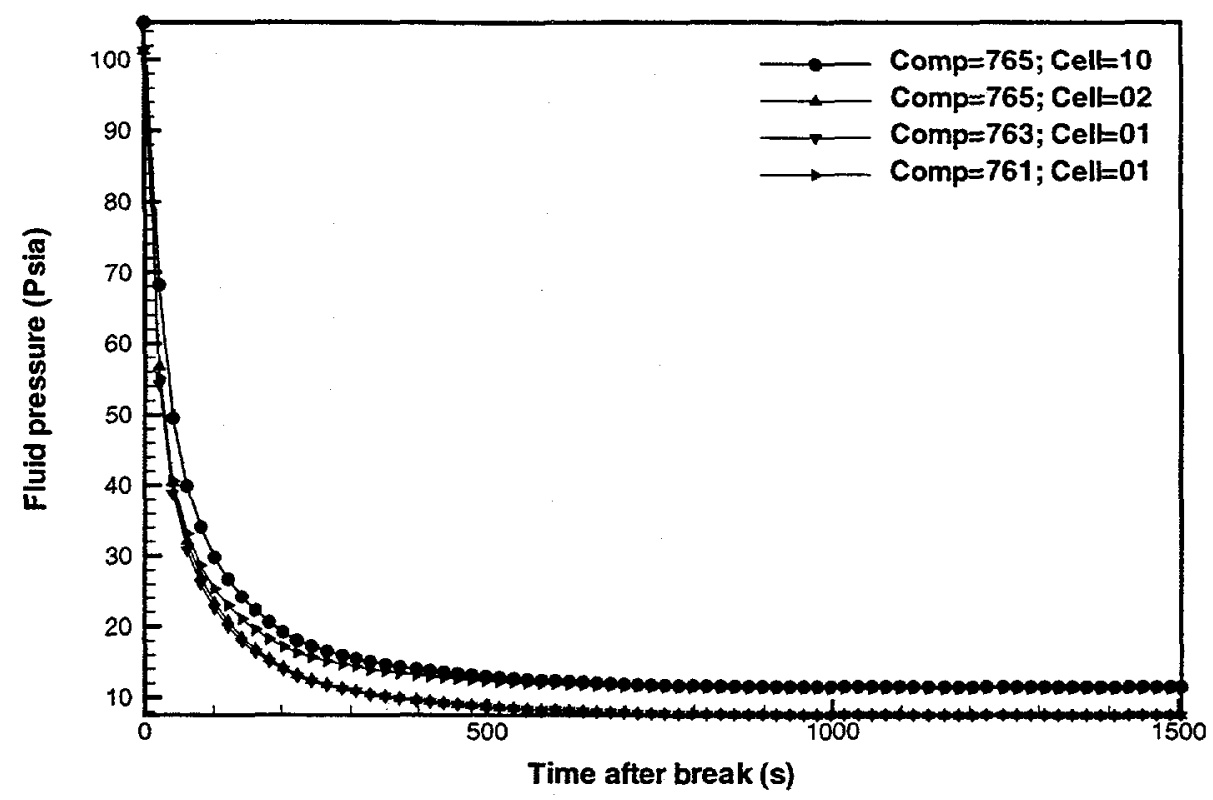

Figure 4.3-6 Pressurizer surge line fluid pressures for a simulated internal large-break LOCA located just above the top shield.

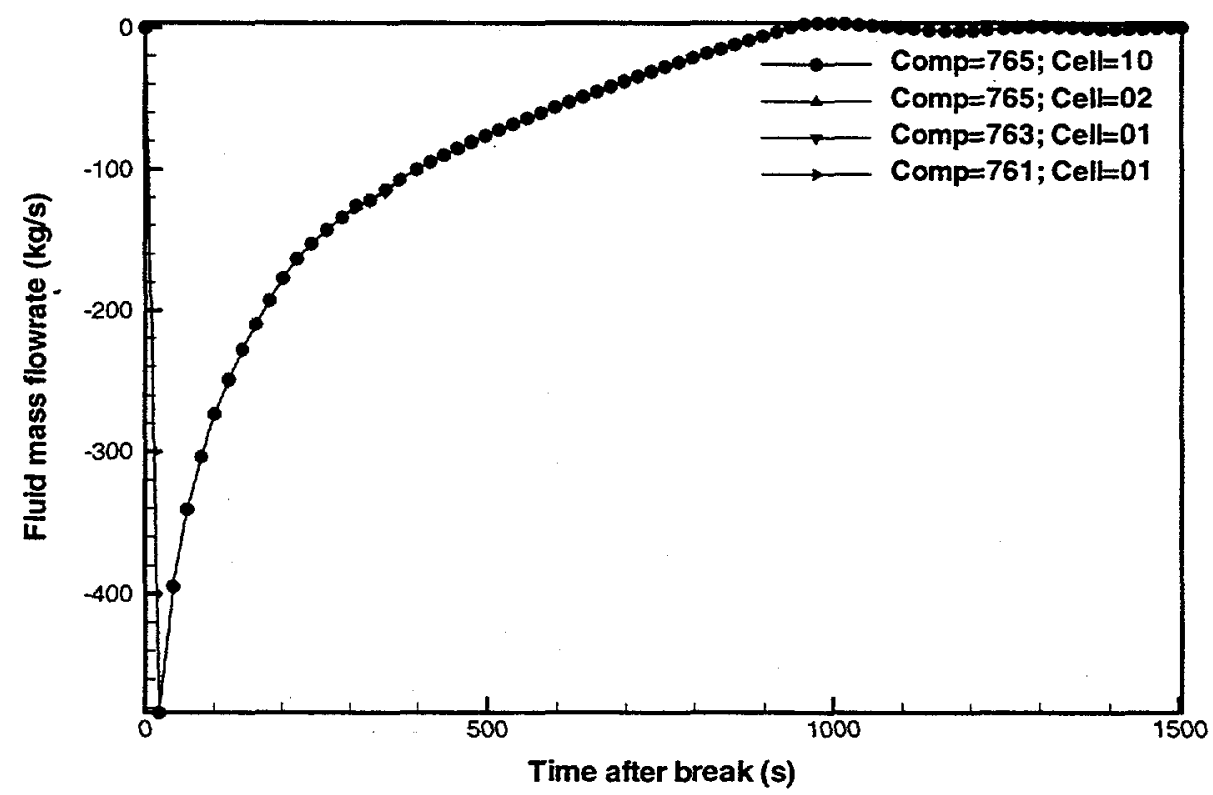

Figure 4.3-7 Pressurizer surge line liquid mass flowrates for a simulated internal largebreak LOCA located just above the top shield. 


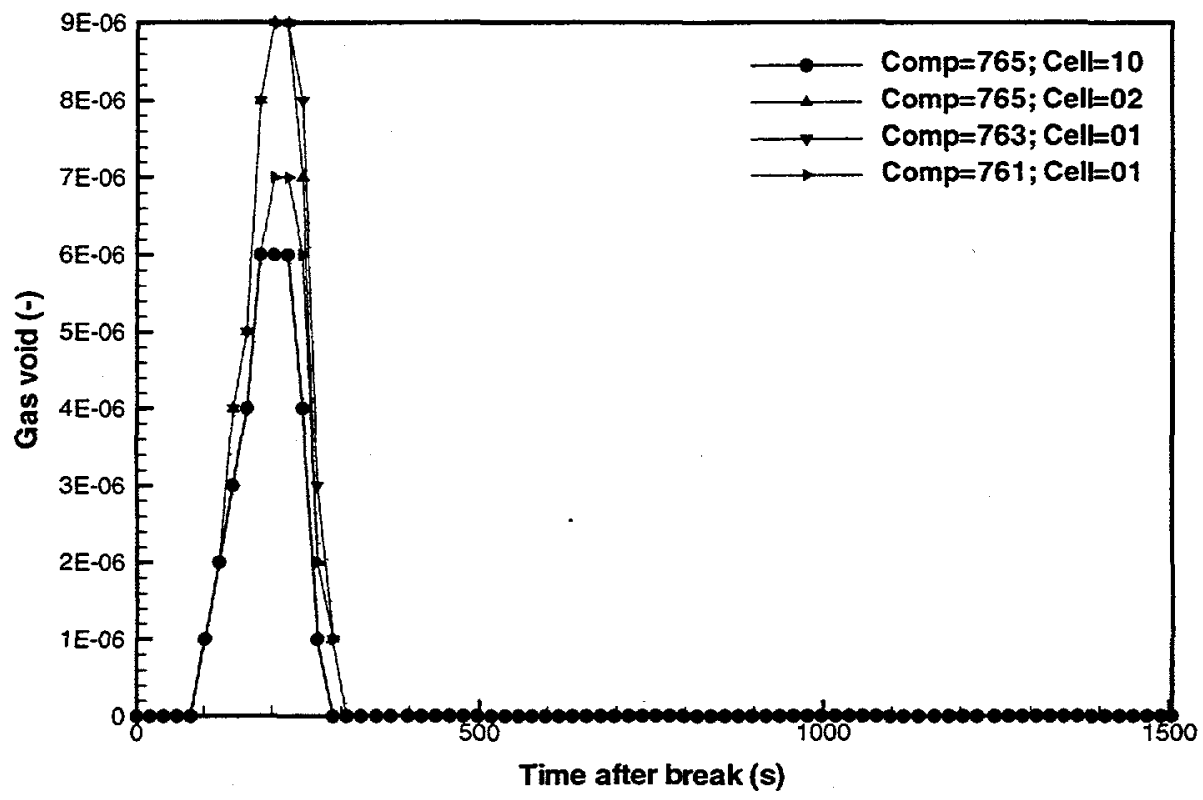

Figure 4.3-8 Pressurizer surge line void fractions for a simulated internal large-break LOCA located just above the top shield. 


\section{$5 \quad$ RHR and Component Descriptions}

\subsection{Introduction}

The purpose of the blanket residual heat removal system is to dissipate energy that is generated within lead blanket components, following an accident that interrupts the primary heat removal system flow. It is designed to remove decay heat. Most of the system piping is external to the cavity vessel, but it connects to the two fixed blanket headers that are inside the vessel. The residual heat removal system piping is parallel to the primary heat removal system, and the design flowrate is between one to four percent of the primary heat removal system flowrate. A check valve ensures that back flow will not occur through the residual heat removal system while the primary system is in operation.

\subsection{Pipe Network Layout}

Figure 5.2-1 is a schematic of the residual heat removal system. It shows the elbows and valves in the pipe network, and also the pump and heat exchanger. The system piping is 6 inch schedule 40 steel pipe. The flow proceeds from the fixed outlet header in the cavity vessel, through the pump and heat exchanger, and back to the fixed inlet header. There are cutout valves on both the suction and discharge sides of the pump and on the discharge side of the heat exchanger. There is also a check valve on the discharge side of the pump.

Figure 1.1-2 is an areal view of the primary and residual heat removal systems, showing the relative placements of the pumps and heat exchangers of the two systems. Also shown are the positions of the cavity vessel and the pressurizer. Figure 5.2-2 shows both areal and side scale views of the residual heat removal system. Lengths of straight pipe are shown in this figure. The residual heat removal system pipes pass through the cavity vessel wall 2 meters above the top fixed header, in the same manner as the primary system pipes.

\subsection{Heat Exchanger Design}

The residual heat removal system has a single pass shell-and-tube heat exchanger. It was designed based on a flowrate of one percent of the primary system flowrate. The blanket decay power drops to less than one percent of full power in the first second following beam trip. The mass flowrate per tube and the axial temperature gradient in a tube are assumed to be the same for both the primary and residual system heat exchangers. The tube diameters and lengths are the same in both systems. There are two passes in the primary system heat exchangers, consequently the effective tube length is twice that of the residual heat removal system heat exchanger. A single pass of both primary heat removal system heat exchangers has 13,816 tubes. Equation 5.3.1 equates the power removed by the residual system heat exchanger to one percent of the power removed by the two primary system heat exchangers. This equation was used to calculate the number of tubes in the residual heat removal system heat exchanger (277 tubes).

$$
N t_{R H R}\left(\frac{\dot{m}}{t u b e}\right) C_{p} \frac{d T}{d z} L_{R H R}=0.01\left(N t_{P H R}\right)\left(\frac{\dot{m}}{t u b e}\right) C_{p} \frac{d T}{d z} L_{P H R}
$$



where:

$$
\begin{aligned}
& L_{P H R}=2\left(L_{R H R}\right) \\
& N t_{P H R}=13816
\end{aligned}
$$

Page:

The ratio of the total tube cross-sectional area to the tube sheet area is assumed to be the same as in the primary system heat exchangers. The shell diameter is consequently $0.406 \mathrm{~m}$. Figure $5.3-1$ is a schematic of the heat exchanger.

\subsection{Pump Head Curve}

The pump head curve is based on the LOFT homologous head curve. The reference state parameters with which the head curve is normalized are:

$\begin{array}{ll}\text { pump speed } & 1000 \mathrm{rpm} \\ \text { volumetric flowrate } & 0.079838 \mathrm{~m} 3 / \mathrm{s} \\ \text { total dynamic head } & 14.8392 \mathrm{~m}\end{array}$

These values were chosen such that, at a residual system flowrate of $5 \%$ of the primary system full flowrate, the pump speed is $1000 \mathrm{rpm}$. The first quadrant of the LOFT normalized homologous head curve is shown in Fig. 3.4-1. This is the normalized homologous head curve for the primary system pumps. While the primary and residual heat removal systems ust the same normalized head curves, the reference states are significantly different for the two pump types.

\subsection{Steady-State Hydraulic Model}

A simple steady-state hydraulic model, similar to that developed for the primary heat removal system, was developed for the residual heat removal system. This model was developed to determine the necessary pipe size, and to compare with TRAC model results. The model RHRTRC applies the mechanical energy equation to five sections of the system sequentially: from the heat exchanger discharge to the fixed inlet header, across the heat exchanger, between the pump and heat exchanger, across the pump, and from the fixed outlet header to the pump. The independent variables in this model are: the volumetric flowrate, the pump speed, and the inlet header pressure. The model calculates at the outlet header and desired intermediate points in the system.

Since the portion of the heat removal system inside of the cavity vessel that contains the blanket modules is not modeled, the system demand curve is indeterminate. The dynamic pressure drop between the fixed headers is assumed to be proportional to the square of the flowrate, equation 5.5.1, and at full primary heat removal system flow of $1569 \mathrm{~kg} / \mathrm{s}$, the pressure drop from the inlet to outlet header is approximately 41 psid. This operating point was used to calculate the proportionality constant. The pressure drop between the headers, as a function of mass flowrate, is evaluated with equation 5.5.2.

$$
\begin{gathered}
\Delta P_{d y n .}=K(\dot{m})^{2} \\
P_{I H}-P_{O H}=K(\dot{m})^{2}-\rho g \Delta z
\end{gathered}
$$

At two percent flow, the inlet minus outlet header pressure drop is $-1.052 \mathrm{psid}$, and at four percent flow the pressure drop is -1.002 psid.

The fixed inlet header pressure, that would occur in the event of a large break LOCA, is a function of time and the break location. The transient is due in part to the pressurizer 
TRAC BLANKET SYSTEM MODEL

Page:

flow. For breaks external to the cavity vessel, the minimum pressure will occur after the pressurizer flow ceases, and there is no longer flow out of the inlet header through the primary system pipe. Figure 5.5-1 is a schematic of a break in the primary system piping at the point where the heat exchanger discharge piping passes through the cavity vessel wall. The pressure at the break location is atmospheric, and there is a stagnant column of water in the vertical primary system pipe that connects to the inlet header. The hydrostatic head establishes the inlet header pressure at $19.3 \mathrm{psia}$. This is the inlet header pressure boundary condition in the steady-state model. A break internal to the cavity would eventually be submerged by the cavity flood system, and water would flow into the heat removal system through the break, a different situation altogether.

A code listing of RHRTRC and also input and output files are included in Appendix D. A script that is used to run RHRTRC on a SGI work station is also included.

\subsection{Pipe size Design Study}

RHRTRC was used to size the pipe in the residual heat removal system, with the criteria that the pump not cavitate at flowrates below $4 \%$ of the primary system flowrate. Equation 5.6.1 is the relation for the required NPSH [1]. This relation is based on data from over 600 small centrifugal pumps from different manufacturers. The Bingham pump NPSH relation was not used because of scaling problems due to the great difference in capacities.

$$
N P S H_{R}=N P S H_{\text {ref }}\left(\frac{\Omega}{\Omega_{\text {ref }}}\right)\left(\frac{Q}{Q_{\text {ref }}}\right)^{.424}
$$

The reference parameters are:

$\begin{array}{ll}\text { NPSH } & 2.35 \mathrm{~m} \\ \text { pump speed } & 1760 \mathrm{rpm} \\ \text { flowrate } & 0.02778 \mathrm{m3} / \mathrm{s}\end{array}$

Schedule 40 steel pipes with diameters from 3 to 6 inches were considered. Table 5.6-1 shows the available and required values of NPSH as functions of the the pipe diameter and the percent full flow. The 6 inch pipe is the only one to satisfy the criterion that cavitation not occur at $4 \%$ of full flow. The threshold for cavitation in 6 inch pipe occurs at $5.4 \%$ of full flow.

\subsection{TRAC Model steady-State Benchmarking}

Table 5.7-1 shows a comparison between pressures in the residual heat removal system predicted by the steady-state model RHRTRC and a stand-alone TRAC model of the system. The flowrate is $62.912 \mathrm{~kg} / \mathrm{s}$ ( $4 \%$ of full flow) and the pump speed is 897 rpm. The agreement between the two code results is excellent. This stand-alone TRAC model was incorporated into the blanket heat removal system model.

\subsection{TRAC Model Component Layout}

Figure 5.8-1 shows the TRAC component layout and junction numbers for the blanket residual heat removal system. A brief description of and the number of cells in each component of the entire TRAC model is listed in Table 2.4-2. Further details such as 
TRAC BLANKET SYSTEM MODEL

cell lengths, flow areas, hydraulic diameters, and form loss coefficients are found in the TRAC model input deck (see Appendix A).

Figure 5.8-1 does not show the heat exchanger secondary flow and heat structure components. Component 630 is the pump, component 640 contains the check valve, that opens to enable the residual heat removal system, and component 652 models the heat exchanger tubes. PIPE components 624 and 661 are potential break locations that are external to the cavity vessel. PLENUM components 380 and 340 are respectively the inlet and outlet fixed headers.

\subsection{References}

1. S. Yedidiah, Centrifugal Pump Problems, Causes and Cures, Petroleum Publishing Co., 1980.

Table 5.6-1 Estimated required and available NPSH for RHR piping.

\begin{tabular}{|c|c|c|c|}
\hline Pipe Diameter & \% Full Flow & NPSH Available $(\mathbf{m})$ & NPSH Required $(\mathbf{m})$ \\
\hline 3 inch & 2 & -20.71 & 1.46 \\
\hline 4 inch & 4 & -22.04 & 2.50 \\
\hline 5 inch & 4 & -0.067 & 1.95 \\
\hline 6 inch & 5 & 3.87 & 2.09 \\
\hline
\end{tabular}

Table 5.7-1 Comparison between TRAC and simple hydraulic model pressure profile for RHR piping loop.

\begin{tabular}{|c|c|c|}
\hline Location & RHRTRC pressure (psia) & TRAC pressure (psia) \\
\hline Outlet Header & 20.299 & 20.335 \\
\hline Pump Suction & 11.843 & 11.775 \\
\hline Pump Discharge & 28.799 & 28.705 \\
\hline Heat Exchanger Inlet & 28.038 & 27.990 \\
\hline Heat Exchanger Discharge & 21.880 & 22.060 \\
\hline Inlet Header & 19.295 & 19.295 \\
\hline
\end{tabular}




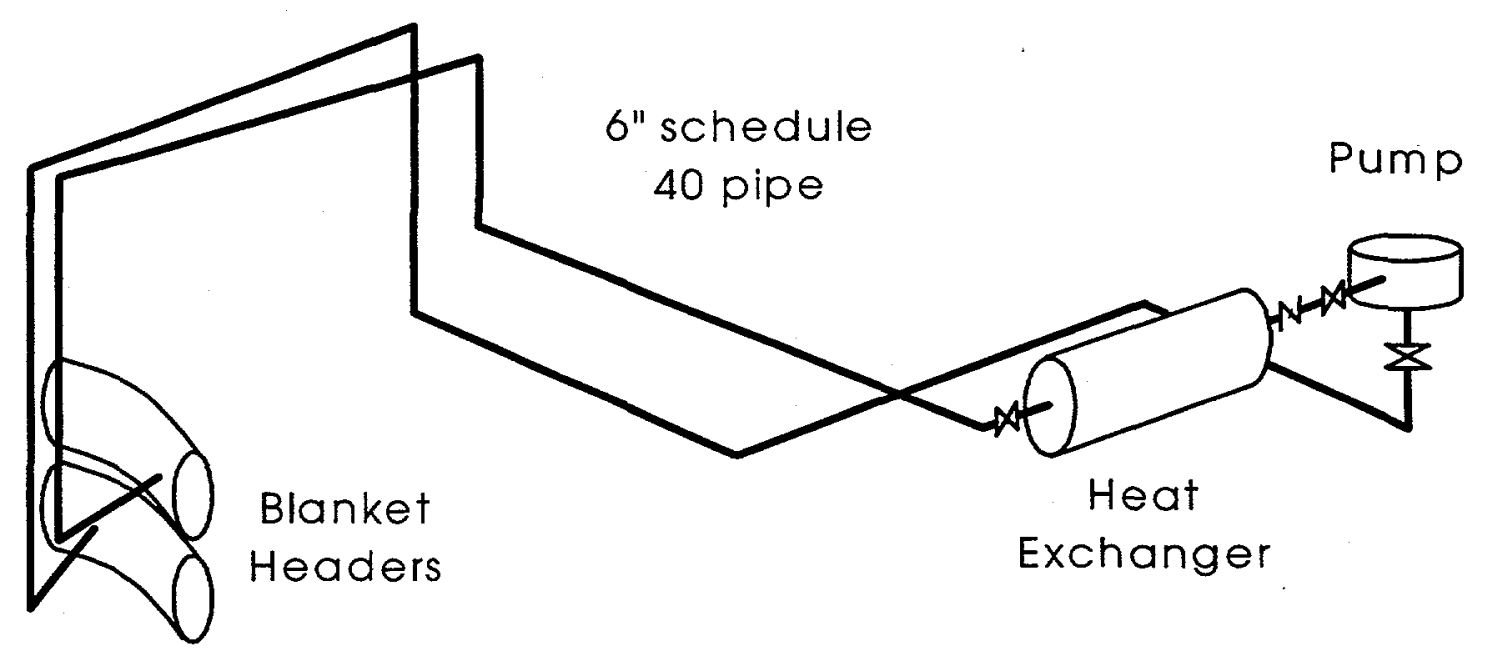

Figure 5.2-1 Schematic of the blanket residual heat removal system. 


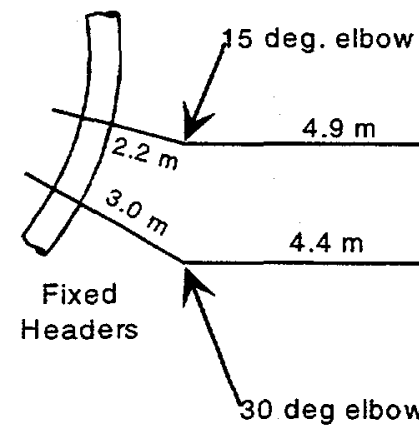

$14.2 \mathrm{~m}$

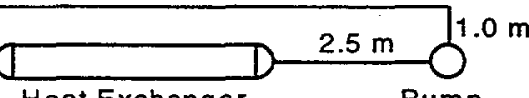

Heat Exchanger Pump

Heat Exchanger

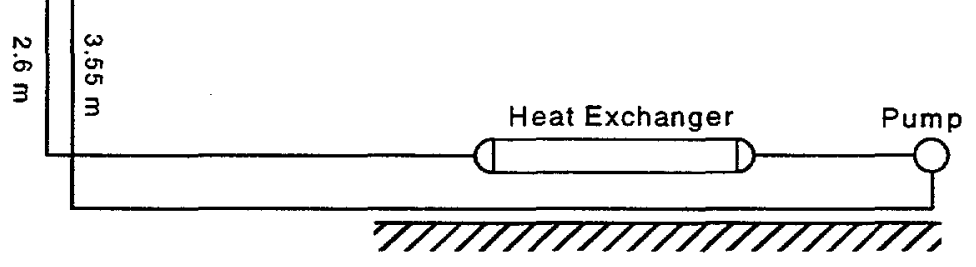

Side View

Figure 5.2-2 Areal and side views of the residual heat removal system. 


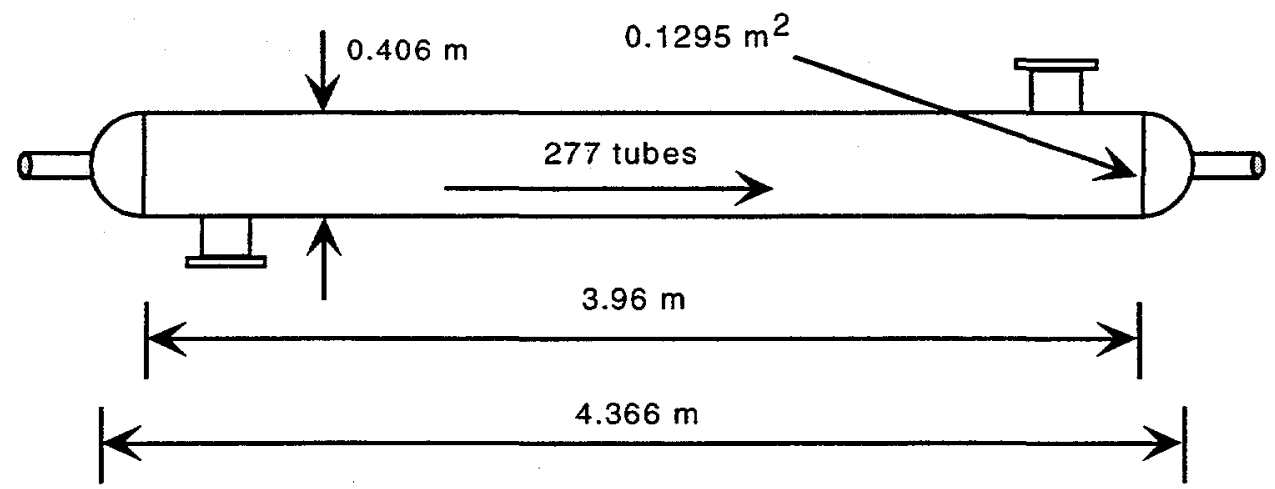

Figure 5.3-1 Schematic of the blanket residual heat removal system heat exchanger. system.
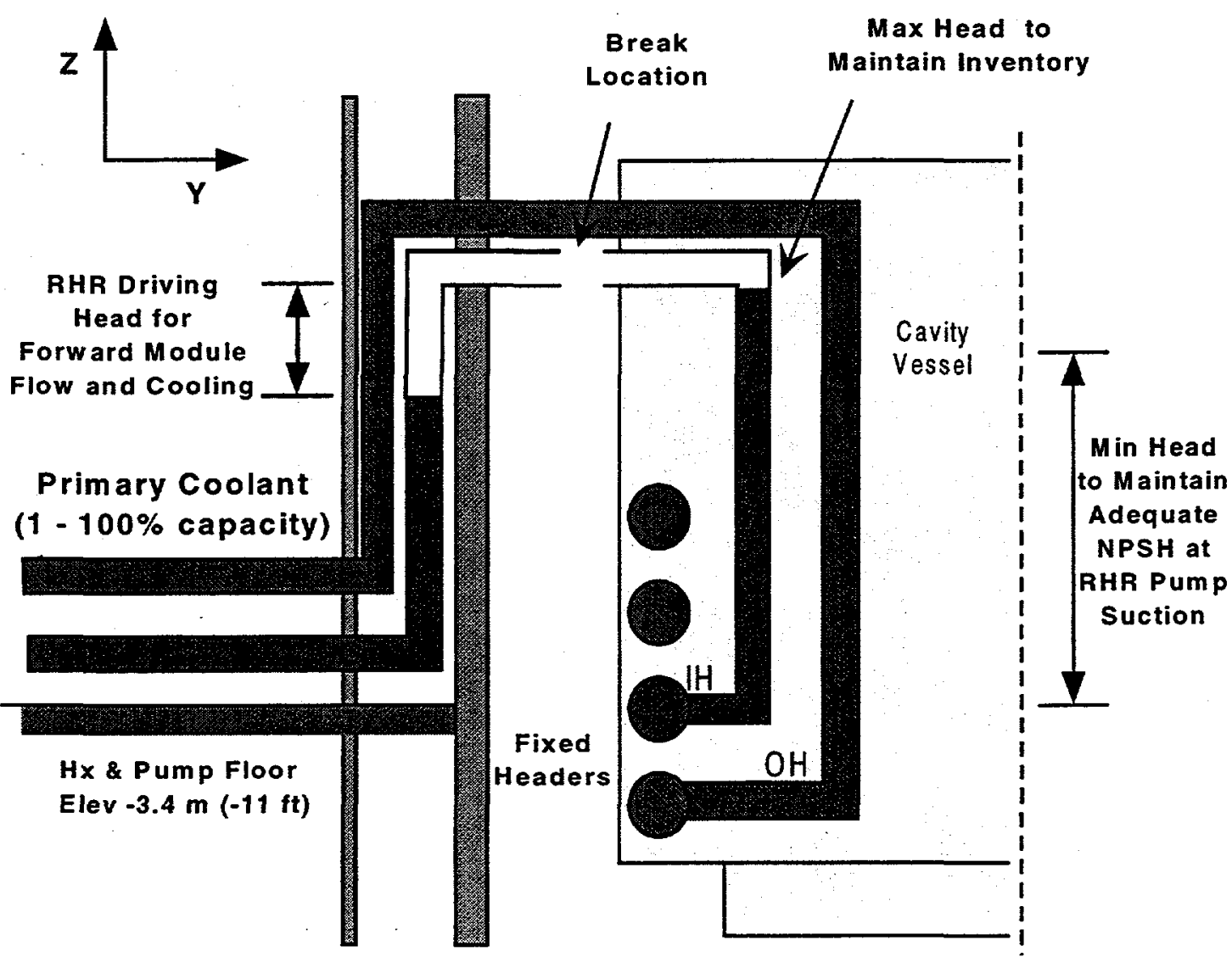

Figure 5.5-1 Schematic of the break location in the primary heat removal system that was used to determine the fixed inlet header pressure boundary condition for the residual heat removal system simple hydraulic model, (RHRTRC). 


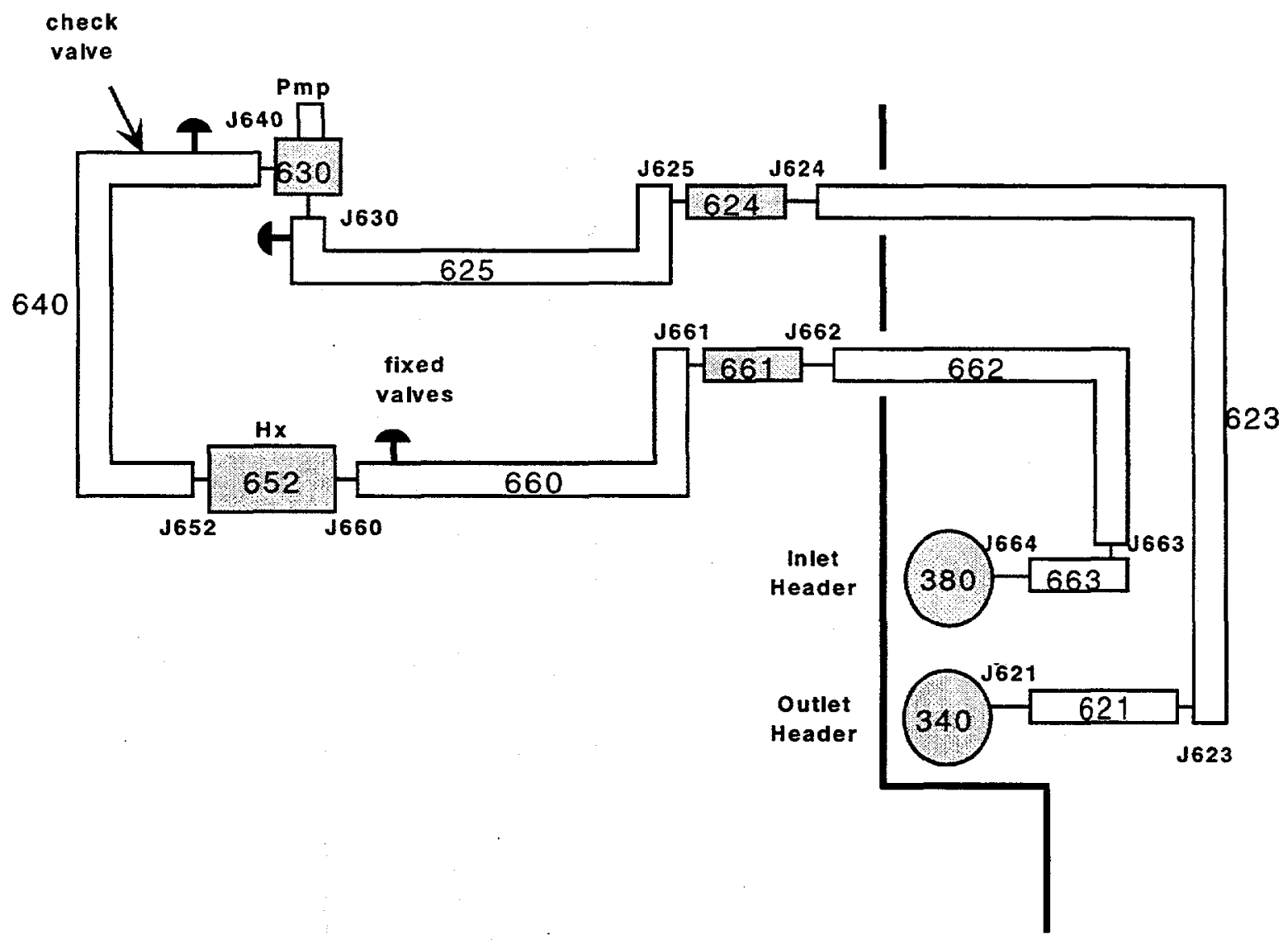

Figure 5:8-1 TRAC model component layout of the blanket residual heat removal system. 


\section{Cavity Vessel/Flood System Descriptions}

Since a variety of design improvements for the primary coolant systems are currently being considered by General Atomics (GA), LANL, and SRTC personnel, internal break loss-of-coolant accidents will be re-addressed at a later date when proposed design modifications can be tested with the integrated system model. The following discussions apply to the current cavity vessel and cavity flood models incorporated into the system TRAC model.

\subsection{Background}

A TRAC cavity flood system model consisting of the cavity vessel, VALVE components to simulate internal breaks, and a cavity flood pool was developed. The target system's original cavity flood system model was not directly applicable to the blanket system. Potential break locations differ between the target and blanket systems. Also, thermal connections of the blanket heat structures require a different spatial nodalization of the cavity vessel. To accommodate various potential break locations, VALVE components were employed to connect the primary heat removal and individual blanket module coolant lines to the cavity vessel.

The current cavity flood system model for blanket system safety analyses does not explicitly account for potential heat sources or sinks with regard to the neighboring target ladders that are also housed within this cavity vessel. Under those accident scenarios where the cavity vessel is not flooded, thermal radiation and gas-phase natural convection are the predominate means for energy exchange. In most cases these modes for energy transfer will be negligible. Under flooded cavity scenarios it is assumed that the net energy generated in and removed from the target ladders is reasonably balanced and imposes no net impact on the cavity flood water.

During the initial phase of development of the primary HR piping models, it was determined that TRAC's TEE component results in a significant over-prediction of hydraulic losses. Knowing this, all TEE components existing in the original target cavity system model were replaced using an appropriate combination of PLENUM and PIPE components.

\subsection{Cavity Vessel}

The overall geometric features, such as flow areas and volumes of the cavity vessel, are essentially identical with the values used in the target cavity pool model.

\subsubsection{Thermal Coupling of Heat Structures}

As mentioned above, modifications to the target cavity vessel model were required to accommodate the axial locations of the 6 lumped blanket modules. The TRACPF1/MOD2 code version 5.4.28a [1] was used to model the blanket system. This specific version of TRAC has a limitation on how multiple heat structures can be connected to a common fluid component (i.e., in this case pipe components). This limitation applies to PIPE components that are vertically oriented. As shown in Fig. 6.21 the lower portion of the cavity vessel is modeled with a vertically oriented PIPE component labeled 823. Blanket modules 1 through 5 were divided into axial cells 
consistent with the axial cells chosen for component 823. Due to the low power and flow demands of module 6 (note that module 6 is a composite of various spatially complex modules), module 6 remains thermally isolated from the cavity vessel gas space.

The darker shaded HEAT STRUCTURES shown in Fig. 6.2-1 represent the lead components cladded with aluminum (i.e., components 951, 966, 955, 961, 978, 962, $979,963)$. The lighter shaded HEAT STRUCTURES represent the aluminum structures located between the various coolant flow channels and the cavity vessel gas space (i.e., components $984,901,916,905,988,911,931,912,932$, and 913). Solid lines have been drawn between these aluminum structures and the lower cavity gas space to indicate the thermal connections made in the cavity vessel model. Depending upon the aluminum structure considered, 5 to 6 axial connection per heat structure exists.

\subsubsection{Cavity Vessel Vent Valve}

In the upper region of the cavity vessel dome a control valve (i.e., component 828 ) exists. A control valve was placed here to allow pressurization of the cavity vessel gas space once a LOCA has been determine. Above the control (vent) valve a vent line saturated with air exists that emanates from a common vent line system ultimately connected to the building HEPA filter systems. The control valve and vent line were assumed to have the size of a 12 inch schedule 40 pipe. The control valve is actuated regardless of the location of the LOCA line break (i.e., internal or external). The current trip signal logic is based on a $5 \%$ reduction in system pressure in the pressurizer surge line next to the fixed inlet header. A one second valve opening time is assumed.

\subsubsection{Potential Break Locations within Cavity Vessel}

In Fig. 6.2-2 a more detailed view of the cavity vessel model can be seen. Three basic vertical sections exist within this vessel model (i.e., a lower, middle, and upper section). As discussed above, the lower section contains the thermal connections made between the cavity vessel gas space and the various blanket modules (1 through 5 ). Numerous narrow passageways (i.e., approximately one inch gaps) exist within this section of the cavity vessel. The fill line is connected to the bottom of this lower section as illustrated in Fig. 6.2-2.

The middle section represents the area just above the top shields where a significant increase in cross-sectional flow area occurs. In this section potential break locations have been placed to accommodate large break LOCA's associated with individual modules. Due to the confined geometric arrangements below the top of the shields line, breaks situated in the lower section would result in much smaller flowrates and shorter times to cover. Potential break locations have been placed in each of the 6 modules just above the top of the shields. As shown in Fig. 6.2-2, VALVE components 840 and 842 connect to each side of a module break and also then connect to the cavity space in PLENUM component 824 (note that the 201 through 204 BREAK components shown are used only when isolated test runs of the cavity flood system are being performed).

In the upper section of the cavity vessel similar CONTROL VALVE components are used to simulate a potential break in the primary $\mathrm{HR}, \mathrm{RHR}$, or pressurizer lines entering into the fixed inlet header (see components 850 and 852 in Fig. 6.2-2). As shown in Fig. 6.2-2, VALVE components 850 and 852 connect to each side of a primary loop pipe 
break and also then connect to the cavity space in PLENUM component 826. The very top of the upper section within the cavity dome connects to the controlled vent valve (component 828).

\subsection{Cavity Flood Pool}

The geometric features of the cavity pool are essentially identical to the values used in the target cavity pool model. The TEE component was replaced with a PLENUM component as stated above. The cavity flood pool is presented in Fig. 6.2-2. The upper portion of the cavity flood pool is filled with saturated air emanating from a common vent line ultimately connected to the building HEPA filter systems.

\subsection{Cavity Flood Valve and Fill Line}

Modifications were made to the flood fill line by moving the flood control valve down to the lower portion of the fill line. Line lengths and fluid volumes were preserved. By lowering this flood valve, pool water reaches the cavity vessel in a shorter time and also eliminates the difficult task of flooding the vertical section of a fill line initially filled with a gas. The cavity flood valve and fill line are also presented in Fig. 6.2-2.

Upon receiving a signal to open the control valve, the valve fully opens within a 1 second time period. The actuation signal chosen is based on a 1.5 meter high liquid inventory at the base of the cavity vessel gas space. This particular signal was chosen to ensure that no inadvertent flood valve openings would occur. Once actuated, the flood valve fully opens and remains open throughout the remainder of the transient. For the massive blanket modules housed within the cavity vessel, thermal inertia is sufficiently large such that rapid flooding of the cavity space is unnecessary.

\subsection{Simple Vapor-Liquid Equilibrium (VLE) Model}

A simple standalone model based on the control volume approach was also developed to provide supporting analyses and insight into the cavity flooding process. The TRAC code has demonstrated trouble modeling pipe break flows into cavities when the cavity pressure is below and the nominal APT cavity pressure is on the order of 0.1 psia. The control volume model (based on thermodynamic vapor-liquid equilibrium) was used to help understand the implications of assuming an initial cavity pressure of 5 psia, currently used in the TRAC system model.

Under normal operating conditions the cavity vessel will be operating between 50 to 60 $\mathrm{C}$, while the cavity flood pool will be seating at or below the maximum expected building temperature of approximately $40 \mathrm{C}$. The saturation temperature of water at a vapor pressure of 5 psia is approximately $32.7 \mathrm{C}$.

The simple VLE model predicts cavity vessel saturation to occur very rapidly following an internal break (i.e., on the order of 0.2 seconds). Initially, the cavity vessel contains a low pressure unsaturated gas mixture. The rapid evaporation process results in an early on cavity vessel gas temperature drop to approximately $27 \mathrm{C}$ with a corresponding saturated gas pressure of 3.4 psia. Without opening the vent valve at the cavity dome, the cavity gas pressure would only slowly rise as the gas space within the cavity vessel is compressed during the fill process. However, opening of the vent valve results in a fairly rapid process of pressurizing (i.e., on the order of minutes). The impact of the initially slightly higher cavity pressure in the TRAC model (5 versus 3.4 psia) is minimal 
since near choke flow conditions exist at the break location and the initial driving force for cavity flood is significantly larger.

\subsection{References}

1. Safety Code Development Group, "TRAC-PF1/MOD2: An Advanced Best Estimate Computer Program for Pressurized Water Reactor Thermal-Hydraulic Analysis," Los Alamos National Laboratory report LA-12031-M, Vol. 1 (NUREG/CR-5673), (July 21, 1993). 


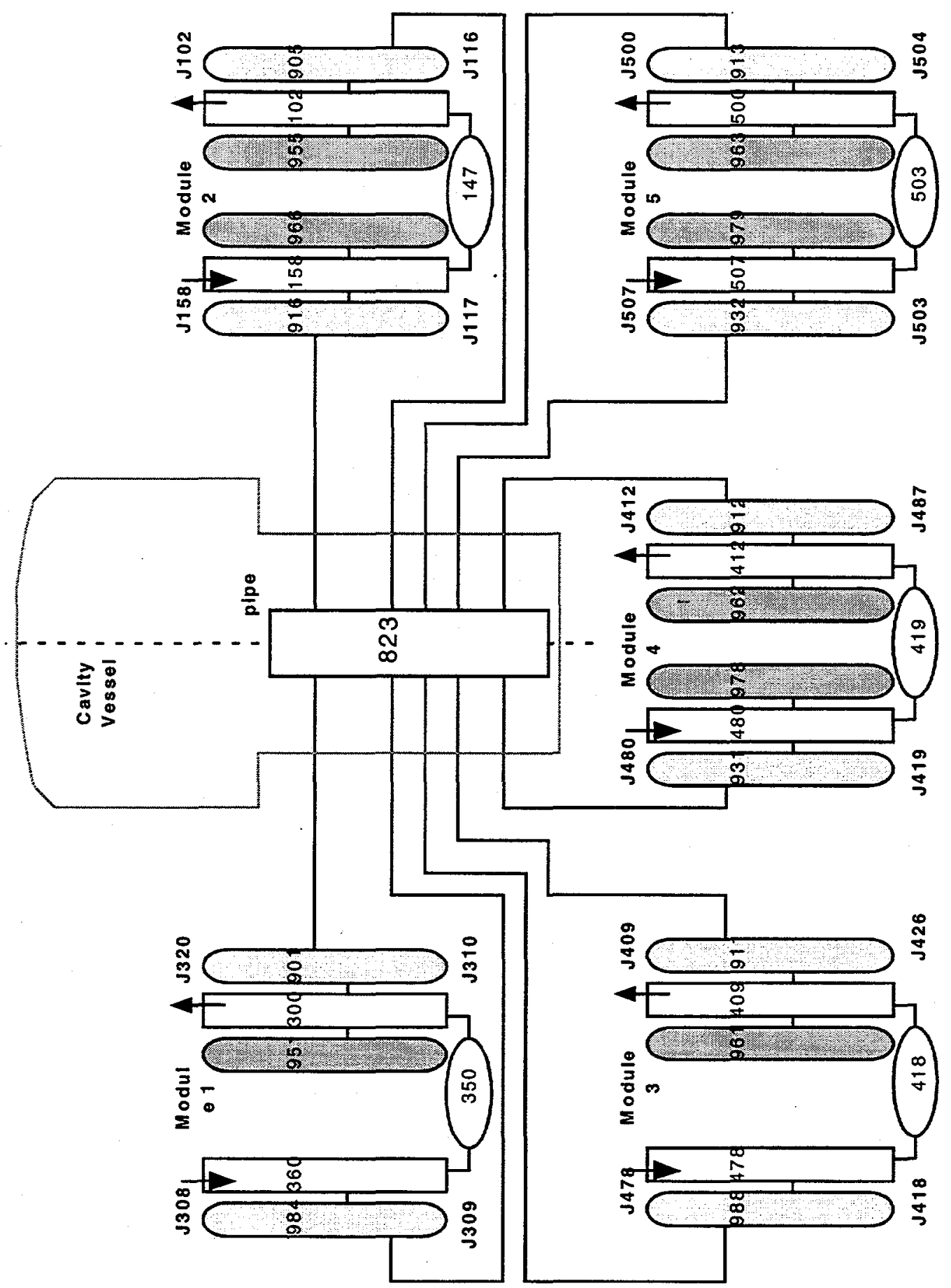

Figure 6.2-1 TRAC component layout for the cavity vessel and blanket module heat structures. 


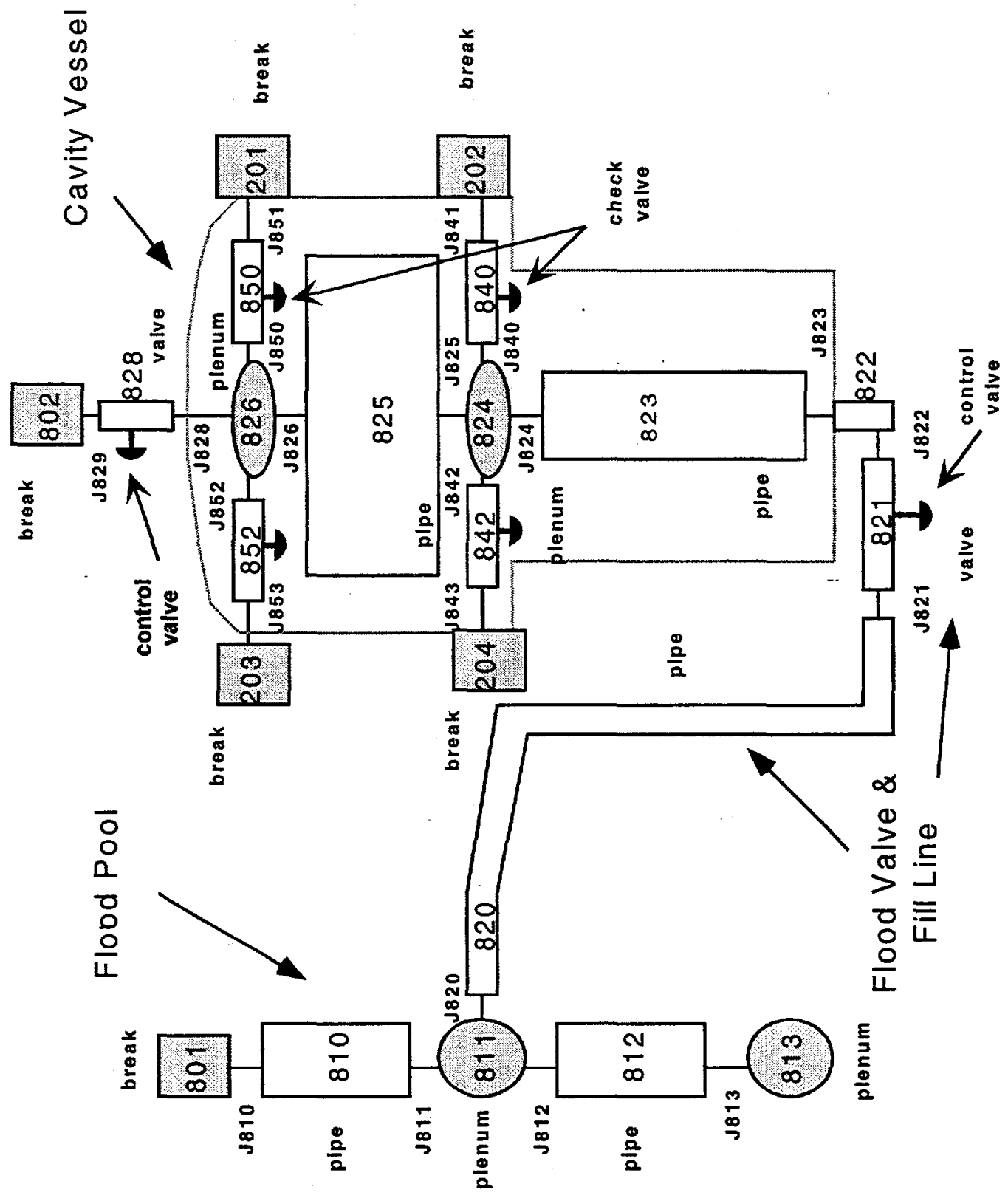

Figure 6.2-2 TRAC component layout for the cavity vessel and cavity flood system. 


\section{Secondary HR and RHR Component Descriptions}

\subsection{Introduction}

Major components of a typical blanket heat removal system include heat exchangers, piping, and pumps. The blanket heat removal system is comprised of the primary heat removal (HR) system, the secondary heat removal system, the primary residual heat removal (RHR) system, and the secondary RHR system. These systems provide the heat removal function for all the thermal energy deposited within the blanket modules. The blanket coolant system has one primary HR, one secondary HR, two $100 \%$ primary $\mathrm{RHR}$, and two secondary RHR systems. The blanket module system is connected to a single main primary loop, with three $50 \%$ of total capacity pumps in parallel. Two pumps are normally operating and the remaining pump is available for immediate startup in the event of failure of a running pump. The cooling loop has two $50 \%$ capacity heat exchangers in parallel. The primary and secondary working fluids of the blanket coolant system are light water. The pumps are a canned-motor type.

The two RHR loops are both sized such that each can accommodate $100 \%$ of the decay heat removal requirements. Currently, the RHR components are sized for approximately $4 \%$ of the full power flow, $1569 \mathrm{~kg} / \mathrm{sec}$. Each of the RHR loops has one heat exchanger and one pump. The RHR loops may be isolated from the blanket primary loop with valves. The current baseline design has the RHR systems shutdown in standby mode during normal operation to enhance the system's response to some abnormal accident scenarios. This approach is compatible with the natural circulation capability of the RHR loops. The two RHR secondary side loops are also sized for each to accommodate $100 \%$ of the decay heat removal requirements. The RHR primary side and secondary side pumps are supplied with on-site backup diesel power as well as a dc backup power supply. Both the primary HR and the RHR loops are connected to the large fixed headers (20.5 inch diameter) that are internal to the target/blanket cavity vessel. The blanket heat energy is transported by the primary HR or RHR coolant water to the heat exchangers, and is transferred to the secondary side of the heat exchanger through the multiple tube shells. Schematic diagrams of the primary HR and RHR systems are shown in Sections 3 and 5. Detailed TRAC modeling descriptions for the primary HR and RHR systems are provided in the previous sections. This section describes the TRAC modeling of the secondary sides of HR and RHR heat exchangers.

\subsection{1-D TRAC Model for HR and RHR Secondary Sides}

The blanket heat removal system heat exchangers are conventional shell and tube type, horizontal heat exchangers, with single-pass or two-pass configurations. The HR cooling system has two-pass horizontal heat exchangers, and a single-pass horizontal heat exchanger is used in the RHR system. The current TRAC model does not include the entire secondary loops of the HR and RHR systems. Operating conditions of the secondary systems are simulated by using fill and break boundary conditions at the inlets and outlets of the secondary sides of heat exchanger components. A simple energy balance approach was taken to determine flow inputs for the secondary side of the heat exchanger in the one-dimensional TRAC system model. The basic approach to determine the secondary flow conditions is shown in Fig. 7.2-1. According to the 
current CDR, heat exchanger exit temperatures for the primary coolant are about $50 \mathrm{C}$ under normal operation conditions.

From asteady-state thermal energy balance,

$$
\mathrm{Q} \approx \mathrm{M}_{\mathrm{sf}} \mathrm{C}_{\mathrm{p}}\left(\mathrm{T}_{\mathrm{sfo}}-\mathrm{T}_{\mathrm{sfi}}\right) \approx \mathrm{M}_{\mathrm{pf}} \mathrm{C}_{\mathrm{p}}\left(\mathrm{T}_{\mathrm{pfi}}-\mathrm{T}_{\mathrm{pfo}}\right)
$$

where

$$
\begin{array}{ll}
M_{p f} & =\text { primary side liquid flowrate } \\
\mathrm{C}_{\mathrm{p}} & =\text { coolant specific heat } \\
\mathrm{T}_{\mathrm{pfo}} & =\text { primary coolant outlet temperature } \\
\mathrm{T}_{\mathrm{pfi}} & \text { = primary coolant inlet temperature } \\
\mathrm{M}_{\mathrm{sf}} & =\text { secondary side liquid flowrate } \\
\mathrm{T}_{\text {sfo }} & =\text { secondary coolant outlet temperature } \\
\mathrm{T}_{\mathrm{sfi}} & =\text { secondary coolant inlet temperature }
\end{array}
$$

From eq. 7.2.1, secondary coolant flow of a HR heat exchanger is about $1570 \mathrm{~kg} / \mathrm{sec}$. In a similar fashion, the flowrate, to provide the fill boundary condition for the secondary side inlet of the RHR heat exchanger, was calculated. Geometric dimensions and onedimensional lumped TRAC model components for the counter-current flow heat exchanger are shown in Fig. 7.2-2. Figure 7.2-3 shows heat exchanger fluid and solid heat structure nodalizations to model the secondary side of the heat exchanger.

\subsection{References}

1. Safety Code Development Group, "TRAC-PF1/MOD2: An Advanced Best Estimate Computer Program for Pressurized Water Reactor Thermal-Hydraulic Analysis," Los Alamos National Laboratory report LA-12031-M, Vol. 1 (NUREG/CR-5673), (July 21, 1993). 


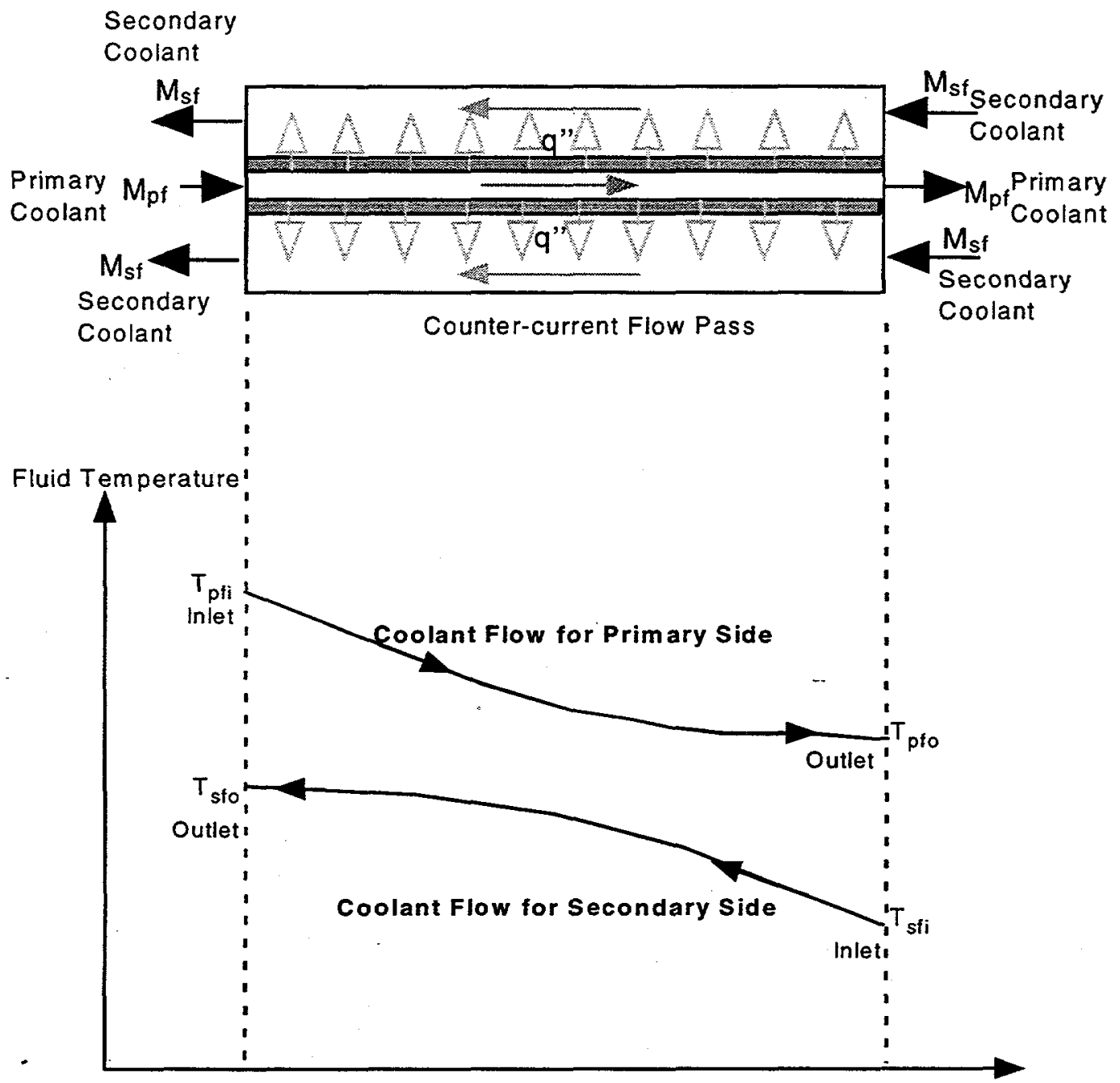

Figure 7.2-1 Typical coolant temperature profiles for primary and secondary heat exchanger sides 


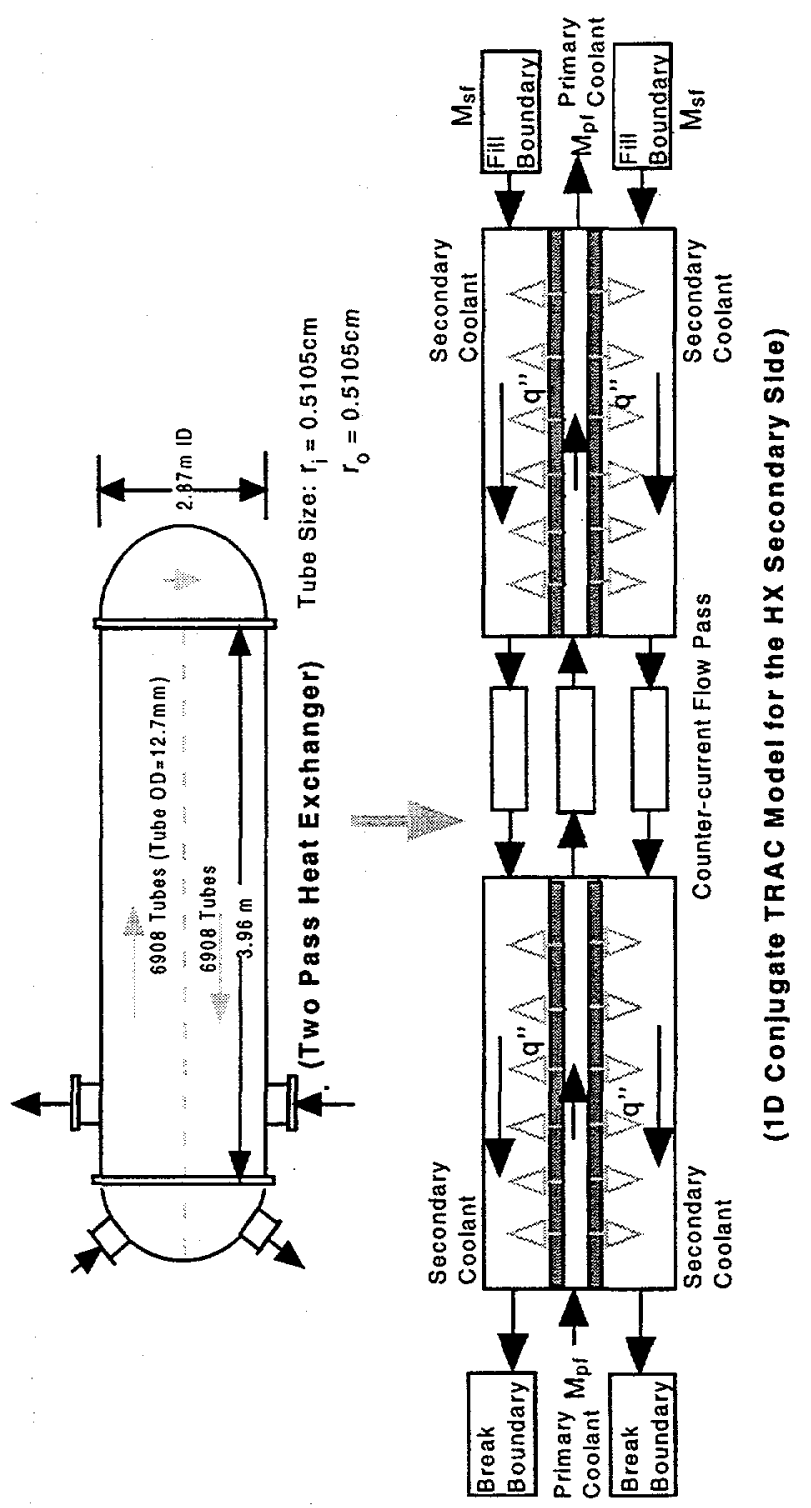

Figure 7.2-2 1-D TRAC model for the secondary side of primary HR heat exchanger. 

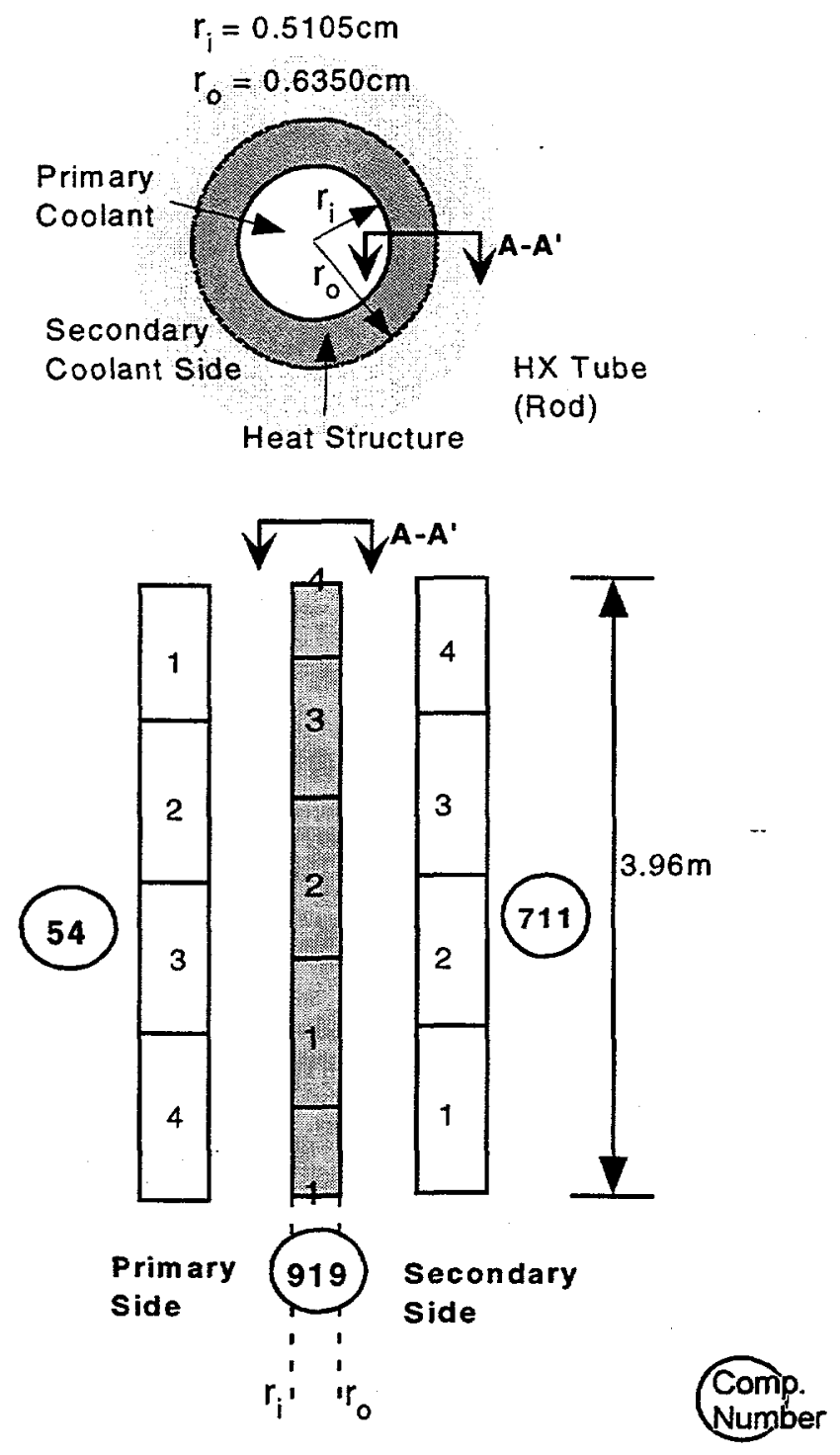

Secondary Side Noding Noding Under HX Flow Condition

Figure 7.2-3 Heat exchanger fluid and heat structure nodalization for 1-D TRAC model under counter-current flow pass. 


\section{Control Procedure Using Trip Signals, Signal Variables, and Control Valves}

Signal input parameters and procedures are used to control the blanket system operation in the simulation of LOCA transients. For the APT blanket system simulation control signal variables, control blocks, trips, and component action tables are defined (along with a multi-pass procedure) at the start of each code simulation. Values of the control signal variables are checked each time step. Component action tables list a component response as a function of elapsed time after actuation. Signal variables are independent variable values that are defined by action tables. The component action tables are parts of the component input. Figure 8-1 shows the control signal procedure to simulate a response to accident conditions and system component actions such as check valve control, heat structure power shutdown, and pump trips.

Normal operation conditions were simulated by using constrained steady-state (CSS) controllers or running the code in the transient mode. Pre-incident steady-state normal operation (NO) conditions of the APT blanket system were attained by specifying the desired total liquid flowrate of the two primary heat removal (HR) pumps with the CSS controller. In the TRAC model, 12 signal variables and 10 trip signals are used to control the APT blanket HR and RHR systems operation. Table 8-1 lists all of the signal variables used in the APT blanket system model. For the loss-of-flow accident (LOFA) and LOCA simulations, the transient values of: elapsed time, pressurizer pressure, open areas of check valves, and residual heat removal (RHR) pump speed are provided as transient boundary conditions. The heat structure power trip is controlled by using the pressure signal of the pressurizer surge line and a specified time delay. To avoid flow reversals, the opening and closing of check valves, at the discharge sides of HR and RHR pumps, was controlled by using component action data tables with S-shaped forcing functions. Figure 8-2 shows a S-shape forcing function curve. Table 8-2 shows all the trip signals used in the present blanket system model. The accident analysis methodology calls for several design basis accident scenarios, including, LOFA and internal/external large break LOCA's, and NO to be simulated with the TRAC system model to provide local boundary conditions for the detailed FLOWTRAN-TF bin model. The accident scenarios were simulated by using control signal variables. The initial conditions and values of the signal variables are listed in Table 8-3.

\subsection{References}

1. Safety Code Development Group, "TRAC-PF1/MOD2: An Advanced Best Estimate Computer Program for Pressurized Water Reactor Thermal-Hydraulic Analysis," Los Alamos National Laboratory report LA-12031-M, Vol. 1 (NUREG/CR-5673), (July 21, 1993). 
TRAC BLANKET SYSTEM MODEL

Table 8-1 Control signal variables used in the APT blanket system model.

\begin{tabular}{|c|c|c|c|}
\hline $\begin{array}{l}\text { Signal Variable } \\
\text { I No. }\end{array}$ & Control Parameter & System Component & Descriptions \\
\hline 1 & time & $-\cdots$ & delay time \\
\hline 2 & pressure difference & RHR check valve & no flow reversal \\
\hline 3 & pump speed and time & $\begin{array}{l}\text { RHR pump and cavity } \\
\text { vessel vent valve }\end{array}$ & $\begin{array}{l}\text { S-shaped forcing function } \\
\text { using action table }\end{array}$ \\
\hline 4 & -- & HR pump & not used \\
\hline 5 & pressure difference & $\begin{array}{l}\text { HR check valve for } \\
\text { pump\#1 }\end{array}$ & $\begin{array}{c}\text { S-shaped forcing function } \\
\text { using action table }\end{array}$ \\
\hline 6 & pressure difference & $\begin{array}{l}\text { HR check valve for } \\
\text { pump\#2 }\end{array}$ & $\begin{array}{l}\text { S-shaped forcing function } \\
\text { using action table }\end{array}$ \\
\hline 7 & pressure & pressurizer & pressurizer pressure \\
\hline 8 & time & $\begin{array}{l}\text { module valve for internal } \\
\text { LOCA simulation }\end{array}$ & $\begin{array}{l}\text { S-shaped forcing function } \\
\text { using action table }\end{array}$ \\
\hline 9 & time & $\begin{array}{l}\text { HR valve for internal } \\
\text { LOCA simulation }\end{array}$ & elapsed time \\
\hline 10 & pressure & cavity flooding system & pressure \\
\hline 11 & -- & cavity flood system & not used \\
\hline 12 & time & cavity flood line valve & $\begin{array}{l}\text { S-shaped forcing function } \\
\text { using action table }\end{array}$ \\
\hline
\end{tabular}

Table 8-2 Trip signal variables used in the APT blanket system model.

\begin{tabular}{|c|c|c|c|}
\hline $\begin{array}{l}\text { Trip Signal } \\
\text { ID Number }\end{array}$ & Control Component & $\begin{array}{l}\text { Signal Variables } \\
\text { 10 Number (Control } \\
\text { Variable) }\end{array}$ & $\begin{array}{c}\text { Signal Value } \\
\text { under No }\end{array}$ \\
\hline 101 & $\begin{array}{l}\text { Fill boundary for } \\
\text { secondary heat } \\
\text { exchanger side }\end{array}$ & 1 (time) & $1.0 \mathrm{e}-06 \mathrm{sec}$ \\
\hline 102 & Cavity flood system valve & 10 (cavity vessel pressure) & valve closed \\
\hline 103 & RHR check valve & 1 (time) & $1.0 \mathrm{e}-06 \mathrm{sec}$ \\
\hline 104 & Heat structure & $\begin{array}{c}7 \text { (pressurizer pressure) } \\
\text { and time delay }\end{array}$ & no trips \\
\hline 105 & RHR pump & $\begin{array}{c}7 \text { (pressurizer pressure) } \\
\text { and RHR } \Delta \mathrm{P}\end{array}$ & off \\
\hline 106 & Primary HR pump & $\begin{array}{c}7 \text { (pressurizer pressure) } \\
\text { and time delay }\end{array}$ & on \\
\hline 107 & Primary HR check valve & $\begin{array}{l}1 \text { (time) and action table (S- } \\
\text { shaped forcing function) }\end{array}$ & $1.0 \mathrm{e}-08 \mathrm{sec}$ \\
\hline 108 & Cavity vessel check valve & 1 (time) & valve closed \\
\hline 109 & $\begin{array}{c}\text { Cavity vessel check valve } \\
\text { on the HR side }\end{array}$ & 1 (time) & valve closed \\
\hline 110 & Cavity vessel vent valve & 7 (pressurizer pressure) & valve closed \\
\hline
\end{tabular}


TRAC BLANKET SYSTEM MODEL

Page:

Table 8-3 Design basis accident scenarios for the APT blanket system safety analysis.

\begin{tabular}{|c|c|c|}
\hline \multicolumn{2}{|c|}{ Accident Scenarios } & Initial and System Control Signal Conditions \\
\hline \multicolumn{2}{|c|}{ Pre-Accident Normal Operation } & $\begin{array}{l}\text { Steady state normal operation conditions } \\
\text { Total liquid flowrate: } 1569 \mathrm{~kg} / \mathrm{sec} \\
\text { Total power: } 56.5 \mathrm{MW}\end{array}$ \\
\hline \multicolumn{2}{|r|}{ Pump Coastdown } & $\begin{array}{l}\text { Beam trip: } 1.0 \text { sec delay after } 5 \% \text { reduction of } \\
\text { Pzr surge line pressure } \\
\text { RHR pump trip: signal activated after } 5 \% \\
\text { reduction of pump } \Delta P\end{array}$ \\
\hline \multirow[t]{5}{*}{$\begin{array}{l}\text { External } \\
\text { LOCA }\end{array}$} & $\begin{array}{l}\text { Case 1: HR Cold Leg Break } \\
\text { (Break Location: Comp. } 65 \text { ) }\end{array}$ & $\begin{array}{l}\text { Pump trip: } 0.2 \text { sec delay after } 5 \% \text { reduction of } \\
\text { Pzr surge line pressure } \\
\text { Beam trip: } 0.2 \text { sec delay after } 5 \% \text { reduction of } \\
\text { Pzr surge line pressure } \\
\text { RHR pump: signal activated after } 5 \% \text { reduction } \\
\text { of Pzr surge line pressure }\end{array}$ \\
\hline & $\begin{array}{l}\text { Case 2: Pump Discharge } \\
\text { Side } \\
\text { Break with Pump Trip } \\
\text { (Break Location: Comp. 37) }\end{array}$ & $\begin{array}{l}\text { Pump trip: } 0.2 \text { sec delay after } 5 \% \text { reduction of } \\
\text { Pzr surge line pressure } \\
\text { Beam trip: } 0.2 \text { sec delay after } 5 \% \text { reduction of } \\
\text { Pzr surge line pressure } \\
\text { RHR pump: signal activated after } 5 \% \text { reduction } \\
\text { of Pzr surge line pressure }\end{array}$ \\
\hline & $\begin{array}{l}\text { Case 3: Pump Discharge } \\
\text { Side Break without Pump } \\
\text { Trip } \\
\text { (Break Location: Comp. 37) }\end{array}$ & $\begin{array}{l}\text { Pump trip: no pump trip } \\
0.2 \text { sec delay after } 5 \% \text { reduction of Pzr surge } \\
\text { line pressure } \\
\text { RHR pump: signal activated after } 5 \% \text { reduction } \\
\text { of Pzr surge line pressure }\end{array}$ \\
\hline & $\begin{array}{l}\text { Case 4: Pzr Surge Line } \\
\text { Break } \\
\text { (Break Location: Comp. } \\
764 \text { ) }\end{array}$ & $\begin{array}{l}\text { Pump trip: } 0.2 \text { sec delay after } 5 \% \text { reduction of } \\
\text { Pzr surge line pressure } \\
\text { Beam trip: } 0.2 \text { sec delay after } 5 \% \text { reduction of } \\
\text { Pzr surge line pressure } \\
\text { RHR pump: signal activated after } 5 \% \text { reduction } \\
\text { of Pzr surge line pressure }\end{array}$ \\
\hline & $\begin{array}{l}\text { Case 5: RHR Cold Leg } \\
\text { Break } \\
\text { (Break Location: Comp. } \\
661 \text { ) }\end{array}$ & $\begin{array}{l}\text { Pump trip: } 0.2 \text { sec delay after } 5 \% \text { reduction of } \\
\text { Pzr surge line pressure } \\
\text { Beam trip: } 0.2 \text { sec delay after } 5 \% \text { reduction of } \\
\text { Pzr surge line pressure } \\
\text { RHR pump: signal activated after } 5 \% \text { reduction } \\
\text { of Pzr surge line pressure }\end{array}$ \\
\hline $\begin{array}{l}\text { Internal } \\
\text { LOCA }\end{array}$ & $\begin{array}{l}\text { Case 1: HR Cold Leg Break } \\
\text { (Break Location: Comp. 67- } \\
69 \text { ) } \\
\text { Case 2: Module } 1 \text { Inlet } \\
\text { Break (Break Location: } \\
\text { Comp. 80) }\end{array}$ & $\begin{array}{l}\text { Pump trip: } 0.2 \text { sec delay after } 5 \% \text { reduction of } \\
\text { Pzr surge line pressure } \\
\text { Beam trip: } 0.2 \text { sec delay after } 5 \% \text { reduction of } \\
\text { Pzr surge line pressure } \\
\text { RHR pump: signal activated after } 5 \% \text { reduction } \\
\text { of Pzr surge line pressure } \\
\text { Cavity valve actuated when liquid level (on cavity } \\
\text { vessel floor) }=1.5 \mathrm{~m} \text { (Comp. } 823 \text { ) } \\
\text { Cavity vessel vent valve actuated similar to heat } \\
\text { structures similar to heat structure without delay }\end{array}$ \\
\hline
\end{tabular}




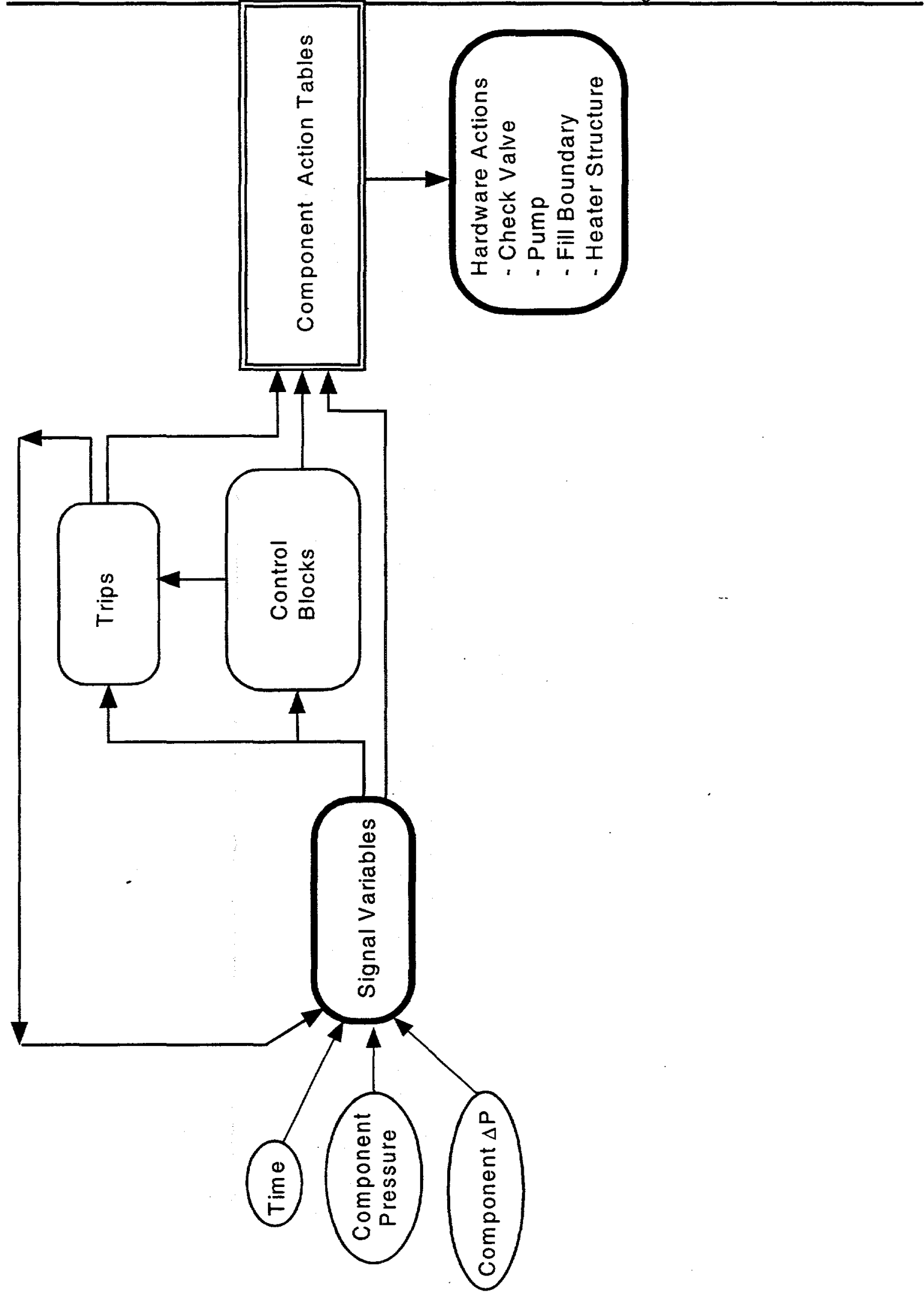

Figure 8-1 Control procedure for blanket system component hardware actions. 


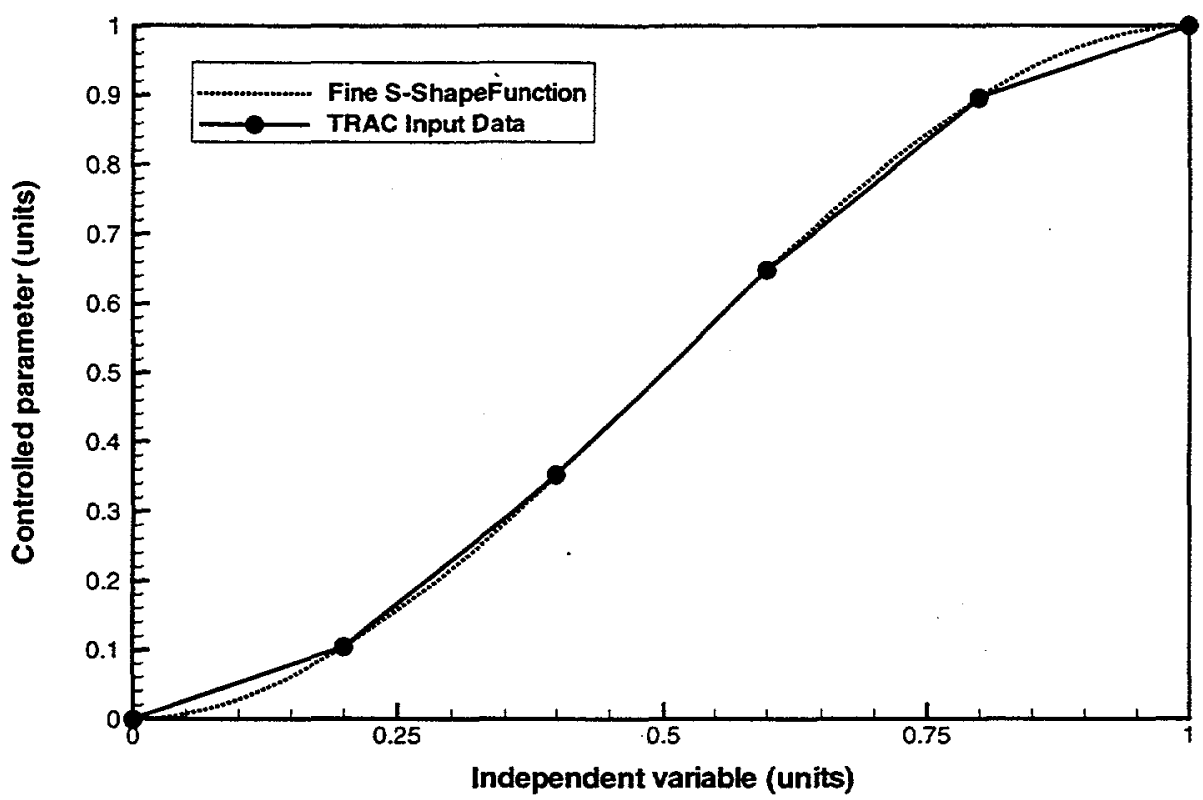

Figure 8-2 S-shape forcing function curve used in the present analysis. 


\section{Appendix A: TRAC Standard Input File for Normal Operation Case}

The file listed below represents the entire TRAC code "tracin" file that corresponds to the normal operation case for the blanket system, all of its primary heat removal (HR) and residual heat removal (RHR) piping components, its six lumped modules, the cavity vessel, and the cavity flood system. This input deck provides the nominal design basis case for normal operation (NO) and the TRAC restart file ("trcrst") for all design basis accidents (DBAs) considered.

\section{Input file tracin:}

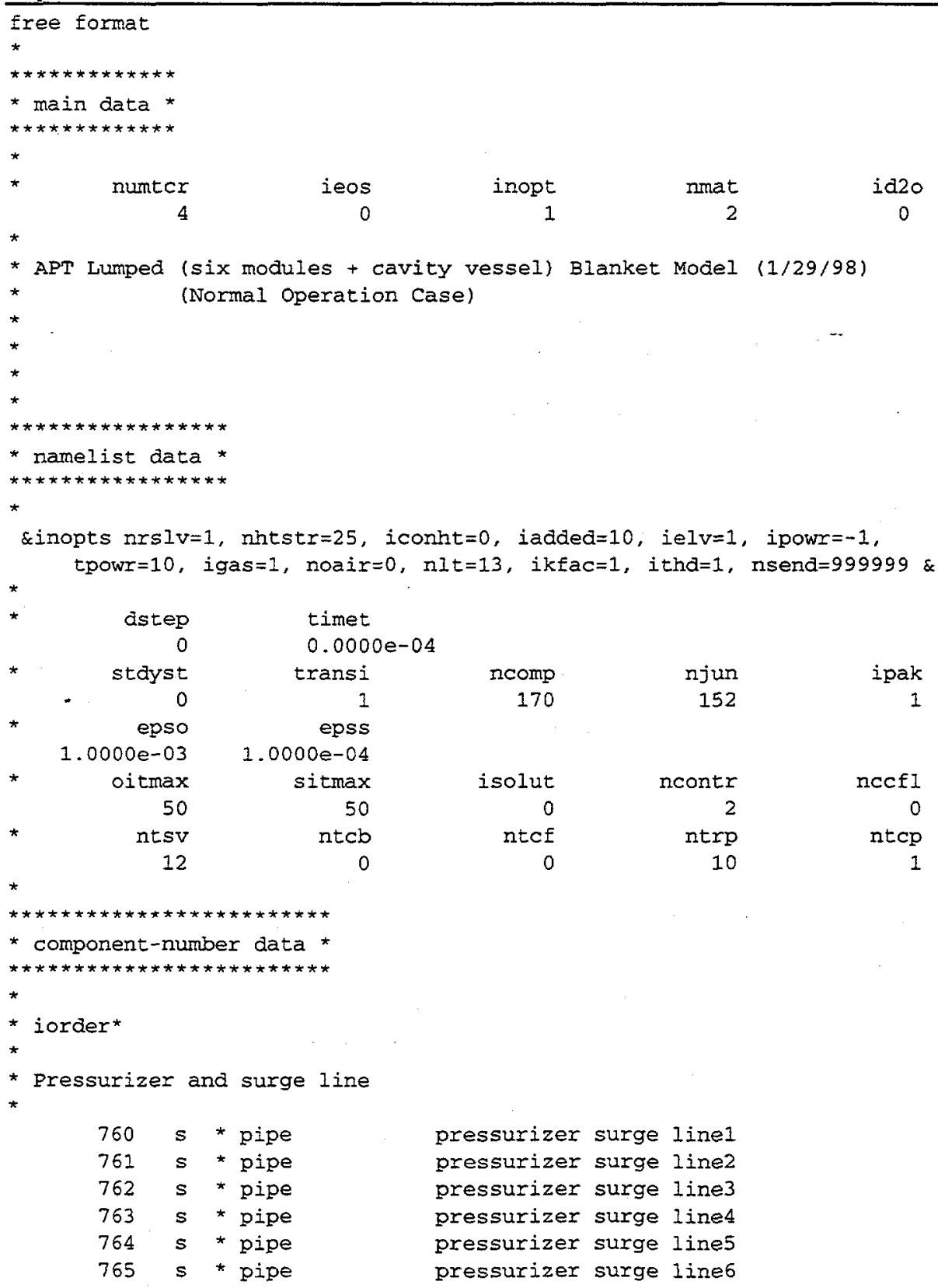




766 s * pipe

767 s * break

* HR hot leg

\begin{tabular}{|c|c|c|}
\hline 20 & $s$ & * pipe \\
\hline 21 & $s$ & * plenum \\
\hline 22 & $s$ & * pipe \\
\hline 23 & $s$ & * pipe \\
\hline 24 & $\mathrm{~s}$ & * pipe \\
\hline 25 & $s$ & * pipe \\
\hline 26 & $s$ & ipe \\
\hline
\end{tabular}

* HR pumps

\begin{tabular}{|c|c|c|}
\hline 27 & $s$ & * plenum \\
\hline 28 & $s$ & * pipe \\
\hline 30 & $s$ & * pump \\
\hline 32 & $s$ & * valve \\
\hline 29 & $s$ & * pipe \\
\hline 31 & $s$ & * pump \\
\hline 33 & $s$ & * valve \\
\hline 34 & $s$ & * plenum \\
\hline
\end{tabular}

* HR pump-to-hx piping

$\begin{array}{ll}36 & s \\ 37 & s \text { * pipe } \\ 38 & s \text { * pipe }\end{array}$

* HR hx's

\begin{tabular}{|c|c|c|}
\hline 40 & $s$ & * plenum \\
\hline 48 & $s$ & * pipe \\
\hline 50 & $\mathrm{~s}$ & * pipe \\
\hline 52 & $s$ & * pipe \\
\hline 54 & $\mathbf{s}$ & * pipe \\
\hline 56 & $s$ & * pipe \\
\hline 49 & $s$ & * pipe \\
\hline 51 & $s$ & * pipe \\
\hline 53 & $s$ & * pipe \\
\hline 55 & $s$ & * pipe \\
\hline 57 & $s$ & * pipe \\
\hline 60 & $\mathrm{~s}$ & * plenum \\
\hline
\end{tabular}

* hr cola leg

$\begin{array}{rrl}62 & s & \text { * pipe } \\ 63 & \mathrm{~s} & \text { * pipe } \\ 64 & -\mathrm{s} & \text { * pipe } \\ 65 & \mathrm{~s} & \text { * pipe } \\ 66 & \mathrm{~s} & \text { * pipe } \\ 67 & \mathrm{~s} & \text { * plenum } \\ 854 & \mathrm{~s} & \text { * valve } \\ 69 & \mathrm{~s} & \text { * plenum } \\ 68 & \mathrm{~s} & \text { * pipe }\end{array}$

* HR hx Secondary Side

\begin{tabular}{|c|c|c|}
\hline 710 & $s$ & \\
\hline 711 & $s$ & \\
\hline 712 & $s$ & \\
\hline 713 & $\mathbf{s}$ & \\
\hline 714 & $s$ & \\
\hline 730 & $\mathbf{s}$ & \\
\hline 731 & $s$ & \\
\hline 732 & $\mathrm{~s}$ & \\
\hline 733 & $\mathbf{s}$ & \\
\hline 734 & $\mathbf{s}$ & \\
\hline
\end{tabular}

* RHR primary loop

$$
621 \mathrm{~s} \text { * pipe }
$$

primary pressurizer

pressurizer boundary

HR pump suction pipe

HR pump suction pipe (bk)

HR pump suction pipe

HR purmp suction pipe (bk)

HR pump suction pipe

HR pump suction pipe (bk)

$H R$ pump suction pipe

HR pump suction plenum

HR pump \#1 inlet pipe

HR pump \#1

HR pump \#1 outlet pipe

HR pump \#2 inlet pipe

HR pump \#2

HR pump \#2 outlet pipe

HR pump discharge plenum

HR pump dicharge pipe HR pump dicharge pipe (bk) HR pump dicharge pipe

HR hx inlet plemum $\mathrm{HR}$ hx 1 inlet pipe HR hx 1 tubes 1st pass HR hx 1 mid-header HR hx 1 tubes 2nd pass HR $h x 1$ outlet pipe $H R$ hx 2 inlet pipe HR hx 2 tubes 1st pass HR hx 2 mid-header HR hx 2 tubes 2nd pass HR hx 2 outlet pipe HR hx outlet plenum

HR hx discharge pipe HR $h x$ discharge pipe (bk) HR hx discharge pipe HR $\mathrm{hx}$ discharge pipe (bk) HR $h x$ discharge pipe HR hx discharge pipe (bk) $H R$ hx discharge pipe (bk) HR hx discharge pipe (bk) HR $\mathrm{hx}$ discharge pipe

HR hx Secondary Side-1 HR hx Secondary side-1 HR hx Secondary Side-1 HR hx Secondary side-1 HR hx Secondary Side-1 HR hx Secondary Side-2 HR hx Secondary Side-2 HR hx Secondary Side-2 HR hx Secondary Side-2 HR $\mathrm{hx}$ Secondary Side-2

\author{
) \\ ) \\ (n)
}




\begin{tabular}{|c|c|c|}
\hline 623 & $s$ & * pipe \\
\hline 624 & $s$ & * pipe \\
\hline 625 & $\mathrm{~s}$ & * pipe \\
\hline 630 & s & * pump \\
\hline 640 & $s$ & alve \\
\hline 652 & $s$ & ipe \\
\hline 660 & $s$ & * pipe \\
\hline 661 & $s$ & * pipe \\
\hline 662 & s & * pipe \\
\hline 663 & $\mathrm{~s}$ & * pipe \\
\hline
\end{tabular}

* RHR hx Secondary Side

$672 \mathrm{~s}$ * $\mathrm{f}$ iII

$671 s$ * pipe

673 s * break

*

\begin{tabular}{|c|c|c|}
\hline 30 & $s$ & * plenum \\
\hline 32 & $s$ & * pipe \\
\hline & $\mathbf{s}$ & plenum \\
\hline & s & * pipe \\
\hline 15 & 5 & * plenum \\
\hline 38 & $\mathbf{s}$ & * pipe \\
\hline 87 & & Lenum \\
\hline 90 & 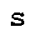 & * pipe \\
\hline 89 & $s$ & Lenun \\
\hline 84 & $\mathrm{~s}$ & * \\
\hline & & 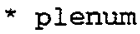 \\
\hline
\end{tabular}

$300 s *$ pipe

$330 \mathrm{~s}$ * plenum

335 s * pipe

340 s * plenum

$350 \mathrm{~s} *$ plenum

$360 \mathrm{~s} *$ pipe

$370 \mathrm{~s}$ * plenum

375 s * pipe

$380 \mathrm{~s} *$ plenum

429 s * pipe

$454 \mathrm{~s}$ * pipe

173 s * pipe

- $172 \mathrm{~s} *$ plemum

158 s * pipe

$147 \mathrm{~s}$ * plenum

102 s * pipe

$133 \mathrm{~s}$ * plenum

136 s * pipe

541 s * pipe

$538 \mathrm{~s}$ * plenum

535 s * pipe

531 s * plenum

528 s * pipe

536 s * plenum

$539 \mathrm{~s}$ * pipe

415

479

478

418

409

423

417
RHR hot leg sect 2

RHR hot leg sect 3 (bk)

RHR hot leg sect 4

RHR primary pump

RHR pump discharge valve

RHR primary heat exchanger tubes

RHR cold leg sect 1

RHR cold leg sect 2 (bk)

RHR cold leg sect 3

RHR cold leg sect 4 (bk)

RHR hx Secondary Side

RHR hx Secondary Side

RHR hx Secondary Side

L1 Blanket Row1 Plenum

L2 Blanket Row1

I2 Blanket Row1 Plenum

L3 Blanket Row1

I3 Blanket Row1 Plenum

I4 Blanket Row1

I4 Blanket Row I Plenum

I5 Blanket Row1

L5 Blanket Row1 Plenum

L6 Blanket Row I

I6 Blanket Row1 Plenum

L1 Blanket Row1

L1 Blanket Rowl Plenum

I1 pipe conn. $330-340$

L1 outlet header

L1 lower plenum

L1 decoupler

L1 decoupler upper plenum

L1 pipe conn. $370-380$

Ll inlet header

L1 connect hot header-tee

LI connect cold header-tee

L1 Blanket Row1

L1 Blanket Row1 Plenum

L1 pipe conn. $330-340$

L1 outlet header

L1 lower plenum

Il decoupler

Il decoupler upper plenum

L1 Blanket Row1

Ll Blanket Row1 Plenum

L1 pipe conn. $330-340$

L1 outlet header

L1 lower plenum

LI decoupler

II decoupler upper plenum

L1 Blanket Row1

LI Blanket RowI Plenum

L1 pipe conn. $330-340$

Ll outlet header

L1 lower plenum

II decoupler

LI decoupler upper plenum 


\begin{tabular}{|c|c|c|}
\hline 185 & s & * pipe \\
\hline 489 & $s$ & * plenum \\
\hline 80 & $s$ & * pipe \\
\hline & $s$ & * plenum \\
\hline & $s$ & * pipe \\
\hline & s & * plenum \\
\hline & $s$ & * pipe \\
\hline & $s$ & * pipe \\
\hline 10 & $s$ & * plenum \\
\hline & $s$ & * pipe \\
\hline & $s$ & * plenum \\
\hline & $s$ & ipe \\
\hline & $s$ & lenum \\
\hline & $\mathrm{s}$ & ipe \\
\hline
\end{tabular}

* Cavity Pool

\begin{tabular}{|c|c|c|}
\hline 801 & $\mathrm{~s}$ & * break \\
\hline 810 & $s$ & * pipe \\
\hline 811 & s & * plenum \\
\hline 812 & $s$ & * pipe \\
\hline 813 & $s$ & * plenum \\
\hline
\end{tabular}

* Cavity Flood Iine

$820^{\circ} \mathrm{s}$ * valve
821 s * pipe
822 s * pipe

* Cavity vessel

\begin{tabular}{|c|c|c|}
\hline 823 & $\mathbf{s}$ & * pipe \\
\hline 824 & $s$ & * plenum \\
\hline 825 & $s$ & * pipe \\
\hline 826 & $\mathbf{s}$ & * plenum \\
\hline
\end{tabular}

* Cavity Vessel -to- Primary Loops (HR \& Module lines)

Cavity pool bk BC

Cavity pool upper section

Cavity pool middle section

Cavity pool lower section

Cavity flood line valve

Cavity vessel lower section

Cavity pool bottom (no fill)

Cavity flood line external to vessel

Cavity flood line internal to vessel

Cavity vessel lower break location

Cavity vessel midale section

Cavity vessel upper break location

Cavity vessel to "Hot" Module inlet line

Cavity vessel to $\mathrm{HR}$ inlet (HR side)

Cavity vessel to $\mathrm{HR}$ inlet (Header side)

* Cavity Vent Iine

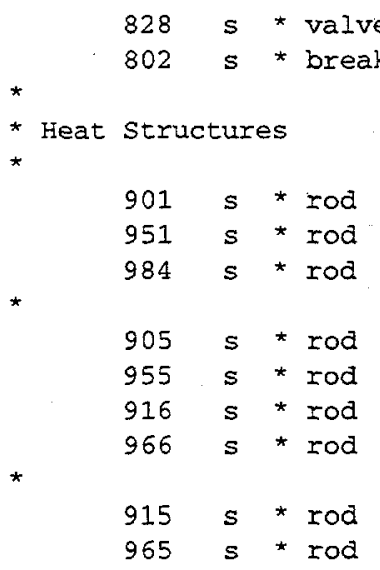

Cavity vessel vent line valve Cavity vent line bk BC

annular aluminum rod cylindrical lead rod cylindrical lead rod

annular aluminum rod cylindrical lead rod cylindrical lead rod cylindrical lead rod

annular aluminum rod cylindrical lead rod 

*

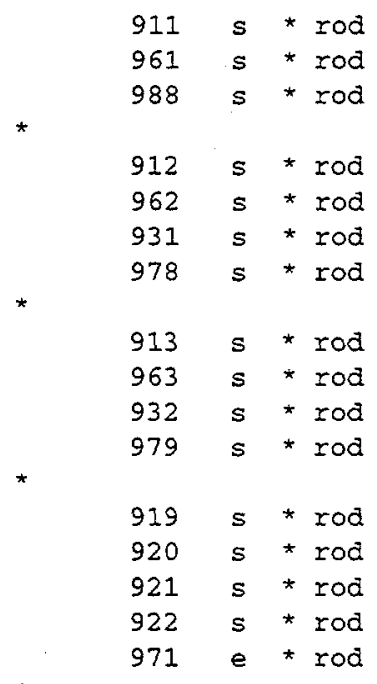

* material-properties data *

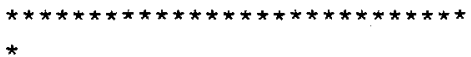

*

* matb *

* pebla *

\section{1}

* lead material

* $\begin{array}{ll}\operatorname{prptb}(1, i) & \operatorname{prptb}(2, i) \\ 2.7300 e+02 & 1.1374 e+04\end{array}$ $1.0000 e+10 \quad 1.1374 e+04$

e

* aluminum material

$$
\text { * }
$$

prptb $(1, i)$
$2.7300 e+02$

$3.0000 e+02$

$3.7300 e+02$

$4.7300 e+02$

$6.7300 e+02$

$1.0000 e+10$

$\stackrel{+}{*}$

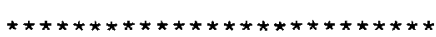

$*$

* cSS data

$\star$

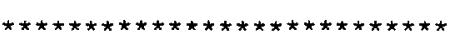

* numess amness

*

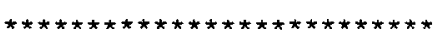

* control-parameter data *

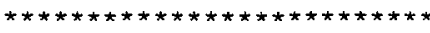

$\star$

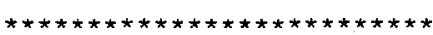

* signal variables

\begin{abstract}
annular aluminum rod cylindrical lead rod cylindrical lead rod
\end{abstract}

annular aluminum rod cylindrical lead rod cylinarical lead rod cylindrical lead rod

annular aluminum rod cylindrical lead rod cylindrical lead rod cylinarical lead rod

annular ss rod annular ss rod annular ss rod annular ss rod annular ss rod

$52 e$

$6 e$

$\operatorname{prptb}(3, i)$

$1.2970 \mathrm{e}+02$

prptb $(4, i)$

$3.4592 \mathrm{e}+01$

$3.3382 \mathrm{e}+01$

$\operatorname{prptb}(5, i)$

2. $8000 \mathrm{e}-01$

$2.8000 e-01$ $\operatorname{prptb}(3, i)$

$8.6985 e+02$

$8.9000 e+02$

$9.4140 \mathrm{e}+02$

$9.9538 e+02$

$1.0900 e+03$

$1.2000 \mathrm{e}+03$ $\operatorname{prptb}(4, i)$

$2.1046 e+02$

$2.1046 e+02$

$2.1046 \mathrm{e}+02$

2. $2175 e+02$

$2.2845 e+02$

$2.3000 e+02$ prptb $(5, i)$

$5.0000 e-02$

$5.0000 e-02$

$5.0000 e-02$

$5.0000 \mathrm{e}-02$

5.0000 e- 02

$5.0000 e-02$

amxcss

nmpess

napcss 
*

* time

* ids

idsv

isvn

ilcn

icn 1

icn 2

$\star$

* pressure difference across RHR check vaive

* idsv isvn ilcn

$2 \quad-21 \quad 640$

icn 1

icn 2

$\star$

* Elapse time since RHR pump activated

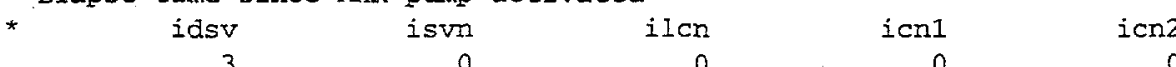

*

* Elapse time since HR pumps and cavity vessel vent valve activated

* idsv isvn ilcn icnl icn2

*

* pressure difference across HR check valve

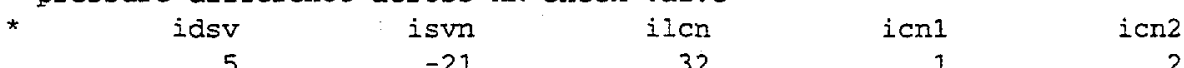

*

* pressure difference across HR check valve

* $\begin{array}{rrrrr}\text { idsv } & \text { isvn } & \text { ilcn } & \text { icn1 } & \text { icn2 }\end{array}$

* pressure, cold leg at plenum, component 761

* iasv ism $\quad$ ilcn icn1 icn2

*

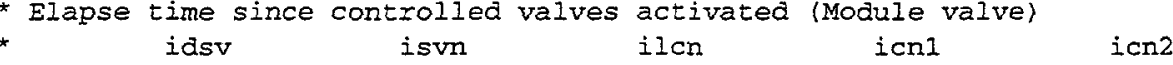

* Elapse time since controlled valves activated (HR valves)

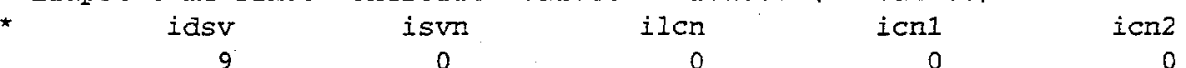

*

* Pressure of component 823 isva ilcn icn 1 icn
$10 \quad 21$
1
1

* Free slot

Free slot

idsv

isvo

ilen

$\operatorname{icn} 1$

icn2

*

* Elapse time since controlled valves activated (CFS valve)

* idsv isvn ilcn icn1 icn2

$\star$ 12

0

0

0

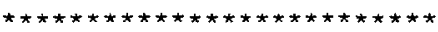

* control-block data *

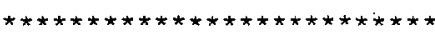

$\star$

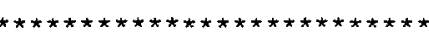

$\star$ trips

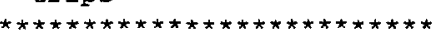

$*$

* trips from off to on at time given by setp(2), secondary HX's

$\begin{array}{rrrrr}\text { * ntse } & \text { ntct } & \text { ntsf } & \text { ntdp } & \text { ntsd } \\ \text { * } & 0 & 0 & 0 & 0 \\ \text { idtp } & \text { isrt } & \text { iset } & \text { itst } & \text { idsg } \\ 101 & 2 & 0 & 1 & 1\end{array}$




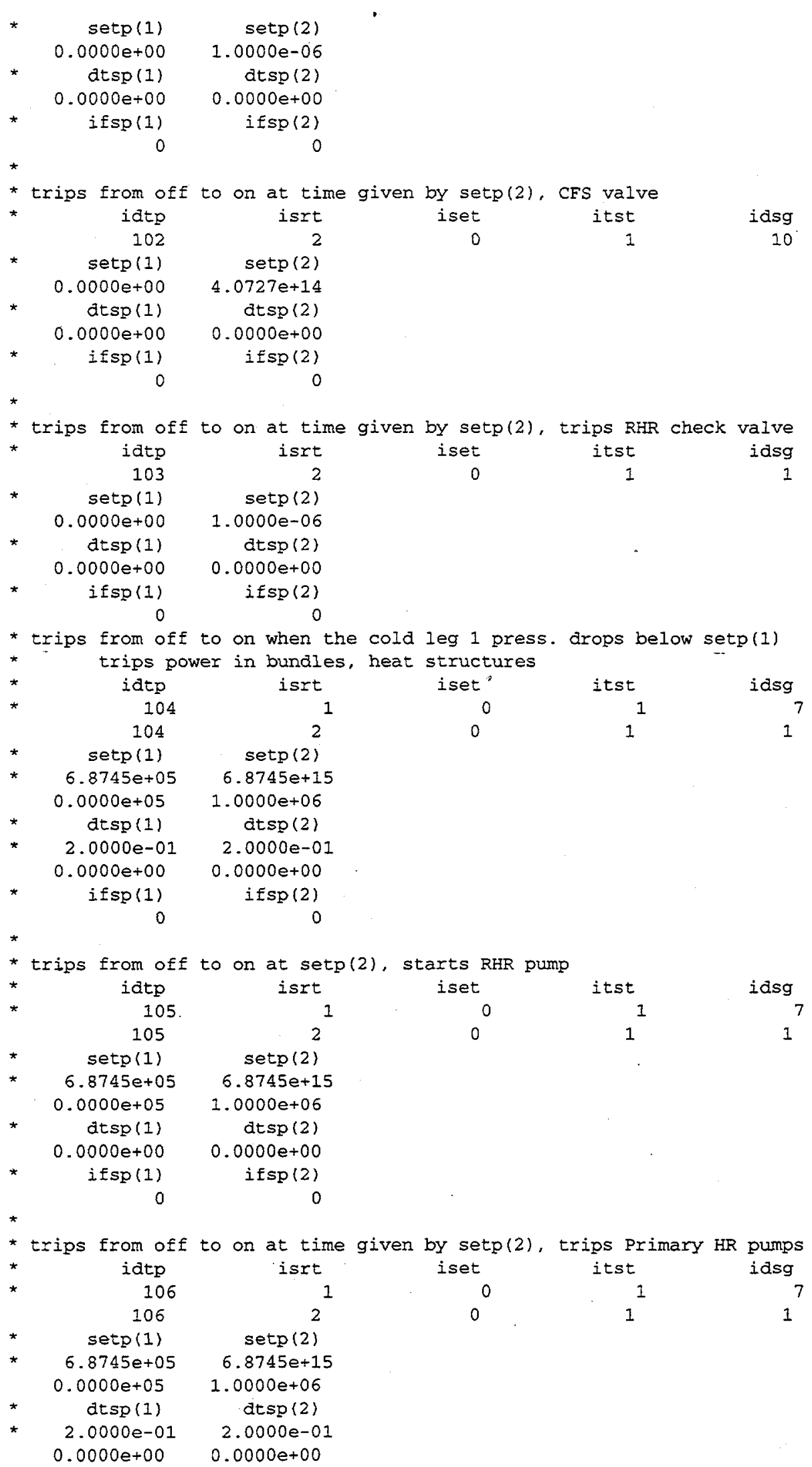




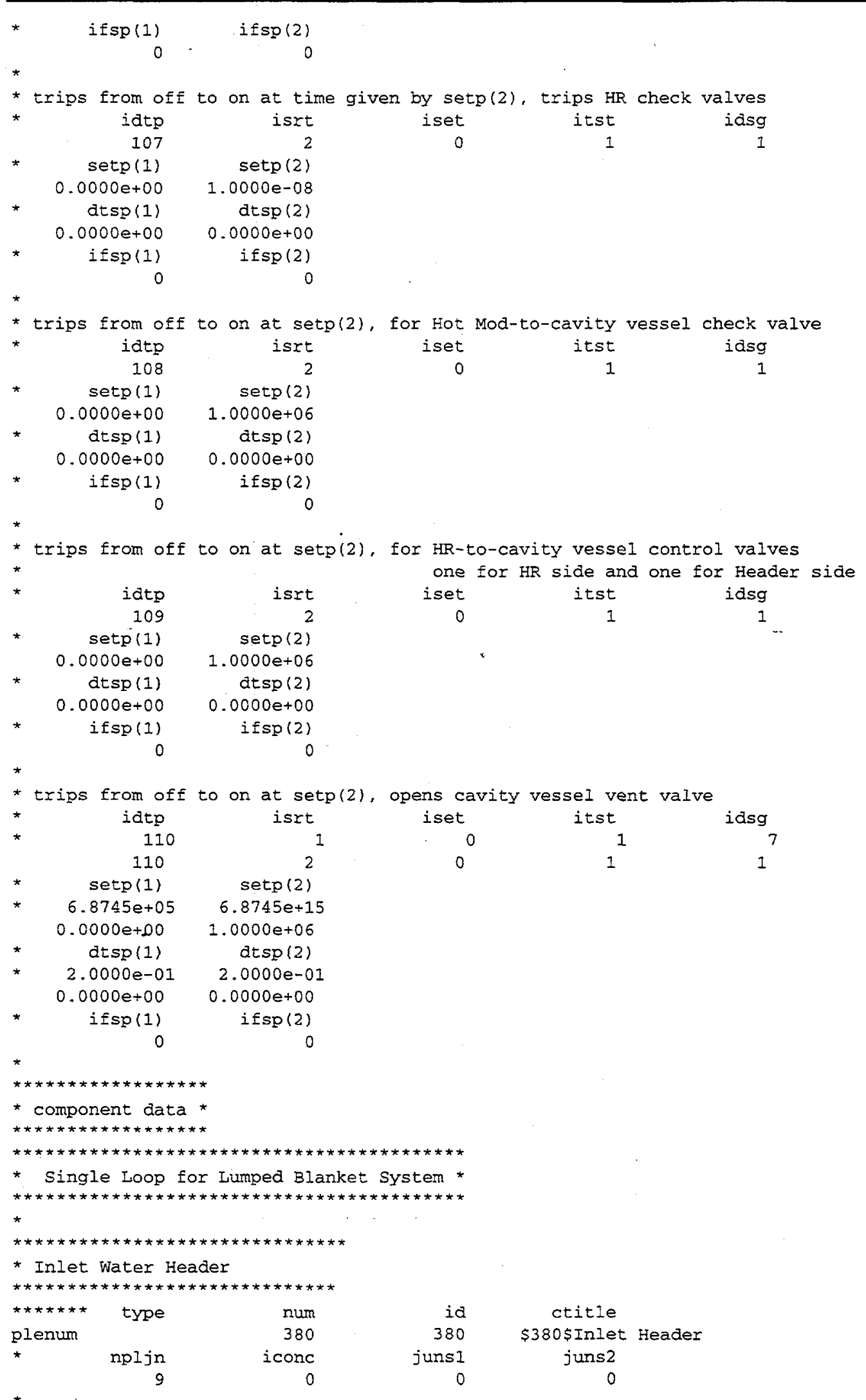


* juncs * $454 \mathrm{~s}$

* juncs * $173 \mathrm{~s}$

* juncs * 541s

* junes * $415 \mathrm{~s}$

* juncs * $485 \mathrm{~s}$

* juncs * $513 \mathrm{~s}$

* juncs * $760 \mathrm{~s}$

* juncs * $664 \mathrm{e}$

Connected to Primary Coolant Loop

*Connected to Ist Lateral Decoup upper PI

*Connected to 2nd Lateral Decoup Upper P1

*Connected to 3rd LoPwr Mod Decoup Upper Pl

*Connected to 1st DNS Mod Decoup Upper PI

*Connected to 2nd DNS Mod Decoup upper PI

*Connected To 3ra DNS Mod Decoup Upper P1

* Connected To Pressurizer

$* \mathrm{dx} \quad * \mathrm{f} \quad 5.2070 \mathrm{e}-01 \mathrm{e}$

* vol * $3.1727 \mathrm{e}-01 \mathrm{e}$

$*$ elev * $2.0375 e+00 e$

*alp * $\quad 0.0000 e+00 e$

$\star t 1 * 3.2315 \mathrm{e}+02 \mathrm{e}$

*tv * $\quad * 2315 \mathrm{e}+02 \mathrm{e}$

$* \mathrm{p} \quad * \quad 7.32556 \mathrm{e}+05 \mathrm{e}$

$* \mathrm{pa} * 0.0000 \mathrm{e}+00 \mathrm{e}$

* Outlet Header

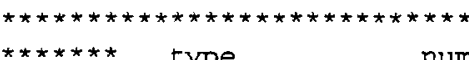

plenum $\quad 340$

id ctitle

* npljn iconc

$340 \$ 340 \$$ outlet Head

8

juns I

juns2

*

* juncs * 20

* juncs * 429s

* juncs * $127 s$

* juncs * $539 \mathrm{~s}$

* juncs * $417 \mathrm{~s}$

* juncs * $484 \mathrm{~s}$

* juncs * $511 s$

* juncs * $621 \mathrm{e}$

* Connected to Primary Coolant Loop

* Ist Lateral RI Pl to outlet Header

*2nd Lateral RI P1 to outlet Header

* Low Pwr R1 PI to Outlet Header

*1st DNS R1 PI to Outlet Header

*2nd DNS RI PI to outlet Header

*3rd DNS R1 PI to outlet Header

$* d x \quad * f \quad 5.2070 e-01 e$

* vol * $3.1727 \mathrm{e}-01 \mathrm{e}$

* elev * $1.2755 \mathrm{e}+00 \mathrm{e}$

*alp * $0.0000 e+00 e$

$* t$ t $* 3.3315 \mathrm{e}+02 \mathrm{e}$

*tv. * $\quad 3.3315 \mathrm{e}+02 \mathrm{e}$

* $\mathrm{p} \quad$ * 4.53187e+05e

$\star \mathrm{pa} * \quad 0.0000 \mathrm{e}+00 \mathrm{e}$

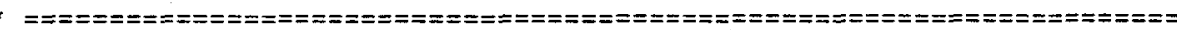

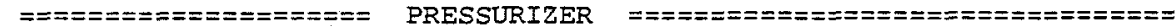

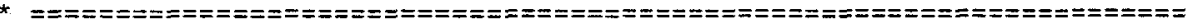

$*$

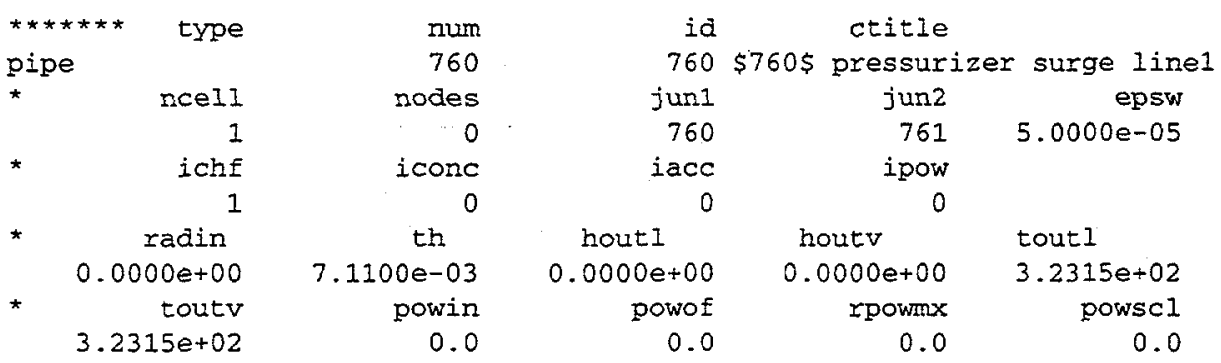

$\star$

$\star \mathrm{dx} * 1.0 \mathrm{e}$ 
* vol * 5.0874e-02e

* fa * r2 $5.0874 \mathrm{e}-02 \mathrm{e}$

* Eric * 1.0000 e-08

* elev * $2.032 e$

* hd * r2 2.5451e-01e

*nff *f le

*alp *f $0.0000 e+00 e$

* vl *f $0.0000 e+00 e$

* vr * E $0.0000 e+00 e$

$* \mathrm{tI} * \mathrm{f} 3.2315 \mathrm{e}+02 \mathrm{e}$

*tv *f $3.2315 \mathrm{e}+02 \mathrm{e}$

* p * E 7.32543e+05e

* pa $*$ f $0.0000 e+00 e$

$* * * \star * * *$ type num

pipe 761

* ncell nodes

2

ichf iconc

1

radin

$0.0000 e+00$

toutv

$7.1100 e-03$

th

powin

0.0

$3.2315 e+02$

* $\mathrm{dx} \quad * \mathrm{r} 21.762 \mathrm{e}$

* vol * r2 $8.9639 \mathrm{e}-02 \mathrm{e}$

* fa *r3 $5.0874 \mathrm{e}-02 \mathrm{e}$

* elev

* 0.175

0.175

$1.0000 e-08$

$0.175 e$

* ha

*alp * $\quad$ a $0.0000 e+00 e$

*v1 * $\quad$ v $0.0000 e+00 e$

$* v v *$ v $0.0000 e+00 e$

*tI * E $3.2315 e+02 e$

*tv * $\mathrm{t} \quad 3.2315 e+02 e$

$* \quad * \quad 7.23999 e+05$

* pa * E $0.0000 e+00 e$

$\star \star \star \star \star * * *$ tyape num

pipe 762

* ncell nodes

110

ichf

icone

radin

$0.0000 e+00$

toutv

$3.2315 e+02$

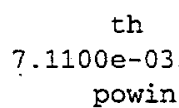

th

0

$7.06911 e+05 e$

hout I

$0.0000 e+00$
powof
0.0

id ctitle

$761 \$ 761 \$$ pressurizer surge line2

jun1 jun2

761

762

ipow

$5.0000 e-05$

epsw

houtv tout 1

$0.0000 e+00 \quad 3.2315 e+02$

rpowmx powscl

$\begin{array}{ll}0.0 & 0.0\end{array}$

$* d x \quad * 1.0 e$

* vol * $5.0874 e-02 e$

* fa * r2 5.0874e-02e

* fric * $0.175 \quad 1.0000 e-08$ e

* elev * $5.556 \mathrm{e}$

* hd *r2 2.5451e-01e

* nff * r2 le

*alp * f $0.0000 e+00 e$

$* \mathrm{vl} * \mathrm{f} 0.0000 \mathrm{e}+00 \mathrm{e}$

*vv *f $0.0000 e+00 e$

$\star t I * f \quad 3.2315 e+02 e$ 


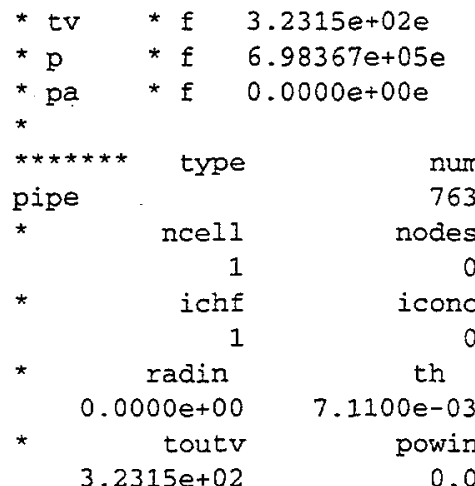

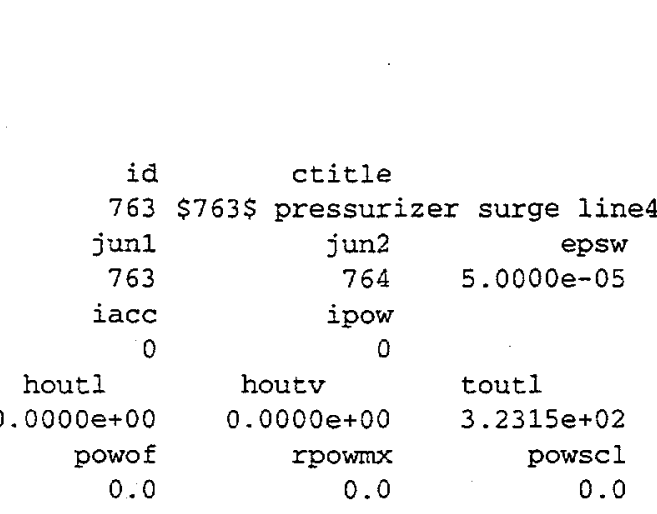

* dx * $\quad$ * 39810

* vol * 1.2200e-01e

* fa *r2 $5.0874 e-02 e$

* Eric * r2 $1.0000 e-08 e$

* elev * $5.556 \mathrm{e}$

* hd * r2 2.5451e-01e

* nff * r2 le

*alp *f $0.0000 e+00 e$

*VI *f $0.0000 e+00 e$

$* v v * f \quad 0.0000 e+00 e$

$* t 1 * \mathrm{f} 3.2315 \mathrm{e}+02 \mathrm{e}$

$* t v \quad * f \quad 3.2315 e+02 e$

$* \mathrm{p} * \mathrm{f} \quad .98367 \mathrm{e}+05 \mathrm{e}$

$* \mathrm{pa} *$ f $0.0000 e+00 e$

$* * * * * * *$ type

pipe type 764

pipe ncell nodes

* ichf 1 icon

* radin 1

$0.0000 e+00$

7. $1100 \mathrm{e}-03$

th

toutv

powin

0.0

id ctitle

$764 \$ 764 \$$ pressurizer surge lines

$3.1315 \mathrm{e}+02$

jun1

jun2

epsw

764

765

$5.0000 e-05$

iacc

houtl

$0.0000 e+00$

powof

0.0

ipow

houtv tout 1

$0.0000 e+00$

rpowinx

$3.1315 e+02$

powsc1

0.0

0.0

$* \mathrm{dx} * 1.0$

* vol * $5.0874 e-02 e$

* fa * r2 5.0874e-02e

* fric * $1.0000 e-08$

* elev * $5.556 \mathrm{e}$

* hd * r2 2.5451e-01e

* nff * r2 le

*alp *f $0.0000 e+00 e$

* v1 *f $0.0000 e+00 e$

* vv *f $0.0000 e+00 e$

$* t 1 * t 3.1315 e+02 e$

*tv *f $3.1315 e+02 e$

$* \mathrm{p} * \mathrm{f} 6.98367 \mathrm{e}+05 \mathrm{e}$

$* \mathrm{pa} *$ f $0.0000 e+00 e$

*

pipe 365

* ncell nodes

$0.0875 e$

13

0

ichf

icone

1

0

id ctitle

$765 \$ 765 \$$ pressurizer surge line6

junl

jun2

epsw

765

766

$5.0000 e-05$

iacc

ipow

0 
Page:

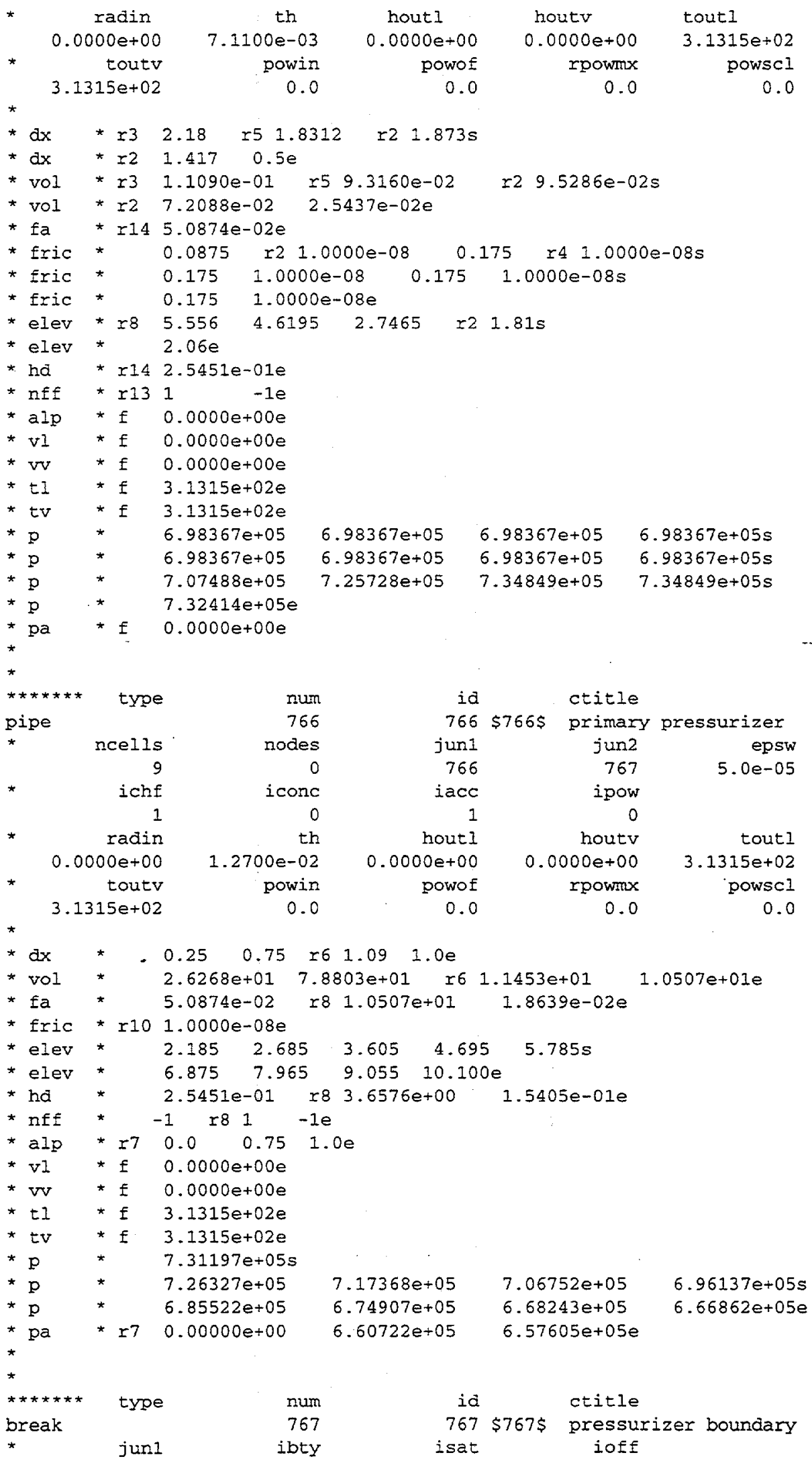




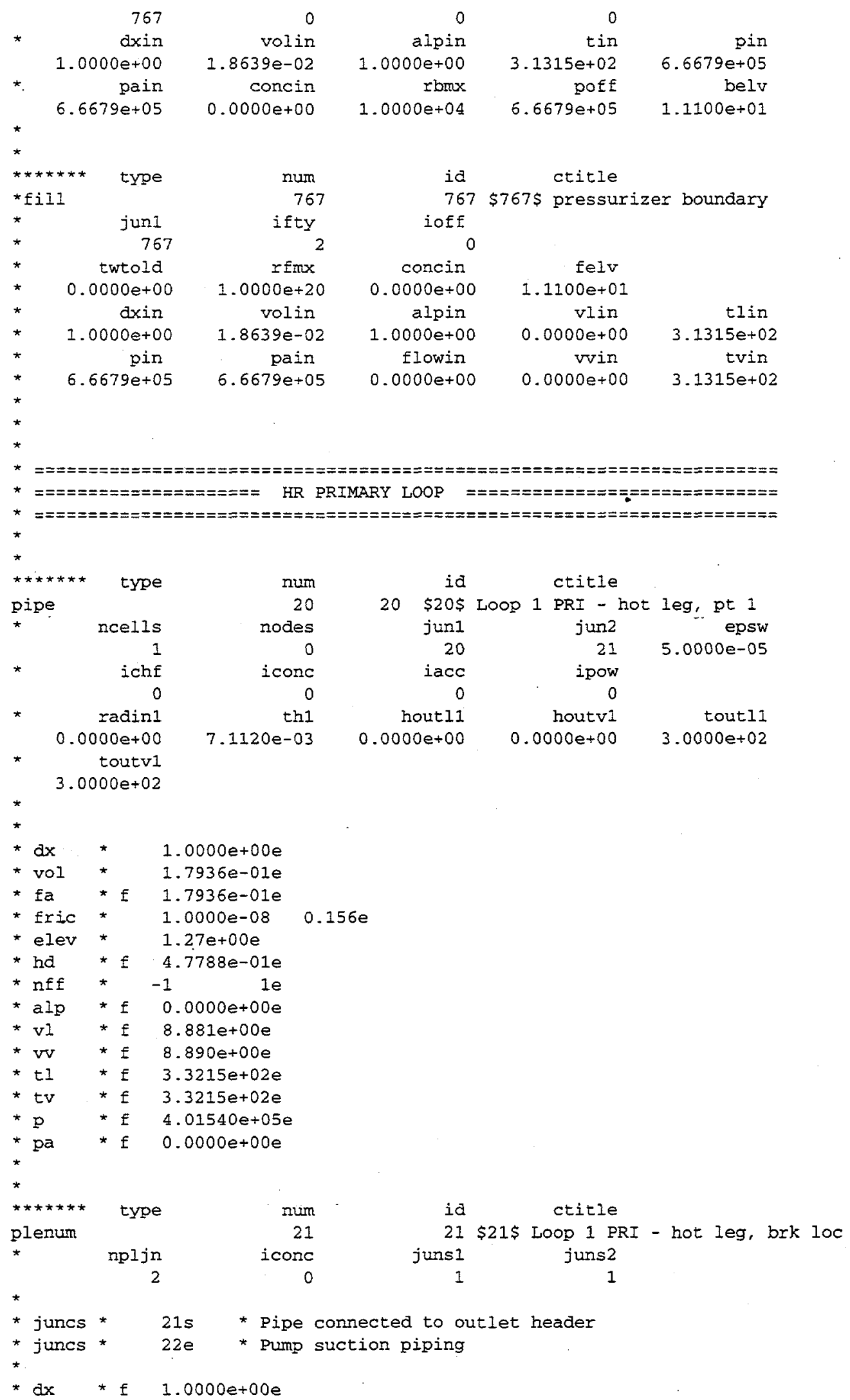




$\begin{array}{lll}\text { * vol * } & 1.7936 e-01 e \\ \text { * elev * } & 1.7700 e+00 e \\ \text { * alp * } & 0.0000 e+00 e \\ \text { *tl * } & 3.3215 e+02 e \\ \text { *tv * } & 3.3215 e+02 e \\ \text { * p * } & 3.89646 e+05 \\ \text { * pa * } & 0.0000 e+00 e\end{array}$

* pa $\star * 0.0000 e+00 e$

$\star * \star * \star * * *$ type num

pipe 2

* ncells nodes

* ichf 7 icone

* radin

$0.0000 e+00$

toutv

$3.0000 e+02$
* $r 2$

* $r 2$

* $\mathrm{E}$

$2.9469 e-01$

* vol

* $r$

$1.7936 \mathrm{e}-01 \mathrm{e}$

* Eric

* elev

* ha

* nff

* alp

* VI

* vv

$*$ t1

*tv

* $p$

$* p$

* pa

*

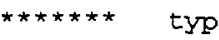

pipe

* ncells

1

ichf

radin 1

$0.0000 e+00$

toutv1

$3.0000 e+02$

$\star$

*

* dx *

* vol

* fa

* fric

* elev

* hd

* nff

* alp

* vl

* vv

* $t 1$

*tv

* $p$

$1.0000 e-08$

$3.0915 e+00$

$4.7788 \mathrm{e}-01 \mathrm{e}$

* $\mathrm{f}$

$0.0000 e+00 e$

$8.882 \mathrm{e}+00 \mathrm{e}$

* r7 $3.3215 e+02 e$

* r7 $3.3215 e+02 e$

$3.75557 e+05$

$3.40540 e+05$

$0.0000 e+00 e$
$7.11200-03$

$3.58040 e+05$

$3.42686 e+05$

$3.39467 e+05$

$3.38394 \mathrm{e}+05 \mathrm{e}$

title

$22 \$ 22 \$$ LOOp 1 PRI - hot leg, pt 2

juni

22

iacc

houtl

$0.0000 e \div 00$

0.0000

jun2

23

ipow

$5.0000 e-05$

epsw

houtv

tout I

$3.0000 e+02$

$r 51.0700 e+00 e$

r5 $1.9192 \mathrm{e}-01 \mathrm{e}$

0.156

r5 $1.0000 \mathrm{e}-08 \mathrm{e}$

$4.7345 e+00$ r5 $5.5560 e+00 e$

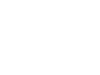

toutl1

houtv1

$0.0000 e+00$

$3.0000 e+02$ 
*pa *f $0.0000 e+00 e$

$*$

$\begin{array}{lrr}* * \star * * * * & \text { type } & \\ \text { pipe } & & \text { nu } \\ * & \text { ncells } & \text { nodes } \\ * & 13 & \\ & \text { ichf } & \text { icon } \\ * & 0 & \end{array}$

$0.0000 e+00 \quad 7.1120 e-03$

num

id ctitle

$24 \$ 24 \$$ Loop 1 PRI - hot leg, pt 3

0

un 1

24

jun2

epsw

iacc

25

$5.0000 e-05$

0

ipow

hout 1

houty

$0.0000 e+00$

$0.0000 e+00$

toutl

* toutv

$3.0000 e+02$

*

* r3 $1.0667 e+00$

$141.1500 e+00$

r6 $2.3750 e+00 e$

* vol

* $r 3$

$1.9132 \mathrm{e}-01$

$r 42.0626 \mathrm{e}-01$

r6

$4.2598 \mathrm{e}-01 \mathrm{e}$

fa

*

$1.7936 \mathrm{e}-01 \mathrm{e}$

* fric * $0.156 \quad$ r2 $1.0000 e-08$

* Eric * $0.156 \quad r 61.0000 e-08 e$

* elev

* hd

0.156

$r 3 \quad 1.0000 e-08 s$

*

$61.0000 e-08$

$2.8893 e+00 \quad r 10 \quad 2.3559 e+00 e$

* nff

* $\mathrm{f}$

$4.7788 e-01 e$

af $0.0000 e+00 e$

$* \mathrm{vI} * \mathrm{f} 8.882 \mathrm{e}+00 \mathrm{e}$

*VV *f $8.882 \mathrm{e}+00 \mathrm{e}$

$* t I * f \quad 3.3215 e+02 e$

$* t v * f 3.3215 e+02 e$

$* p \quad * 3.35408 \mathrm{e}+05$

$*$ * $\quad * 3.50702 e+05$

* $\quad * 3.38189 \mathrm{e}+05$

$* \mathrm{p} \quad * \quad 3.28664 \mathrm{e}+05 \mathrm{e}$

* pa *E $0.0000 e+00 e$

$3.44641 \mathrm{e}+05 \quad 3.53875 \mathrm{e}+05 \quad 3.51855 \mathrm{e}+05 \mathrm{~s}$

$3.49549 \mathrm{e}+05 \quad 3.48396 \mathrm{e}+05 \quad 3.40570 \mathrm{e}+05 \mathrm{~s}$

$3.35808 \mathrm{e}+05 \quad 3.33426 \mathrm{e}+05 \quad 3.31045 \mathrm{e}+05 \mathrm{~s}$

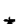

$* \star \star * * * * *$ type tyum

pipe

* ncells

1

* ich

nodes

num . is

id ctitle

\$25\$ Loop 1 PRI - hot leg bk loc

\section{0}

icone

radin.

$0.0000 e+00$

$7.1120 e-03$

toutv

$3.0000 e+02$

*

$* d x \quad * f \quad 1.0000 e+00 e$

* vol *f $1.7936 e-01 e$

* Ea * E $1.7936 \mathrm{e}-01 \mathrm{e}$

* fric * 1.0000 e-08

* elev * $2.3559 e+00 e$

* hd *f 4.7788e-01e

* nff * $f$ le

$*$ alp $* E 0.0000 e+00 e$

* vi * $\ldots 8.882 e+00 e$

*VV *E $8.882 \mathrm{e}+00 \mathrm{e}$

$* t I * f 3.3215 e+02 e$

*tv * $\pm 3.3215 e+02 e$

$* \mathrm{p} \quad * \mathrm{E} 3.26972 e+05 e$

* pa *E $0.0000 e+00 e$

$\star$

*

$\star * \star \star * * * *$ type num id ctitle 


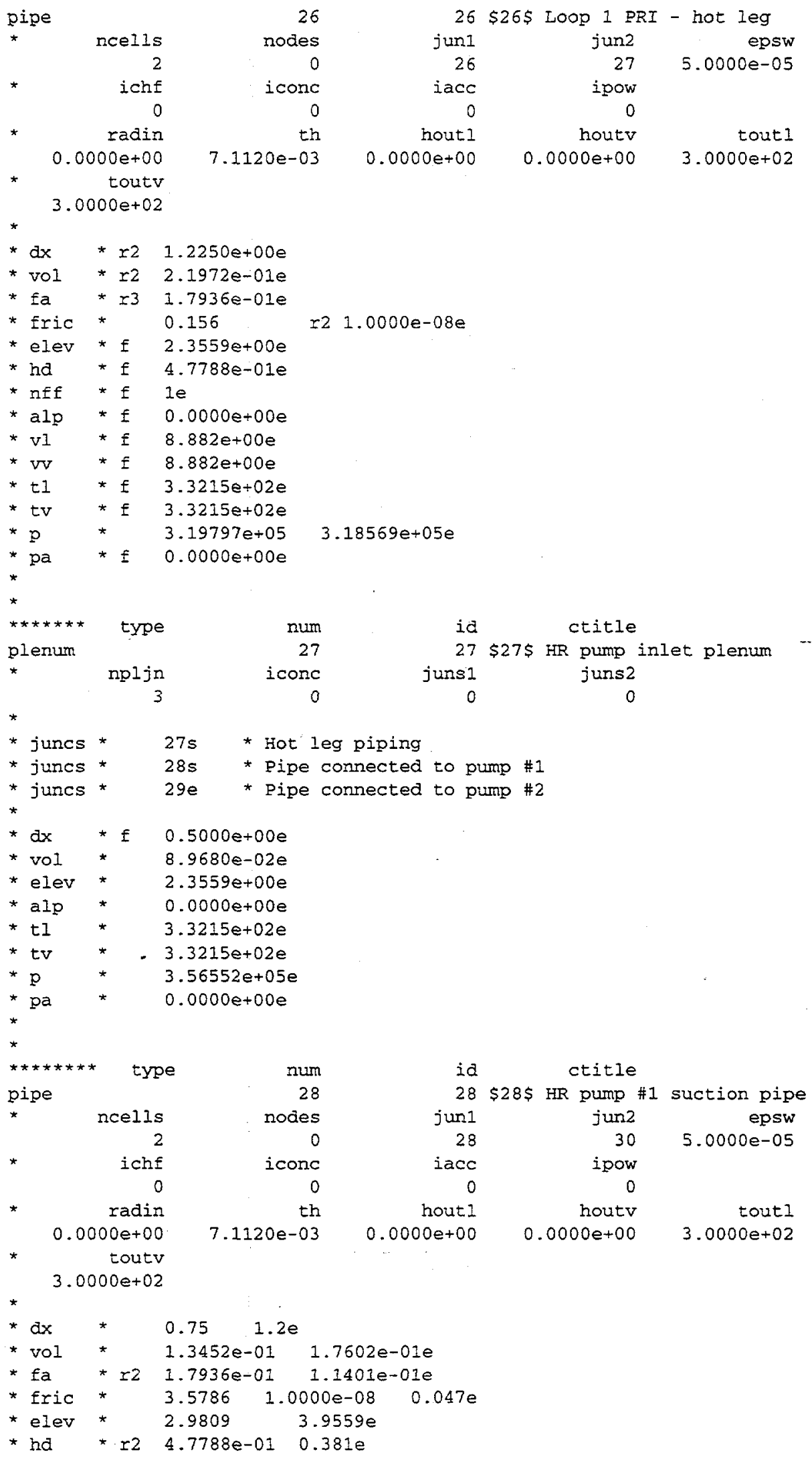

pipe

* ncells

nodes

$\star$

$0.00000+00$

ichf

radin

toutv

3. $0000 e+02$

* dx * r2 $1.2250 e+00 e$

* vol * r2 $2.1972 \mathrm{e}-01 \mathrm{e}$

* fa * r3 $1.7936 \mathrm{e}-01 \mathrm{e}$

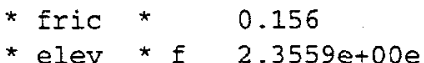

* hd *f $4.7788 \mathrm{e}-01 \mathrm{e}$

* nff * $f$ le

*alp *f $0.0000 e \div 00 e$

*Vl * E $8.882 \mathrm{e}+00 \mathrm{e}$

* vv * f $8.882 \mathrm{e}+00 \mathrm{e}$

*t1 * f $3.3215 e+02 e$

*tv * E $3.3215 e+02 e$

* $\mathrm{p} \quad$ * $3.19797 \mathrm{e}+05$

* pa *f $0.0000 e+00 e$

$r 21.0000 e-08 e$

*

$* * * * * * *$ type num id ctitle

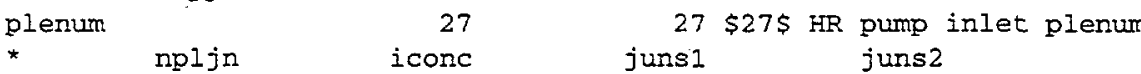

$\begin{array}{rrrr}* & 0 & 0 & 0\end{array}$

*

* juncs * $27 \mathrm{~s}$ * Hot leg piping

* juncs * $28 \mathrm{~s}$ * Pipe connected to pump \#1

* juncs * $29 \mathrm{e}$ * Pipe connected to pump \#2

* dx * $\quad$ e $0.5000 e+00 e$

* vol * $8.9680 e-02 e$

* elev * $2.3559 e+00 e$

*alp * $0.0000 e+00 e$

*tl * $3.3215 \mathrm{e}+02 \mathrm{e}$

* tv * $\quad$ - $3.3215 \mathrm{e}+02 \mathrm{e}$

* p * $\quad$ * $3.56552 \mathrm{e}+05 \mathrm{e}$

* pa * $0.0000 \mathrm{e}+00 \mathrm{e}$

*

*

$* \pi * * * * *$ type num id ctitie

pipe 28

* ncelis nodes

$28 \$ 28 \$$ HR pump \#1 suction pipe

2

ichf

0

radin

$0.0000 e+00$

$7.1120 e-03$

icone

th

toutv

3. $0000 e+02$

* $\mathrm{dx} \quad * \quad 0.75 \quad 1.2 \mathrm{e}$

* vol * $1.3452 \mathrm{e}-01 \quad 1.7602 \mathrm{e}-01 \mathrm{e}$

* fa * r2 1.7936e-01 1.1401e-01e

* Eric * $3.5786 \quad 1.0000 e-08 \quad 0.047 \mathrm{e}$

* elev * $2.9809 \quad 3.9559 \mathrm{e}$

* hd * r2 $4.7788 \mathrm{e}-01 \quad 0.381 \mathrm{e}$ 
$\begin{array}{lllll}* n f f & * & 1 & -1 & -1 e\end{array}$

*alp *f $0.0000 e+00 e$

* v1 * r2 $4.318 \mathrm{e}+00$

$6.793 e+00 e$

*vv * r2 $4.318 \mathrm{e}+00$

$6.793 e+00 e$

*t1 *f $3.3215 e+02 e$

$\star t v * f \quad 3.3215 \mathrm{e}+02 \mathrm{e}$

$* \mathrm{p} \quad * \quad 3.08331 e+05$

$* \mathrm{pa} \quad * \pm 0.0000 \mathrm{e}+00 \mathrm{e}$

$2.93292 e+05 e$

$* \star * \star * \star * *$ type num

pipe 29

* ncells nodes

* ichf iconc

* 0

$0.0000 e+00$

$7.1120 e-03$

toutv

$3.0000 e+02$

es

nc

0

hout 1

$0.0000 e+00$

$29 \$ 29 \$$ HR pump \#2 suction pipe jun1

29

iacc

0

$0.0000 e+00$

$3.0000 e \div 02$

$\star$

* dx * $0.5 \quad r 41.0 \quad r 21.1 \mathrm{e}$

* Vol * $8.968 \mathrm{e}-02$ 1.4669e-01 r3 $1.1401 \mathrm{e}-01$ r2 $1.2541 \mathrm{e}-01 \mathrm{e}$

* fa * r2 $1.7936 \mathrm{e}-01$ r6 1.1401e-01e

* fric * $0.0546 r 41.0000 e-08 \quad 0.159 \quad 1.0000 e-08 \quad 0.047 e$

* elev * r5 $2.3559 \quad 2.9059 \quad 4.0059 e$

* ha * r2 $4.7788 \mathrm{e}-01$ r6 $0.381 \mathrm{e}$

* riff * 1 r2 $-1 \quad$ r5 $1 e$

*alp * f $0.0000 e+00 e$

* v1 * r2 $4.564 e+00 \quad r 67.180 e+00 e$

*vv * r2 $4.564 e+00$ r6 $7.180 e+00 e$

*tl * E $3.3215 e+02 e$

*tv * $\mathrm{f} 3.3215 \mathrm{e}+02 \mathrm{e}$

* $\mathrm{p}$ * $3.45599 \mathrm{e}+05$

* $\mathrm{p} \quad$ * $3.24790 \mathrm{e}+05$

* pa * f 0.0000 e+00e

*

$\star$

$\star * \star * \star * *$ type nu

pump 30

* ncells nodes

* Thells 5

* ichf iconc

0

ipmptr
106

iqp 3 tr

radin

0.0000 e- 00

toutv

3. $3215 e+02$

tfro

40. $000 e+00$

terlo

$946.20 \mathrm{e}+00$

rhead

3. $9228 e+02$

omegan

6. $9639 e+01$

gp 3 in

0.0000 e +00

0
ipmpsv
0
igp $35 \mathrm{~s}$
0

1. 2700e-02

effmi

175.00

tfrl

$0.0000 e+00$

tEI 11

$0.0000 e+00$

rtork

8. $8578 e+02$

omgoff

$6.9639 e+01$

qp 3 off

$0.0000 e+00$ $\begin{array}{lll}3.39379 e+05 & 3.26515 e+05 & 3.25653 e+05 s \\ 3.14536 e+05 & 3.02963 e+05 e & \end{array}$

ctitie

$30 \$ 30 \$$ primary Loop \#1 pump

jun1 jun2 epsw

30

ipmpty

2

0

nqp 3 tb houtl

$0.0000 e+00$

$\operatorname{ten} 2$

$0.0000 e+00$

tfr 12

$0.0000 e+00$

rflow

$7.8863 e-01$

romgmx

1. $0000 e+20$

rop $3 \mathrm{mx}$

$0.0000 e+00$
$5.0000 e-05$

irp

npmpsv

0

houtv

$0.0000 e+00$

$3.32150+02$

tfr3

$0.0000 e+00$

tEx 13

$0.0000 e+00$

Irho

$9.8465 \mathrm{e}+02$

ongscl

$1.0000 e+00$

gp $3 \mathrm{scl}$

$0.0000 e+00$
1. $2600 e+00$

romega

$5.2360 e+01$

npmpsa
0 
* option

* dx * $0.5 \quad 1.1 e$

* vol * $\quad 5.7005 e-02$

* fa *f $1.1401 \mathrm{e}-01 \mathrm{e}$

* fric * $0.047 \quad 1.0000 e-08 \quad 0.206 e$

* elev * $4.8059 \mathrm{e}+00 \quad 5.0559 \mathrm{e}+00 \mathrm{e}$

* hd *f $3.8100 \mathrm{e}-01 \mathrm{e}$

$*$ nff * $\quad-1 \quad r 2$ le

$*$ alp *f $0.0000 e+00 e$

* vl *f $6.793 e+00 e$

*vv *f $6.793 e+00 e$

*tl *f $3.3215 \mathrm{e}+02 \mathrm{e}$

* tv * f $3.3215 \mathrm{e}+02 \mathrm{e}$

$* p \quad * 2.72618 \mathrm{e}+05$

* pa *f $0.0000 e+00 e$

* qppp *f $0.0000 e+00 e$

* matia * f $7 e$

* tw *f $3.7800 e+02 e$

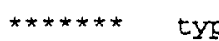

pump

* ncell

*

*

ipmptr

106

iqp 3 tr

radin

$0.0000 e-00$

toutv

$3.3215 e+02$

tfro

$40.000 e+00$

ter 10

$946.20 e+00$

rhead

$3.9228 e+02$

omegan

$6.9639 e+01$

cp3in

$0.0000 e+00$

option

2
$1.03566 e+05 e$

ctitle

1 \$31\$ primary Loop \#2 pump

jun1 jun2

33

irp

ipmpty

iconc

ipmpsv

iqp3sv

npmptb

nap 3 tib

nouti

$0.0000 e+00$

1.2700e-02

effmi

175.00

tfrl

$0.0000 e+00$

tfrl1

$0.0000 e+00$

rtork

$8.8578 e+02$

omgoff

$6.9639 e+01$

gp 3 off

$0.0000 e+00$

$\operatorname{tfr} 2$

$0.0000 e+00$

terl2

$0.0000 e+00$

rflow

$7.8863 e-01$

romgmx

$1.0000 e+20$

rap $3 \mathrm{mx}$

$0.0000 e+00$

$0.0000 e+00$

$0.0000 e+00$

rrho

$9.8465 e+02$

omgscl

$1.0000 e+00$

qp $3 \mathrm{scl}$

$0.0000 e+00$

\section{$5.0000 e-05$}

ipm

npmprf

nqp $3 r f$

tout

$3.3215 e+02$

tfrb

$1.2600 \mathrm{e}+00$

romega

$5.2360 e+01$

npmpsd

* dx * $0.5 \quad 1.1 \mathrm{e}$

* vol

* $\quad 5.7005 e-02$

$1.2541 e-01 e$

* fa

* $1.1401 \mathrm{e}-01 \mathrm{e}$

fric

*

$0.047 \quad 1.0000 e-08 \quad 0.206 e$

* elev

* ha

$\star \quad 4.8059 \mathrm{e}+00$

$5.0559 e+00 e$

* nff

$\star$ * $3.8100 e-01 e$

* alp

* r3 $1 e$

* vl * $7.180 \mathrm{e}+00 \mathrm{e}$

* $\pm \quad 0.0000 e+00 e$

$\star \mathrm{vv} * \pm 7.180 \mathrm{e}+00 \mathrm{e}$

* tI *E $3.3215 \mathrm{e}+02 \mathrm{e}$

*tv * $\quad$ tv $3.3215 e+02 \mathrm{e}$

* $\mathrm{p}$ * $2.93353 \mathrm{e}+05$

$1.04102 e+06 e$ 


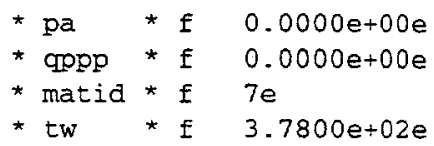

$* d x \quad * r 3 \quad 7.0830 e-01 e$

* vol * r3 $8.0753 e-02 e$

\section{$1.02907 e+06 \quad 1.02852 e+06 e$}

$0.0 \quad 0.0 \mathrm{~s}$

$1.0 \mathrm{e}+09 \quad 0.74 \mathrm{e}$

fmaxov

$1.0000 e+00$

houtv

$0.0000 e+00$

favive

$1.0000 e+00$

$0.0000 e+00$

xpos

$0.0000 e+00$ epsw

$5.0000 e-05$

nvtb2

$\begin{array}{rr}\text { nvrf } \\ 0 & 0 .\end{array}$ 
* fa * r4 1.1401e-01e

* fric * $0.206 \quad$ r2

* elev *f $5.0559 e+00 e$

* nd *f $3.8100 e-01 e$

* nef *f le

$*$ alp * $\quad 0.0000 e+00 e$

$\star v 1 * f 7.180 e+00 e$

$* \mathrm{vv} * \mathrm{f} 7.180 \mathrm{e}+00 \mathrm{e}$

* Il *f $3.3215 e+02 e$

* tv *f $3.3215 e+02 e$

$* \mathrm{p} * 1.03503 e+06$

$*$ pa $*$ E $0.0000 e+00 e$

*

$\begin{array}{ll}* \text { vtb1 * } & -1.0 e-09 \quad 0.0 e+00 \\ * \text { vtb1 * } & 50.0 \quad 0.36 \mathrm{~s}\end{array}$

* vtb1 * $100.0 \quad 0.74$

\section{$1.03378 e+06 \quad 1.03330 e+06 e$}
$\star * \star * * * *$ type num id ctitle

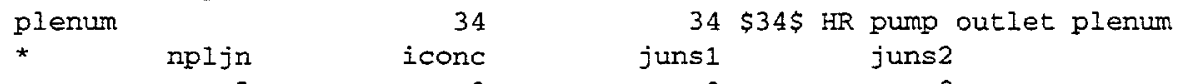

*

* juncs * $36 \mathrm{~s}$ * Pump discharge piping

* juncs * $34 \mathrm{~s}$ * Pipe connected to pump \#1

* juncs * $35 e$ * Pipe connected to pump \#2

$\star d x \quad * f \quad 0.5000 e+00 e$

* vol * $5.7005 e-02 e$

* elev * $5.0559 e+00 e$

*alp * $0.0000 \mathrm{e}+00 \mathrm{e}$

* t1 * $3.3215 \mathrm{e}+02 \mathrm{e}$

* tv * $3.3215 e+02 e$

* p * $\quad$ * $9.86905 e+05 e$

* pa * $\quad 0.0000 e+00 e$

*

$\star$

$\star * \star * \star * \star *$ type num

pipe 36

* ncells nodes

* iche $\quad$ iconc

* radin

$0.0000 e+00$

$7.1120 e-03$

toutv

$3.0000 e+02$

$\star$

* dx *f $1.3000 e+00 e$

$\star \operatorname{vol} * \mathrm{f} 1.4821 e-01 e$

* fa * f $1.1401 e-01 e$

* Eric * f $1.0000 e-08 \mathrm{e}$

* elev * f $5.0559 e+00 e$

* ha * f $3.8100 \mathrm{e}-01 \mathrm{e}$

* nff * f $1 e$

*alp * f $0.0000 e+00 e$

$\star v I * f \quad 1.397 e+01 e$

$\star v v * f \quad 1.397 e+01 e$

$\star t I \quad * f \quad 3.3215 e+02 e$

* tV * E $3.3215 \mathrm{e}+02 \mathrm{e}$

* $\mathrm{p} \quad$ * $\mathrm{f} 8.87878 \mathrm{e}+05 \mathrm{e}$

* pa *f $0.0000 \mathrm{e}+00 \mathrm{e}$ 


\section{*}

*

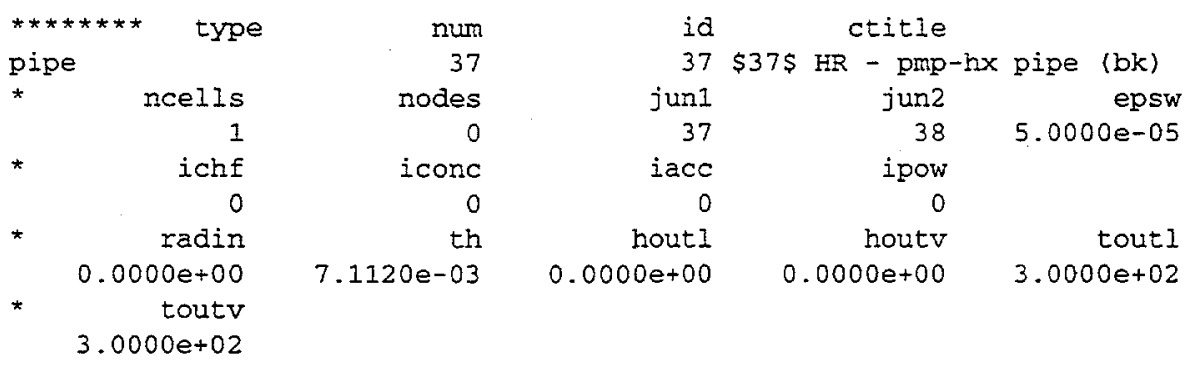

$\star$

* dx *f $1.0000 e+00 e$

* vol *f $1.1401 e-01 e$

* fa *f $1.1401 e-01 e$

* fric * f $1.0000 e-08 e$

* elev * f $5.0559 e+00 e$

* hd * f $3.8100 e-01 e$

*nff *f le

*alp * f $0.0000 e+00 e$

* vI * E $1.397 \mathrm{e}+01 \mathrm{e}$

*v *f $1.397 e+01 e$

*tI *f $3.3215 e+02 e$

$\star \mathrm{tv} \quad \star f \quad 3.3215 \mathrm{e}+02 \mathrm{e}$

* $\mathrm{p}$ * $\mathrm{f} 8.84160 \mathrm{e}+05 \mathrm{e}$

* pa * E $0.0000 e+00 e$

*

$\star$

$* * * * * * * *$ type nu

pipe 38

* ncelis nodes

* $\begin{array}{rr}1 & 0 \\ \text { *hf } & \text { iconc }\end{array}$

* radir 0

$0.0000 e+00 \quad 7.1120 e-03$

* toutv

$3.0000 e+02$

$\star$

* dx. * f $1.3000 e+00 e$

* vol * f $1.4821 e-01 e$

* fa * f $1.1401 e-01 e$

* fric * f $1.0000 e-08 \mathrm{e}$

* elev * f $5.0559 e+00 e$

* hd *f $3.8100 \mathrm{e}-01 \mathrm{e}$

* nff * $\mathrm{f} 1 \mathrm{e}$

*alp * f $0.0000 e+00 e$

*vl *f $1.397 e+01 e$

* vv *f $1.397 e+01 e$

*tl *f $3.3215 \mathrm{e}+02 \mathrm{e}$

*tv * $\mathrm{f} \quad 3.3215 \mathrm{e}+02 \mathrm{e}$

*p * $\mathrm{p} 8.80443 \mathrm{e}+05 \mathrm{e}$

* pa *

$\star$

$\star * \star * * * *$ type num

plenum 40

$\begin{array}{rr}\text { * } \operatorname{spljn} & \text { iconc } \\ 3 & 0\end{array}$

$40 \$ 40 \$ \mathrm{HR} h \mathrm{hx}$ inlet plenum juns1 juns2

* juncs * $39 s$ * Pump aischarge piping 
* juncs * 48s * Pipe connected to $h x \# 1$

* juncs * $49 \mathrm{e}$ * Pipe connected to $\mathrm{hx}$ \#2

*

*dx *f $0.5000 e+00 e$

* vol * 5.7005e-02e

* elev * 5.0559e+00e

*alp * $0.0000 e+00 e$

$* t 1 * 3.3215 e+02 e$

* tv * $\quad$ * $3.3215 e+02 e$

$* p \quad * \quad 9.73667 e+05 e$

* pa * $\quad 0.0000 e+00 e$

*

$\star * * * * * *$ type num

pipe 48

* ncelis nodes

* ichf $\quad$ iconc

* radin th

$0.0000 e+00$

$7.1120 e-03$

toutv

$3.0000 e+02$

*

* dx

* vol

2.125

r2 $0.5 e$

* r3 $1.1401 \mathrm{e}-01$

$5.7005 e-02$

$1.6175 \mathrm{e}$

*a faic *

3.8574

$5.6558 \mathrm{e}-01 \mathrm{e}$

* elev * \& $5.0559 \mathrm{e}+00 \mathrm{e}$

* ho * $=3 \quad 3.8100 e-01$

* nff * 221 r2 $-1 e$

*aIp *f $0.0000 e+00 e$

* v1 * r3 $7.237 \mathrm{e}+00 \quad 1.458 \mathrm{e}+00 \mathrm{e}$

* vv * r3. $7.237 \mathrm{e}+00 \quad 1.458 \mathrm{e}+00 \mathrm{e}$

$* t 1 * \mathrm{f} 3.3215 e+02 e$

*tv *f $3.3215 e+02 e$

$* \mathrm{p} \quad * 8.47673 \mathrm{e}+05$

* pa *f $0.0000 e+00 e$

$8.41956 e+05 \quad 8.43273 e+05 e$

*

*

$* * * * * * * *$ type num

pipe 50

* ncells nodes

* $\begin{array}{rr}4 & 0 \\ \text { ichE } & \text { iconc }\end{array}$

(

radin

$0.0000 e+00$

$7.11200-03$

toutv

$3.0000 e+02$

$* d x \quad * \pm 9.9000 e-01 e$

* vol *f 5.5992e-01e

* fa * $\quad$ f $5.6558 \mathrm{e}-01 \mathrm{e}$

* Eric * $1.0000 e-08 e$

* elev * $\neq 5.0559 e+00 e$

* ha *f $1.0210 e-02 e$

* nff * $\quad-1$ r3 1 - $1 e$

*alp *f $0.0000 e+00 e$

* Vl * $\mathrm{f} 1.458 \mathrm{e}+00 \mathrm{e}$

* vV *E $1.458 \mathrm{e}+00 \mathrm{e}$

$\star t 1 \quad * f \quad 3.3215 e+02 e$ 
* tv *f $3.3215 e+02 e$

* p * 8.39292e+05

$* \mathrm{pa} * \mathrm{f} 0.0000 \mathrm{e}+00 \mathrm{e}$
$8.36841 e+05$
$8.34381 e+05$
$8.31913 e+05 e$

*

$\star$

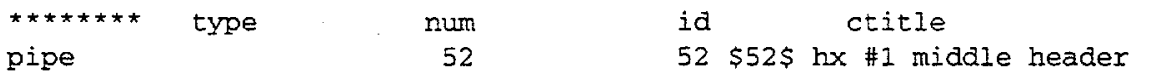

pipe

ncells

3

ins

un1

jun2

epsw

* ichs

radin

iconc

52

54

$5.0000 e-05$

$0.0000 e+00$

toutv

$3.0000 e+02$

$7.1120 e^{\text {th }}$

iacc

0

ipow

houtl

houty

tout I

$0.0000 e+00$

$0.0000 e+00$

3. $0000 e+02$

$* d x \quad$ * $\quad 5.0000 \mathrm{e}-01$

$1.5000 e+00$

$5.0000 e-01 e$

* vol * $\quad 1.6175 e+00$

$4.8525 e+00$

1. $6175 \mathrm{e}+00 \mathrm{e}$

* fa *

$5.6558 e-01$

* fric * f $1.0000 e-08 \mathrm{e}$

* elev * $5.0559 e+00$

r2 $3.2350 e+00$

$5.6558 e-01 e$

* ha

$3.5559 e+00 \mathrm{e}$

* nff

*alp * a $0.0000 e+00 e$

$1.0210 e-02 r 22.0295 e+00$

1. $0210 \mathrm{e}-02 \mathrm{e}$

* VI * $1.458 e+00$

* vv * $1.458 e+00$

*tI *f $3.3215 \mathrm{e}+02 \mathrm{e}$

*tv *E $3.3215 \mathrm{e}+02 \mathrm{e}$

* $\mathrm{p}$ * $8.30356 \mathrm{e}+05$

* pa * $\quad 0.0000 e+00 e$

*

$\star$

$* \star \star \star * \star * *$ type nu

pipe 54

r2 $2.544 e-01 \quad 1.455 e+00 e$

r2 $2.544 \mathrm{e}-01 \quad 1.455 \mathrm{e}+00 \mathrm{e}$

$\star$

ncells

nodes

$8.37618 e+05$

$8.44881 e+05 e$

*

ichf

radin

$0.0000 e+00$

toutv

$3.0000 e+02$

*

* dx * f $9.9000 e-01 \mathrm{e}$

$\star$ vol * f $5.5992 e-01 \mathrm{e}$

* fa * f $5.6558 \mathrm{e}-01 \mathrm{e}$

* fric * f $1.0000 e-08 \mathrm{e}$

* elev *f $3.5559 e+00 e$

* ha *f $1.0210 \mathrm{e}-02 \mathrm{e}$

* nff * -1 $\quad$ r3 1 - le

*alp * f $0.0000 \mathrm{e}+00 \mathrm{e}$

* vl * f $1.455 e+00 e$

* vv *f $1.455 \mathrm{e}+00 \mathrm{e}$

*tl * $\mathrm{t} 3.3215 \mathrm{e}+02 \mathrm{e}$

*tv ty $\mathrm{f} 3.3215 \mathrm{e}+02 \mathrm{e}$

* $\mathrm{p} \quad$ * $8.40895 \mathrm{e}+05$

* pa $\quad$ f $0.0000 e+00 e$

$8.38411 e+05 \quad 8.35920 e+05 \quad 8.33423 e+05 e$

*

$\star$

$\star * * * * * * *$ type num

pipe

ncells

56

nodes

$56 \$ 56 \$ \mathrm{HR} h \times \# 1$ outlet pipe

junl jun2 epsw 


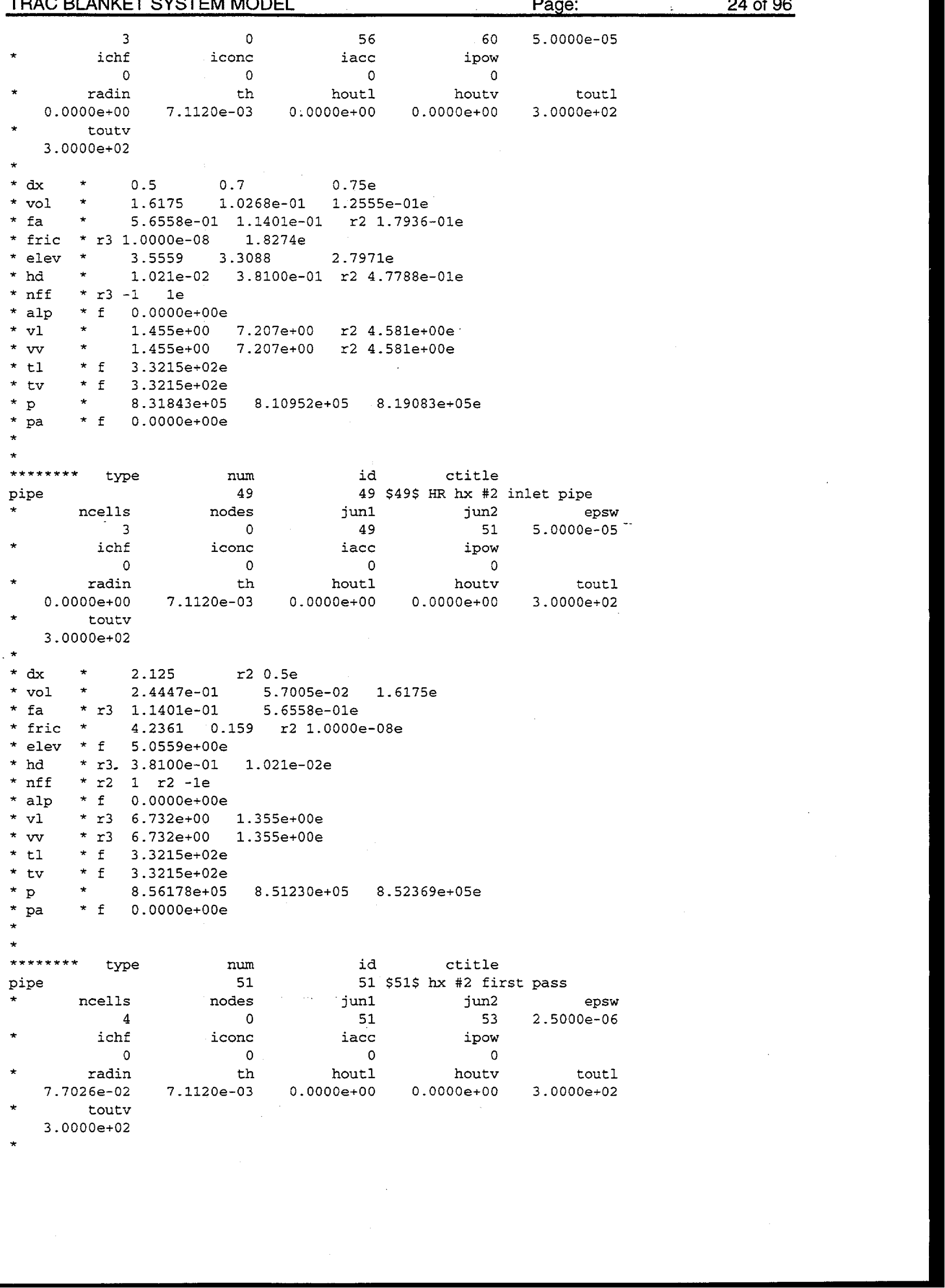


* $d x \quad * E \quad 9.9000 e-01 e$

* vol *f 5.5992e-01e

* fa *f $5.6558 \mathrm{e}-01 \mathrm{e}$

* fric * f $1.0000 e-08 e$

* elev * f $5.0559 e+00 e$

* ho * $\mathrm{f} 1.0210 \mathrm{e}-02 \mathrm{e}$

* nfE * -1 r3 1 - 1 e

*alp * $* 0.0000 \mathrm{e}+00 \mathrm{e}$

*V1 * $\mathrm{fl} 1.355 \mathrm{e}+00 \mathrm{e}$

* vv * f $1.355 e+00 \mathrm{e}$

*t1*f $3.3215 \mathrm{e}+02 \mathrm{e}$

$\star t v \star E .3 .3215 e+02 e$

* $\mathrm{p} \quad$ * $8.48910 \mathrm{e}+05$

* pa $*$ f $0.0000 e+00 e$
$8.46754 e+05$
$8.44590 e+05$
$8.42419 e+05 e$

*

$\star$

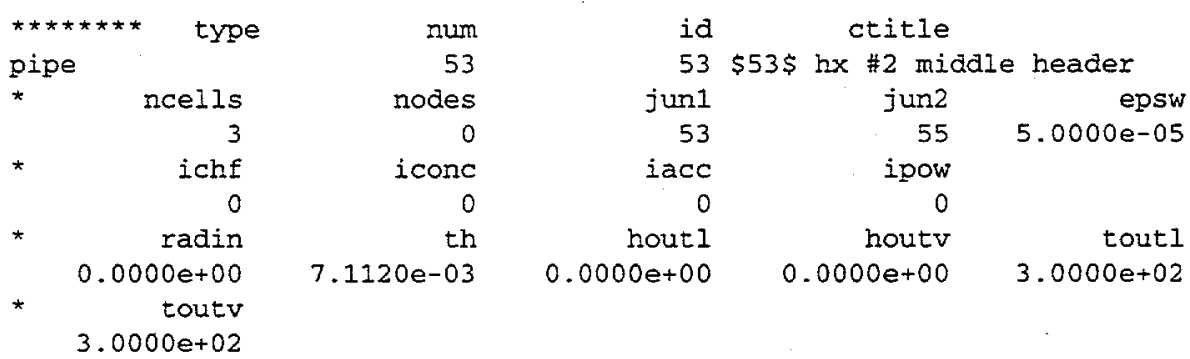

*

* dx $\quad$ * $\quad 5.0000 \mathrm{e}-01$

* vol * $\quad 1.6175 \mathrm{e}+00$

$1.5000 \mathrm{e}+00$

5. $0000 e-01 e$

* Ea

$5.6558 e-01$

* Eric

* f $1.0000 e-08$ e

* elev * $5.0559 \mathrm{e}+00$

* ha

* nff

* alp

* $\quad \begin{array}{lr}5.0559 e+00 & 4.3059 e+00 \\ * \quad 1.0210 e-02 \text { r2 } 2.0295 e+00\end{array}$

$4.8525 e+00$

$1.6175 \mathrm{e}+00 \mathrm{e}$

$\Upsilon 23.2350 e+00$

$5.6558 e-01 e$

* -1 r2 $1-1 e$

* f $0.0000 e+00 e$

*vI * $\quad$ * $1.355 e+00$

$\star v \mathrm{v} *$

* $t$ * $\mathrm{f} 3.3215 \mathrm{e}+02 \mathrm{e}$

* tV *f $3.3215 e+02 e$

* $\mathrm{p} \quad$ \& $8.41044 \mathrm{e}+05$

* pa. *f $0.0000 e+00 e$

r2 $2.367 e-01 \quad 1.353 e+00 e$

r2 $2.367 e-01 \quad 1.353 e+00 e$

$\star$

$* * * * * * * *$ type num

pipe ncells nodes

$8.48308 e+05 \quad 8.55572 e+05 e$

$$
\text { ichf }
$$

0

0

radin

$7.7026 e-02$

toutv

3. 0000 e+02

*

* dx *

* vol *f $5.5992 \mathrm{e}-01 \mathrm{e}$

* fa *

* Eric *f $1.0000 \mathrm{e}-08 \mathrm{e}$

* elev * f $3.5559 \mathrm{e}+00 \mathrm{e}$

* ha *f $1.0210 e-02 e$

* nff * $\quad 1 \quad r 31 \quad-1 e$

*alp * E $0.0000 e+00 e$ 


\begin{tabular}{|c|c|c|}
\hline v1 & $E$ & $1.353 e+00 e$ \\
\hline vv & $* \pm$ & $1.353 e+00 e$ \\
\hline & * $f$ & $3.3215 e+0$ \\
\hline & * 1 & $3.3215 e$ \\
\hline & * & $8.52107 e+c$ \\
\hline & & 0.0000 et \\
\hline
\end{tabular}
$8.49921 e+05$
$8.47729 e+05$
$8.45530 \mathrm{e}+05 \mathrm{e}$

*

$$
\begin{aligned}
& * \\
& * \star \star \star \star \star \star * *
\end{aligned}
$$$$
* \star * \star * \star * * \text { type }
$$

th

*

nodes
0
iconc
0
th
$7.1120 \mathrm{e}-03$
iacc


Report:

Section:

Date:

Page:

houtl1

$7.1120 e-03$

$0.0000 e+00$

houtv1

$0.0000 e+00$

tout11

$0.0000 e+00$

toutvi

$3.0000 e+02$

*

* dx * $\quad$ * $1.7500 \mathrm{e}+00 \mathrm{e}$

* vol * $3.1388 \mathrm{e}-01 \mathrm{e}$

* fa *f $1.7936 \mathrm{e}-01 \mathrm{e}$

* fric * $1.0000 e-08$

* elev * $2.3559 e+00 e$

* ha * f 4.7788e-01e

* nff * f $1 e$

*alp *f $0.0000 e+00 e$

* vI *f $8.843 e+00 e$

*vv *f $8.843 e+00 e$

$\star t I * f \quad 3.3215 e+02 e$

*tv * f $3.3215 \mathrm{e}+02 \mathrm{e}$

* p * f $7.76337 e+05 e$

* pa *f $0.0000 e+00 e$

$* * * * * * *$ type num

pipe

* ncells

ncells nodes

* ichf

radin 1

$0.0000 e+00$

toutv1

$3.0000 e+02$

*

$* d x \quad * \quad 1.0000 e+00 e$

* vol * $1.7936 \mathrm{e}-01 \mathrm{e}$

* fa $* \mathrm{f} 1.7936 \mathrm{e}-01 \mathrm{e}$

* fric * 0.156

* elev * $2.3559 e+00 e$

* hd * $\mathrm{f} 4.7788 \mathrm{e}-01 \mathrm{e}$

* nff *f le

*alp * E $0.0000 e+00 e$

* v1 * f $8.843 e+00 e$

* VV *f $8.843 e+00 e$

$* \mathrm{tI} * \mathrm{f} \quad 3.3215 \mathrm{e}+02 \mathrm{e}$

*tv *f $3.3215 e+02 e$

*p *f $7.68926 e+05 e$

*pa *f $0.0000 e+00 e$

$* * * * * * * *$ type num

pipe 64

* ncells nodes

* $13 \quad$.

ichf icone

radin th

$0.0000 e+00$

$7.1120 e-03$

toutv

$3.0000 e+02$ id ctitle

$63 \$ 63 \$ \mathrm{HR}$ Cold leg pipe (bk) jun1

63

iacc

0

houtI1

$0.0000 e+00$

$000 e+00$ jun2

$5.0000 e-05$

ipow

0

tout 11

$3.0000 e+02$
$0.156 \mathrm{e}$

$1.0000 e-08 e$

ctitle

$64 \$ 64 \$$ HR Cold leg pipe

jun1 jun2 epsw

$64 \quad 65 \quad 5.0000 e-05$

iacc

ipow

houtl houtv tout I

$0.0000 e+00 \quad 0.0000 e+00 \quad 3.0000 e+02$

$* d x \quad * r 6 \quad 1.7583 e+00 \quad r 41.0125 e+00 \quad r 31.0667 e+00 e$ 


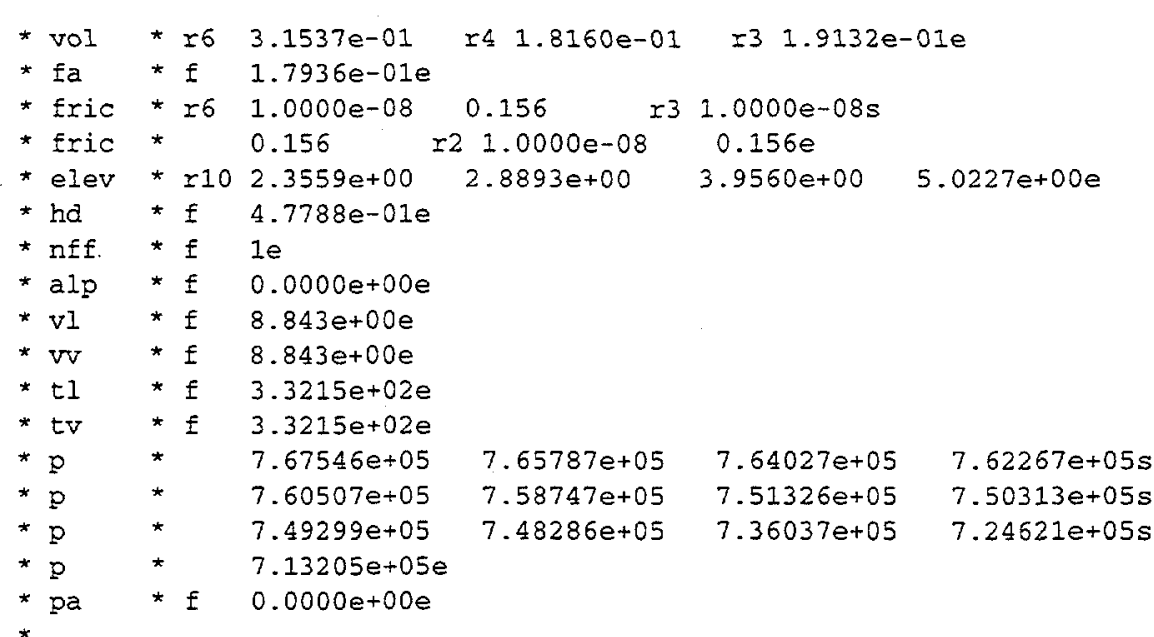

*

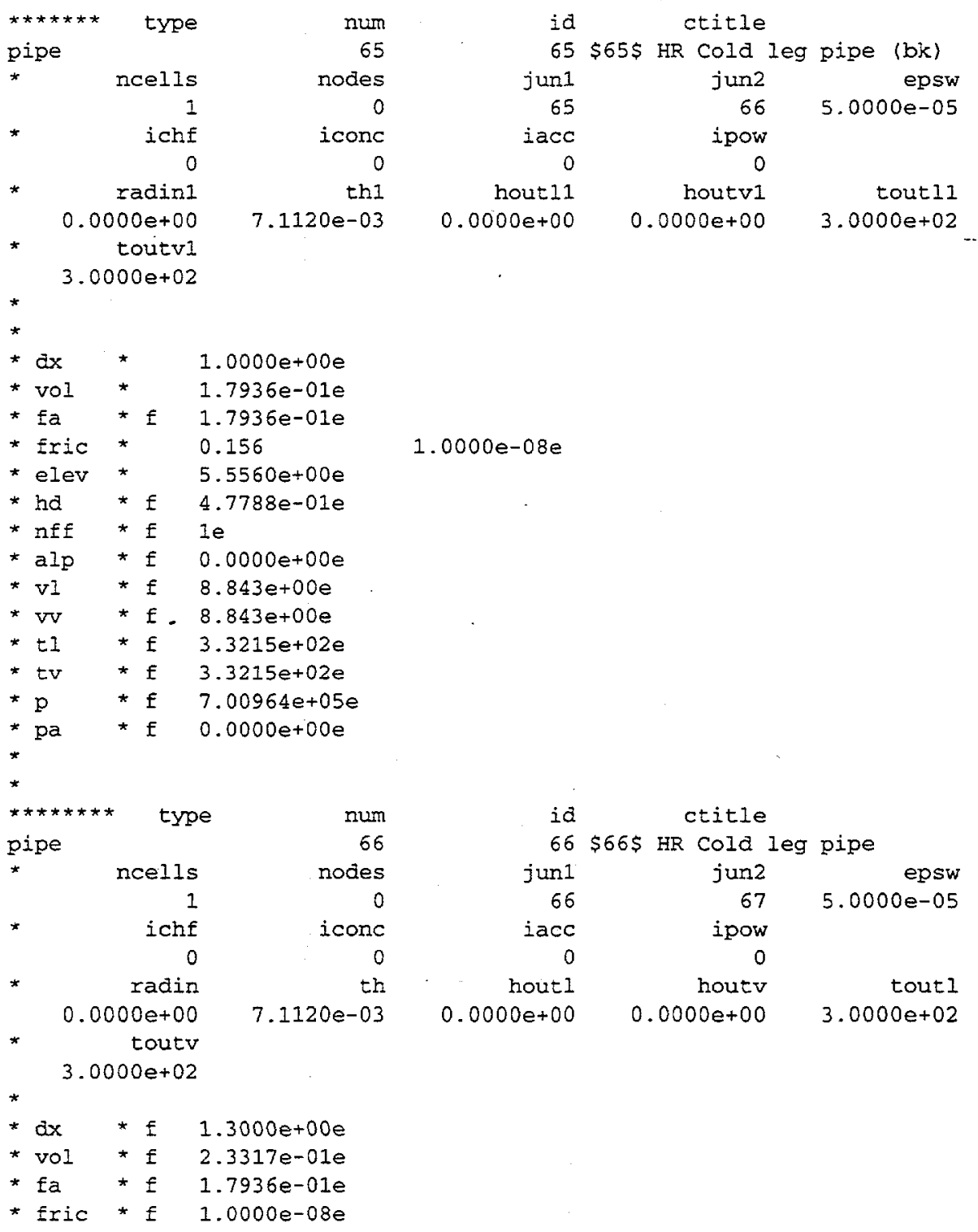




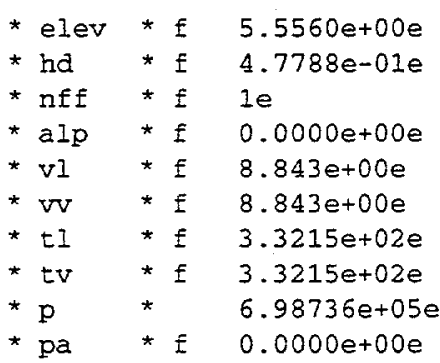

$\star$

$* * * * * * *$ type num

plenum 67

npljn iconc

$3 \quad 0$

* juncs * $67 \mathrm{~s}$

* juncs * $854 \mathrm{~s}$

* Cold leg piping

* Piping between HR COld Leg plenums

* juncs * $851 e$

* Valve connected to Cavity vessel (FR side)

$\begin{array}{llll}\text { dx } \quad \text { *2 } & 0.5 & 0.25 e\end{array}$

* vol * $\quad$ * $8.9680 \mathrm{e}-02 \mathrm{e}$

* elev * $5.5560 e+00 e$

*alp * $\quad$ * $0.0000 \mathrm{e}+00 \mathrm{e}$

* $t 1 * 3.2315 \mathrm{e}+02 \mathrm{e}$

*tv * $3.2315 \mathrm{e}+02 \mathrm{e}$

* p * $\mathrm{p} .97585 \mathrm{e}+05 \mathrm{e}$

* pa * $0.0000 e+00 e$

*

$\star$

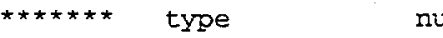

valve $\quad 854$

* ncells nodes

* ichf 2 iconc

* ivtr 0 ivs

109

ivtrov

$r \sin x$

$1.0000 e+01$

radin

$0.0000 e-00$

toutv

$3.3300 \mathrm{e}+00$

*

*

*dx *f $0.5 e$

* vol *f $8.9680 \mathrm{e}-02 \mathrm{e}$

* fa * f $1.7936 \mathrm{e}-01 \mathrm{e}$

* fric * f $1.0000 e-08 e$

* elev * $\neq 5.5560 \mathrm{e}$

* hd *f $4.7788 e-01 e$

* nff * $f$ 1e

*alp * e $0.0 e$

*VI * $\mathrm{VI} 8.843 e+00 \mathrm{e}$

* vv $\star \mathrm{f} 8.843 e+00 \mathrm{e}$

*tl * $t$. $3.2300 e+02 e$

*tv *f $3.2300 e+02 e$

*p *f $6.97585 e+05 e$ id ctitle

$67 \$ 67 \$$ HR break (HR side)

juns1 juns2

$$
11
$$




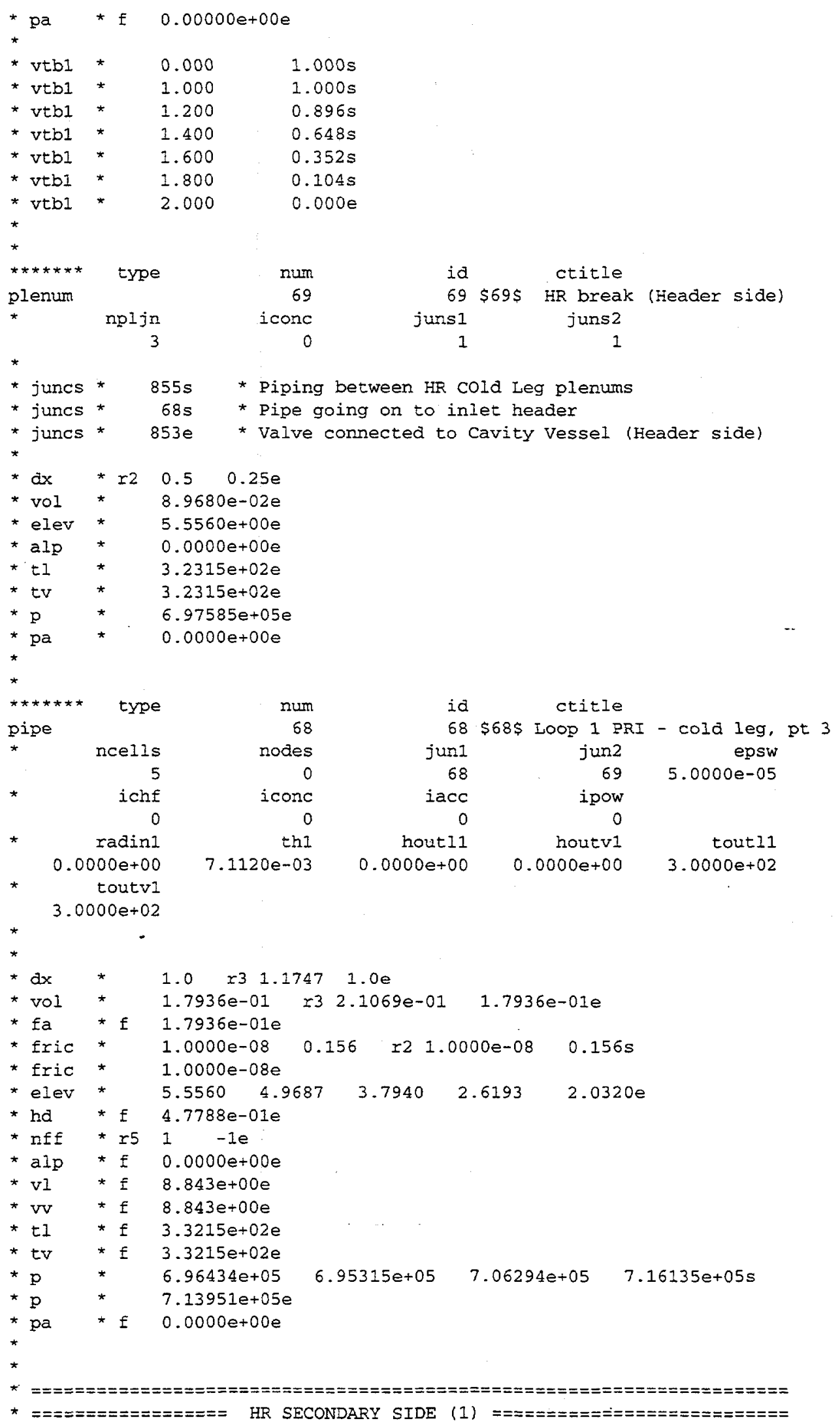




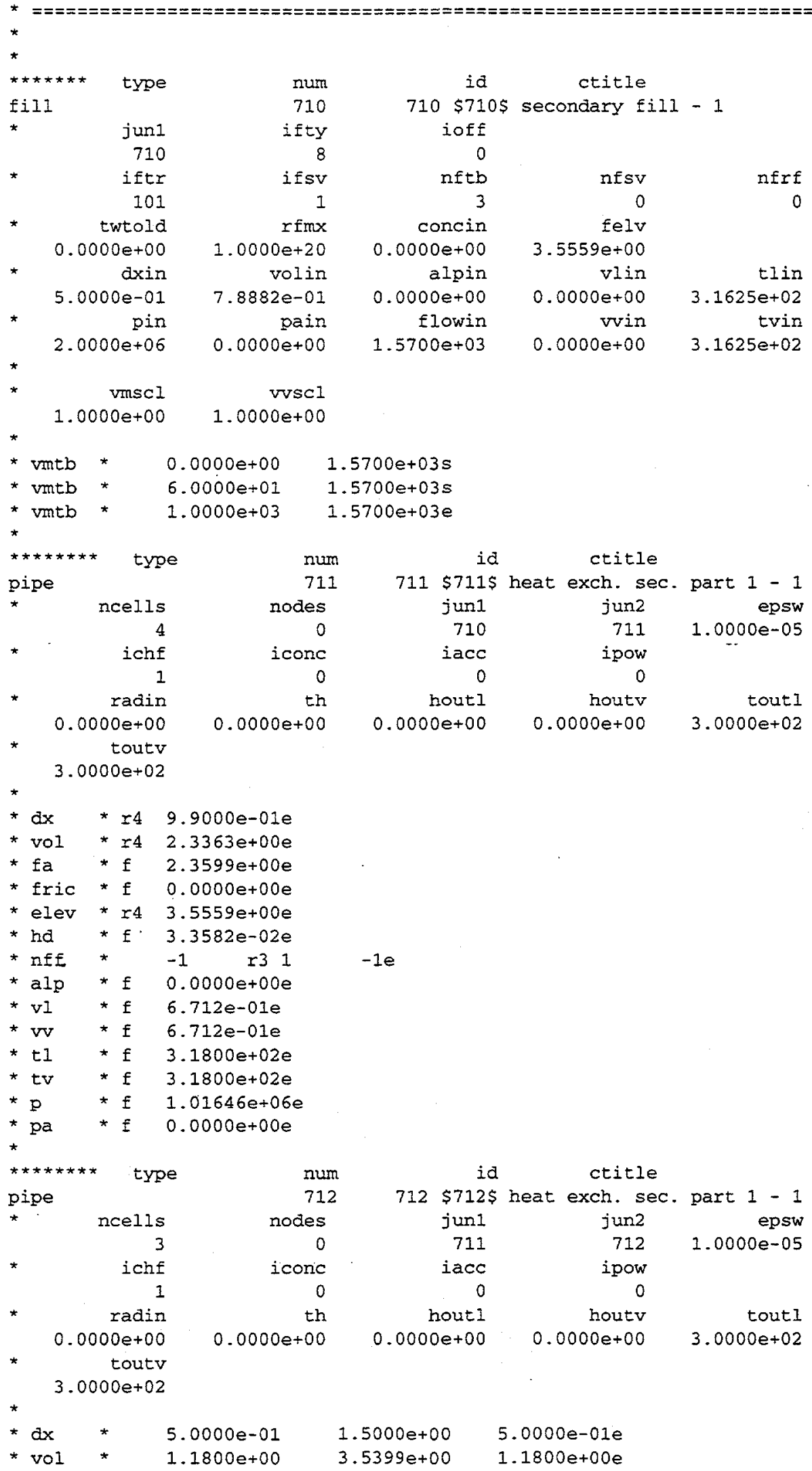


*fa *f $2.3599 e+00 e$

* fric * f $1.0000 e-08 e$

* elev * $3.5559 e+00$

* hd *f $3.3582 e-02 e$

* nff * $\quad-1 \quad r 21-1 e$

$* \operatorname{alp} * f \quad 0.0000 e+00 e$

$* \mathrm{vI} * \mathrm{f} \quad 6.712 \mathrm{e}-01 \mathrm{e}$

*vv *f $6.712 \mathrm{e}-01 \mathrm{e}$

$* t 1 *$ f $3.1800 e+02 e$

$*$ tV * f $3.1800 e+02 e$

$* \mathrm{p} * 1.01589 e+06$

*pa *f $0.0000 e+00 e$
$4.3059 e+00$
$5.0559 e+00 e$

$* \star * * * * * *$

type

$1.00845 e+06 \quad 1.00100 e+06 e$

pipe

* ncells

*

4
* $\quad r$ fh
1

nodes

id

ctitle

icone

radin

toutv
$+\quad 3.0000 e+02$

$0.0000 e+00$

$713 \$ 713 \$$ heat exch. sec jun 1 jun.2

- 1

712

713

1.0000

iacc

ipow

0

houty

ee-05

*

*dx * r4 $9.9000 e-01 e$

* vol * r4 $2.3363 \mathrm{e}+00 \mathrm{e}$

$\star \mathrm{fa}$ * $\mathrm{f} 2.3599 \mathrm{e}+00 \mathrm{e}$

* Eric * $f \quad 1.0000 e-08 e$

* elev * $E 5.0559 e+00 e$

* hd *f $3.3582 e-02 e$

* nff * $\quad-1 \quad r 31 \quad-1 e$

*alp *f $0.0000 e+00 e$

*v1 *f $6.712 \mathrm{e}-01 \mathrm{e}$

* vv *f $6.712 \mathrm{e}-01 \mathrm{e}$

*tI * E $3.1800 e+02 e$

*tv * f $3.1800 e+02 e$

$* p \quad * f \quad 1.00073 e+06 e$

*pa *f $0.0000 e+00 e$

*

$\star * * * * * *$ type num

break 714

* junl ibty

713

dxin

volin

5. 0000 e-01

pain

$7.8882 e-01$

concin

$0.0000 e+00$

$0.0000 e+00$

tout 1

$.0000 e+02$

$0.0000 e+00$

$0.0000 e+00$

$$
\begin{array}{r}
\text { id } \\
\text { 714 } \$ 714 \$ \\
\text { isat } \\
0 \\
\text { alpin }
\end{array}
$$

$0.0000 e+00$

rbmx

1. $0000 e+20$ ctitle

secondary break - 1

ioff

$$
\operatorname{tin}
$$

3. $1800 e+02$

poff

$0.0000 e+00$

$1.0000 e+06$

5.055

t

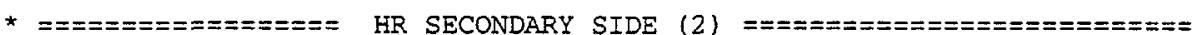

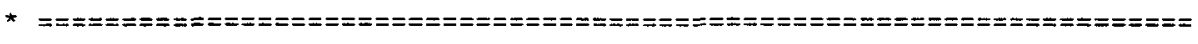

$\star$

$\star$

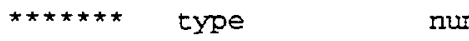

fill 730

* jun1 ifty

730

iftr

101

twtold

$0.0000 e+00$

ifty

ifsv

1

$\operatorname{Imx}$

$1.0000 e+20$

$0.0000 e+00$

id

ctitle

$730 \$ 730 \$$ secondary fill - 2

ioff

$n=t b$

Etb

concin

$n \leq s+$

nExf

$3.5559 e+00$ 
Report:

Section:

Date:

Page:

$\begin{array}{rr}\text { vin } & t \text { tin } \\ 0.0000 e+00 & 3.1625 e+02 \\ \text { vvin } & t v i n \\ 0.0000 e+00 & 3.1625 e+02\end{array}$

alpin

$0.0000 e+00$

flowin

$5.0000 e-01$

7.8882e-01

pain

$2.0000 e+06$

$0.0000 e+00$

$1.5700 \mathrm{e}+03$

vvscl

$1.0000 e+00$

$1.0000 e+00$

*

* vmtb

$0.0000 e+00$

$1.5700 \mathrm{e}+03 \mathrm{~s}$

* vmtb

$6.0000 e+01$

$1.5700 \mathrm{e}+03 \mathrm{~s}$

$* \star * * * * * *$ type num

pipe

$1.0000 e+03$

$1.5700 e+03 e$ ncelis 731

* $\quad 4$

* $\quad \begin{array}{r}\operatorname{ich} f \\ 1\end{array}$

* radin

$0.0000 e+00$

toutv

$3.0000 e+02$

*

*dx *r4 $9.9000 e-01 e$

* vol * r4 $2.3363 \mathrm{e}+00 \mathrm{e}$

* fa *f $2.3599 e+00 e$

* fric *f $0.0000 e+00 e$

* elev * r4 $3.5559 e+00 \mathrm{e}$

* hd * f $3.3582 \mathrm{e}-02 \mathrm{e}$

* nff * $\quad-1 \quad r 31$

*alp *f $0.0000 e+00 e$

$* \mathrm{v} 1 * \mathrm{f} 6.711 \mathrm{e}-01 \mathrm{e}$

$*$ vv *E $6.711 e-01 e$

$* t 1 * f 3.1800 e+02 e$

$* t v * f 3.1800 e+02 e$

$* \mathrm{p} \quad * \mathrm{f} 1.01631 \mathrm{e}+06 \mathrm{e}$

* pa *f $0.0000 e+00 e$

$\star$

$\star \star \star \star * \star * \star *$ type num

pipe 732

* ncells nodes

* ichf iconc

1

radin

$0.0000 e+00$

toutv

$3.0000 e+02$

*

* dx * $\quad$ * $5.0000 e-01$

* vol * $1.1800 e+00$

*fa *f $2.3599 e+00 e$

* fric * f $1.0000 e-08 e$

* elev * $3.5559 e+00$

* hd *f $3.3582 \mathrm{e}-02 \mathrm{e}$

* nff * $\quad-1 \quad r 21 \quad-1 e$

*alp *f $0.0000 e+00 e$

$* \mathrm{vl} * \mathrm{f} \quad 6.711 \mathrm{e}-01 \mathrm{e}$

*v *f 6.711e-01e

$*$ t1 *f $3.1800 e+02 e$

$*$ tv $\quad$ f $3.1800 e+02 e$

$* \mathrm{p} * 1.01589 \mathrm{e}+06$
$1.5000 e+00$
$5.0000 \mathrm{e}-01 \mathrm{e}$
$3.5399 e+00$
1. $1800 \mathrm{e}+00 \mathrm{e}$
$4.3059 e+00$
$5.0559 e+00 e$

houtv
$0.0000 e+00$

toutl

h. sec. part $1-2$

jun2

731

epsw

pow

hout I

$3.0000 e+02$

2 
* pa $\quad$ f $0.0000 e+00 e$

*

$\star * * * * \star * *$ type num

pipe 733

* ncelis nodes

* $\begin{array}{rr}4 & 0 \\ \text { *chf } & \text { iconc } \\ 1 & 0\end{array}$

* radin

$0.0000 e+00$

$0.0000 e+00$

toutv

$3.0000 e+02$

$\star$

* dx * r4 $9.9000 e-01 e$

* vol * r4 $2.3363 e+00 e$

* fa * f $2.3599 e+00 e$

* fric * f $1.0000 e-08 e$

* elev * f $5.0559 e+00 e$

* ha *f $3.3582 e-02 e$

$*$ nff * $\quad-1 \quad$ r3 1 - $1 e$

*alp * f $0.0000 e+00 e$

* v1 *f $6.711 \mathrm{e}-01 \mathrm{e}$

* vv *f $6.711 \mathrm{e}-01 \mathrm{e}$

$* \mathrm{tI} * \pm 3.1800 \mathrm{e}+02 \mathrm{e}$

*tv *f $3.1800 e+02 e$

* $p \quad * f \quad 1.00074 e+06 e$

* pa *f $0.0000 e+00 e$ $\star * * \pi * \star *$ type num num break 734

* jun1 ibty

* axir volin

5.0000e-01 $7.8882 e-01$

concin

* paín

$0.0000 e+00$

$0.0000 e+00$

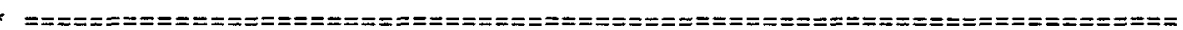

*

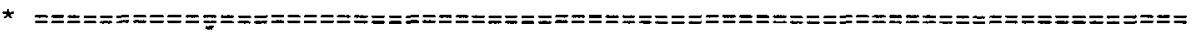

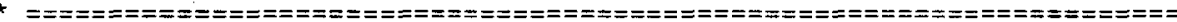

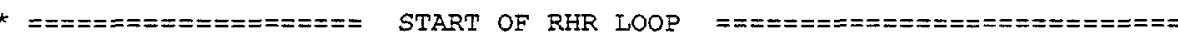

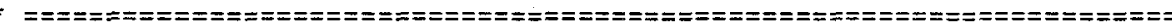

$* * * * * * *$ type

* ncells

621

nodes

* ich $f$

* radin1

7. $7026 e-02$

toutv1

$3.0000 e+02$

*

* $\mathrm{dx}$ * $1.0000 \mathrm{e}+00 \mathrm{e}$

* vol * $\quad 1.8639 e-02 e$

* fa * $\mathrm{f} \quad 1.8639 \mathrm{e}-02 \mathrm{e}$ 


\begin{tabular}{|c|c|c|}
\hline * fric & * & $1.0000 e-08$ \\
\hline elev & * & $1.2700 \mathrm{e}+00 \mathrm{e}$ \\
\hline ha & * $\mathrm{f}$ & $1.5405 e-01 e$ \\
\hline * nff & * & -1 \\
\hline * alp & * $\mathrm{f}$ & $0.0000 \mathrm{e}+00 \mathrm{e}$ \\
\hline * $v 1$ & $* f$ & $0.0000 e+00 e$ \\
\hline$\star v \mathrm{v}$ & * $\mathrm{f}$ & $0.0000 e+00 e$ \\
\hline * $t I$ & $* f$ & $3.3215 e+02 e$ \\
\hline$\star t v$ & $* E$ & $3.3215 \mathrm{e}+02 \mathrm{e}$ \\
\hline * $p$ & $* E$ & $4.53240 e+05$ \\
\hline * pa & $* f$ & $0.0000 e+00$ \\
\hline
\end{tabular}

$\star * * * * * * *$ type num

pipe 623

* ncells nodes

$\begin{array}{rr}10 & \text { iconc }\end{array}$

* ichf icon

* radin

$7.7026 \mathrm{e}-02$

toutv

$3.0000 e+02$

$7.1120 e-03$
$0.25 e$

$0.0000 e+00$

$0.0000 e+00$

$3.0000 e+02$

R - hot leg, pt 2

jun1 jun2 epsw

$623 \quad 624 \quad 5.0000 e-05$

ipow

0

tout

*

$* \mathrm{dx} \quad *$ r4 $1.0715 \mathrm{e}+00 \mathrm{r} 6 \quad 1.1000 \mathrm{e}+00 \mathrm{e}$

* vol * r4 $1.9972 \mathrm{e}-02 r 62.0503 \mathrm{e}-02 \mathrm{e}$

* fa *f $1.8639 \mathrm{e}-02 \mathrm{e}$

* fric * 0.25 r3 $1.0 e-08 \quad 0.25$ r2 $1.0 e-08 s$

* fric * 0.0425 r3 $1.0 e-08 \mathrm{e}$

* elev * $\quad 1.8058 e+00 \quad 2.8773 e+00 \quad 3.9488 e+00 \quad 5.0203 e+00 r 6 \quad 5.5560 e+00 e$

* ha *f $1.5405 \mathrm{e}-01 \mathrm{e}$

*nff *f le

$*$ alp * $\neq 0.0000 e+00 e$

$*$ vI *E $0.0000 e+00 e$

*Vv * f $0.0000 e+00 e$

$* t 1 \quad * r 103.3215 e+02 e$

* tv * r10 $3.3215 \mathrm{e}+02 \mathrm{e}$

* $\mathrm{p}$ * $4.48068 \mathrm{e}+05$

* p * r6 4.11865e+05e

*pa *f $0.0000 e+00 e$

pipe type

$4.37724 e+05 \quad 4.27380 e+05 \quad 4.17036 e+05 s$

ncells

624

1

nodes

1 che
0

radin1

$0.0000 e+00$

toutv1

$3.0000 e+02$

$7.1120 e^{\operatorname{th} 1}-03$

icone

0 
*t1 *f $3.1315 e+02 e$

$\star t v * f 3.1315 e+02 e$

* $\mathrm{p} \quad * \mathrm{f} 4.11865 e+05 e$

* pa *f $0.0000 e+00 e$

*

$\star * * * * * * \star$ type num

pipe 625

* ncells nodes

* $\begin{array}{rr}18 & 0 \\ 0 & i \operatorname{con} f\end{array}$

* radin

$0.0000 e+00$

toutv

$3.0000 e+02$

Page:

*

* dx *r3 $1.2000 e+00 r 7 \quad 1.9143 e+00 r 7 \quad 2.0286 e+00 \quad 1.0000 e+00 e$

* vol * r3 2.2367e-02r7 3.5680e-02r7 3.7810e-02 1.8639e-02e

* fa *f $1.8639 e-02 e$

* fric * $0.25 \quad 0.251 \quad 1.0 e-08 \quad 0.25$ r6 $1.0 e-085$

* fric * 0.25 r6 $1.0 \mathrm{e}-080.25 \quad 0.25 \mathrm{e}$

* elev * 4.9560e+00 3.7560e+00 2.5560e+00r15 1.9560e+00e

* ha * $\quad$ t $1.5405 e-01 e$

*nff *f le

*alp * f $0.0000 e+00 e$

*VI * E $0.0000 e+00 e$

* vv * f $0.0000 e+00 e$

$* t I * f \quad 3.1315 e+02 e$

*tv *f $3.1315 e+02 e$

*p * $4.17704 \mathrm{e}+05$

* pa * f $0.0000 e+00 e$

pump

* ncells

* $\quad$ ichf

* ipmptr

105

* iqp3zr

* radín

$0.0000 e+00$

toutv

$3.0000 e+02$

$t \pm x 0$

$473.10 e+00$

$\operatorname{ter} 10$

$946.20 e+00$

rhead

1. $4553 \mathrm{e}+02$

omegan

$0.0000 e+02$

qp 3 in

$0.0000 e+00$

option

* dx *f $1.0000 e-00 e$

* vol *f $1.8639 \mathrm{e}-02 \mathrm{e}$

* fa *f $1.8639 e-02 e$

7.1120
$4.29389 e+05 \quad 4.41074 e+05$ I15 $4.46917 e+05 e$

num

630

nodes

iconc

ipmpsv

imp $35 v$

effri

175.00

$\operatorname{ter} 1$

$0.0000 e+00$

tEII

$0.0000 e+00$

rtork

$6.0177 e+00$

ongoff

$0.9435 e \div 02$

qp3off

0.0000 et 00

$0.0000 e+00$

tex 12

$0.0000 e+00$

rflow

$7.9838 e-02$

romgmx

$1.0000 e+20$

rap $3 \mathrm{mx}$

$0.0000 e+00$ id ctitle

30 \$630\$ RIR - pump

in1 jun2

640

irp

npmpsv

nop $35 \mathrm{sv}$

hout

$0.0000 e+00$

$\operatorname{ten} 3$

$0.0000 e+00$

tEr 13

$0.0000 e+00$

rrho

$0.9850 e+03$

omgscI

$1.0000 \mathrm{e}+00$

ap $3 \mathrm{scl}$

$0.0000 e+00$
3. $0000 e+02$

5. eposw

ipm

npmprf

nqp $3 r f$

tout 1

teris

1. $2600 e+00$

romega

1. $0472 e+02$

npmpsd 


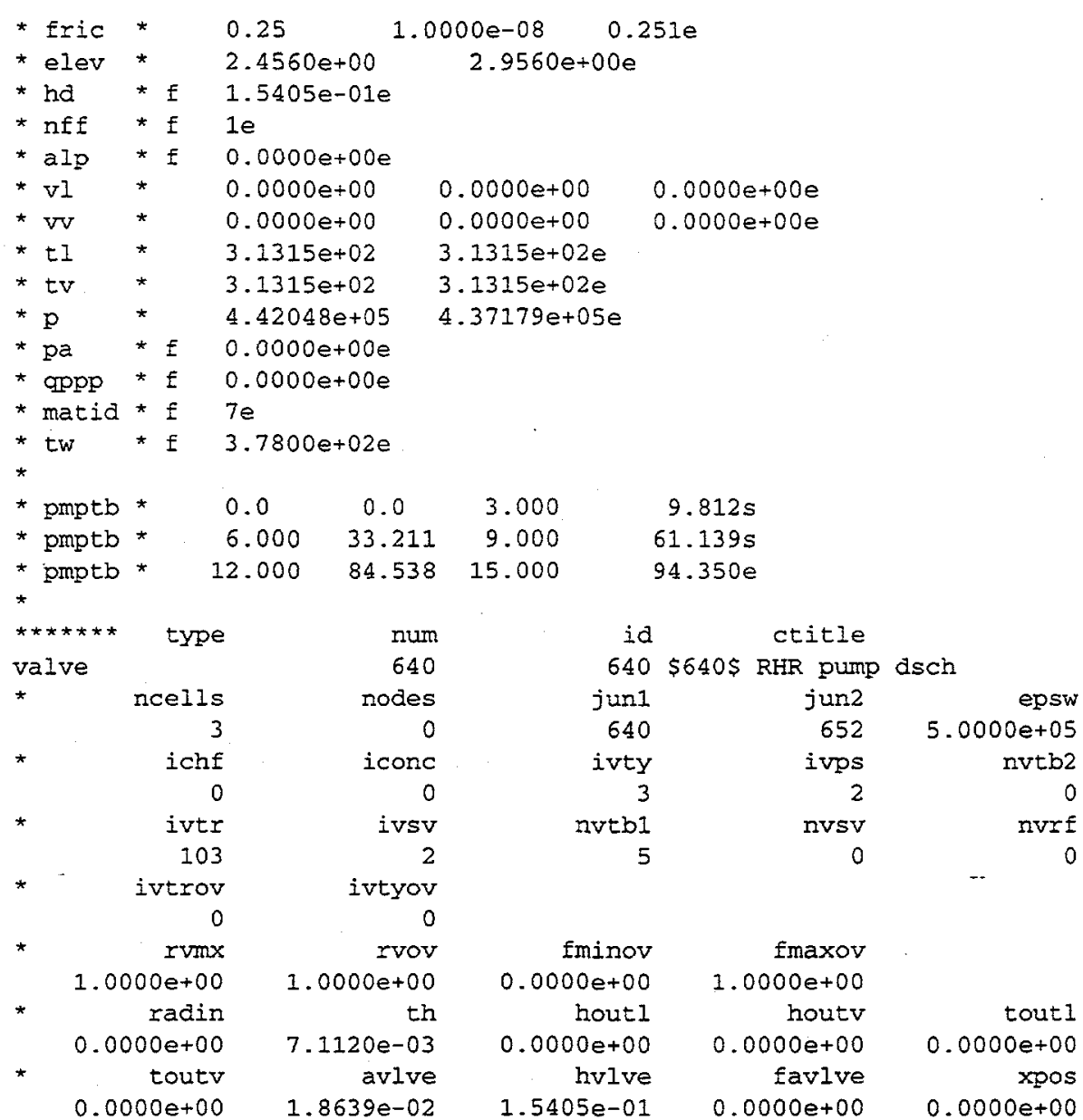

$\star$

$\star d x \quad * \quad 1.0000 e+00$

* vol * $1.8639 \mathrm{e}-02$
0.797
$0.203 e$

* fa * r3 $1.8639 e-02$

r3 $1.8639 e-02$

$2.2679 e-02 e$

* Eric

* E $2.9560 \mathrm{e}+00 \mathrm{e}$

* r03 $1.5405 \mathrm{e}-01 \quad 1.0210 \mathrm{e}-02 \mathrm{e}$

* hd * rff $\quad$ ro3 $1.5405 e-01$ - 1 - 1.0

*alp * *a $0.0000 e+00 e$

$* \mathrm{vl} * \mathrm{f} \quad 0.0000 \mathrm{e}+00 \mathrm{e}$

*vV *f $0.0000 e+00 e$

$* t I * f \quad 3.1315 e+02 e$

*tv *f $3.1315 \mathrm{e}+02 \mathrm{e}$

* p *f 4.37179e+05

* pa *f $0.0000 e+00 e$

$\star$

* vtb1 * $\quad-1.0 e-09 \quad 0.0 e+00$

* vtb1 * $50.00 .36 \mathrm{~s}$

* vtb1 * 100.00 .712

$r 27.23900 e+05 e$

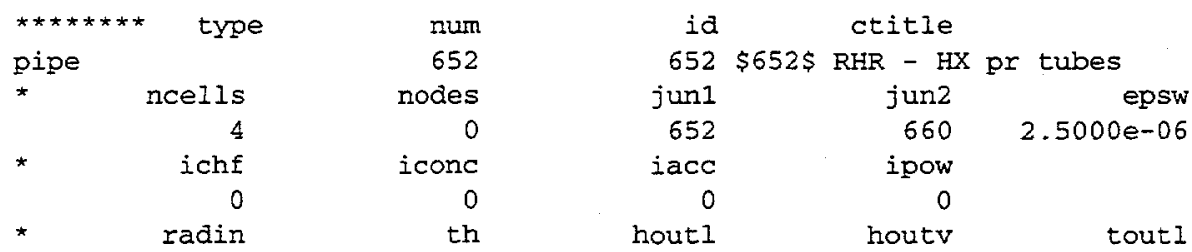


$3.0000 e+02$

*

* dx *f $9.9000 e-01 e$

* vol * f $2.2452 \mathrm{e}-02 \mathrm{e}$

$* \mathrm{fa} * \mathrm{E} 2.2679 \mathrm{e}-02 \mathrm{e}$

* Eric * $1.0000 \mathrm{e}-08 \mathrm{e}$

* elev * $f \quad 2.9560 e+00 e$

* hd * $1.0210 \mathrm{e}-02 \mathrm{e}$

* nff * $\quad-1$ r3 1 -1e

*alp * E $0.0000 e+00 e$

$*$ v1 *E $0.0000 \mathrm{e}+00 \mathrm{e}$

*vv * * $0.0000 \mathrm{e}+00 \mathrm{e}$

$* t 1 \quad * \pm 3.1315 \mathrm{e}+02 \mathrm{e}$

*tv * $3.1315 e+02 e$

$* \mathrm{p} \quad * \pm 7.23900 \mathrm{e}+05 \mathrm{e}$

* pa * $0.0000 \mathrm{e}+00 \mathrm{e}$

*

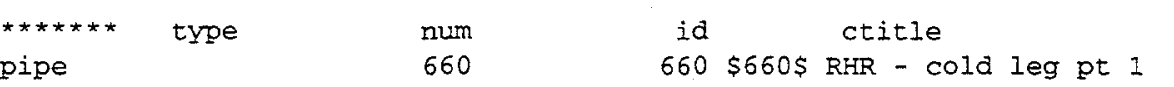
pipe cold leg from heat exchanger exit ncells nodes

jun1 $\begin{array}{rr}16 & 0 \\ \text { ichf } & \text { iconc } \\ 0 & 0\end{array}$

toutv
$3.0000 \mathrm{e}+02$

$7.1120 e-03$

660
iacc

0.0000 hout1

jun2

661

ipow

*

* $d x \quad * \quad 0.203 \quad 0.797 \simeq 6 \quad 1.0677 e+00 r 6 \quad 2.0667 e+00 r 2 \quad 1.3000 e+00 e$

* vol * $2.6281 \mathrm{e}-02 \quad 1.4855 \mathrm{e}-02$ I6 $1.9900 \mathrm{e}-02$ r6 $3.8520 \mathrm{e}-02 \mathrm{~s}$

* vol * r2 $2.4230 \mathrm{e}-02 \mathrm{e}$

* fa * 2.2579e-02 r16 $1.8639 \mathrm{e}-02 \mathrm{e}$

* Eric * r4 $1.0000 e-08 \quad 0.25$ r3 $1.0000 e-08 \quad 0.251 \quad$ r5 $1.0000 e-08 s$

* Eric *

* elev

* hd

* nff

*alp

* vi

$*$ vv

$* t$

$*$ tv

$*$ Ev

$* \mathrm{p} * \quad$ 7.23900e+05

* p * 7.23900e+05

$* \mathrm{p} \quad * \quad 7.23900 \mathrm{e}+05$

* $\mathrm{p}$ * $7.23900 e+05$

* pa * e $0.0000 e+00 e$

*

$\star \star \star \star \star \star \star *$ type num

pipe 661

ncells

nodes

ichf

ich
0

radin 1

$0.0000 e+00$

toutv1

$3.0000 e+02$

iconc
0
th1

$7.1120 e-03$
$7.23900 \mathrm{e}+05 \quad 7.23900 \mathrm{e}+05 \quad 7.23900 \mathrm{e}+05 \mathrm{~s}$

$7.23900 e+05 \quad 7.23900 e+05 \quad 7.23900 e+05 s$

$7.23900 \mathrm{e}+05 \quad 7.23900 \mathrm{e}+05 \quad 7.23900 \mathrm{e}+05 \mathrm{~s}$

$7.23900 e+05 \quad 7.17570 e+05 \quad 7.04910 e+05 e$

$3.60600+00$

$1.5405 \mathrm{e}-01 \mathrm{e}$

r15 le

id ctitle
$661 \$ 661 \$$ RHR - cold leg, pt 2

jun1 jun2 epsw

$661 \quad 662 \quad 5.0000 e-05$

iacc

ipow

$0.0000 e+00$

houtv1

$0.0000 e+00$

toutl1

$3.0000 e+02$ 
$*$

* vol * $1.8639 \mathrm{e}-02 \mathrm{e}$

* fa * f $1.8639 \mathrm{e}-02 \mathrm{e}$

* fric * $* 0.25$ $1.0000 e-08 e$

* ha * E $1.5405 e-01 e$

* nff * $E$ 1e

*alp * f $0.0000 e+00 e$

*v1 * f $0.0000 e+00 e$

*vv *f $0.0000 e+00 \mathrm{e}$

* $t 1 * \mathrm{f} 3.1315 \mathrm{e}+02 \mathrm{e}$

*tv *f $3.1315 \mathrm{e}+02 \mathrm{e}$

* p * f $6.98583 e+05 e$

* pa * f $0.0000 e+00 e$

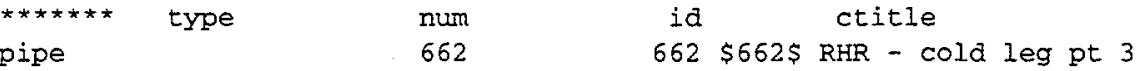

* cold leg from heat exchanger exit ncelis nodes leg from heat exchanger exit

$\begin{array}{rrrrr}\text { nodes } & \text { jun1 } & \text { jun2 } & \text { epsw } \\ 8 & 0 & 662 & 663 & 5.0000 e^{-05} \\ \text { ichf } & \text { iconc } & \text { iacc } & \text { ipow } & \\ 0 & 0 & 0 & 0 & \text { toutl } \\ \text { radin } & \text { th } & \text { houtl } & \text { houtv } & \text { touts }\end{array}$

$7.7026 \mathrm{e}-02$

7.1120 e- 03

$0.0000 e+00$

$0.0000 \mathrm{e}+00$

$3.0000 e+02$

$3.0000 e+02$

* dx * r5 $1.3800 e+00$ r3 1.7467e+00e

* vol * r5 2.5722e-02 r3 $3.2556 \mathrm{e}-02 \mathrm{e}$

* fa * $\mathrm{f} 1.8639 \mathrm{e}-02 \mathrm{e}$

* fric * r2 $1.0000 e-08$

* fric * r2 $1.0000 e-08$

$0.0833 \quad r 21.0000 e-08 \quad 0.25 s$

* elev * r5 $5.5560 e+00$

* hd * f $1.5405 \mathrm{e}-01 \mathrm{e}$

* nff *f le

*alp * $* 0.0000 e+00 e$

$\star \mathrm{vl} * \mathrm{f} \quad 0.0000 \mathrm{e}+00 \mathrm{e}$

*vv * E $0.0000 e+00 e$

*t *f $3.3215 e+02 e$

*tv. *f $3.3215 \mathrm{e}+02 \mathrm{e}$

* $\quad$ * $6.98583 e+05$

* $\mathrm{p} * 6.98583 \mathrm{e}+05$

* pa *f $0.0000 e+00 e$

*

$6.98583 e+05 \quad 6.98583 e+05 \quad 6.98583 e+05 s$

$7.06860 \mathrm{e}+05 \quad 7.15595 \mathrm{e}+05 \quad 7.26937 \mathrm{e}+05 \mathrm{e}$

pipe 653

* ncelis nodes

$4.6987 e+00 \quad 3.7940 e+00 \quad 2.6193 e+00 e$

* ich

radin1

$7.7026 e-02$

toutv1

$3.0000 e+02$

$*$

$\star \mathrm{dx} \quad * \quad 1.0000 e+00 \mathrm{e}$

* vol * $\quad 1.8639 e-02 e$

* fa * f $1.8639 \mathrm{e}-02 \mathrm{e}$

* fric * 0.25

* elev * f $2.0320 e+00 e$

$663 \$ 563 \$$ RHR - cold leg, pt 4 jun1 jun2 epsw

$663 \quad 664 \quad 5.0000 e-05$

iacc ipow

0

houtl1 houtv1 toutl1

$0.0000 e+00 \quad 0.0000 e+00 \quad 3.0000 e+02$ 
* ha * $\quad$ 1.5405e-01e

$*$ nff $* 1$ a $1 e$

*alp *E $0.0000 e+00 e$

$* \mathrm{vl} * E \quad 0.0000 e+00 e$

$* v V * E \quad 0.0000 e+00 e$

*tI *E $3.307 e+02 e$

$* t v * E \quad 3.307 e+02 e$

$* p \quad * E 7.32609 e+05 e$

$* \mathrm{pa} \quad * E \quad 0.0000 e+00 e$

$\star$

*

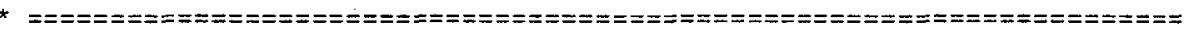

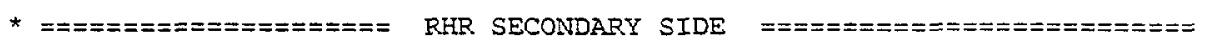

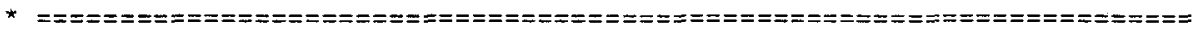

\begin{tabular}{|c|c|c|c|c|c|c|c|}
\hline & type & num & & id & & ctitle & \\
\hline & & 672 & 672 & $\$ 672 \$$ & Loop ? & $I$ RHR - HX & sec Eill \\
\hline & jun1 & ifty & & Loff & & & \\
\hline & 672 & 8 & & 2 & & & \\
\hline & iftr & ifsv & & $n f t b$ & & nfsv & nfxf \\
\hline & 101 & 1 & & 3 & & 0 & 0 \\
\hline & twtold & $r \operatorname{fin} x$ & & concin & & felv & \\
\hline & $0.0000 e+00$ & $1.0000 e+20$ & 0.00 & $00 e+00$ & & $9560 e+00$ & \\
\hline & dxin & volin & & alpin & & vlin & tlin \\
\hline & $5.0000 e-01$ & $4.7055 e-02$ & 0.00 & $00 e+00$ & & $.0000 e+00$ & $3.1100 \mathrm{e}+02$ \\
\hline & pin & pain & & flowin & & voin & tvin \\
\hline
\end{tabular}

$2.0000 e+06$

$0.0000 e+00$

$2.5220 \mathrm{e}+01$

$0.0000 e+00$

$3.1100 e^{0}+02^{-\cdots}$

* flowoff

$4.1682 e+06$

vloff

$0.0000 e+00$

vvsc1

$1.0000 e+00$

$1.0000 e+00$
alpoff

$0.0000 e+00 \quad 0.0000 e+00$

*

* vmtb * $* 0.0000 e+00$

*vmtb * $6.0000 e+01$

$2.5220 \mathrm{e}+01 \mathrm{~s}$

* vmtb * $1.0000 e+04 \quad 2.5220 e+01 e$

*

$\begin{array}{lcc}\star \star \star \star \star \star \star & \text { type } & \text { num } \\ \text { pipe } & - & 671\end{array}$

pipe nceils

* $\quad$ ichf

1

radin

$0.0000 e \div 00$

toutv

$3.0000 e+02$ nodes

iconc

0

$0.0000 e+00.0 .0000 e+00$

$0.0000 e+00$

$0.0000 e+00$

*

* dx *f $9.9000 e-01 e$

* vol *f $9.3466 \mathrm{e}-02 \mathrm{e}$

* fa *f $9.4411 e-02 e$

* fric *f $1.0000 e-08 e$

* elev * f $2.9560 e+00 e$

* hd *f $3.5419 e-02 \mathrm{e}$

$* \operatorname{nff} * \quad-1 \quad r 3 \quad 1 \quad-1 e$

$*$ alp *f $0.0000 e+00 e$

*v1 *f $2.686 \mathrm{e}-01 \mathrm{e}$

* vv *E 2.686e-01e

$* t 1 *$ E $3.1100 e+02 e$

$* t v * f 3.1100 e+02 e$
$671 \$ 671 \$$ Loop 1 RHR - HX sec part 2 jun1 jun2 epsw $672 \quad 673$ ipow $1.0000 e-05$ iacc 0 0
toutl $3.0000 e+02$ 
Report: Section:

Date:

Page:
WSRC-TR-98-0053

Appendix A

$07 / 14 / 98$

41 of 96

TRAC BLANKET SYSTEM MODEL

$2.00002 e+06 e$

$*$ p $\quad * \quad 2.00011 e+06$
$*$ pa $\quad * 0000 e+00 e$

*

$\star$

$\star * * * * * *$ type num

break 673

* jun1 ibty

673

ibty

$2.00008 e+06 \quad 2.00005 e+06$

* dxin

5.0000 e-01

pain

volin

$4.7055 e-02$

concin

$0.0000 e+00$

$0.0000 e+00$

id ctitie

$673 \$ 673 \$$ Loop 1 RHR - HX sec break

isat ioff

00

$0.0000 \mathrm{e}+00 \quad 3.1800 \mathrm{e}+02$

rbmx

$1.0000 e+20$

poff

$2.0000 e+06$

belv

*

*

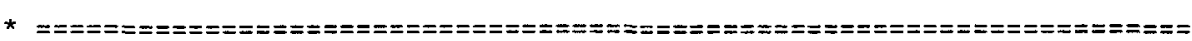

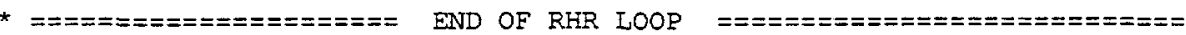

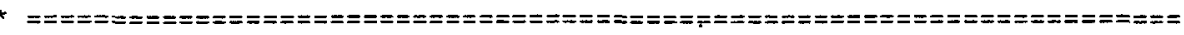

*

*

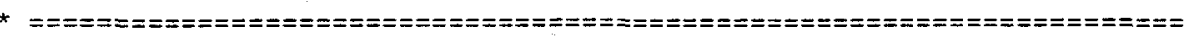

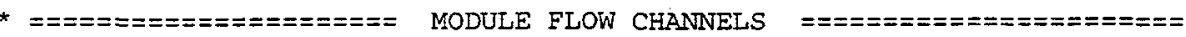

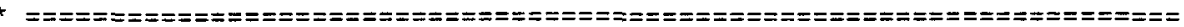

*

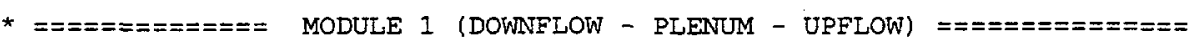

* Pipe connection from Cold Leg Fixed Header

$\begin{array}{lrrrrr}* \star * * * * & \text { type } & \text { num } & \text { id } & \text { ctitle } & . \\ \text { pipe } & & 454 & 454 \$ 454 \$ & \$ \text { Pipe conn. header } & \\ * & \text { ncells } & \text { nodes } & \text { junl } & \text { junz } & \text { epsw } \\ & 7 & 0 & 454 & 380 & 1.0000 \mathrm{e}-05 \\ * & \text { ichf } & \text { iconc } & \text { iacc } & \text { ipow } & \\ & 1 & 0 & 0 & 0 & \text { toutl }\end{array}$

$\begin{array}{lllll}9.5250 e-02 & 1.1130 e-02 & 0.0000 e+00 & 0.0000 e+00 & 3.0000 e+02\end{array}$

* toutv

$3.0000 e+02$

*

$* \mathrm{dx} \quad * \mathrm{r} 2 \quad 6.2500 \mathrm{e}-01$

$7.6200 e-01 \times 3 \quad 1.2500 e+00$

$4.5000 \mathrm{e}-01 \mathrm{e}$

* vol

* r2 $3.8082 \mathrm{e}-02$

$4.6429 \mathrm{e}-02$ r3 $7.6164 \mathrm{e}-02$

$2.7419 e-02 e$

* Ea.

* f $6.0931 \mathrm{e}-02 \mathrm{e}$

* fric * $0.2500 e+00$

* Eric * $0.0100 e+00$

$0.0100 e+00$

$0.0100 e+00$

$0.1700 \mathrm{e}+00$

$0.1700 e+00 \mathrm{~s}$

* elev

$1.7772 e+00$

$1.7772 \mathrm{e} \div 00$

$1.0000 \mathrm{e}-02$

* r3 $1.0152 \mathrm{e}+00$

* f $1.9050 e-01 \mathrm{e}$

* hd

$7.9020 e-01 e$

$1.3962 \mathrm{e}+00 \mathrm{~s}$

$1.0000 e-08 \mathrm{e}$

* nff

* 1 r5 1

*alp *f $0.0000 e+00 e$

*VI *f $9.046 e+00 e$

*v *f $9.046 e+00 e$

$* t 1 \quad * \mathrm{f} \quad 3.2315 \mathrm{e}+02 \mathrm{e}$

$\star t V * f \quad 3.2315 e+02 e$

$\star p \quad * 6.83083 e+05$

$\star \mathrm{p} \quad * \quad 6.67122 \mathrm{e}+05$

$*$ pa $\quad$ f $0.0000 e+00 e$

$6.81142 e+05$

$6.76253 e+05$

$6.63647 e+05$

$6.63337 \mathrm{e}+05 \mathrm{e}$

$6.70598 \mathrm{e}+05 \mathrm{~s}$

* Decoupler upper Plenum

$* \star \star \star * \star * *$ type

plenum 80

$80-i c$

ctitle

$\begin{array}{rr}\text { * } & \text { iconc } \\ 3 & 0\end{array}$

juns?

$\$ 80 \$$ Dec UpperPI

juns2 
*

* juncs * 380 s

* juncs * $80 \mathrm{~s}$

*Inlet Hd to UPl

* juncs * $841 e$

*UP1 to Decoupler

*Valve connected to Cavity vessel

* dx * r2 1.1520e-01 5.7600e-02e

* vol * 7.0193e-03e

* elev * $5.0750 \mathrm{e}-01 \mathrm{e}$

*alp * $0.0000 \mathrm{e}+00 \mathrm{e}$

$* t 1 * 3.2315 e+02 e$

* tv * $\quad * .2315 e+02 e$

$* \mathrm{p} \quad * \quad 7.05858 \mathrm{e}+05 \mathrm{e}$

$* \mathrm{pa} * 0.0000 \mathrm{e}+00 \mathrm{e}$

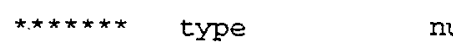

pipe 375

* ncelis nodes

* $\begin{array}{r}5 \\ \text { ichf }\end{array}$

* radin

$7.6200 e-02$

toutv

id ctitle

$375 \$ 375 \$$ Pipe conn. tee-plenum 370

jund jun2 epsw

$80 \quad 307$

$1.0000 e-05$

$3.0000 e+02$

*

$* \mathrm{dx} \quad$ *

* vol $4.5000 e-01$

* * $\quad 2.7419 e-02$

* Ea *

* Eric * $1.0000 \mathrm{e}-08$

* fric * $0.0100 e+00$

* elev * $2.2500 \mathrm{e}-01$

* elev * $-3.5000 e+00 e$

* ha * E $1.9050 \mathrm{e}-01 \mathrm{e}$

$*$ nff * $1 \quad r 41$

*alp * $f \quad 0.0000 e+00 e$

* v1 *f $9.046 \mathrm{e}+00 \mathrm{e}$

$* \mathrm{vv} * \mathrm{f} 9.046 \mathrm{e}+00 \mathrm{e}$

$\star t 1 * f \quad 3.2315 e+02 e$

$*$ tv * $\pm 3.2315 e+02 e$

$* \mathrm{p} * \quad 6.67432 \mathrm{e}+05$

* p * $\quad$ * $6.92798 \mathrm{e}+05 \mathrm{e}$

$\star \mathrm{pa} \quad$ * $\quad 0.0000 \mathrm{e}+00 \mathrm{e}$

$r 21.0000 e+00 \quad r 21.0000 e+00 e$

r2 $6.0931 \mathrm{e}-02$ r2 $6.0931 \mathrm{e}-02 \mathrm{e}$

$0.0100 e+00$

$0.0100 e+00$

$0.0100 \mathrm{e}+00 \mathrm{~s}$

$0.1000 \mathrm{e}+00 \mathrm{e}$

$-5.0000 e-01 \quad-1.5000 e+00 \quad-2.5000 e+00 s$

$-1 e$

*

* Decoupler Upper Plenum

******* type

plenum 370

* npljn iconc

$6.72278 e+05 \quad 6.79118 e+05 \quad 6.85958 e+05 s$

tout I

$3.0000 e+02$

2

0

370 ctitle

juns1

$\$ 370 \$$ Dec UpperPI

*

* juncs * $307 \mathrm{~s}$ *Inlet Hd to UPI

* juncs * $308 \mathrm{e}$ *UP1 to Decoupler

*

$* \mathrm{dx} \quad * \pm 2.0320 \mathrm{e}-01 \mathrm{e}$

$*$ vol * 5.0000e-01e

* elev * $-4.0170 e+00 e$

$* \operatorname{alp} * 0.0000 e+00 e$

$* t 1 * 3.2315 e+02 e$

$* t v * 3.2315 e+02 e$

$* \mathrm{p} \quad * \quad 6.94268 \mathrm{e}+05 \mathrm{e}$

* pa * $\quad$ pa.0000e+00e 
*

* Mocule 1 Downflow

$* * * * * *$ type num

pipe 360

$360 \$ 360 \$$ Decoupler water

* ncells nodes

* $\begin{array}{r}5 \\ 1\end{array} \quad$ ich

$0.0000 e+00$

$1.1130 e-02$

toutv1

jun1

308

jun2

309

$0 \quad$ racc

hout 11

$0.0000 \mathrm{e}+00$

$000 e+00$

tout II

$3.0000 e+02$

*

$\star \mathrm{dx} \quad * \quad 3.1980 e-01$

* vol * $6.3714 e-02$

* fa * £ $1.9923 e-01 e$

* fric * $1.3229 e+00$

* elev * $-4.2785 e+00$

* elev * $\quad-5.9624 e+00$

* hd *f 8.6117e-03e

$\star$ nff *f le

*alp *f $0.0000 e+00 e$

*vl *f $2.769 e+00 e$

$\star v v \quad$ t $2.769 e+00 e$

*ti *f $3.2315 e+02 e$

*tv *f $3.2315 e+02 e$

$* p \quad * \quad 6.85124 e+05$

* p * $\quad$ * $6.81981 \mathrm{e}+05 \mathrm{e}$

* pa *f $0.0000 e+00 e$

$6.84474 e+05 \quad 6.83638 e+05 \quad 6.82808 e+05 s$

$546.0960 \mathrm{e}-01 \mathrm{e}$

r4 $1.2145 \mathrm{e}-01 \mathrm{e}$

$54 \quad 1.0000 e-02 \quad 3.6840 e-01 e$

$-4.7432 e+00$

$-5.3528 \mathrm{e}+00 \mathrm{~s}$

$-6.5720 e+00 e$

$3.0000 e+02$

* Module 1 Mid-plenum

$* * * * * *$ type num

plenum 350

* npling iconc

350
0

id ctitle

$350 \$ 350 \$$ Down $P 1$ in water

$$
2
$$

juns1

juns2

* juncs * 309s *Dec to Down PI

* juncs * 310 * Down PI to Row1

$\star d x \quad * f \quad 7.6200 e-02 e$

* vol * $5.0000 e-01 e$

* elev * $-6.9149 e+00 e$

* alp * $0.0000 e+00 e$

$* t I * 3.2615 \mathrm{e}+02 \mathrm{e}$

$\star t v * 3.2615 \mathrm{e}+02 \mathrm{e}$

$* \mathrm{p} \quad * \quad 6.83934 \mathrm{e}+05 \mathrm{e}$

* pa * $\quad$ pa $0000 e+00 e$

* Module 1 Upflow

$\star \star \star \star \star \star *$ type num

pipe 300

$300 \quad 300 \$ 300 \$$ Rowl water

* ncells nodes

5

nodes

icone

id
300
jun1
310
iacc
0

id
300
jun1
310
iacc
0

hout1

jun2
320
ipow
0
houtv

epsw

$1.0000 \mathrm{e}-05$

radin

th

hout




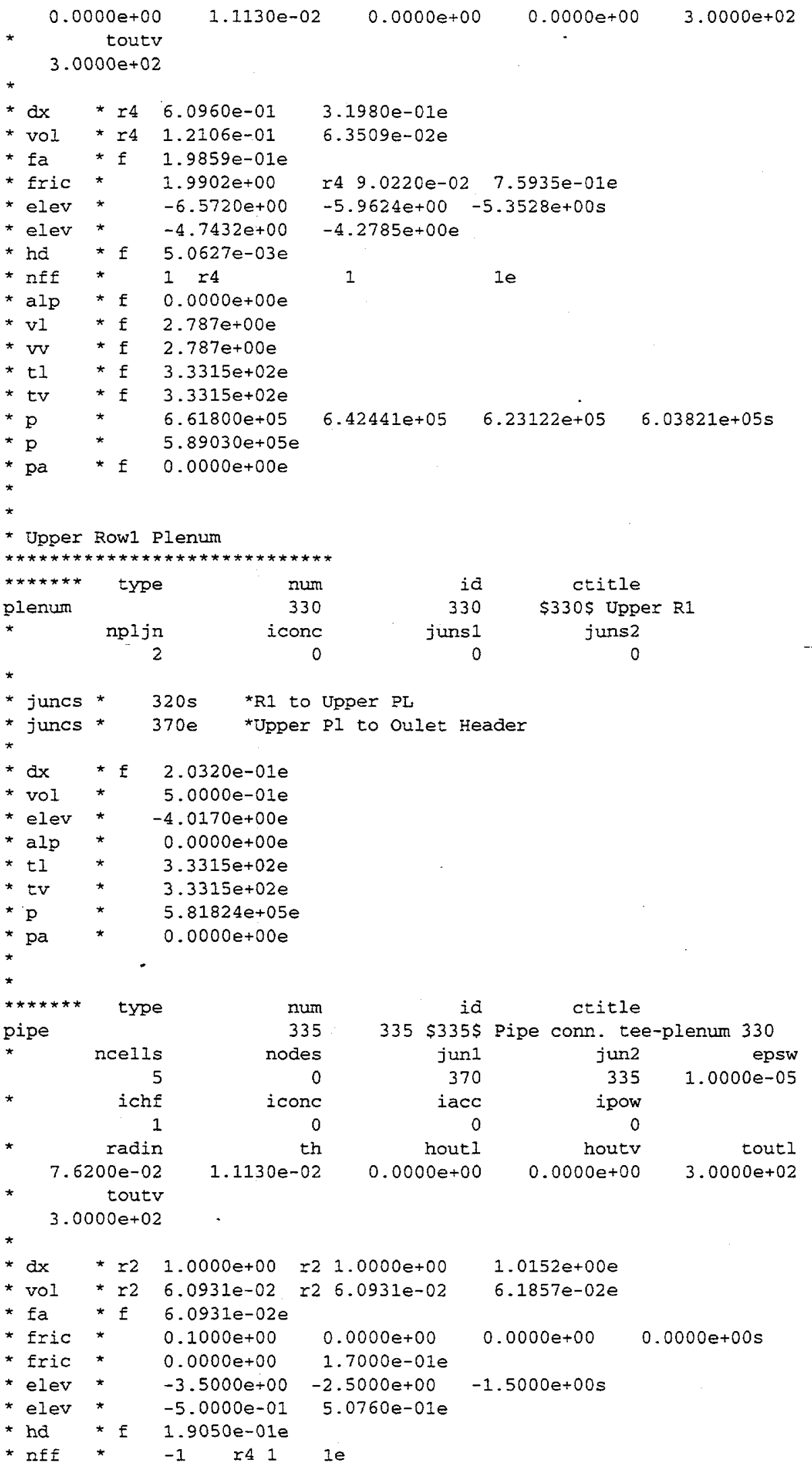


*alp *f $0.0000 e+00 e$

*v1 *f $9.094 e+00 e$

* vv *f $9.094 e+00 e$

*tI *f $3.3315 e+02 e$

*tv *f $3.3315 e+02 e$

$* \mathrm{p} \quad * \quad 5.10970 \mathrm{e}+05$

* $\mathrm{p} \quad * \quad 4.62494 \mathrm{e}+05 \mathrm{e}$

* pa *E $0.0000 \mathrm{e}+00 \mathrm{e}$

$4.98873 e+05 \cdot 4.86777 e+05 \quad 4.74682 e+05 s$

\author{
$(20873+05$
}

* Pipe connection from Hot Leg Fixed Header to tee 428 component

pipe

type

\title{
num
}

429

id ctitie

*

$$
\text { ncells }
$$

nodes

$429 \$ 429 \$$ Pipe conn. $428-340$

ichf

0

* $\quad$ ichf

radin

$9.5250 e-02$

$1.1130 e^{\text {th }}-02$

* toutv

0

th

$3.0000 e+02$

*

$* \mathrm{dx} \quad * \mathrm{r} 4 \quad 1.2500 \mathrm{e}+00 \mathrm{e}$

* vol * r4 $7.6164 \mathrm{e}-020$

* fa $* \mathrm{f} 6.0931 \mathrm{e}-02 \mathrm{e}$

* fric * $1.7000 e-01 r 31.0000 e-02$

* elev * $1.0152 e+00$ r2 $1.0152 e+00$

* há *f $1.9050 \mathrm{e}-01 \mathrm{e}$

* nff * 1 r3 1 1e

*alp *f $0.0000 e+00 e$

$* v I * f \quad 9.094 e+00 e$

$* v v * f \quad 9.094 e+00 e$

* EI *f $3.3315 e+02 e$

$* t v * f \quad 3.3315 e+02 e$

$* p \quad * \quad 4.47909 e+05$

*pa *f $0.0000 e+00 e$

$4.44446 e+05 \quad 4.40982 e+05 \quad 4.37518 e+05 e$

$0.5000 e+00 e$

$1.0152 \mathrm{e}+00 \mathrm{e}$

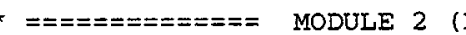

DOWNFLOW - PLENUM - UPFLOW)

* Module 2 Downflow

* 2nd Lateral Module

* Water Pipe Connection After Water Supply Manifold in Loop5

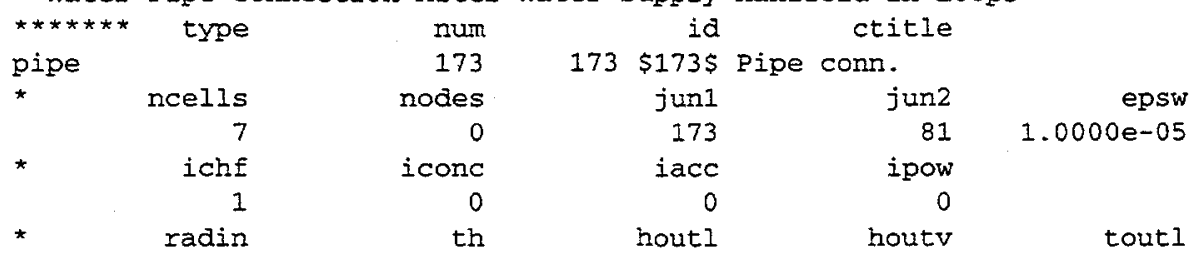

$6.0325 e-02$

$1.1130 \mathrm{e}-02$

$0.0000 e+00$

$0.0000 e+00$

$3.0000 e+02$

* toutv

$3.0000 e+02$

*dx * $d 2 \quad 6.2500 \mathrm{e}-01$

* dx * 4.5000e-01e

* vo1 * r2 $2.8583 \mathrm{e}-02$

* vol * $2.0579 \mathrm{e}-02 \mathrm{e}$

* fa *f $4.5732 \mathrm{e}-02 \mathrm{e}$

* fric * 2.5000 e-01

* fric * 1.0361e-01
$7.6200 \mathrm{e}-01 \times 3 \quad 1.2500 \mathrm{e}+00 \mathrm{~s}$

$3.4848 e-02 r 35.7165 e-02 s$

$5.1803 e-02$

$1.0361 e-01$
$1.0000 e-05$

tout 1

$3.0000 \mathrm{e}+02$ 


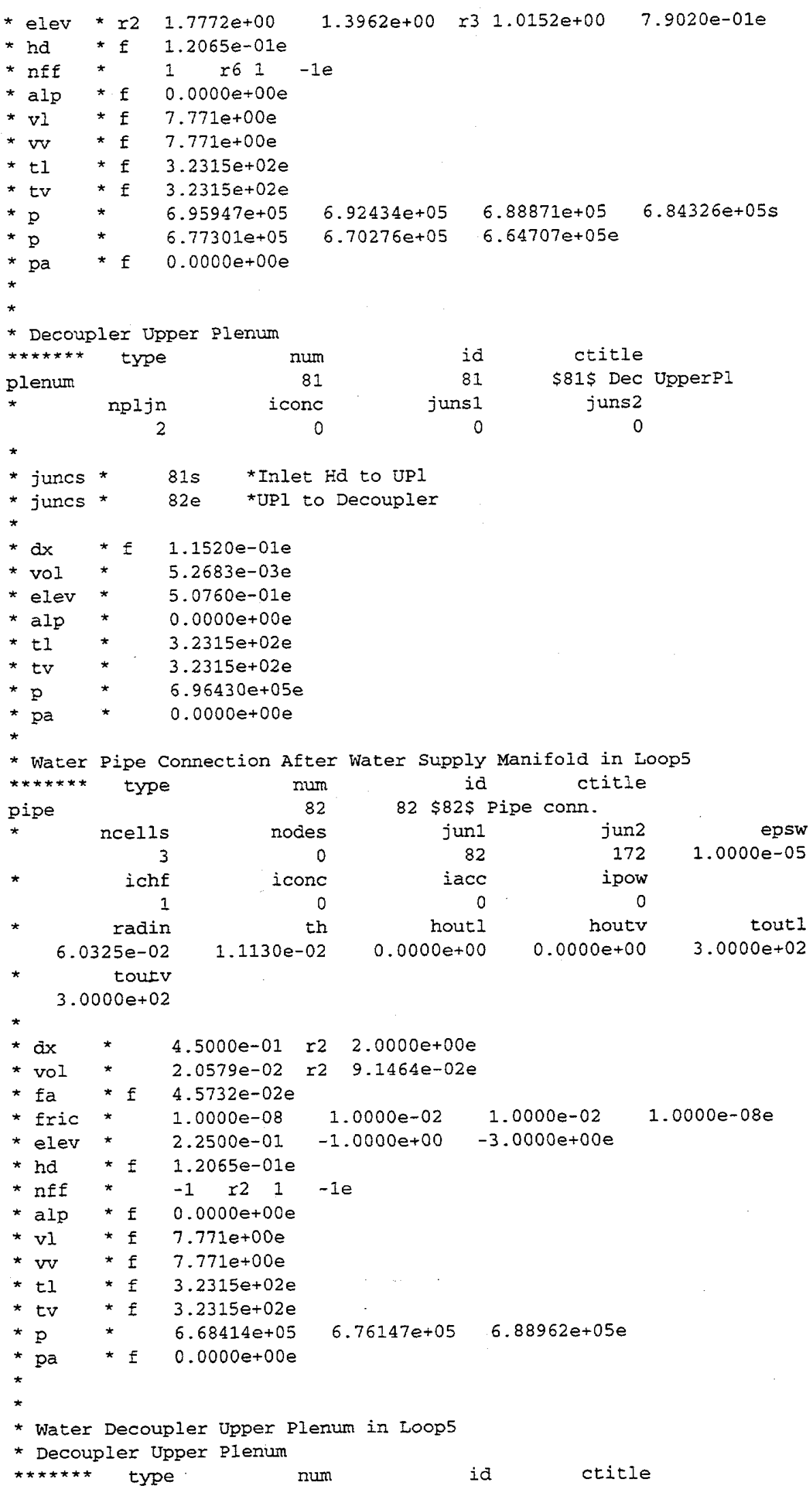




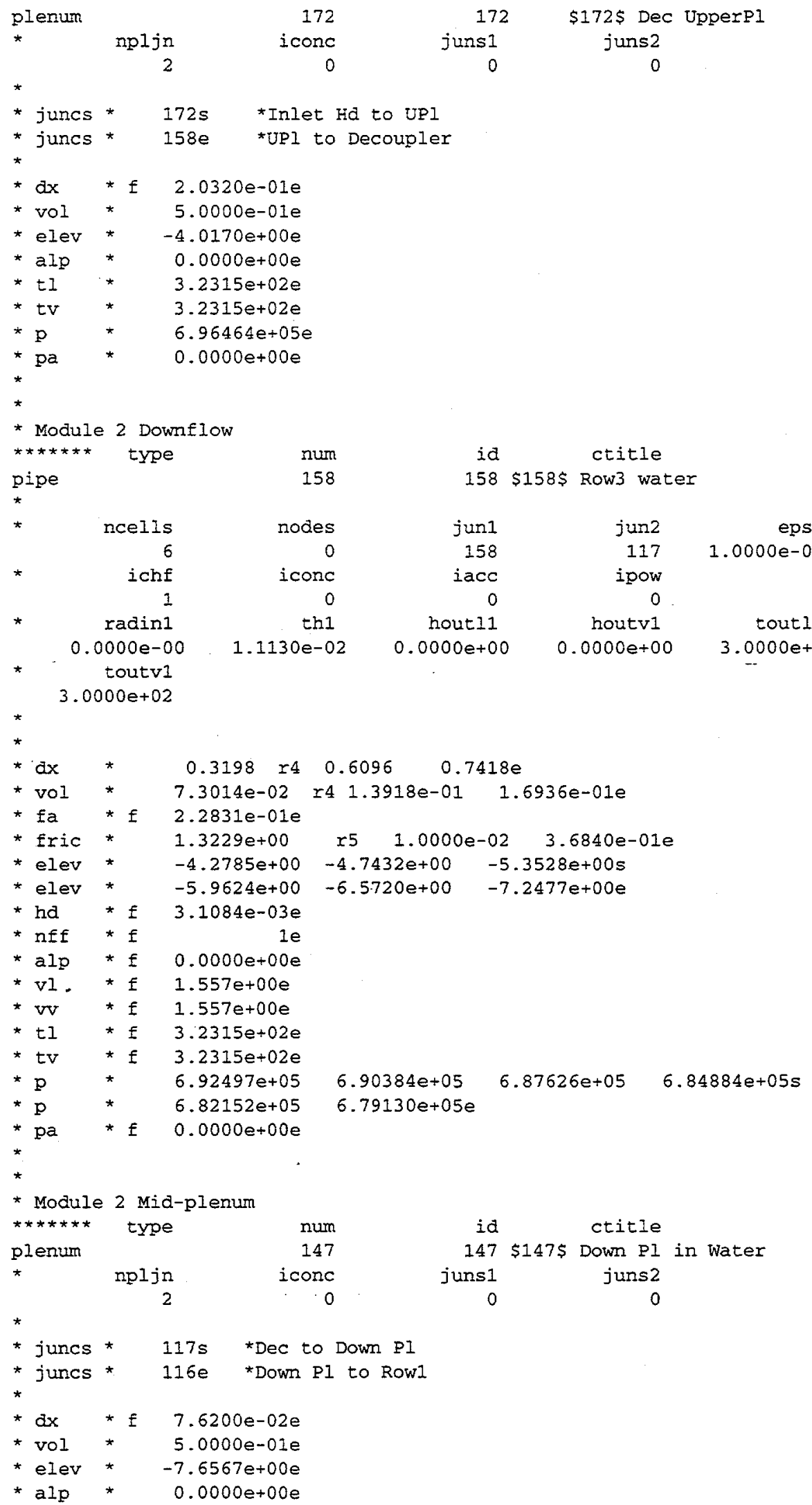


$* t 1 * 3.2615 e+02 e$

*tv * $3.2615 e+02 e$

* $\mathrm{p} * 6.78066 \mathrm{e}+05 \mathrm{e}$

* pa $\quad * \quad 0.0000 e+00 e$

*

$*$

* Module 2 upflow

$* * * * * * *$ type num

Page:

id ctitle
$102 \$ 102 \$$ Row2 water

jun1 jun2 epsw

$116 \quad 102 \quad 1.0000 e-05$

iacc ipow

pow

houtv

$0.0000 e+00$

$3.0000 e+02$

\title{
$0.0000 e-00$
}

$1.1130 e-02$

$0.0000 e+00$

$3.0000 e+02$

dx $\quad * \quad 0.7418 \quad r 4$

* vol * $\quad$ * $1.5081 e-01 r 4$

* fa *f 2.0330e-01e

* fric * $1.9902 e+00$

* elev * $\quad-7.2477 e+00$

* elev * $\quad-5.3528 e+00$

* ha *f $3.1838 \mathrm{e}-03 \mathrm{e}$

* nff *

*alp *f $0.0000 e+00 e$

$\star \mathrm{vl} * \mathrm{f} 1.750 \mathrm{e}+00 \mathrm{e}$

$* \mathrm{vv} * \mathrm{f} 1.750 \mathrm{e}+00 \mathrm{e}$

$* t 1 * \pm 3.3015 e+02 e$

*tv * $\mathrm{t} 3.3015 e+02 e$

$* \mathrm{p} * \quad 6.62665 \mathrm{e}+05$

* p * 5.95861e+05

* pa * f $0.0000 e+00 e$

\begin{abstract}
$6.44586 e+05$
$6.28313 e+05$

$6.12078 \mathrm{e}+0.5 \mathrm{~s}$
\end{abstract}

$5.83469 e+05 e$

$0.3198 \mathrm{e}$

$1.2393 e-01 \quad 6.5015 e-02 e$

r5 $9.0220 \mathrm{e}-02 \quad 7.5935 \mathrm{e}-01 \mathrm{e}$

$-6.5720 e+00 \quad-5.9624 e+00 s$

$-4.7432 e+00-4.2785 e+00 e$

* Water Upper Plenum in Loop5

* Upper Row1 Plenum

$\star * * * * * * * * * * * * * * * * * * * * * * * * * \star \star *$

$\begin{array}{lrrrr}* * * * * * * & \text { type } & \text { num } & \text { id } & \text { ctitle } \\ \text { plenum } & & 133 & 133 & \$ 133 \$ \text { Upper R1 } \\ * & \text { np1jn } & \text { iconc } & \text { juns } 1 & \text { juns2 } \\ & 2 & 0 & 0 & 0\end{array}$

* juncs * $102 \mathrm{~s} \quad$ *R1 to Upper PL

* juncs * 133 e *Upper PI to Oulet Header

* $d x \quad * f 2.0320 e-01 e$

* vol * $5.0000 e-01 e$

* elev * $-4.0170 e+00 e$

*alp * $\quad$ a $0.0000 e+00 e$

*t1 * $\quad$ * $3.3015 e+02 e$

* tv * $\quad$ * $3.3015 e+02 e$

* $\mathrm{p}$ * $5.76938 \mathrm{e}+05 \mathrm{e}$

* pa $\quad$ pa $0.0000 e+00 e$

$\star$

$\star$

* Water Pipe Before Water Return Manifold in Loop5

$\star * * * * *$ type num id ctitle

pipe $136 \quad 136 \$ 136 \$$ Pipe conn.

* ncelis nodes $\quad$ jun1 jun2 epsw 


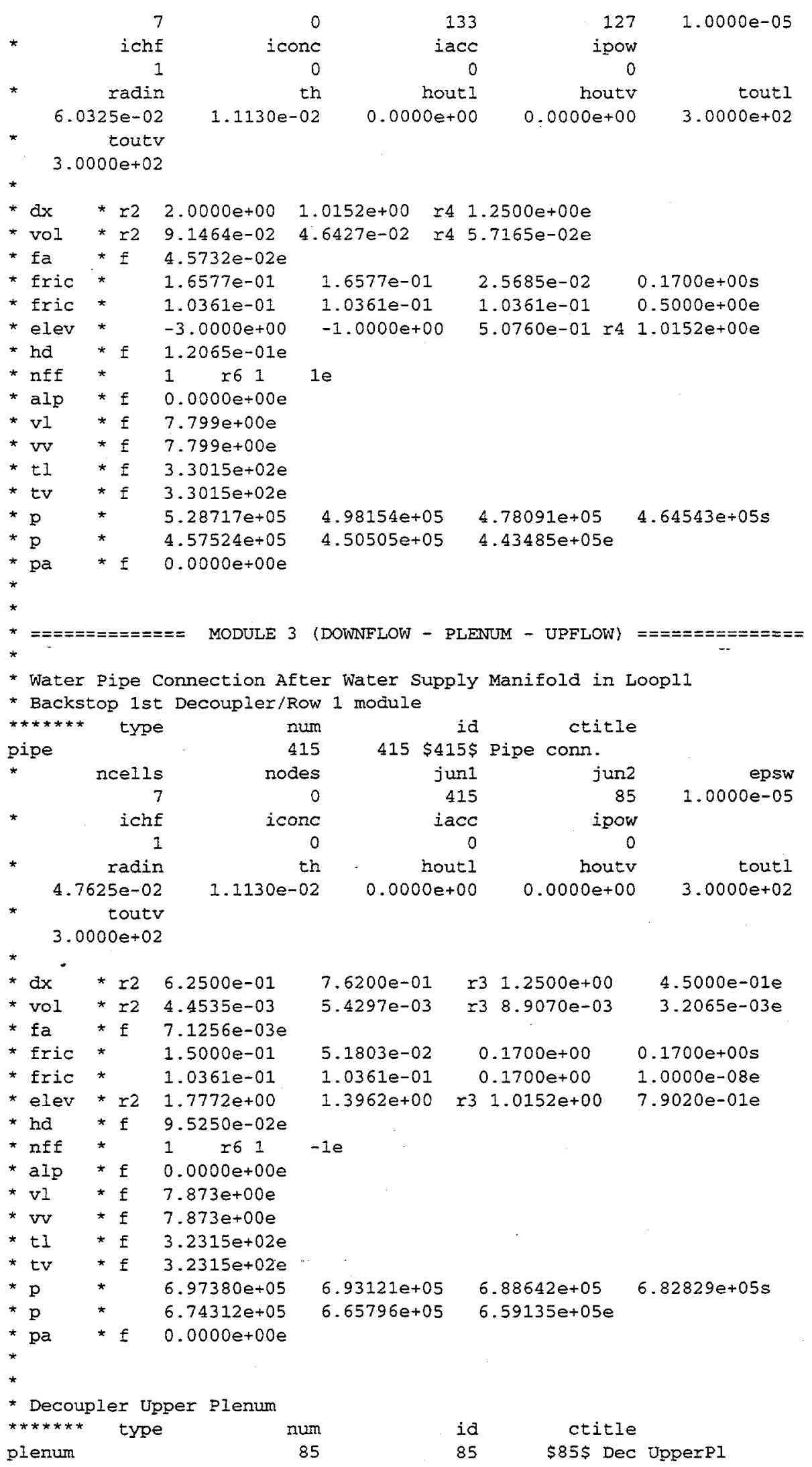




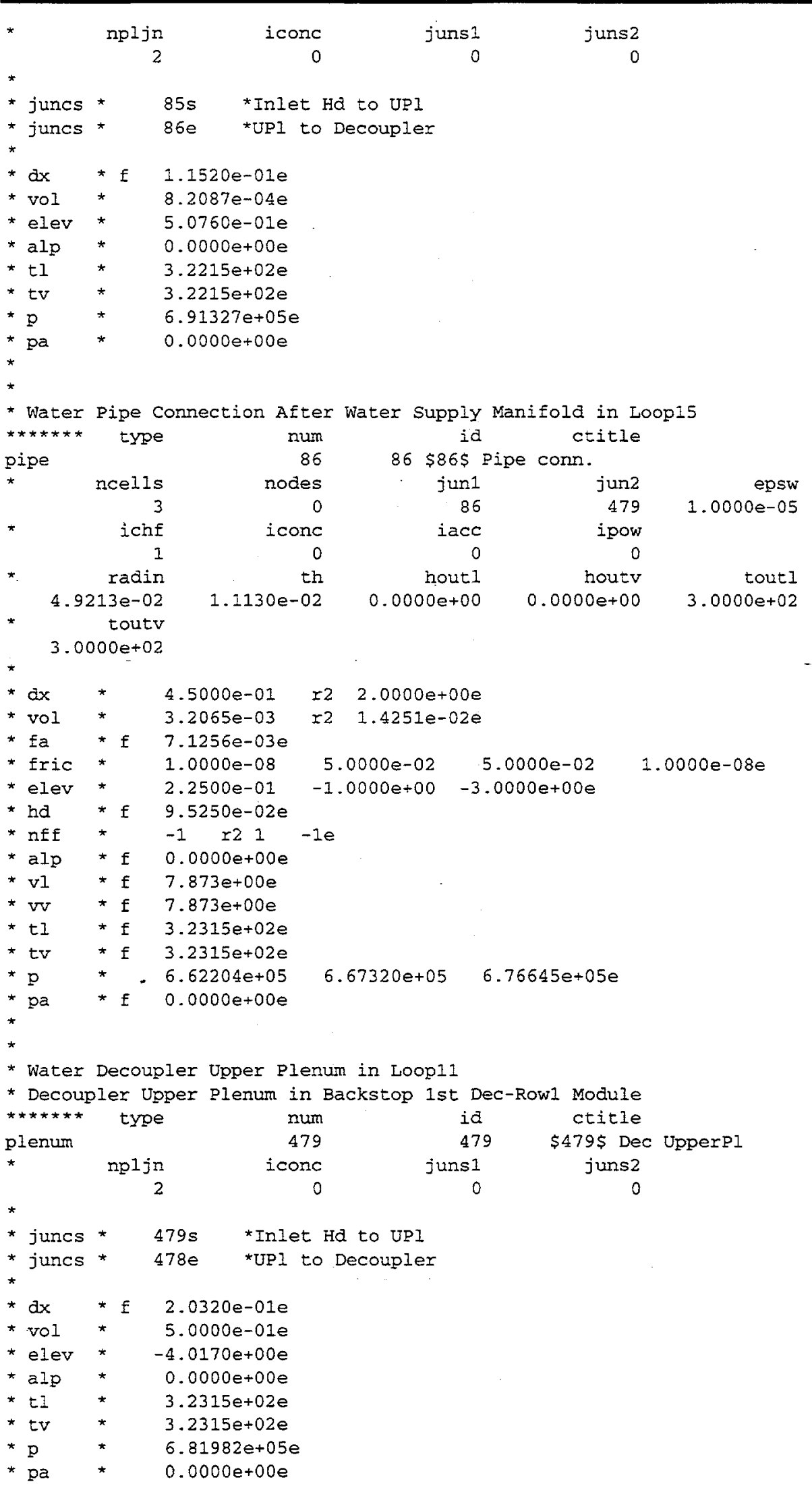


*

* Module 3 Downflow

$\star \star \star \star * \star *$ type num

pipe

*

* ncell

ich

radin 1

$0.0000 \mathrm{e}+00$

toutv1

$3.0000 e+02$

$\star$

*

* dx

* vol

* fa

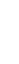

$3.1980 \mathrm{e}-01$

$8.8363 e-03$

f $2.7631 e-02 e$

* fric * $5.0000 e-01$

* elev * $\quad-4.2785 e+00$

* elev * $\quad-5.9624 e+00$

* ha *f $9.9388 e-03 e$

*nff *f

*alp * $f$

* v1 *f

$\star v v * f$

$\star t I$

$\star t v$

$\star$

* p

* pa

$\star$

$\star$

* Module 3 Mid-plenum

plenum

* npljo

* juncs * 418s *Dec to Down P1

* juncs * 426 e *Down Pl to Row1

*

*dx *f $7.6200 e-02 e$

* vol * 5.0000e-01e

* elev * $\quad-6.9149 e+00 e$

*a1p * $0.0000 e+00 e$

$\star t 1 \quad \star \quad 3.2615 \mathrm{e}+02 \mathrm{e}$

$\star \mathrm{tv} * \quad 3.2615 \mathrm{e}+02 \mathrm{e}$

* $\mathrm{p} \quad$ * $6.93228 \mathrm{e}+05 \mathrm{e}$

* pa * $0.0000 e+00 e$

$\star$

* Module 3 upflow

$\star * \star \star * * *$ type num

pipe

409

*

* ncells

5
$\operatorname{chf}$

* radin

* radin

nodes
0
iconc
0
th

478

id ctitle

$478 \$ 478 \$$ Decoupler water

r4 6.0960e-01e

$r 41.6844 \mathrm{e}-02 \mathrm{e}$

$\Upsilon 41.0000 e-02$

$-4.7432 e+00$

$-6.5720 e+00 e$

3. $6840 e-01 e$

$-5.3528 e+00 s$

$6.82180 e+05 \quad 6.84914 e+05 \quad 6.87650 e+05 s$ tout 11

$0.0000 e+00 \quad 3.0000 e+02$ epsw

$1.0000 e-05$

418

ipow

id
409
junt
426
iacc
0

ctitle

$418 \$ 418 \$$ Down PI in water

juns1 juns2

0 


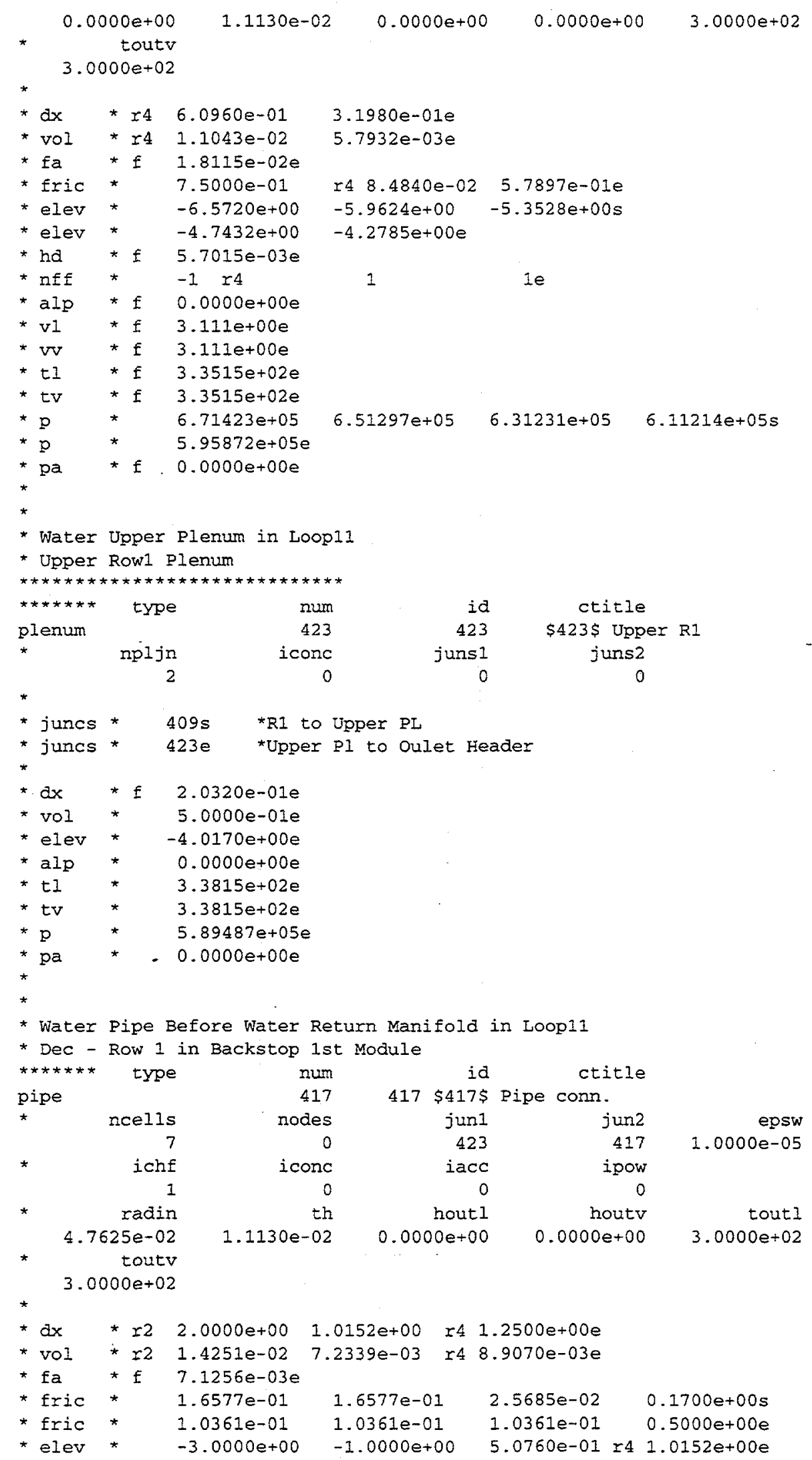


* hd * $f 9.5250 e-02 e$

* nff * 1 r6 1 1e

*alp *E $0.0000 e+00 e$

*VI * $\mathrm{vi} 7.936 e+00 \mathrm{e}$

$* \mathrm{vv} * \mathrm{f} 7.936 e+00 \mathrm{e}$

$* t+f \quad 3.3815 e+02 e$

*tv *f $3.3815 e+02 e$

$* \mathrm{p} * 5.39006 \mathrm{e}+05$

$* \mathrm{p} * \quad 4.61013 \mathrm{e}+05$

$*$ pa $* £ 0.0000 e+00 e$

$5.06154 e+05$

$4.52510 e+05$

$4.84458 \mathrm{e}+05$

$4.44007 e+05 e$

$4.69517 e+05 s$

* $=============$ M MODULE 4 (DOWNFLOW - PLENUM - UPFLOW) $===============$

* Water Pipe Connection After Water Supply Manifold in Loop12

* Water Pipe Connection in 2nd Layer Module Backstop

\begin{tabular}{|c|c|c|c|c|c|}
\hline & type & num & id & ctitle & \\
\hline & & 485 & $485 \$ 485 \$$ & Pipe conn. & \\
\hline * & ncells & nodes & jun1 & jun2 & epsw \\
\hline & 7 & 0 & 485 & 87 & $1.0000 e-05$ \\
\hline * & $i \operatorname{chf}$ & icone & jacc & ipow & \\
\hline & 1 & 0 & 0 & 0 & \\
\hline * & radin & th & hout I & houtv & toutl \\
\hline & $\begin{array}{r}6.8265 e-02 \\
\text { touty }\end{array}$ & $1.1130 \mathrm{e}-02$ & $0.0000 e+00$ & $0.0000 e+00$ & $3.0000 e+02$ \\
\hline
\end{tabular}

$3.0000 e+02$

$\star d x \quad *$ r2 $6.2500 e-01$

* vol * r2 $9.1500 e-03$

* fa $*$ f $1.4640 e-02 e$

* Eric * $1.5000 e-01$.

* Exic * $1.0361 e-01$

* elev * r2 $1.7772 e+00$

* ha *f $1.3653 \mathrm{e}-01 \mathrm{e}$

* nff * 1 ro 1 - 1 e

*alp *f $0.0000 e+00 e$

$* \mathrm{vl} * \mathrm{f} \quad 6.686 \mathrm{e}+00 \mathrm{e}$

$* \mathrm{vv} * \mathrm{f} \quad 6.686 \mathrm{e}+00 \mathrm{e}$

$* t I * \mathrm{f} 3.2315 \mathrm{e}+02 \mathrm{e}$

*tv *f $3.2315 e+02 e$

*p. * $7.08492 \mathrm{e}+05$

* $p \quad * \quad 6.97669 e+05$

*pa *f $0.0000 e+00 e$

$7.6200 e-01$ r3 $1.2500 e+00$

$4.5000 \ddot{e}-01 \mathrm{e}$

$1.1156 \mathrm{e}-02$ r3 $1.8300 \mathrm{e}-02$

$6.5880 e-03 e$

$5.1803 e-02$

$0.1700 e+00$

$0.1700 e+00 s$

$1.0361 \mathrm{e}-01$

$0.1700 \mathrm{e}+00$

$1.0000 \mathrm{e}-08 \mathrm{e}$

$1.3962 e+00$

$r 31.0152 e+00$

$7.9020 e-01 e$

$7.06076 e+05$

$7.04606 e+05$

$7.02499 e+05 s$

$6.92839 e+05$

$6.89536 e+05 e$

$\star$

$\star$

* Decoupler upper Plenum

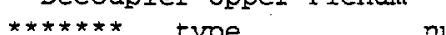

plenum type 87

* npljn iconc

id

87

juns 1

ctitle

2

0

$\$ 87 \$$ Dec UpperP1

juns2

juncs * $87 \mathrm{~s}$ *Inlet Ha to UPI

* juncs * 88 e *UPl to Decoupler

* dx * E $1.1520 e-01 e$

* vol * $1.6865 e-03 e$

* elev * $5.0760 \mathrm{e}-01 \mathrm{e}$

*alp * $\quad$ a $0.0000 e+00 e$

*tl * $\quad$ t $3.2215 e+02 e$

* tv * $3.2215 \mathrm{e}+02 \mathrm{e}$

* $\mathrm{p}$ * $7.13813 \mathrm{e}+05 \mathrm{e}$

* pa * $0.0000 e+00 e$ 


$$
\star
$$

ncelis

$$
\text { ia }
$$$$
\text { ctitie }
$$

pipe

88

$88 \$ 88 \$$ Pipe conn

jun1 jun2

$88 \quad 489$

iacc

iconc

1

th

1. 1130 e-02

$0.0000 e+00$

hout I

ipow

epsi

epsw
$00 e-05$

4. $9213 e-02$

touts

$3.0000 e+02$

$\star$

* vol * $6.5880 \mathrm{e}-03$

* fa * $\quad$ t $1.4640 \mathrm{e}-02 \mathrm{e}$

* Eric * $1.0000 e-08$

* elev * $2.2500 e-01$

* ha * $\pm 1.3653 e-01 e$

* nff * $\begin{array}{lllll}1 & -1 & \text { r2 } & 1 & -1 e\end{array}$

*alp * $\quad$ a $0.0000 e+00 e$

*VI * $6.686 \mathrm{e}+00 \mathrm{e}$

* vV * $\quad$ f $6.686 \mathrm{e}+00 \mathrm{e}$

*tI*f $3.2315 e+02 e$

*tv *f $3.2315 e+02 e$

* $\mathrm{p}$ * $6.93871 \mathrm{e}+05$

* pa * $\quad 0.0000 \mathrm{e}+00 \mathrm{e}$

$12 \quad 2.0000 \mathrm{e}+00 \mathrm{e}$

$r 2 \quad 2.9280 e-02 e$

5.0000 e-02 $\quad 5.0000 e-02$

$-1.0000 \mathrm{e}+00 \quad-3.0000 \mathrm{e}+00 \mathrm{e}$

houtv

$0.0000 e+00$

$3.0000 e+02$

toutl 54 of 96

* Water Decoupler Upper Plenum in Loop12

* Decoupler Upper Plenum in 2nd Layer Backstop

$\begin{array}{llll}* * * * * * * & \text { num } & \text { id } & \text { ctitle } \\ \text { plenum } & 489 & 489 & \$ 489 \$ \text { Dec }\end{array}$

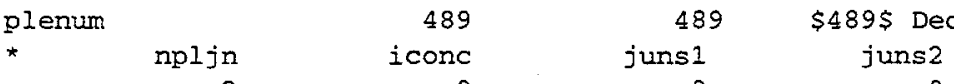

$\begin{array}{rrrr}2 & 0 & 0 & 0\end{array}$

*

* juncs * $489 \mathrm{~s}$ *Inlet Hd to UPl

* juncs * 480 e $*$ UPl to Decoupler

*dx *E. $2.0320 \mathrm{e}-01 \mathrm{e}$

* vol * 5.0000e-01e

* elev * $-4.0170 e+00 e$

*alp * $0.0000 \mathrm{e}+00 \mathrm{e}$

*t1 * $\quad$ * $3.2315 \mathrm{e}+02 \mathrm{e}$

* tv * $3.2315 \mathrm{e}+02 \mathrm{e}$

*p * $\mathrm{p}$ * $7.24285 \mathrm{e}+05 \mathrm{e}$

* pa * $\quad$ * $0.0000 e+00 e$

*

$\star$

* Module 4 Downflow

$* * * * * * *$ type num

pipe $\quad 480$

id ctitie

* ncells

* ichf

nodes

1

iconc

radinl

$0.0000 e-00$

toutv1

$3.0000 e+02$

$-02$

1.1130 e-02

$0.0000 e+00$

$480 \$ 480 \$$ Row 2 water

jun1 jun2

480

iacc

hout 11

$0.0000 e+00$ 
* $d x$

* vol

*

$0.3198 \quad r 4$

0.6096

$0.7418 \mathrm{e}$

* fa

$1.1540 e-02$

* Eric *

* elev * $-4.2785 e+00$

$r 42.1997 e-02 \quad 2.6768 e-02 e$

r5 $7.3700 e-02 \quad 7.5000 e-01 e$

* elev * $\quad-5.9624 e+00$

* ha * f $3.1198 \mathrm{e}-03 \mathrm{e}$

* nff *f $1 e$

*alp * E 0.0000e+00e

*v1 * E $2.719 e+00 e$

*vv * E $2.719 e+00 e$

*t1 *E $3.3015 e+02 e$

*tv * E $3.3015 e+02 e$

* $\mathrm{p}$ * $7.11357 \mathrm{e}+05$

* $\mathrm{p}$ * $6.43890 \mathrm{e}+05$

* pa * f $0.0000 e+00 e$

$-4.7432 e+00 \quad-5.3528 e+00 s$

$-6.5720 e+00 \quad-7.2477 e+00 e$

$\star$

* Module 4 Mid-plenum

$* * * * * *$ type num

plenum 419

$6.97515 e+05 \quad 6.79536 e+05 \quad 6.61680 e+05 s$

$6.24228 e+05 e$

55 of 96

*

* juncs * 419s *Dec to Down Pl

* juncs * 487 e *Down Pl to Rowl

* dx *E $7.6200 e-02 e$

* vol * 5.0000e-01e

* elev * $\quad-7.6567 e+00 e$

*alp * $0.0000 e+00 e$

* II * $\quad * 3.3215 e+02 e$

*tv * $\quad$ * $3.3215 e+02 e$

*p * $\mathrm{p} 6.13407 \mathrm{e}+05 \mathrm{e}$

* pa * $\quad$ pa $0000 e+00 e$

$\star$

$\star$

* Module 4 Upflow

$\star \star * * \star * *$ type num

pipe $\quad 412$

id ctitle

$412 \$ 412 \$$ Rowl water

* ncells nodes

6

6

junl

487

id ctitle

$419 \$ 419 \$$ Down $\mathrm{Pl}$ in water

juns 1

juns 2

0

0

* 1

iconc

iacc

0

hout 1

$0.0000 e+00$

1. 1130 e-02

$0.0000 \mathrm{e}+00$

jun2

412

ipow

$1.0000 e-05$

* toutv

3. $0000 e+02$

$\star$

* dx *

* vol

* fa

*

0.7418

$r 4$

0.6096

$0.3198 \mathrm{e}$

* Eric

* $\mathrm{f}$

5.1601e-02 rs

4. $2405 e-02$

$2.2246 e-02 e$

* elev

$6.9562 e-02 e$

r5 $9.0890 e^{-02} \quad 7.2777 e-01 e$

* elev

* ha *

$-7.2477 e+00$

$-6.5720 e+00$

$-5.3528 e+00$

$-4.7432 e+00$

$-5.9624 \mathrm{e}+00 \mathrm{~s}$

* nff

$5.1537 e-03 e$

*alo * f $0.0000 e+00 e$

* vl * f $1.422 \mathrm{e}+00 \mathrm{e}$ 
* vv *f $1.422 e+00 e$

*t t $\quad$ f $3.4315 e+02 e$

$* t v * f 3.4315 e+02 e$

$* \mathrm{p} * \quad 6.04924 \mathrm{e}+05$

$* \mathrm{p} \quad * \quad 5.65735 \mathrm{e}+05$

$*$ pa $\quad * \pm 0.0000 e+00 e$

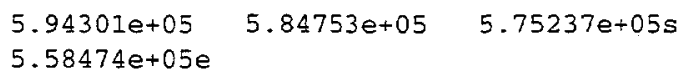

*.

* juncs * 412s *R1 to Upper PL

* juncs * 483 e *Upper PI to oulet Header

$$
\text { * }
$$

* dx *f $2.0320 e-01 e$

* vol * 5.0000e-01e

$*$ elev * $-4.0170 e+00 e$

*alp * $0.0000 e+00 e$

$*$ ti * $\quad 3.4615 \mathrm{e}+02 \mathrm{e}$

$*$ tv * $3.4615 \mathrm{e}+02 \mathrm{e}$

$* \mathrm{p} \quad * \quad 5.54709 e+05 e$

$\star \mathrm{pa} * 0.0000 \mathrm{e}+00 \mathrm{e}$

*

*

* Water Pipe Before Water Return Manifold in Loop12

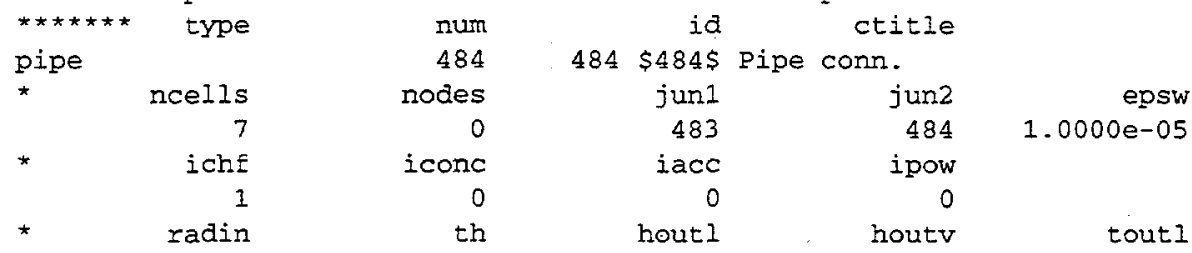

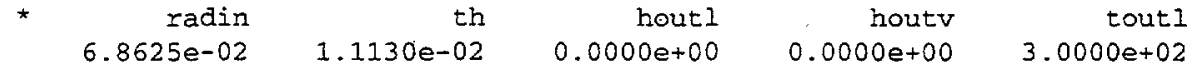

* toutv

$3.0000 e+02$

*

$* \mathrm{dx} * r 2 \quad 2.0000 e+00 \quad 1.0152 e+00 \quad r 41.2500 e+00 e$

* vol * r2 $2.9280 \mathrm{e}-02 \quad 1.4863 \mathrm{e}-02$ r4 $1.8300 \mathrm{e}-02 \mathrm{e}$

* fa *f $1.4640 \mathrm{e}-02 \mathrm{e}$

* fric * $1.6577 e-01$

* fric * $1.0361 \mathrm{e}-01$

$*$ elev * $-3.0000 e+00$

* hd *f $1.3653 \mathrm{e}-01 \mathrm{e}$

* nff * 1 r6 1 1e

*alp *f $0.0000 e+00 e$

*VI *f $6.771 e+00 e$

*vv *f $6.771 e+00 e$

*tI * $\mathrm{E} 3.4315 \mathrm{e}+02 \mathrm{e}$

$*$ tv $\quad$ f $3.4315 \mathrm{e}+02 \mathrm{e}$

$* \mathrm{p} * 5.16653 \mathrm{e}+05$

$* \mathrm{p} \quad * \quad 4.55935 \mathrm{e}+05$

* pa $\quad$ f $0.0000 e+00 e$

\section{$1.6577 e-01 \quad 2.5685 e-02$}

$1.0361 \mathrm{e}-01 \quad 1.0361 \mathrm{e}-01$

$0.1700 \mathrm{e}+00 \mathrm{~s}$

$-1.0000 e+00 \quad 5.0760 e-01$ r4 $1.0152 e+00 e$

$1 e$

$\begin{array}{lll}4.89 .762 e+05 & 4.71715 e+05 & 4.60768 e+05 s \\ 4.51102 e+05 & 4.46270 e+05 e\end{array}$

* $==============$ MODULE 5 (DOWNFLOW - PLENUM - UPFLOW) $===============$

* Water Pipe Connection After Water Supply Manifold in Loop13 


$\begin{array}{lrr}* * * * * * & \text { type } & \text { num } \\ \text { pipe } & & 513 \\ * & \text { ncells } & \text { nodes } \\ & 7 & 0 \\ * & \text { ichf } & \text { iconc } \\ * & 1 & 0 \\ * & \text { radin } & \text { th } \\ * & 7.6200 e-02 & 1.1130 e-02 \\ * & \text { toutv } & \end{array}$
ctitle
$513 \$ 513 \$$ Pipe conn.
junI
513
iacc
0
jun2
89

hout1

$0.0000 e+00$

epsw
$1.0000 e-05$

$* d x \quad * r 2 \quad 6.2500 e-01$

* vol * r2 $1.1401 \mathrm{e}-02$

* Ea. * E $1.8242 e-02 e$

* fric * $1.5000 e-01$

* fric * 1.0361e-01

* elev * r2 $1.7772 e+00$

* ha * E $1.5240 e-01 e$

*alp * $0.0000 e+00 e$

*vl *f $6.360 \mathrm{e}+00 \mathrm{e}$

* vv *f $6.360 e+00 e$

*t1 *E $3.2315 \mathrm{e}+02 \mathrm{e}$

* tv * $\ldots 3.2315 e+02 e$

$* \mathrm{p} * 7.11147 \mathrm{e}+05$

* p * 7.02841e+05

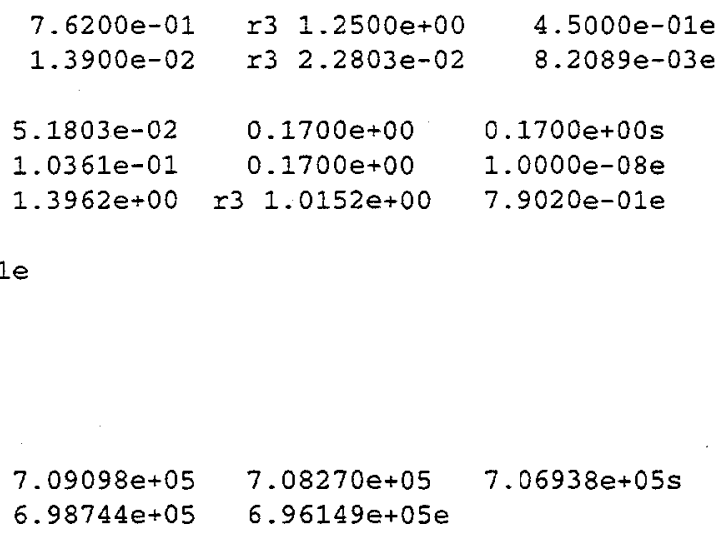

* Water Pipe Connection After Water Supply Manifold in Loop15 
$\star \mathrm{f} \quad 1.8242 e-02 e$

* fric * $1.0000 e-08$

* elev * $2.2500 e-01$

* ha * $\pm 1.5240 e-01 e$

* nff * $\quad-1 \quad r 21 \quad-1 e$

*alp * $0.0000 e+00 e$

$*$ vi $\star E 6.360 e+00 e$

$* v V * f 6.360 e+00 e$

$*$ tI $*$ f $3.2315 \mathrm{e}+02 \mathrm{e}$

$\star t v * f \quad 3.2315 e+02 e$

$\star \mathrm{p} * 7.00717 \mathrm{e}+05$

$\star$ pa $\quad$ f $0.0000 e+00 e$

$5.0000 e-02 \quad 5.0000 e-02$

$-1.0000 e+00-3.0000 e+00 e$

* Water Decoupler Upper Plenum in Loop13

* Decoupler Upper Plenum

$\begin{array}{lrrrr}* \star * * * * * & \text { type } & \text { num } & \text { id } & \text { ctitle } \\ \text { plenum } & & 510 & 510 & \$ 510 \$ \text { Dec UpperPI } \\ * & \text { npljn } & \text { iconc } & \text { juns } 1 & \text { juns } 2 \\ & 2 & 0 & 0 & 0\end{array}$

$\star$

* juncs * 510s *Inlet Ha to UPI

* juncs * 507e *UPI to Decoupler

$* \mathrm{dx} \quad * \mathrm{f} 2.0320 \mathrm{e}-01 \mathrm{e}$

* vol * $5.0000 e-01 e$

* elev * $\quad-4.0170 e+00 e$

*alp * $0.0000 \mathrm{e}+00 \mathrm{e}$

$* t I * 3.2315 \mathrm{e}+02 \mathrm{e}$

*tv * $3.2315 \mathrm{e}+02 \mathrm{e}$

* p * 7.33156e+05e

$* \mathrm{pa} * 0.0000 \mathrm{e}+00 \mathrm{e}$

*

*

* Module 5 Downflow

$\star \star \star \star \star \star \star x$ type num

pipe 507

* ncells nodes

* $\quad$ ichf $\quad$ iconc

* $\quad$ radin 1

$0.0000 e+00$

toutv1

$3.0000 e+02$

th1

$1.1130 \mathrm{e}-02$

$0.0000 e+00$

id

ctitle

$507 \$ 507 \$$ Decoupler water

jun1 jun2

507

iacc

0

hout 11
$00 \mathrm{e}+00$

503

ipow

houty

$0.0000 e+00$

tout 11

$3.0000 e+02$

*

$\star$

$\begin{array}{llllll}* & * & 0.3198 & \quad 4 & 0.6096 \quad 0.7418 \mathrm{e}\end{array}$

* vol * $\quad 1.7412 e-02$ r4 $3.3190 e-02$ 4.0388e-02e

* fa $* f \quad 5.4446 e-02 e$

* fric * $7.2950 e-02$ r5 $7.3700 e-02$

* elev * $-4.2785 e+00-4.7432 e+00$

* elev * $\quad-5.9624 \mathrm{e}+00-6.5720 \mathrm{e}+00$

* hd *f $3.1519 e-03 e$

* nff *f $1 e$

*alp *f $0.0000 e+00 e$

* VI *E $2.131 e+00 e$

* Vv * E 2.131e+00e

$* t 1 \quad * \mathrm{f} 3.2315 \mathrm{e}+02 \mathrm{e}$

$* \mathrm{tv} \quad * \mathrm{f} \quad 3.2315 \mathrm{e}+02 \mathrm{e}$

$* \mathrm{p} \quad$ * $7.26820 \mathrm{e}+05$

$7.19688 e+05 \quad 7.10405 e+05$

$7.01151 \mathrm{e}+05 \mathrm{~s}$ 
$\begin{array}{lll}* \mathrm{p} \quad * \quad 6.91915 e+0 \\ * \mathrm{pa} \quad * \mathrm{f} & 0.0000 e+00\end{array}$

*

* Module 5 Mid-plenum

$* * * * * * *$ type num

plenum 503

* npljn $\quad$ iconc

$6.81704 e+05 e$

* juncs * 503s *Dec to Down P1

* juncs * 504e *Down Pl to Rowl

$* \mathrm{dx} * \mathrm{f} \quad 7.6200 \mathrm{e}-02 \mathrm{e}$

* vol * 5.0000e-01e

$\star$ elev * $\quad-7.6567 e+00 e$

$*$ alp * $0.0000 e+00 e$

*t1 * $3.2515 \mathrm{e}+02 \mathrm{e}$

*tv * $\quad 3.2515 \mathrm{e}+02 \mathrm{e}$

* $\mathrm{p} \quad$ * $6.76187 \mathrm{e}+05 \mathrm{e}$

$*$ pa $* 0.0000 e+00 e$

*

*

* Module 5 upflow

$* \star * \star * * *$ type

pipe

num

500

* - ncells

* ncells

* ichf

* radín

$0.0000 e+00$

toutv

$3.0000 e+02$

*

* dx

* vol

*

$0.7418 \quad r 4$

0.6096

id ctitle

$500 \$ 500 \$$ Rowl water

* fa * f $5.4446 \mathrm{e}-02 \mathrm{e}$

* fric * $7.5000 e-01$

* elev * $\quad-7.2477 e+00$

* elev * $\quad-5.3528 e+00$

* ha *f $3.1519 e-03 e$

* nff * $f$ le

*alp * $\mathrm{f} \quad 0.0000 \mathrm{e}+00 \mathrm{e}$

* vi * f $2.137 e+00 e$

* vv * E $2.137 e+00 e$

*tI *

*tv *f $3.2815 e+02 e$

* $\mathrm{p}$ * $6.58253 \mathrm{e}+05$

* $\mathrm{p} * 5.72203 e+05$

* pa * E $0.0000 e+00 e$

* Water Upper Plenum in Loop13

* Upper Rowl Plenum

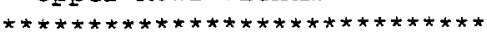

$\star * * * * *$ type

plenum

508

* npljn iconc

2
3. $3190 e-02$

$0.3198 \mathrm{e}$

r5 $7.3700 \mathrm{e}-02 \quad 7.2950 \mathrm{e}-01 \mathrm{e}$

$-6.5720 e+00 \quad-5.9624 e+00 s$

$-4.7432 e+00 \quad-4.2785 e+00 e$

\section{$6.34994 e+05$ \\ $6.14038 e+05$ \\ $5.93114 e+05 s$}

$5.56226 e+05 e$

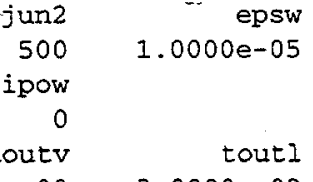

$3.0000 e+02$

* 
* juncs * 500s *RI to Upper PI

* juncs * 508e *Upper Pl to Oulet Header

*dx *f $2.0320 e-01 e$

* vol * 5.0000e-01e

* elev * $-4.0170 e+00 \mathrm{e}$

* alp * $0.0000 \mathrm{e}+00 \mathrm{e}$

* tI * $3.3015 \mathrm{e}+02 \mathrm{e}$

* tv * $3.3015 e+02 e$

* p * 5.47939e+05e

* pa * $\quad 0.0000 \mathrm{e}+00 \mathrm{e}$

* Water Pipe Before Water Return Manifold in Loop13

$* * * * * * *$ type num

pipe

ncells

511

7

ichf

nodes

id

ctitle radin

$7.6200 \mathrm{e}-02$

* toutv

$1.1130 \mathrm{e}-02$

icone

0

th

$3.0000 e+02$

* dx * r2 $2.0000 e+00$

* vol

* $r 2$

$3.6484 \mathrm{e}-02$

$1.0152 \mathrm{e}+00$

r4 $1.2500 \mathrm{e}+00 \mathrm{e}$

* fa

$\star \pm$$$
1.8242 \mathrm{e}-02 \mathrm{e}
$$

* Eric * $1.6577 \mathrm{e}-01$

* fric

* elev

* hd

* 1.0361e-01

* $\quad-3.0000 e+00$

* $1.5240 e-01 e$

* nff

* alp$$
\text { * } 1 \text { r6 } 1
$$

* $0.0000 e+00 e$

vl * $\mathrm{vl} 6.381 \mathrm{e}+00 \mathrm{e}$

*vv * v $6.381 e+00 e$

$* t 1 *$ E $3.3015 e+02 e$

*tv *f $3.3015 \mathrm{e}+02 \mathrm{e}$

* $\mathrm{p} * 5.12934 \mathrm{e}+05$

* $\mathrm{p} \quad$ * $4.55284 e+05$

$*$ pa $\quad$ f. $0.0000 e+00 e$
$4.87045 e+05$
$4.69522 \mathrm{e}+05$
$4.59378 e+05 s$
$4.51189 e+05$
$4.47095 e+05 e$

$0.1700 \mathrm{e}+00 \mathrm{~s}$
$1.0361 \mathrm{e}-01$
$5685 e-02$
$0.5000 e+00 e$

*

*

$\star$

* Water Pipe Connection After Water Supply Manifold in Loop15

$\star \star \star \star \star \star *$ type num id ctitle

pipe

541

* ncells nodes 7

541 \$541\$ Pipe conn. jun1

541
iacc

1

th

1. 1130 e-02

$0.0000 e+00$

0
hout I
oet00

0.0000 houtv

jun2

83

ipow

1.0000 sw

tout 1

$3.0000 e+02$

4.9213e-02

toutv

$3.0000 e+02$

*

$\star \mathrm{dx} \quad$ * r2 $6.2500 e-01$

* vol * r2 $3.8973 e-02$

* fa * f $6.2356 e-02 e$

* fric * $1.5000 e-01$

* fric * $5.0000 e-02$

$7.6200 e-01$

$4.7515 e^{-02}$

$5.0000 e-02$

$5.0000 e^{-02}$
$131.2500 e+00$

r3 $7.7945 e-02$

$0.1700 e+00$

$0.1700 e+00$
$4.5000 e-01 e$

$2.8060 e-02 e$

$0.1700 \mathrm{e}+00 \mathrm{~s}$

$1.0000 e-08 e$

toutz

$3.0000 e+02$

psw

$5111.0000 e-05$

1 


$$
\text { * }
$$

* elev * $1.7772 e+00$

$1.7772 e+00$

1. $3962 e+00$

$1.0152 \mathrm{e}+00 \mathrm{~s}$

* elev *

$1.0152 e+00$

1. $0152 \mathrm{e}+00$

$7.9020 e-01 e$

* ha

* $9.8425 e-02$

* nff

* alp$$
\text { * } 1
$$

$1 \pm 6 \quad 1$

$-1 e$

* vI

* f $0.0000 e+00 e$

*f $6.569 \mathrm{e}+00 \mathrm{e}$

* vv *f $6.569 \mathrm{e}+00 \mathrm{e}$

*tI *f $3.2315 \mathrm{e}+02 \mathrm{e}$

*tv *f $3.2315 \mathrm{e}+02 \mathrm{e}$

* p * $7.08881 e+05$

* $p$ * $\quad$ * $6.96526 e+05$

* pa * f $0.0000 e+00 e$

$7.06003 e+05$

$6.91838 e+05$

$7.04061 e+05$

$6.87929 e+05 e$

ctitle $\$ 83 \$$ Dec UpperPI juns2

* juncs * $83 \mathrm{~s}$ *Inlet Ha to UPl

* juncs * 84 e *UPl to Decoupler

* dx * f $1.1520 e-01 e$

* vol * $7.1834 \mathrm{e}-03 \mathrm{e}$

* elev * 5.0760 e-01e

*alp * $0.0000 e+00 e$

* ti * $\quad$ * $3.2315 \mathrm{e}+02 \mathrm{e}$

* tv * $3.2315 e+02 e$

* $\mathrm{p} * 7.11192 \mathrm{e}+05 \mathrm{e}$

*.pa * $\quad$. $0.0000 e+00 e$

* Water Pipe Connection After Water Supply Manifold in Loop15 $* * \star * \star * *$ type pipe

ncells

* ich

* radín

4.9213e-02 toutv

$3.0000 e+02$

* dx * $\quad$ * $4.5000 e-01$

* vol * $2.8060 e-02$

* fa $*$ f $6.2356 e-02 e$

* fric * 1.0000 e-08

* Eric * 5.0000e-02

* Eric * 1.0000 e-08e

* elev * 2.2500 e-01

* elev * $-3.4765 e+00$

* ha * f $9.8425 e-02 e$

*nff * $\quad-1 \quad r 71 \quad-1 e$

*alp *f $0.0000 e+00 e$

$* v 1 * \mathrm{f} 6.569 e+00 \mathrm{e}$

*v * $\mathrm{v} \quad 6.569 \mathrm{e}+00 \mathrm{e}$

*tl *f $3.2315 \mathrm{e}+02 \mathrm{e}$

*tV * f $3.2315 \mathrm{e}+02 \mathrm{e}$

* $\mathrm{p} \quad$ * $6.91775 \mathrm{e}+05$

* $\mathrm{p} \quad 7.12690 \mathrm{e}+05$

r7 9.9330e-01e

r7 6.1938e-02e

$$
5.0000 e-02
$$

$5.0000 e-02$

$5.0000 e-02$

$5.0000 e-02 s$

$-4.9665 e-01$

$5.0000 e-02$

$5.0000 e-02 s$

$-4.4698 \mathrm{e}+00$

$-1.4899 e+00$

$-2.4832 e+00 s$

$-5.4631 e+00$

$-6.4564 e+00 e$

(n)

$$
\begin{aligned}
& 6.95618 e+05 \\
& 7.12690 e+05
\end{aligned}
$$

$7.01308 e+05$

$7.06999 \mathrm{e}+05 \mathrm{~s}$

$7.24073 e+05 \quad 7.29764 e+05 e$ 
$\star$

*

* Water Decoupler Upper Plenum in Loop15

* Decoupler Upper Plenum

$\begin{array}{lrrrr}* * * * * * & \text { num } & \text { id } & \text { ctitle } \\ \text { plenum } & \text { type } & 538 & 538 & \$ 538 \$ \text { Dec Upperpl } \\ \text { * } & \text { npljn } & \text { iconc } & \text { junsl } & \text { juns } 2 \\ & 2 & 0 & 0 & 0\end{array}$

* juncs * $538 \mathrm{~s}$ *Inlet Hd to UPl

* juncs * 535 e *UPI to Decoupler

*

* dx *f $8.5060 e-02 e$

* vol * 5.0000e-01e

* elev * $\quad-6.9956 e+00 e$

* alp * $0.0000 e+00 e$

* $t 1$ * $3.2315 e+02 e$

* tv * $3.2315 e+02 e$

* $\mathrm{p} \quad$ * $7.33883 \mathrm{e}+05 \mathrm{e}$

* pa * $0.0000 e+00 e$

$\star$

* Decoupler Water in Loop15

* Water in Decoupler

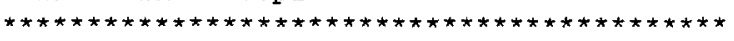

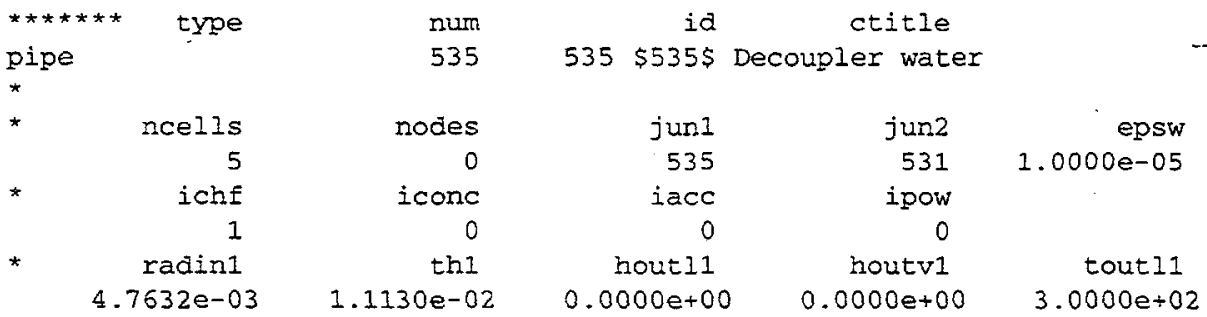

toutv1

$3.0000 e+02$

$\star$

* dx * 4.9580e-01

* vol * $\quad 7.9412 e-02$

* fa * f $1.6017 e-01 e$

* fric * $7.8128 e-01$

* elev * $\quad-7.0381 e+00$

* elev * $-7.0381 e+00$

* hd *f $9.5264 e-03 e$

* nff * $1 \quad r 4-1$

*alp * f $0.0000 e+00 e$

* v1 * $\quad$ \& $2.557 \mathrm{e}+00 \mathrm{e}$

* vv * $\quad 2.557 \mathrm{e}+00 \mathrm{e}$

*tI * $\mathrm{E} \quad 3.2315 e+02 e$

*tV * E $3.2315 e+02 e$

$\star \mathrm{p}$ * $7.26093 e+05$

* $\mathrm{p}$ * $7.04759 \mathrm{e}+05 \mathrm{e}$

* pa * p $0.0000 e+00 e$

$r 4.9580$ e-01e

$r 47.9412 e-02 e$

$\Upsilon 43.6096 e-01$

$-7.0381 e+00$

$4.9804 e-01 e$

$-7.0381 e+00 e$

$-7.0381 \mathrm{e}+00 \mathrm{~s}$

$1 e$

* Lower Water Plenum in Loop15

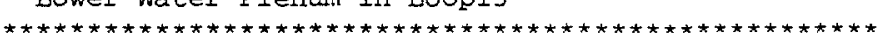

* Plenum for Lumped Single Loop Blanket System

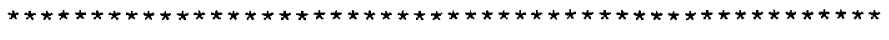

$\star * * * * *$ type num id ctitle 


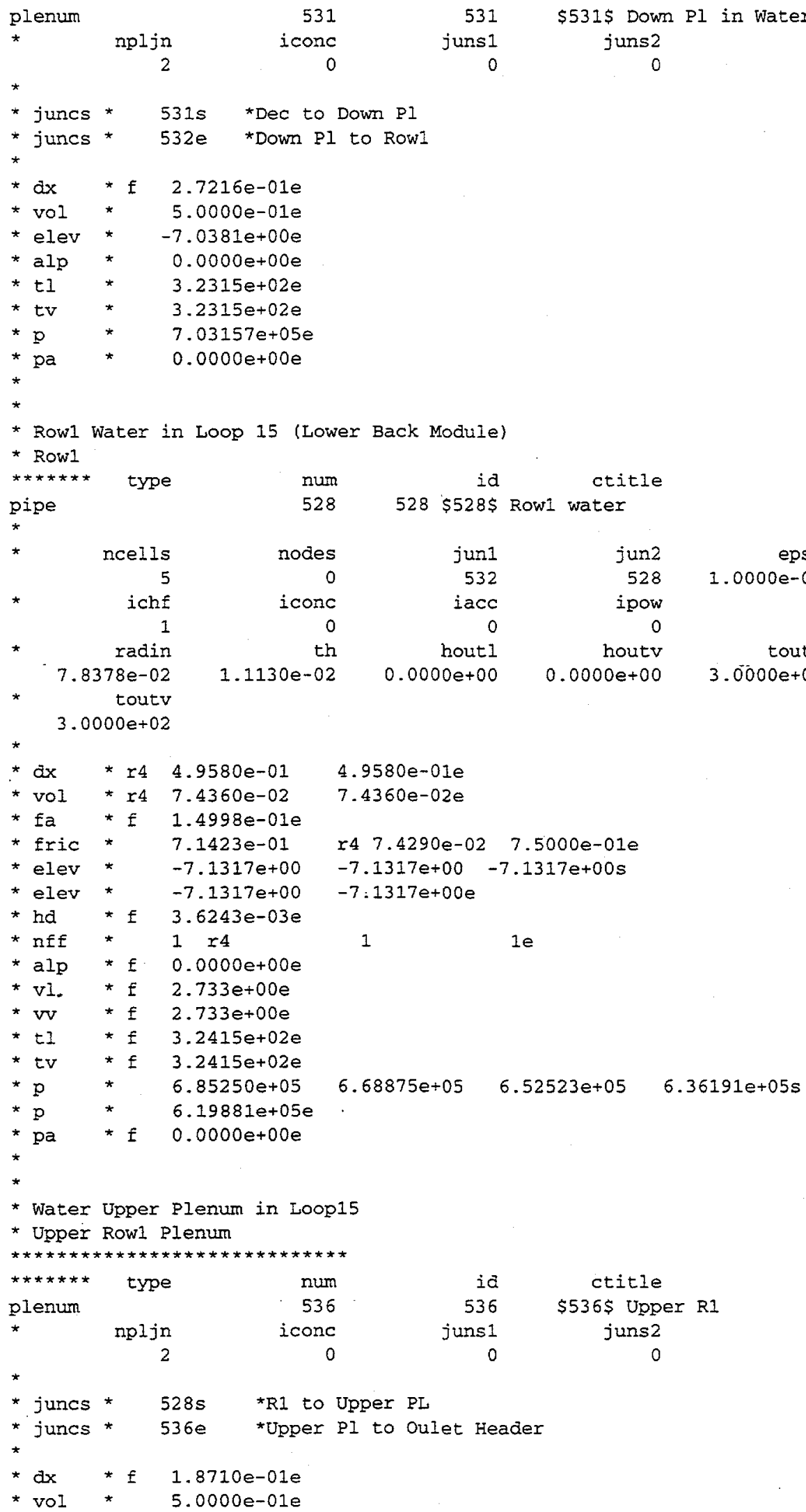

plenum

nplin

531

icone

0

*

* juncs * $531 \mathrm{~s}$ *Dec to Down PI

* juncs * 532e *Down Pl to Rowl

* dx *f $2.7216 \mathrm{e}-01 \mathrm{e}$

* vol * 5.0000e-01e

* elev * $\quad-7.0381 e+00 e$

*alp * $0.0000 e+00 e$

$*$ t1 * $3.2315 \mathrm{e}+02 \mathrm{e}$

* tv * $3.2315 e+02 e$

* $\mathrm{p}$ * 7.03157e+05e

$*$ pa * $\quad 0.0000 e+00 e$

$*$

* Rowl water in Loop 15 (Lower Back Module)

* Row1

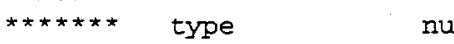

pipe 528

$528 \$ 528 \$$ Rowl water

* ncelis

* ichE

iche

radin

$7.8378 e-02$

toutv

$3.0000 e+02$

*

*dx * r4 4.9580e-01

* vol * r4 7.4360e-02

* fa *f $1.4998 \mathrm{e}-01 \mathrm{e}$

* fric * 7.1423e-01

* elev * $-7.1317 \mathrm{e}+00$

* elev * $-7.1317 e+00$

* hd *f $3.6243 e-03 e$

* nff * 1 r4

$*$ alp *f $0.0000 e+00 e$

*vl. *f $2.733 e+00 e$

*vv *f $2.733 e+00 e$

$\star t I \quad * \pm 3.2415 e+02 e$

*tv * $t$. $3.2415 e+02 e$

$* p \quad * \quad 6.85250 e+05$

$* \mathrm{p} * 6.19881 \mathrm{e}+05 \mathrm{e}$

* pa *f $0.0000 e+00 e$

$\star$

*

* Water Upper Plenum in Loop15

* Upper Row1 Plenum

$\begin{array}{lrrrc}\star \star * \star * * * & \text { type } & \text { num } & \text { id } & \text { ctitle } \\ \text { plenum } & & 536 & 536 & \$ 536 \$ \text { Upper R1 } \\ \star & \text { npljn } & \text { iconc } & \text { juns1 } & \text { juns2 } \\ & 2 & 0 & 0 & 0\end{array}$

* juncs * 528s *RI to Upper PL

* juncs * 536e *Upper PI to Oulet Header

$\star \mathrm{dx} * \mathrm{f} 1.8710 \mathrm{e}-01 \mathrm{e}$

* vol * $5.0000 \mathrm{e}-01 \mathrm{e}$

$4.9580 \mathrm{e}-01 \mathrm{e}$

$7.4360 \mathrm{e}-02 \mathrm{e}$

r4 $7.4290 \mathrm{e}-02 \quad 7.5000 \mathrm{e}-01 \mathrm{e}$

$-7.1317 e+00-7.1317 e+00 \mathrm{~s}$

$-7: 1317 e+00 e$

$1 \quad 1 e$

$6.68875 e+05 \quad 6.52523 e+05 \quad 6.36191 e+05 s$

63 of 96 
$*$ elev * $\quad-7.1317 e+00 e$

$*$ alp * $0.0000 e+00 e$

* t * $* 3.2615 e+02 e$

* tv * $3.2615 \mathrm{e}+02 \mathrm{e}$

* p * $\quad$ * $6.09765 e+05 e$

* pa * $\quad$ pa $0000 \mathrm{e}+00 \mathrm{e}$

*

*

* Water Pipe Before Water Return Manifold in Loop15

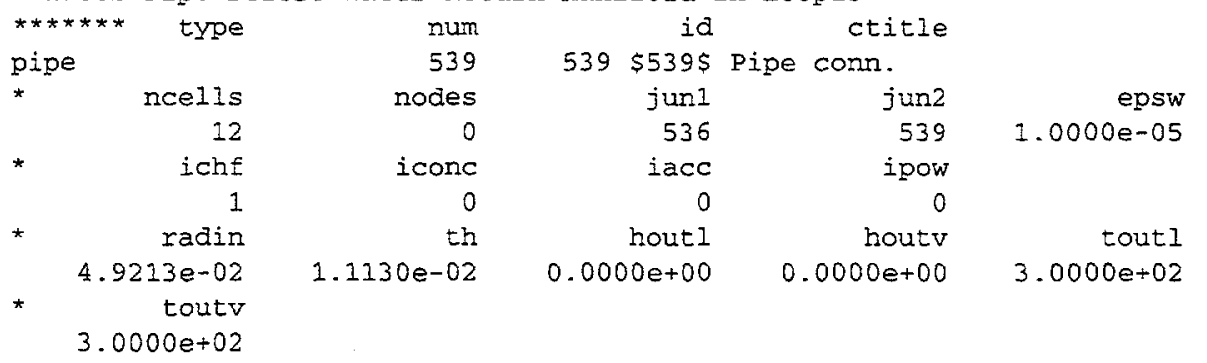

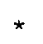

$* d x \quad * \quad r 7 \quad 1.0055 e+00$

$1.0152 e+00 \quad r 41.2500 e+0.0 e$

* vol *r7 $6.2599 \mathrm{e}-02$

* fa *E $6.2356 \mathrm{e}-02 \mathrm{e}$

* fric * $5.0000 e-02$

* fric * 5.0000e-02

* fric * $0.1700 e+00$

* Exic * $0.5000 e+00 e$

* elev * $\quad-6.5354 e+00$

* elev * $-2.5136 e+00$

* elev * r4 $1.0152 e+00 e$

* hd * $\pm 9.8425 e-02 e$

*nff * $\quad-1 \quad 2111$

*aip * $\quad 0.0000 e+00 e$

*vl *f $6.580 e+00 e$

$* v v * E \quad 6.580 e+00 e$

$* t 1 *$ E $3.2615 e+02 e$

*tV *E $3.2615 e+02 e$

$* \mathrm{p} * \quad 5.69483 e+05$

* $\mathrm{p} \quad$ * $5.14634 \mathrm{e}+05$

* $\mathrm{p} * 4.61615 \mathrm{e}+05$

* pa *f. 0.0000 e $+00 e$
$5.55771 e+05$
$5.00923 e+05$
$5.42059 e+05$
$5.28347 e+05 s$
$4.56933 e+05$
$4.87211 e+05$
$4.73438 \mathrm{e}+05 \mathrm{~s}$
$4.52252 e+05$
$4.47571 e+05 e$

*

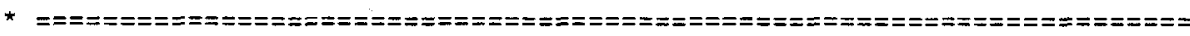

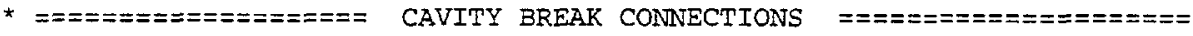

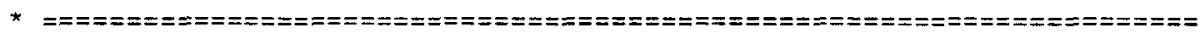

*

$*$ .

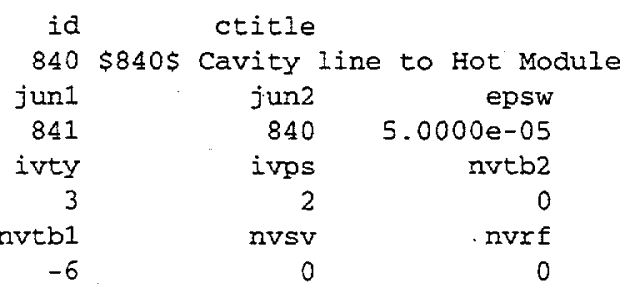
valve

type

* ncells

2

* ichf

* $\quad$ ivtr

108

* ivtrov

$\operatorname{rvm} x$

$1.0000 e+01$

radin

$0.0000 e-00$

$8.1800 e-03$
$+00$

num
840

nodes

icone

ivsv

8

ivtyov

0

00 fminov

$0.0000 e+00$

hout 1

$0.0000 e+00$ fmaxov

$1.0000 e+00$

houtv

$0.0000 e+00$ tout 1

$3.3300 e+00$ 


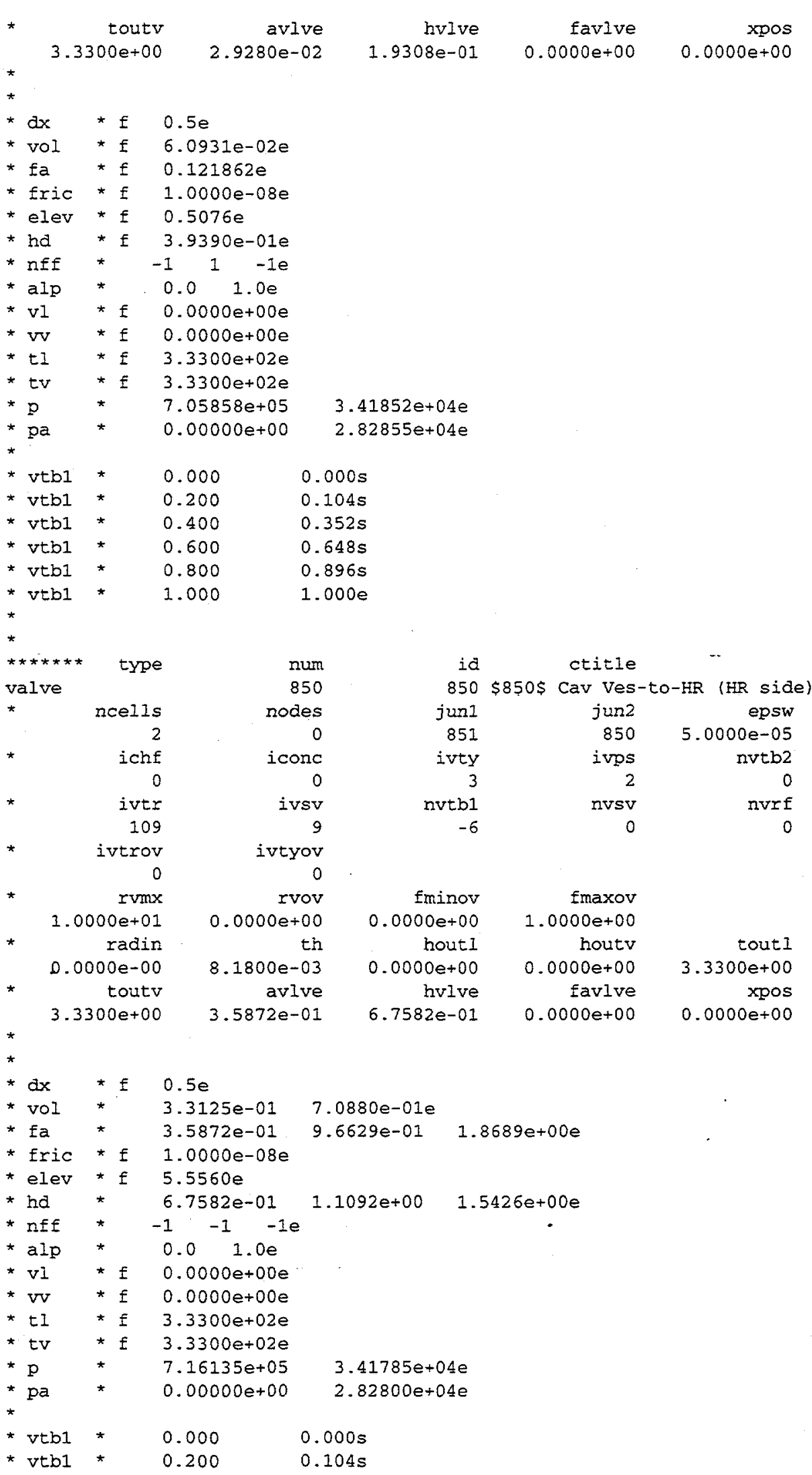


Report:

Section:

Date:

TRAC BLANKET SYSTEM MODEL

Page:
WSRC-TR-98-0053

Appendix A

$07 / 14 / 98$

66 of 96

* vtb1 * 0.600

$0.352 \mathrm{~s}$

$0.648 \mathrm{~s}$

* vebi

1.000

$0.896 \mathrm{~s}$

* vtbl

$1.000 e$

$\begin{array}{lr}* * * * * * * & \text { type } \\ \text { valve } & \\ * & \text { ncelis } \\ * & 2 \\ & \text { ichf } \\ * & 0 \\ * & \text { ivtr } \\ & 109 \\ * & \text { ivtrov } \\ & \text { rvmx }\end{array}$

num

852

nodes

icone

0

9

ivtyov

$$
0
$$

rvov

$0.0000 e+00$

radin

$0.0000 \mathrm{e}-00$

toutv

$3.3300 e+00$
$8.1800 e-03$

avive

$3.5872 e-01$

$\begin{array}{rrr}\text { id } & \text { ctitle } & \\ 852 & \$ 852 \$ \text { Cav ves-to-HR } & \text { (Header side) } \\ \text { jun1 } & \text { jun2 } & \text { epsw } \\ 853 & 852 & 5.0000 \text { e- } 05 \\ \text { ivty } & \text { ivps } & \text { nvtb2 } \\ 3 & 2 & 0 \\ \text { nvtb1 } & \text { nvsv } & \text { nvrf } \\ -6 & 0 & 0\end{array}$

fminov

$0.0000 e+00$

houtl

$0.0000 e+00$

hvive

$6.7582 e-01 \quad 0.0000 e+00$
$1.0000 e+00$

houty

$0.0000 e+00$

favlve

$0.0000 e+00$ tout I

$3.3300 e+00$

0.0000

*

*dx $\quad$ f $\quad 0.5 \mathrm{e}$

* vol * $3.3125 \mathrm{e}-01$

* fa * $3.5872 \mathrm{e}-01$

* Eric * f $1.0000 e-08 e$

* elev * f $5.5560 \mathrm{e}$

* hd * $\quad 6.7582 \mathrm{e}-01 \quad 1.1092 \mathrm{e}+00 \quad 1.5426 \mathrm{e}+00 \mathrm{e}$

*nff * $\quad-1 \quad-1 \quad-1 e$

*aip * $0.01 .0 \mathrm{e}$

* vl $*$ f $0.0000 e+00 e$

* vv *f $0.0000 e+00 e$

$* t z * \pm 3.3300 e+02 e$

*tv *f $3.3300 e+02 e$

$* p \quad * \quad 7.16135 e+05$

$\star$ pa $* 0.00000 e+00$

$3.41785 e+04 e$

$2.82800 e+04 e$

* vtb1 *

0.000

$7.0880 e-01 e$

$9.6629 \mathrm{e}-01$

$1.8689 e+00 e$

* vtbI* $* 0.200$

$0.000 \mathrm{~s}$

* vtbI*

* vtb1 *

0.400

$0.104 \mathrm{~s}$

0.600

$0.352 \mathrm{~s}$

* vtb1*

* vtbI *

0.800

$0.648 \mathrm{~s}$

*

*

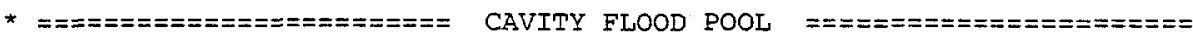

*

*

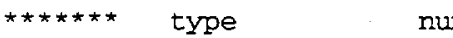

break 801

* jun 1 ibty

810

ibty

dxin

volin

$1.0000 e+00$

pain

$1.3936 e+02$

concin

$0.896 \mathrm{~s}$

$1.000 \mathrm{e}$

$9.3946 e+04$

$0.0000 e+00$

alpin
$1.0000 e+00$
rbmx
$1.0000 e+02$

id ctitle

$801 \$ 801 \$$ bk BC on cavity pool isat ioff

lpin

0

tin

pin

$3.1315 e+02$

$1.0133 e+05$

$0.0000 e+00$

$8.0736 e+00$ 


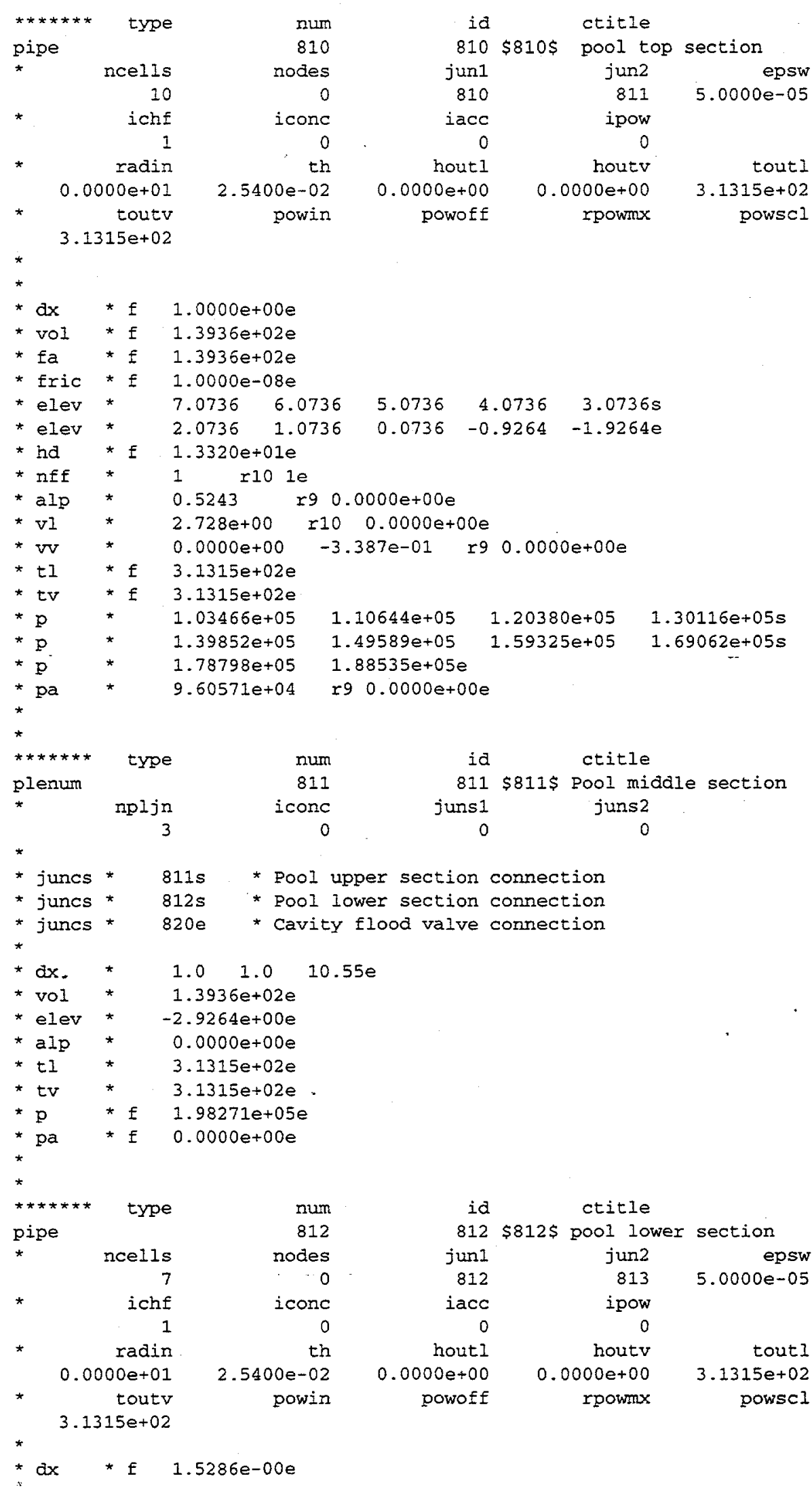


Report:

Section:

Date:

Page:
WSRC-TR-98-0053

Appendix A $07 / 14 / 98$ 68 of 96

TRAC BLANKET SYSTEM MODEL

* $\quad 2.1303 e+02 e$

* fa * E $1.3936 \mathrm{e}+02 \mathrm{e}$

* fric * $0.0000 e+00 e$

* elev * $\quad-4.1907 \quad-5.7193 \quad-7.2479 \quad-8.7765 \mathrm{~s}$

* elev * $\quad-10.3051-11.8337 \quad-13.3623 \mathrm{e}$

* ha *f $1.3320 e+01 e$

*nff *f $1 e$

$*$ alp *f $0.0000 e+00 e$

*v1 * e $0.0000 e+00 e$

* vv *f $0.0000 e+00 e$

$* t I * \pm 3.1315 e+02 e$

*tv * $\quad$ t.1315e+02e

$* p \quad * \quad 2.10581 e+05$

*p * $\quad$ * $2.70116 e+05$

$* \mathrm{pa} \quad *$ f $0.0000 e+00 e$

$2.25465 \mathrm{e}+05 \quad 2.40348 \mathrm{e}+05 \quad 2.55232 \mathrm{e}+05 \mathrm{~s}$

$2.84999 e+05 \quad 2.99883 e+05 e$

plenum.

type

num

* npljn

813

pljn

icone

0

id

ctitle

$813 \$ 813 \$$ pool bottom

*

* juncs * $813 \mathrm{e} \quad$ *Connected to pool 4

* dx * 1.5311e-01e

* vol * 2.1338e+01e

$*$ elev * $-1.4203 e+01 e$

*alp * $\quad 0.0000 e+00 e$

$* t 1 * 3.1315 \mathrm{e}+02 \mathrm{e}$

$* t v * 3.1315 \mathrm{e}+02 \mathrm{e}$

$* \mathrm{p} \quad * \quad 3.08069 \mathrm{e}+05 \mathrm{e}$

* pa * $\quad$ a $0000 e+00 e$

$\star$

*

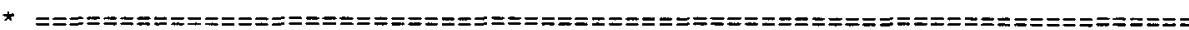

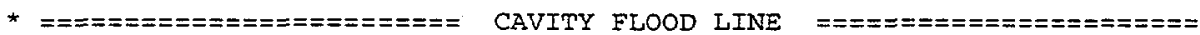

*

*

$* * * * * * *$ type num

pipe $\quad 820$

* nceils nodes

13 icone

* ichf

radin

$0.0000 e-01$

toutv

$3.1315 e+02$
1. 0000 e- 02

powis

0

juns2

*

*dx * $\quad 2 \quad 1.25 \quad r 61.0 \quad r 31.8334 \quad r 21.053 e$

* vol * r2 $9.0134 \mathrm{e}-02$ r6 $7.2107 \mathrm{e}-02 \quad r 31.322 \mathrm{e}-01 \mathrm{~s}$

* vol * r2 $7.5929 e-02 e$

* fa *f $7.2107 e-02 e$

* fric * r8 1.0000e-08 0.172 r2 1.0000e-08s

* fric * $0.172 \quad r 21.0000 \mathrm{e}-08 \mathrm{e}$

* elev * r2 $-2.9264 \quad-3.0387 \quad-3.2632 \quad-3.4877 \quad-3.7122 \mathrm{~s}$

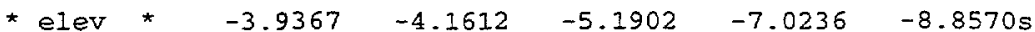

* elev * r2 $-9.7737 \mathrm{e}$

* hd * E $3.0300 \mathrm{e}-01 \mathrm{e}$

* nff * $-1 \quad$ ri3 $1 e$ 
*alp *f $0.0000 e+00 e$

*vl * E $0.0000 e+00 e$

*vV $* \mathrm{f} \quad 0.0000 e+00 e$

$* t I * f \quad 3.1315 e+02 e$

*tV * E $3.1315 e+02 e$

* pn * $\quad 1.98262 \mathrm{e}+05$

* pn * $2.03678 \mathrm{e}+05$

$\star$

$1.98262 e+05$

$1.99345 \mathrm{e}+05$

$2.05844 e+05$

$2.08010 e+05$

$2.01511 e+05 s$

* pn

$2.20104 e+05$

$2.37793 e+05$

$2.55482 e+05$

$2.10176 \mathrm{e}+05 \mathrm{~s}$

* pan

× $0.00000 e+04 e$

$\star \star \star \star \star \star * \star$ type num

valve 821

* ncells nodes

ichf icon

iconc

ivst

ivtr

102

12

ivtrov

0

ivtyov

rvmx

$1.0000 e+01$

radin

$0.0000 e-00$

toutv

$3.1315 e+02$

rvov

th

$1.0000 e-02$

avlve

$7.2107 e-02$
frinov
$0.0000 e+00$
hout 1
$0.0000 e+00$
hvlve
$3.0300 \mathrm{e}-01$

id ctitle

821 \$821\$ Cavity flood Iine valve

jun1 jun2

821

ivty

3

nvtbl

$-6$

822

$5.0000 e-05$

nvtb2

2

nvsv

0

$+$

* dx * $\quad 1.5 \quad 1.0 \mathrm{e}$

* vol * $1.0816 e-01$

$\star$

E

$7.2107 e-02 e$

* Eric

$1.0000 \mathrm{e}-08$

$7.2107 e-02 e$

* elev *f $-9.7737 e+00 e$

* ha *f $3.0300 e-01 e$

* nff * E $1 e$

*alp * $\quad$ a $0.0000 e+00$

*VI * E $0.0000 e+00 e$

* vv *E $0.0000 e+00 e$

*t. * $\quad 3.1315 e+02$

*tv $* 3.1315 e+02$

$* \mathrm{p} \quad * \quad 2.64327 \mathrm{e}+05$

* pa * $\quad 0.0000$ e+00

0.088

$0.172 \mathrm{e}$

* vtb1 * 0.00 .000 s

* vtb1 * $0.20 .104 \mathrm{~s}$

* vtb1 * $0.40 .352 \mathrm{~s}$

* vtb1 * $0.60 .648 \mathrm{~s}$

* vtb1 * $0.80 .896 \mathrm{~s}$

* vtb1 * $1.01 .000 \mathrm{e}$

*

$\star \star \star \star \star * *$ type num

pipe

* ncells nodes

1. $0000 \mathrm{e}+00 \mathrm{e}$

$3.3300 \mathrm{e}+02 \mathrm{e}$

$3.3300 \mathrm{e}+02 \mathrm{e}$

$3.42228 \mathrm{e}+04 \mathrm{e}$

2. $83164 e+04 e$

* 1 o

ichf icone

radin

$0.0000 e-01$

toutv

1. 0000 e- 02

powin

\begin{tabular}{|c|c|c|c|}
\hline$i d$ & & ctitle & \\
\hline 822 & $\$ 822 \$$ & Flood line & internal \\
\hline junl & & jun2 & epsw \\
\hline 822 & & 823 & $5.0000 e-05$ \\
\hline iacc & & ipow & \\
\hline 0 & & 0 & \\
\hline houtl & & houty & toutl \\
\hline $0.0000 e+00$ & 0.0 & $0000 e+00$ & $3.3300 e+02$ \\
\hline powoff & & rpowmx & powscl \\
\hline
\end{tabular}

1. 0000 e+00

$000 e+00$

0.0000 e+00

tout 1

3. $1315 e+02$

xpos

$0.0000 e+00$ 


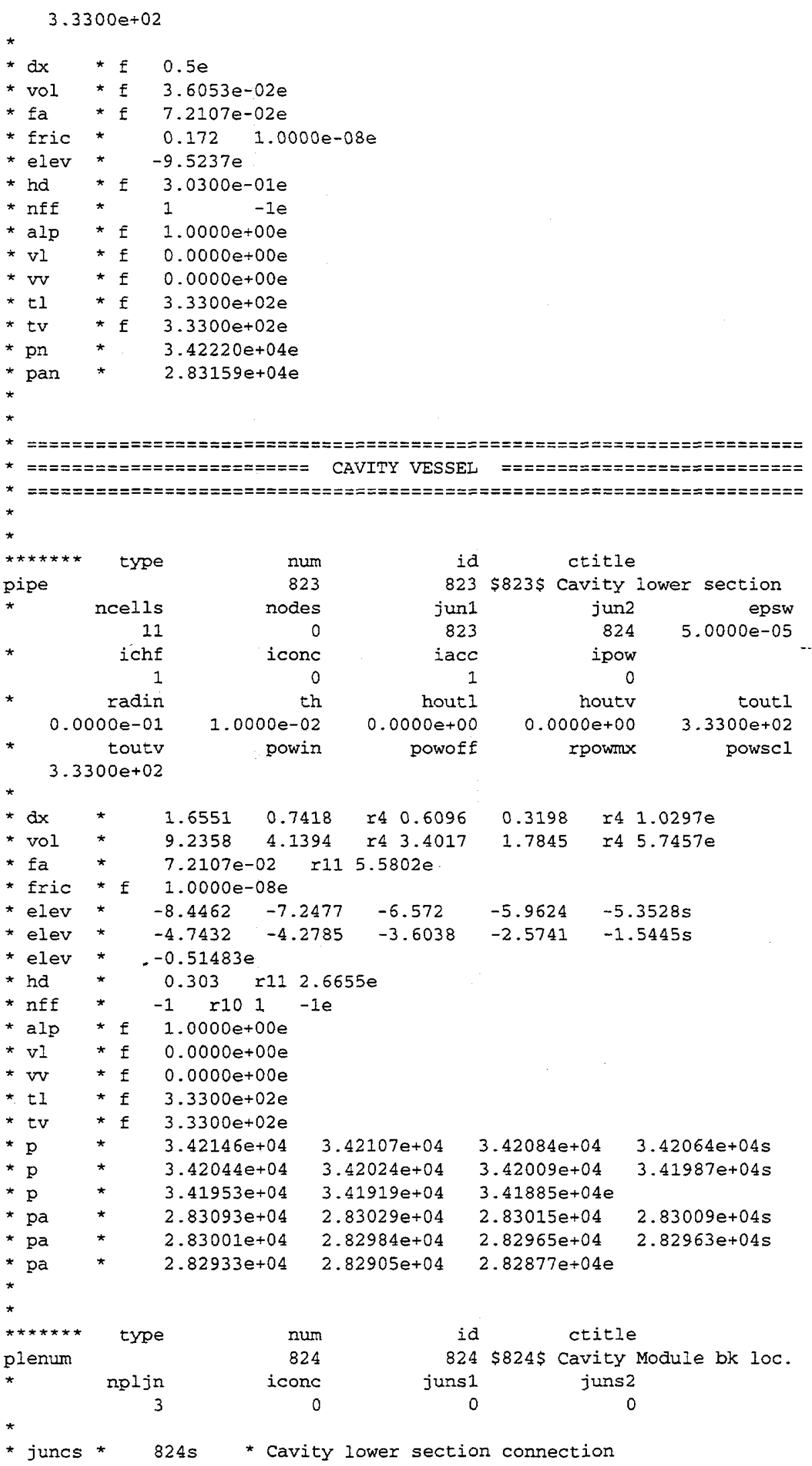


* juncs * $825 \mathrm{~s}$ * Cavity midale section connection

* juncs * $840 \mathrm{e}$ * Cavity line connection to Hot Module

* dx * r2 $1.0152 \quad 9.0964 \mathrm{e}$

* vol * $1.0696 \mathrm{e}+02 \mathrm{e}$

* elev * $0.5076 \mathrm{e}$

*alp * $\quad 1.0000 e+00 e$

$* t 1 * 3.3300 e+02 e$

*tv * $\quad$ tv $3.3300 e+02 e$

* p *f $3.41852 e+04 e$

* pa $* f \quad 2.82855 e+04 \mathrm{e}$

*

*

$\star \star \star \star \star \star *$ type num

id id ctitie

Page:

71 of 96

pipe 825

$\star$ ncells nodes

$\begin{array}{rr}4 & 0 \\ * \quad \text { ichf } & \text { iconc }\end{array}$

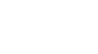

radin

$0.0000 e-01$

toutv

1.0000 e- 02

powin

$3.3300 e+02$

th

$0.0000 e+00$

powofE
$825 \$ 825 \$$ Cavity middle section
jun1
jun2
826

825

iacc

1

$\begin{array}{lllll}* \mathrm{dx} * & 1.0168 & \text { r3 } & 1.0267 \mathrm{e}\end{array}$

* fa *f $1.0536 e+02 e$

* Eric *f $1.0000 e-08 e$

* elev *

* ha

* nff

* alp

* v1

$* v \mathrm{v}$

$* t 1$

* tv

* p

* pa

*

*

****** type nu

plenum

*

type

1.5236

2.5454

3.5721

$4.5988 e$

f $11.582 \mathrm{e}$

* $\mathrm{f} 1 \mathrm{e}$

* f $1.0000 e+00 e$

* E $0.0000 e+00 e$

* E $0.0000 e+00 \mathrm{e}$

* f $3.3300 \mathrm{e}+02 \mathrm{e}$

* $\quad 3.3300 e+02 \mathrm{e}$

* f $3.41818 \mathrm{e}+04 \mathrm{e}$

* $2.82822 e+04 e$

$\star$

* juncs * $826 s$

* juncs * $828 \mathrm{~s}$

* juncs * $850 \mathrm{~s}$

* juncs * $852 \mathrm{e}$

num
826
icone
0

id

ctitle

$826 \$ 826 \$$ Cavity HR bk loc. juns1 juns2 ipow

houtv

rpowmix

$3.3300 e+02$
powscl

$\star$

* $d x \quad$ * r2 $0.888 \quad r 29.0964 \mathrm{e}$

* vol * $9.35552 e+01 \mathrm{e}$

* elev * $5.556 \mathrm{e}$

*alp * $\quad$ a $1.0000 e+00 e$

* tr * $\quad$ * $3.3300 \mathrm{e}+02 \mathrm{e}$

*tv * $\quad$ t $3.3300 e+02 e$

* $p \quad$ f $3.41785 e+04 \mathrm{e}$

* pa *f $2.82800 e+04 e$

*

*

******* type num

valve

828

id ctitle

828 \$828\$ cavity vent valve 
Report:

Section:

Date:

Page:

ivty

icone

ivsv

ivtr

110

ivtrov

rvmx

$1.0000 e+01$

radin

$0.0000 e-00$

toutv

$3.3300 e+00$ rvov

$0.0000 e+00$

th

8.1800e-03

avlve

$3.2429 e-02$ nvtb1

fminov

$0.0000 e+00$

hout I

$0.0000 e+00$

hvive

2. 0320 e-01 jun2

829

ivps

epsw

$5.0000 e-05$

nvtb2

nvst

0

fmaxov

$1.0000 e+00$

houtv

$0.0000 e+00$

favlve

$0.0000 e+00$ tout 1

$3.3300 e+00$

xpos

$0.0000 e+00$

*

* dx * $\quad 1.0156 \mathrm{e}+00$ I2 $1.0000 \mathrm{e}+00 \mathrm{e}$

* vol * $1.0701 e+02$ r2 $7.2107 e-02 e$

* fa * $1.0536 \mathrm{e}+02$ r3 $7.2107 \mathrm{e}-02 \mathrm{e}$

* Eric *f $1.0000 \mathrm{e}-08 \mathrm{e}$

* elev * $6.50787 .5156 \quad 8.5156 \mathrm{e}$

* ha * $1.1582 \mathrm{e}+01$ r3 $3.0300 \mathrm{e}-01 \mathrm{e}$

* nff * r2 -1 r2 $1 e$

*alp *f $1.0000 e+00 e$

*v1 * $\quad$ v $0.0000 e+00 e$

$*$ vv *f $0.0000 e+00 e$

*t1 * E $3.3300 e+02 \mathrm{e}$

$*$ tv * E $3.3300 e+02 e$

$* \mathrm{p} \quad * \quad 3.41655 \mathrm{e}+04$

* pa * 2.82690e+04

$3.41622 \mathrm{e}+04 \quad 1.01340 \mathrm{e}+05 \mathrm{e}$

$2.82662 \mathrm{e}+04 \quad 9.76470 \mathrm{e}+04 \mathrm{e}$

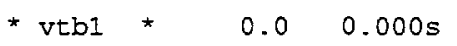

$*$ vtb1 * $0.20 .104 \mathrm{~s}$
*

* vtb1 * $0.4 \quad 0.352 \mathrm{~s}$

* $\mathrm{vtb1}$ * $0.6 \quad 0.648 \mathrm{~s}$

$\begin{array}{llll}* & \text { vtbl } & * 0.8 & 0.896 \mathrm{~s}\end{array}$

* vtb1 * $1.0 \quad 1.000 \mathrm{e}$

$\star$

$\star$

$\star \star \star \star \star \star \star$ type num

break 802

* junl ibty

829

0

volin

$1.0000 e+00$

pain

7.2107e-02

concin

$9.3946 \mathrm{e}+04$

$0.0000 e+00$

$1.0000 e+00$

rbmx

$1.0000 e+02$

id ctitle

$802 \$ 802 \$$ Cavity vent press. BC

isat

ioff

$\operatorname{tin}$

$3.3300 e+02$

poff

$0.0000 e+00$

$1.0133 e+05$

belv

$9.5156 e+00$

*

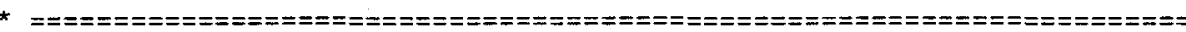

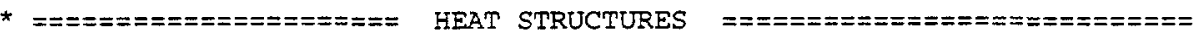

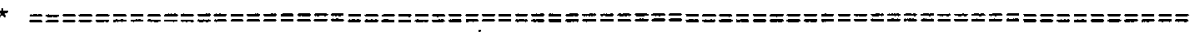

*

*

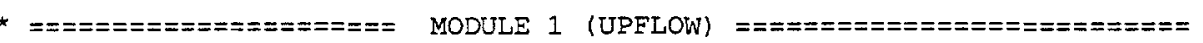

$\star$

* Upflow (Heated Al structure connected to water \& Cavity)

$\star * \star * * * * *$ type num id sitie

rod $901 \quad 901 \$ 901 \$$ He to Aluminum and then water

* nerx nerz ittc

* nopowr nridr modez liqlev iaxcnd 


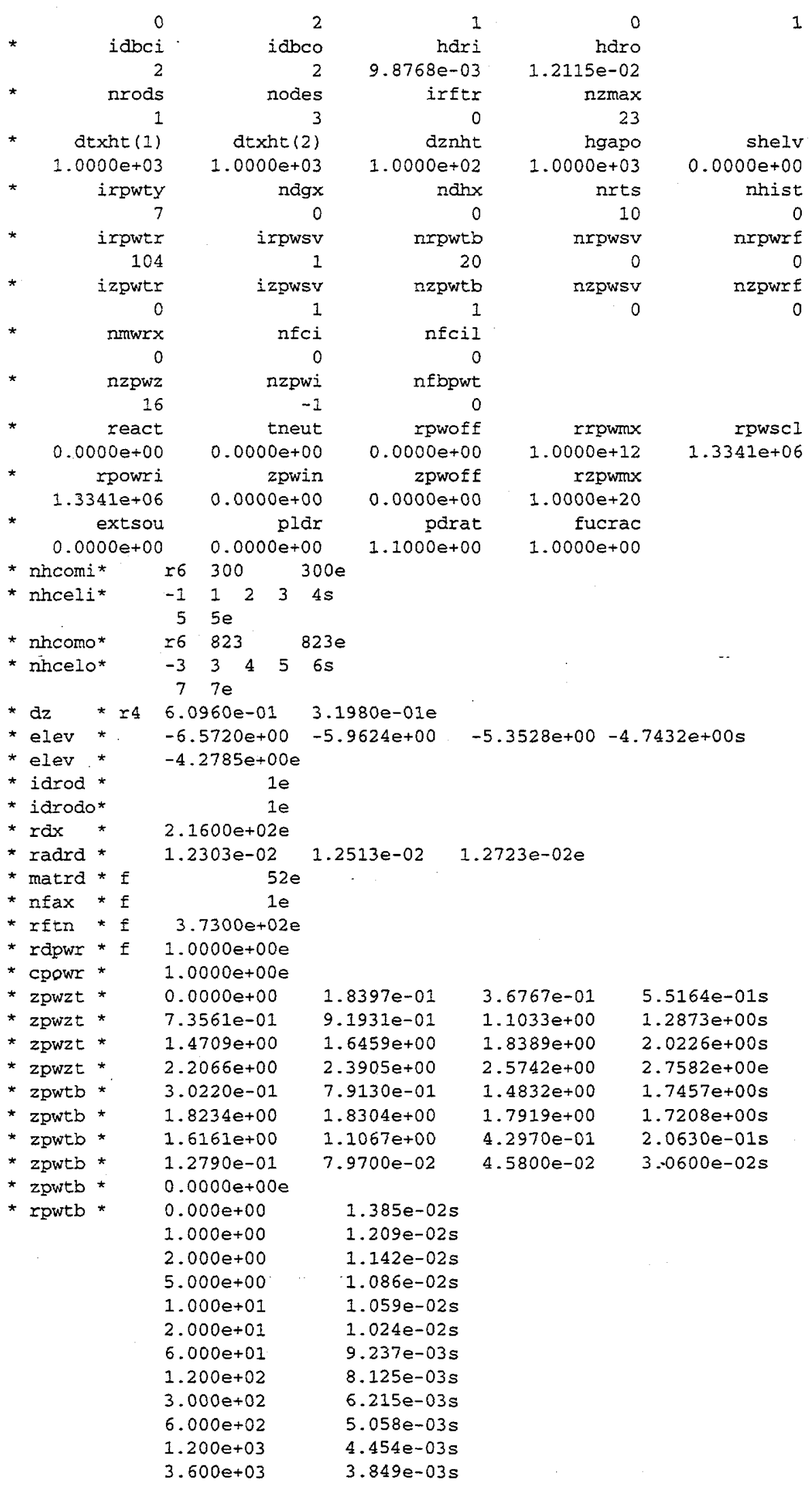




$\begin{array}{ll}7.200 e+03 & 3.457 e-03 s \\ 3.600 e+04 & 2.267 e-03 s \\ 8.640 e+04 & 1.472 e-03 s \\ 1.728 e+05 & 9.137 e-04 s \\ 4.320 e+05 & 4.449 e-04 s \\ 8.640 e+05 & 2.677 e-04 s \\ 1.728 e+05 & 1.664 e-04 s \\ 2.592 e+06 & 1.239 e-04 e\end{array}$

* fpuo 2 * $0.0000 e+00 e$

* itd * $1.0000 e+00 e$

* gmix * $1.0000 e+00 r 060.0000 e+00 e$

* gmles * $1.0000 e+00 e$

* pgapt * $1.0000 e+00 e$

* plvol * $1.0000 e+00 e$

* psien * $1.0000 e+00 e$

* clenn * $1.0000 e+00 e$

* burn * $1.0000 e+00 e$

*

$\star$

* Upflow (Heated Pb/Al structure connected to Water onIy)

$* \star * \star * \star *$ type num 0 id

$\begin{array}{rrr}\text { rod ncrx } & 951 & 951 \text { \$951\$ Blanket rowl htr } \\ * & \text { ncrz } & \text { ittc }\end{array}$

1

* nopowr nridr modez

* $\quad \begin{array}{rrr}0 & 1 & \text { har }\end{array}$

0
1
$i$

rrods

nodes

atxht (1)

1. $0000 \mathrm{e}+03$

irpwty

dtxht (2)

1. $0000 \mathrm{e}+03$

ndgx

irpwtr

104

izpwtr

irpwsv

izpwsv

1

nfci

nmwrx

nzpwi

$$
\text { nzpwz }
$$

16

react

0.0000 e+00

rpowri

1. $4157 \mathrm{e}+07$

extsou

$0.0000 \mathrm{e}+00$

tneut

$0.0000 \mathrm{e}+00$

zpwin

$0.0000 e+00$

plar

$0.0000 e+00$

* nhcomo* r6 300 300e

* nhcelo* $-1 \quad 1 \quad 2.345$

$* 2525.50$

*dz * $d z \quad 6.0960 e-01$

* elev * $\quad-6.5720 \mathrm{e}+00$

* elev * $\quad-4.7432 e+00$

* idrod * $\mathrm{f}$ ie

* $r d x * f \quad 2.8534 e+02 e$

* radra * $0.0000 e+00$

* radra * 1.0064 e-02

* matrd * r3 51 r2 $52 \mathrm{e}$

* nfax *

* retn * f $3.7300 e+02 e$

* rdpwr *f $1.0000 e+00 e$

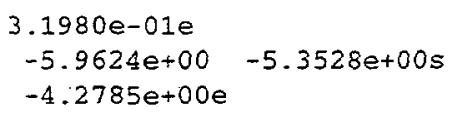

$0.0000 e+00$
irftr

Iiqlev

haro

2. 1164 e-02

nzmax

hgapo

1. 0000 e +03

nrts

10

nrpwsv

nzpwsv

0

nfcil

nfbpwt

rpwoff

$0.0000 e+00$

zpwoff

$0.0000 e+00$

pdrat

1. $1000 \mathrm{e}+00$

1. $0000 e+12$

rzpwinx

1. $0000 e+20$

fucrac

1. $0000 e+00$ 


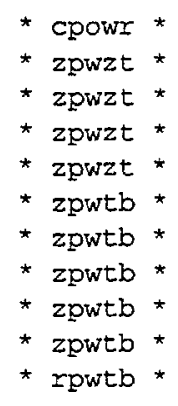

\section{$1.0000 e+00 e$ \\ $0.0000 e+00$}

$7.3561 e-01$

$1.8397 e-01$

$3.6767 e-01$

$5.5164 \mathrm{e}-01 \mathrm{~s}$

$1.4709 e+00$

$9.1931 e-01$

$1.1033 e+00$

$1.2873 \mathrm{e}+00 \mathrm{~s}$

$2.2066 e+00$

3. $0220 e-01$

1. $6459 e+00$

$1.8389 e+00$

$2.0226 \mathrm{e}+00 \mathrm{~s}$

$2.3905 e+00$

$2.5742 e+00$

$2.7582 \mathrm{e}+00 \mathrm{e}$

$7.9130 e-01$

$1.4832 \mathrm{e}+00$

$1.7457 \mathrm{e}+00 \mathrm{~s}$

$1.8234 \mathrm{e}+00$

$1.8304 e+00$

$1.7919 e+00$

$1.7208 \mathrm{e}+00 \mathrm{~s}$

$1.6161 \mathrm{e}+00$

$1.1067 \mathrm{e}+00$

$4.2970 \mathrm{e}-01$

$2.0630 \mathrm{e}-01 \mathrm{~s}$

$1.2790 e-01$

$0.0000 e+00 e$

$0.000 e+00$

$1.000 e+00$

$2.000 e+00$

$5.000 e+00$

$1.000 e+01$

$2.000 e+01$

$6.000 e+01$

1. $200 e+02$

$3.000 e+02$

$6.000 e+02$

1. $200 \mathrm{e}+03$

$3.600 \mathrm{e}+03$

$7.200 e+03$

$.9700 \mathrm{e}-02$

$4.5800 \mathrm{e}-02$

$3.0600 \mathrm{e}-02 \mathrm{~s}$

3. $600 e+04$

$8.640 e+04$

1. $728 \mathrm{e}+05$

$4.320 e+05$

8. $640 e \div 05$

$1.385 \mathrm{e}-02 \mathrm{~s}$

$1.209 \mathrm{e}-02 \mathrm{~s}$

$1.142 \mathrm{e}-02 \mathrm{~s}$

$1.086 \mathrm{e}-02 \mathrm{~s}$

$1.059 \mathrm{e}-02 \mathrm{~s}$

$1.024 \mathrm{e}-02 \mathrm{~s}$

$9.237 e-03 s$

$8.125 \mathrm{e}-03 \mathrm{~s}$

$6.215 e-03 s$

$5.058 \mathrm{e}-03 \mathrm{~s}$

$4.454 \mathrm{e}-03 \mathrm{~s}$

$3.849 e-03 s$

$3.457 \mathrm{e}-03 \mathrm{~s}$

$2.267 \mathrm{e}-03 \mathrm{~s}$

$1.472 \mathrm{e}-03 \mathrm{~s}$

$9.137 \mathrm{e}-04 \mathrm{~s}$

$4.449 \mathrm{e}-04 \mathrm{~s}$

$2.677 \mathrm{e}-04 \mathrm{~s}$

$1.728 e+06$

1. $664 \mathrm{e}-04 \mathrm{~s}$

$2.592 e+06$

* fpuo $2 * \quad 0.0000 e+00 e$

$1.239 \mathrm{e}-04 \mathrm{e}$

$*$ ftd * $1.0000 e+00 e$

* gmix * $1.0000 e+00 r 060.0000 e+00 e$

* gmies * $1.0000 e+00 e$

* pgapt * $1.0000 e+00 e$

* plvol * $1.0000 e+00 e$

* pslen * $1.0000 e+00 e$

* clenn * $1.0000 e+00 e$

* burn *f $1.0000 e+00 e$

$\star$

*

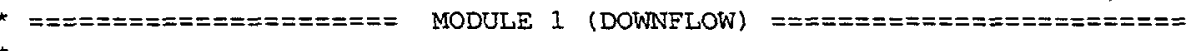

*

* Downflow (Heated Al structure connected to Water \& Cavity)

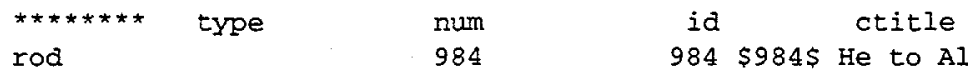

* ncrx ncrz

* nopowr $\quad \begin{array}{r}1 \\ \text { nriar }\end{array}$

* $\quad \begin{array}{rr}0 & 2 \\ \text { idbci } & \text { idbco }\end{array}$

nrods

nodes

1

dtxht (1)

$1.0000 e+03$

irpwty

dtxht (2)

$1.0000 e+03$

ndgx

irpwtr

104

izpwtr

irpwsv

izpwsv

$984 \$ 984 \$$ He to Aluminum and then water ittc

0

modez

1

$9.8768 e-03$

irftr

0

dznht

$1.0000 e+02$

ndhx

nrpwtb

20

nzpwtb

1

liqlev
0
hdro
$1: 2115 e-02$
nzmax
23
ngapo
$1.0000 \mathrm{e}+03$
nrts
10
nrpwsv
0
nzpwsv
0

iaxcnd

1

shelv

0.0000 e +00

nhist

nxpwrf

nzpwrt

0

0 
* nmwrx

* nzpwz

16

$* \quad$ react

$0.0000 e+00$

rpowri

$8.2220 e+06$

extsou

$0.0000 e+00$

* nhcomi* $r 6$

* nhceli*

* nhcomo*

* nhcelo*

* az

* elev *

* elev *

* idrod *

* idrodo*

* rdx

* radra *

* matrd * $f$

* nfax *f

$* \operatorname{rftn} * f$

* rdpwr * $f$

* cpowr *

* zpwzt *

* zpwzt *

* zpwzt *

* zpwzt *

* zpwtb *

* zpwtb *

* zpiwtb *

* zpwtb *

* zpwtb *

* rpwtb *
* fpuo2 *

* fta *

* gmix *

* gimies *

* pgapt *

$$
\text { nfci }
$$

nzpwi

$-1$

$$
0.0000 e+00
$$$$
z \text { pwin }
$$

$0.0000 e+00$

plar

$0.0000 e+00$

r6 360

$\begin{array}{lll}-1 & 1 & 2\end{array}$

$55 e$

r6 823

$\begin{array}{llll}-7 & 765 & 45\end{array}$

$33 \mathrm{e}$

$$
3.1980 \mathrm{e}-01 \times 4
$$

$-4.2785 e+00$

$-5.9624 e+00$

$1 e$

$1 e$

$6.1183 e+02 e$

$4.9384 e-03$ $52 \mathrm{e}$

$1 \mathrm{e}$

$3.7300 e+02 e$

$1.0000 e+00 e$

$1.0000 \mathrm{e}+00 \mathrm{e}$

$0.0000 e+00$

$7.3561 e-01$

$1.4709 e+00$

$2.2066 \mathrm{e}+00$

3. $0220 \mathrm{e}-01$

$1.8234 e+00$

$1.6161 e+00$

1. $2790 \mathrm{e}-01$

$0.0000 e+00 e$

$0.000 e+00$

$1.000 e+00$

$2.000 e+00$

$5.000 e+00$

$1.000 e+01$

$2.000 e+01$

$6.000 e+01$

1. $200 \mathrm{e}+02$

$3.000 e+02$

$6.000 e+02$

I. $200 e+03$

$3.600 e+03$

$7.200 \mathrm{e}+03$

$3.600 e+04$

$8.640 e+04$

1. $728 e+05$

$4.320 e+05$

$8.640 e+05$

$1.728 \mathrm{e}+06$

$2.592 \mathrm{e}+06$

$0.0000 e+00 e$

$1.0000 e+00 e$

$1.0000 e+00 r 06 \quad 0.0000 e+00 e$

$1.0000 \mathrm{e}+00 \mathrm{e}$

$1.0000 \mathrm{e}+00 \mathrm{e}$

$5.4980 e-03$

$1.8397 e-01$

$9.1931 e-01$

$1.6459 e+00$

$2.3905 e+00$

$7.9130 e-01$

$1.8304 \mathrm{e}+00$

$1.1067 e+00$

$7.9700 \mathrm{e}-02$

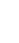

$6.0960 e-01 e$

$-4.7432 e+00$

$-6.5720 \mathrm{e}+00 \mathrm{e}$

$6.0576 e-03 e$

$3.6767 e-01$

$5.5164 \mathrm{e}-01 \mathrm{~s}$

$1.1033 e+00$

$1.8389 \mathrm{e}+00$

$2.5742 e+00$

$1.4832 \mathrm{e}+00$

1. $7919 \mathrm{e}+00$

$4.2970 \mathrm{e}-01$

$4.5800 e-02$

$1.2873 e+00 \mathrm{~s}$

$2.0226 \mathrm{e}+00 \mathrm{~s}$

$2.7582 \mathrm{e}+00 \mathrm{e}$

$1.7457 \mathrm{e}+00 \mathrm{~s}$

$1.7208 \mathrm{e}+00 \mathrm{~s}$

$2.0630 e-01 s$

$1.385 \mathrm{e}-02 \mathrm{~s}$

$1.209 \mathrm{e}-02 \mathrm{~s}$

$1.142 \mathrm{e}-02 \mathrm{~s}$

$1.086 \mathrm{e}-02 \mathrm{~s}$

$1.059 \mathrm{e}-02 \mathrm{~s}$

$1.024 \mathrm{e}-02 \mathrm{~s}$

$9.237 \mathrm{e}-03 \mathrm{~s}$

$8.125 \mathrm{e}-03 \mathrm{~s}$

$6.215 e-03 s$

$5.058 \mathrm{e}-03 \mathrm{~s}$

$4.454 \mathrm{e}-03 \mathrm{~s}$

$3.849 e-03 \mathrm{~s}$

$3.457 \mathrm{e}-03 \mathrm{~s}$

$2.267 \mathrm{e}-03 \mathrm{~s}$

$1.472 e-03 \mathrm{~s}$

$9.137 e-04 \mathrm{~s}$

$4.449 \mathrm{e}-04 \mathrm{~s}$

$2.677 \mathrm{e}-04 \mathrm{~s}$

$1.664 \mathrm{e}-04 \mathrm{~s}$

1. $239 \mathrm{e}-04 \mathrm{e}$ 
* plvol * $1.0000 e+00 e$

* pslen * $1.0000 e+00 e$

* clenn * $1.0000 e+00 e$

* burn * E $1.0000 e+00 e$

*

*

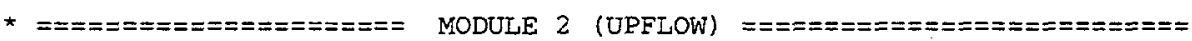

* Upflow (Unheated Al structure connected to water \& Cavity)

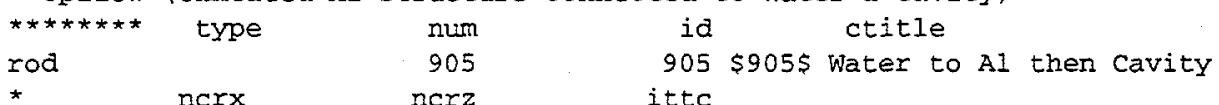

rod

$\begin{aligned} \operatorname{ncrx} & \text { ner } \\ 1 & \end{aligned}$

nopow

nriar

idbci

2

idbco

nrods

2

nodes

$\operatorname{dtxht}(1)$

dexht (2)

$1.0000 e+03$

$1.0000 e+03$

modez

hari
$1.0210 e-02$

liqlev

iaxend

irftr

$5.3477 e-02$

nemax

dznht

$1.0000 e+02$

hgapo

$1.0000 e+03$

shelv

* nincomi* $\quad$ 7 $102 \quad 102$

* nhceli* $\quad-1 \quad 1 \quad 2 \quad 3 \quad 4 \mathrm{~s}$

* nhceli* $566 \mathrm{6e}$

* nhcomo* r7 $823 \quad 823 \mathrm{e}$

* nhcelo* $-2 \quad 2 \quad 34 \mathrm{~s}$

* nhcelo* $567 \mathrm{7e}$

*dz * 0.7418 r4

$*$ elev * $\quad-7.2477 e+00$

* elev * $*-5.3528 e+00$

$0.6096 \quad 0.3198 \mathrm{e}$

* idrod *

* idrodo*

* rax *

* radrd *

* matrd * $E$

* nfax *

* rftr * $f$

$-6.5720 e+00$

$-5.9624 e+00 s$

$1 e$

$-4.7432 \mathrm{e}+00$

$-4.2785 e+00 e$

$3.5325 e+02 e$

$1.6882 \mathrm{e}-02 \quad 1.7076 \mathrm{e}-02 \quad 1.7269 \mathrm{e}-02 \mathrm{e}$

$52 e$

$1 e$

$3.7300 e+02 e$

*

* Upflow (Heated $\mathrm{Pb} / \mathrm{Al}$ structure connected to water only)

$* * * * * * * \quad$ typ

rod

nerx

num

ctitle

* ner

1

nopowr

idbci

0

nrods

1

dtxht (1)

1. $0000 e+03$

irpwty

irpwtr

104

izpwtr

nerz

6

nridr

1

idbco

nodes

dexht (2)

1. $0000 e+03$

ndgx

irpwsv

izpwsv

1

nfci

nmwrx

nzpwz

16

nzpwi

$-1$

$$
\begin{array}{r}
\text { id } \\
955 \\
\text { ittc } \\
0 \\
\text { modez } \\
1 \\
\text { hdri } \\
0.0000 e+00 \\
\text { irftr } \\
0 \\
\text { dznht } \\
1.0000 e+02 \\
\text { ndhx } \\
0 \\
\text { nrpwtb } \\
20 \\
\text { nzpwtb } \\
1 \\
\text { nfeil } \\
0 \\
\text { nfbpwt }
\end{array}
$$


Report:

Section:

Date:

Page:

rpwscI

react

rpowri

$7.6600 e+06$

extsou

$$
\begin{array}{lr}
0.0000 e+00 \\
r 7102 \quad 102 e
\end{array}
$$

$4566 e$

$0.7418 \quad r 4$
$1.0003 e-02$

$1.5005 e-02 \mathrm{~s}$

4. $6655 e-01$

$1.4000 e+00$

$2.3335 e+00$

$3.2666 e+00$

$3.4800 e-02$

$2.2734 e+00$

$2.0159 e+00$

$7.2700 e-02$

$7.0000 \mathrm{e}-01 \mathrm{~s}$

$1.6335 \mathrm{e}+00 \mathrm{~s}$

2. $5666 \mathrm{e}+00 \mathrm{~s}$

$3.5000 \mathrm{e}+00 \mathrm{e}$

$1.6680 \mathrm{e}-01 \mathrm{~s}$

$2.4080 e+00 s$

$8.2910 \mathrm{e}-01 \mathrm{~s}$

$4.0200 \mathrm{e}-02 \mathrm{~s}$
$0.0000 e+00$

* nhcomo*

* nicelo*

* nhcelo*

* $\mathrm{dz}$ *

* elev *

* elev *

* idrod * $\mathrm{E}$

* $\mathrm{rdx} * \mathrm{f}$

* radara *

* radra *

* matra * r3

* nfax * $f$

$* \operatorname{rftn} * \mathrm{f}$

* rapwr * $f$

* cpowr *

* zpwzt *

* zpwzt *

* zpwzt *

* zpwzt *

* zpwtb *

* zpwtb *

* zpwtb *

* zpwtb *

* zpwtb *

* rpwtb *

$102 \quad 102 e$

$\begin{array}{lll}-1 & 1 & 2.3 \mathrm{~s}\end{array}$

$-7.2477 \mathrm{e}+00$

$-5.3528 e+00$

Ie

$3.5325 e+02 e$

$0.0000 e+00$

$1.5497 e-02$

51 r2 $52 \mathrm{e}$

$3.7300 e+02 e$

$1.0000 \mathrm{e}+00 \mathrm{e}$

$1.0000 \mathrm{e}+00 \mathrm{e}$

$0.0000 e+00$

$9.3345 e-01$

$1.8666 e+00$

$2.8000 e+00$

$0.0000 e+00$

$4.7850 e-01$

$2.3795 e+00$

$2.7530 e-01$

$0.0000 \mathrm{e}+00 \mathrm{e}$

$0.000 e+00$

$1.000 e+00$

$2.000 e+00$

$5.000 e+00$

$1.000 e+01$

$2.000 e+01$

$6.000 e+01$

$1.200 e+02$

3. $000 e+02$

$6.000 e+02$

- $1.200 e+03$

$3.600 e+03$

$7.200 e+03$

$3.600 e+04$

$8.640 e+04$

$1.728 e+05$

$4.320 e+05$

$8.640 e+05$

$1.728 e+06$

$2.592 e+06$

* Epuo2 *

$0.0000 e+00 e$

* ftd *

* gmix *

* gmles *

* pgapt *

* plvol *

* pslen *

* clenn *

*burn * $f$

$1.0000 \mathrm{e}+00 \mathrm{e}$

$1.0000 e+00 r 060.0000 e+00 e$

$1.0000 \mathrm{e}+00 \mathrm{e}$

$1.0000 e+00 e$

$1.0000 \mathrm{e}+00 \mathrm{e}$

$1.0000 \mathrm{e}+00 \mathrm{e}$

$1.0000 \mathrm{e}+00 \mathrm{e}$

$1.0000 e+00 e$

$1.4430 e-01$

$6.317 \mathrm{e}-04 \mathrm{~s}$
$0.6096 \quad 0.3198 \mathrm{e}$

$-6.5720 e+00-5.9624 e+00 s$

$-4.7432 e+00-4.2785 e+00 e$

$5.0017 e-03$

$1.5989 \mathrm{e}-02 \mathrm{e}$

$2.3345 e-01$

1. $1666 e+00$

$2.1000 e+00$

$3.0334 e+00$

$1.1700 e-02$

$1.5993 e+00$

$2.2644 e+00$

$2.079 e-02 s$

1. $747 \mathrm{e}-02 \mathrm{~s}$

$1.614 \mathrm{e}-02 \mathrm{~s}$

$1.511 e-02 \mathrm{~s}$

$1.477 \mathrm{e}-02 \mathrm{~s}$

$1.432 \mathrm{e}-02 \mathrm{~s}$

$1.293 \mathrm{e}-0.2 \mathrm{~s}$

$1.135 e-02 s$

$8.651 \mathrm{e}-03 \mathrm{~s}$

$7.042 e-03 \mathrm{~s}$

$6.255 \mathrm{e}-03 \mathrm{~s}$

$5.490 \mathrm{e}-03 \mathrm{~s}$

$4.950 \mathrm{e}-03 \mathrm{~s}$

$3.271 \mathrm{e}-03 \mathrm{~s}$

$2.169 e-03 \mathrm{~s}$

$1.377 \mathrm{e}-03 \mathrm{~s}$

$3.457 \mathrm{e}-04 \mathrm{~s}$

$2.046 \mathrm{e}-04 \mathrm{~s}$

$1.488 \mathrm{e}-04 \mathrm{e}$
WSRC-TR-98-0053

Appendix A

07/14/98

78 of 96

$\star$

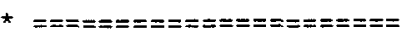

MODULE 2 (DOWNFLOW)

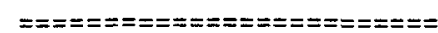


* Downflow (Unheated Al str

$\begin{array}{lrrr}* * * * * * * & \text { type } & \text { num } & \text { id } \\ \text { rod } & & 916 & 916 \\ * & \text { ncrx } & \text { ncrz } & \text { ittc } \\ * & 1 & 6 & 0 \\ * & \text { nopowr } & \text { nridr } & \text { modez } \\ * & 1 & 2 & 1 \\ & \text { idbci } & \text { idbco } & \text { hdri } \\ * & 2 & 2 & 1.0210 \text { e-02 } \\ & \text { nrods } & \text { nodes } & \text { irftr } \\ * & \text { dtxht }(1) & \text { dtxht }(2) & 0\end{array}$

cted to Water \& Cavity)

$00000+03$

$1.0000 e+02$

ctitle

nhcomi* r7 $158 \quad 158 \mathrm{e}$

* nhceli* $\quad-1 \quad 1 \quad 234 \mathrm{~s}$

* nhceli*

* nhcomo*

$566 \mathrm{e}$

17823

823 e

* nhcelo*

$\begin{array}{llllll}-7 & 7 & 6 & 5 & 4 & \mathrm{~s}\end{array}$

* nhcelo*

* dz *

$322 e$

$0.3198 \quad r 4$

$-4.2785 e+00$

0.5096

$0.7418 \mathrm{e}$

* elev *

$-5.9624 e+00$

$-4.7432 e+00 \quad-5.3528 e+00 s$

* jarod *

$-6.5720 e+00 \quad-7.2477 e+00 e$

* idrodo*

le

le

* rdx

$1.8472 \mathrm{e}+02 \mathrm{e}$

* radra *

2. $6740 \mathrm{e}-02$

$2.6928 e-02 \quad 2.7115 e-02 e$

* matra * $E$

* nfax *E

$* \operatorname{rftn} * f$

$52 \mathrm{e}$

$1 e$

$3.7300 \mathrm{e}+02 \mathrm{e}$

* Downflow (Heated Pb/AI structure connected to Water only)

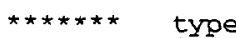

rod

* nerx

* nopowr 1

nopowr

* $\quad$ idbci

* $\quad \begin{array}{r}0 \\ \text { nrods }\end{array}$

dtxht (I)

$1.0000 e+03$

irpwty

irpwtr

104

izpwtr

nmwrx

0

nzpwz

react

$0.0000 e+00$

rpowri

3. $0600 \mathrm{e}+06$

extsou

$0.0000 e+00$

* nhcomo* num

966

nerz

6

1

2

nodes

dexht (2)

1.0000 e+03

ndg $x$

irpwsv

1

izpwsv

nfei

nzpwi

$-1$

tneut

$0.0000 e+00$

zpwin

$0.0000 e+00$

plar

$0.0000 e+00$

r7 158

158 e ctitle

$966 \$ 966 \$ \mathrm{~Pb} / \mathrm{Al}$ to Water

ittc

modez

1

0.0000 et00

irftr

aznht

1. $0000 \mathrm{e}+02$

$\operatorname{ndhx}$

nrpwtb

20

nzpwtb

0

nfbowt

rpwoff

$0.0000 e+00$

zpwoff

0.0000 e+00

parat

$1.1000 \mathrm{e}+00$

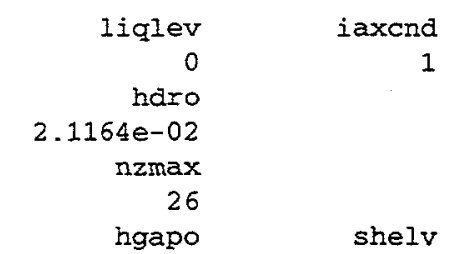

1. $0000 \mathrm{e}+03$

nrts

10

nrpwsv

0

rrpwmx

1. $0000 \mathrm{e}+12$

rzpwmx

1. $0000 \mathrm{e}+20$

fucrac

1. $0000 \mathrm{e}+00$
$0.0000 e+00$

nhist

nrpwref

nzpwrf

0

rpwscl

$3.0600 \mathrm{e}+06$ 
* nhcelo*

* $\mathrm{d} z$

* elev *

* eler *

* idrod * $\mathrm{f}$

* rdx * $\mathrm{f}$

* radra *

* radrd *

* matra * $r$

* nfax *f

* rftr *

* rdpwr *f

* cpowr *

* zpwzt *

* zpwzt *

* zpwzt *

* zpwzt *

* zpwtb *

* zpwtb *

* zpwtb *

* zpwtb *

* zpwtb *

* rpwtb *

$$
\begin{array}{rrr}
-1 & 1 & 2 \\
5 & 6 & 6 e \\
0.3198
\end{array}
$$

$-4.2785 e+00$

$-5.9624 e+00$

1 e

$1.8472 \mathrm{e}+02 \mathrm{e}$

$0.0000 e+00$

$2.5033 e-02$

$51 \times 2 \quad 52 \mathrm{e}$

3. $7300 e+02$

$1.0000 e+00 \mathrm{e}$

$1.0000 \mathrm{e}+00 \mathrm{e}$

$0.0000 e+00$

$9.3345 e-01$

$1.8666 \mathrm{e}+00$

$2.8000 e+00$

$0.0000 e+00$

4.7850 e- 01

$2.3795 e+00$

$2.7530 \mathrm{e}-01$

$0.0000 e+00 e$

$0.000 e+00$

$1.000 e+00$

$2.000 e+00$

$5.000 e+00$

$1.000 e+01$

$2.000 e+01$

$6.000 e+01$

1. $200 \mathrm{e}+02$

$3.000 e+02$

$6.000 e+02$

1. $200 e+03$

$3.600 e+03$

$7.200 e+03$

$3.600 e+04$

$8.640 \mathrm{e}+04$

1. $728 \mathrm{e}+05$

$4.320 e+05$

- 8.640e+05

$1.728 e+06$

$2.592 \mathrm{e}+06$

* fpuo2 *

$0.0000 e+00 e$

* ftd *

* $\operatorname{gmix}$ *

* gmles *

* pgapt *

* plvol *

* psien *

* clenn *

* burn *f

$1.0000 e+00 e$

$1.0000 e+00 r 06 \quad 0.0000 e+00 e$

$1.0000 e+00 e$

$1.0000 \mathrm{e}+00 \mathrm{e}$

$1.0000 e+00 \mathrm{e}$

$1.0000 e+00 e$

$1.0000 e+00 e$

$1.0000 \mathrm{e}+00 \mathrm{e}$

4. $6655 e-01$

$1.4000 e+00$

$2.3335 e+00$

$3.2666 e+00$

$3.4800 e-02$

$2.2734 e+00$

$2.0159 e+00$

$7.2700 e-02$

2. $644 e+00$

1. $4430 e-01$

$2.801 \mathrm{e}-02 \mathrm{~s}$

$2.370 \mathrm{e}-02 \mathrm{~s}$

$2.197 \mathrm{e}-02 \mathrm{~s}$

$2.061 e-02 \mathrm{~s}$

$2.014 \mathrm{e}-02 \mathrm{~s}$

$1.947 \mathrm{e}-02 \mathrm{~s}$

$1.730 \mathrm{e}-02 \mathrm{~s}$

$1.482 \mathrm{e}-02 \mathrm{~s}$

$1.059 \mathrm{e}-02 \mathrm{~s}$

$8.183 e-03 s$

$7.174 \mathrm{e}-03 \mathrm{~s}$

$6.370 \mathrm{e}-03 \mathrm{~s}$

$5.783 \mathrm{e}-03 \mathrm{~s}$

$3.872 e-03 s$

2. $573 e-03 s$

1. $618 \mathrm{e}-03 \mathrm{~s}$

$6.990 \mathrm{e}-04 \mathrm{~s}$

$3.595 e-04 \mathrm{~s}$

$2.088 \mathrm{e}-04 \mathrm{~s}$

1. $516 \mathrm{e}-04 \mathrm{e}$

*

*

*

* Upflow (Unheated Al structure connected to water \& Cavity)

$* * * * * * *$ type num id ctitle

rod 911

* nerx nerz

15

* nopowr

nriar

911 \$911\$Fe to Aluminum and then water ittc

0

modez

iaxcnd

$7.0000 e-015$

$35 e+00 s$

$5000 e+00 e$

8. $2910 e-01 s$

$4.0200 e-02 s$ 
Report:

Section:

Date:

Page:
WSRC-TR-98-0053

Appendix A

07/14/98

81 of 96

TRAC BLANKET SYSTEM MODEL

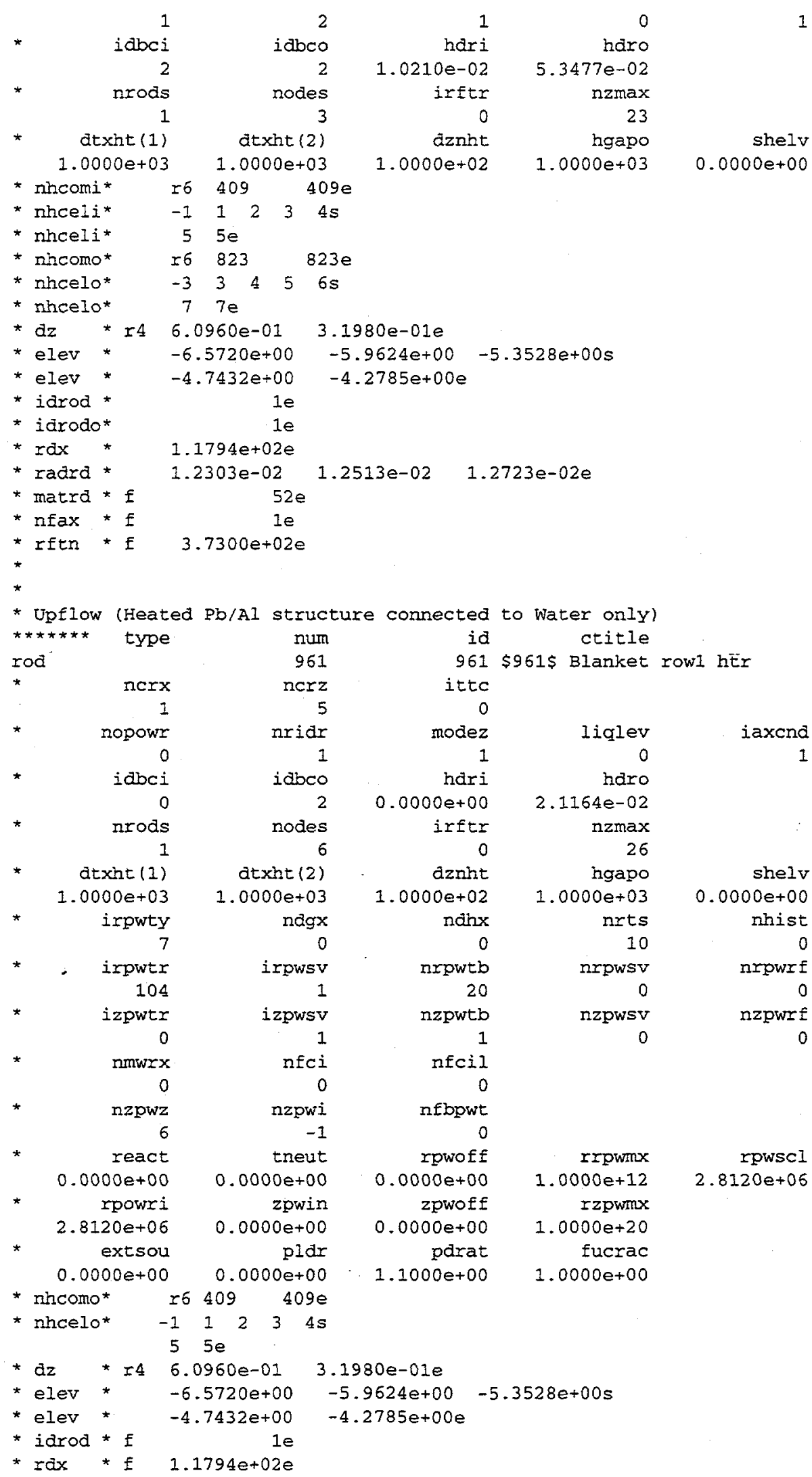




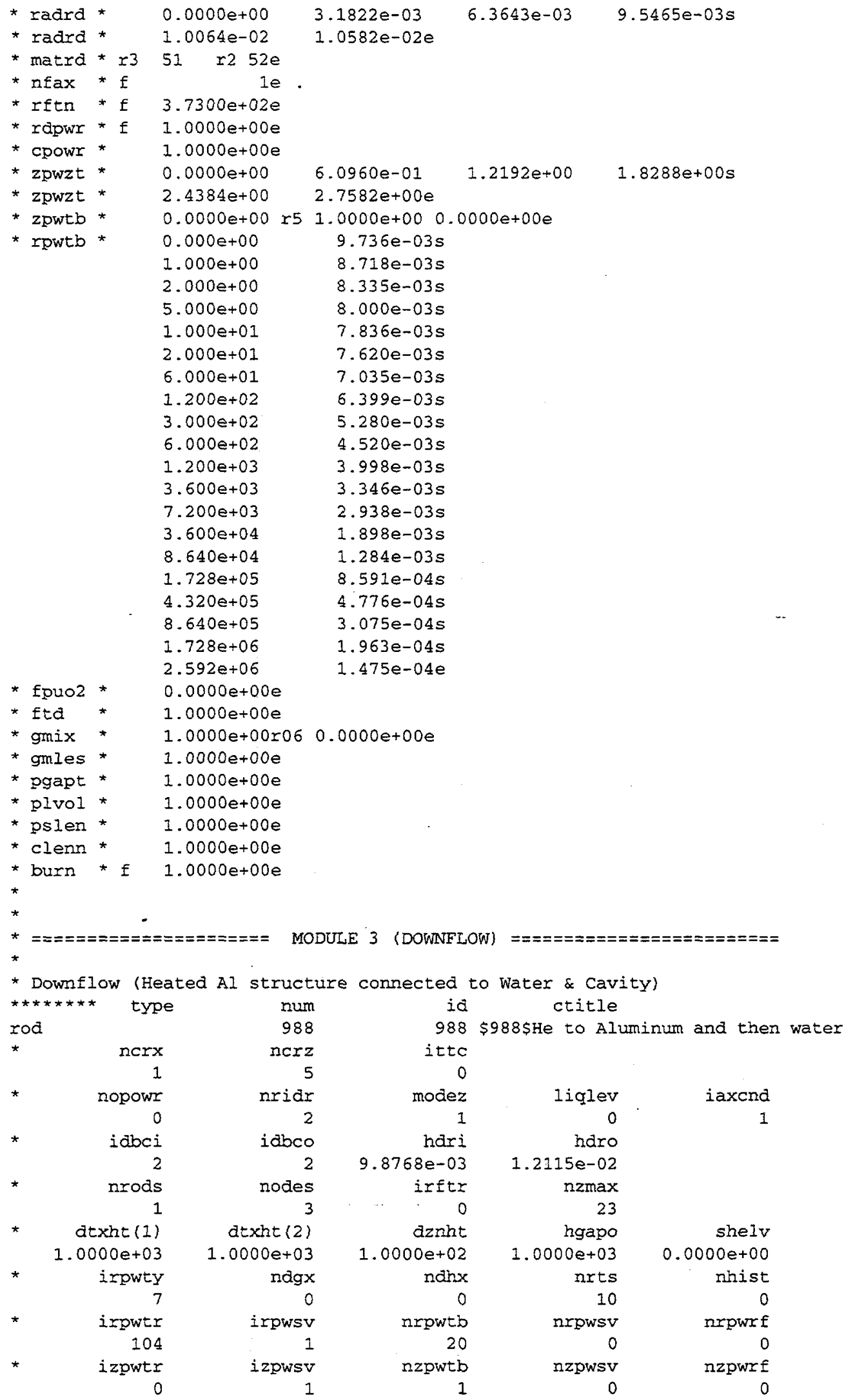




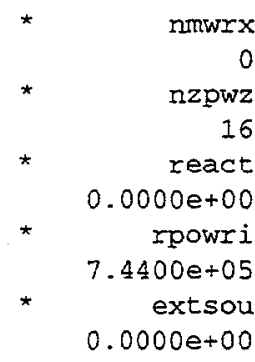

nfci
0
nzpwi
-1
tneut

$0.0000 e+00$

zpwin

$0.0000 e+00$

pldr

$0.0000 e+00$

* nhcomi*

* nhceli*

* nhcomo*

* nhcelo*

$\star \mathrm{dz} \quad$ *

* elev *

* elev *

* idrod *

* idrodo*

* rax

* radrd *

* matra * $f$

* Iftr *f

* rdpwr * $f$

* cpowr *

* zpwzt *

* zpwzt *

* zpwzt *

* zpwzt *

* zpwtb *

* zpwtb *

* zpwtb *

* zpwtb *

* zpwtb *

* rpwtb *

r6 478

$55 e$

г6 823

$33 \mathrm{e}$

$3.1980 e-01$ r 4

$-4.2785 e+00$

$-5.9624 e+00$

$1 e$

$6.1183 e+02 e$

$4.9384 e-03$ $52 e$

$1 \mathrm{e}$

$3.7300 e+02 e$

I. $0000 \mathrm{e}+00 \mathrm{e}$

$0.0000 e+00$

$7.3561 e-01$

$1.4709 e+00$

$2.2066 e+00$

$3.0220 e-01$

$1.8234 \mathrm{e}+00$

$1.6161 \mathrm{e}+00$

$1.2790 \mathrm{e}-01$

$0.000 e+00$
* nfax * $f$

$\begin{array}{lllll}-1 & 1 & 2 & 3 & 45\end{array}$

$\begin{array}{rlllll}-7 & 7 & 6 & 5 & 4 & \mathrm{~s}\end{array}$

$6.0960 \mathrm{e}-01 \mathrm{e}$ $-4.7432 e+00$ $-6.5720 e+00 e$

$$
5.4980 e-03
$$

$1.0000 e+00 \mathrm{e}$

$0.0000 e+00 e$

$1.000 e+00$

$2.000 e+00$

$5.000 e+00$

$1.000 e+01$

$2.000 e+01$

$6.000 e+01$

1. $200 e+02$

3. $000 e+02$

$6.000 e+02$

1. $200 \mathrm{e}+03$

3. $600 e+03$

7. $200 e+03$

3. $600 \mathrm{e}+04$

$8.640 e+04$

1. $728 \mathrm{e}+05$

$4.320 e+05$

$8.640 \mathrm{e}+05$

1. $728 \mathrm{e}+06$

$2.592 e+06$

* fpuo2 *

$0.0000 \mathrm{e}+00 \mathrm{e}$

* fta * $1.0000 e+00 e$

* gmix *

* gmies *

* pgapt *
$1.0000 \mathrm{e}+00 \mathrm{r} 06 \quad 0.0000 \mathrm{e}+00 \mathrm{e}$

$1.0000 \mathrm{e}+00 \mathrm{e}$

$1.0000 \mathrm{e}+00 \mathrm{e}$
$1.8397 \mathrm{e}-01$
$9.1931 \mathrm{e}-01$
1. $6459 \mathrm{e}+00$
$2.3905 e+00$
$7.9130 e-01$
$1.8304 \mathrm{e}+00$
$1.1067 e+00$
$7.9700 \mathrm{e}-02$
$9.736 \mathrm{e}-03 \mathrm{~s}$
$8.718 \mathrm{e}-03 \mathrm{~s}$
$8.335 e-03 \mathrm{~s}$
$8.000 e-03 s$
$7.836 \mathrm{e}-03 \mathrm{~s}$
$7.620 e-03 s$
$7.035 \mathrm{e}-03 \mathrm{~s}$
$6.399 \mathrm{e}-03 \mathrm{~s}$
$5.280 \mathrm{e}-03 \mathrm{~s}$
$4.520 \mathrm{e}-03 \mathrm{~s}$
$3.998 \mathrm{e}-03 \mathrm{~s}$
$3.346 \mathrm{e}-03 \mathrm{~s}$
$2.938 \mathrm{e}-03 \mathrm{~s}$
$1.898 \mathrm{e}-03 \mathrm{~s}$
$1.284 \mathrm{e}-03 \mathrm{~s}$
$8.591 \mathrm{e}-04 \mathrm{~s}$
$4.776 \mathrm{e}-04 \mathrm{~s}$
$3.075 \mathrm{e}-04 \mathrm{~s}$
$1.963 \mathrm{e}-04 \mathrm{~s}$
$1.475 \mathrm{e}-04 \mathrm{e}$

$3.6767 e-01$

$1.1033 e+00$

$1.8389 e+00$

$2.5742 e+00$

$1.4832 e+00$

$1.7919 e+00$

$4.2970 e-01$

$4.5800 e-02$

$5.5164 \mathrm{e}-01 \mathrm{~s}$

$1.2873 \mathrm{e}+00 \mathrm{~s}$

$2.0226 \mathrm{e}+00 \mathrm{~s}$

$2.7582 \mathrm{e}+00 \mathrm{e}$

$1.7457 \mathrm{e}+00 \mathrm{~s}$

$1.7208 \mathrm{e}+00 \mathrm{~s}$

$2.0630 \mathrm{e}-01 \mathrm{~s}$

$3.0600 \mathrm{e}-02 \mathrm{~s}$ $\begin{array}{rr}\text { rrpwmx } & \text { rpwscl } \\ 1.0000 e+12 & 7.4400 e+05 \\ \text { rzpwmx } & \end{array}$

Eucrac

$1.0000 e+00$ 
* plvol * $1.0000 e+00 e$

* pslen * $1.0000 e+00 e$

* clenn * $1.0000 e+00 e$

* burn * e $1.0000 e+00 e$

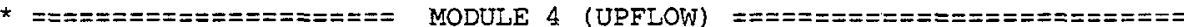

*

* Upflow (Unheated AI structure connected to water \& Cavity)

$\begin{array}{llll}* * * * * * * * & \text { num } & \text { id } & \text { ctitle } \\ \text { rod } & 912 & 912 \$ 912 \$ \mathrm{He} \text { to All }\end{array}$

$912 \quad 912 \$ 912 \$ \mathrm{He}$ to Aluminum and then water

* nerx ncrz ittc

* nopowr nridr

* $\quad \begin{array}{rr}1 & 2 \\ \text { *dbci } & \text { idbco }\end{array}$

modez

dez

Iiqlev iaxcnd

* nroas

nroas nodes

nodes

hari

haro

1.0210 e-02

$5.3477 e-02$

irftr

nzmax

* $\operatorname{atxht(1)}$

atxht (2)

1. $0000 e \div 03$

1. $0000 \mathrm{e}+03$

aznit

1. $0000 \mathrm{e}+02$

hgapo

1. $0000 e+03$

shelv

* nhcomi* r7 $412 \quad 412 \mathrm{e}$

* nhceli* $\quad-1 \quad 1 \quad 2 \quad 34 \mathrm{~s}$

* nhceli* 566 e

* nhcomo* r7 $823 \quad 823 \mathrm{e}$

* nhcelo* $\quad-2 \quad 234 \mathrm{~s}$

* nicelo* $5677 \mathrm{fe}$

* $\mathrm{dz} \quad$ * $0.7418 \quad r 4$

* elev * $\quad-7.2477 e+00$

* elev *

* idrod *

* idrodo*

* $\operatorname{rdx}$ *

* radrd *

* matrd * $f$

$-5.3528 e+00$

0.6096

$0.3198 \mathrm{e}$

$-6.5720 e+00 \quad-5.9624 e+00 s$

$-4.7432 e+00 \quad-4.2785 e+00 e$

* nfax * $\mathrm{f}$

* rftn * $\mathrm{f}$

1 e

$4.4326 e+020$

1. $2303 e-02$

$1.2513 e-02 \quad 1.2723 e-02 e$

$52 e$

$1 \mathrm{e}$

$3.7300 e+02 e$

* Upflow (Heated Pb/Al structure connected to Water only)

$\star * * \star * * *$ tyape

rod

* nopowr

0

idbci

nrods

1

atxht (1)

1. $0000 e+03$

irpwty

irpwtr

104

izpwtr

nmwrx

0

nzpwz

16

$$
\begin{array}{rr}
\text { num } & \text { id } \\
962 & 962 \\
\text { ncrz } & \text { ittc } \\
6 & 0 \\
\text { nridr } & \text { modez } \\
1 & 1 \\
\text { idbco } & \text { hdri } \\
2 & 0.0000 e+00 \\
\text { nodes } & \text { irftr } \\
6 & 0 \\
\text { dtxht (2) } & \text { dznint } \\
\text { 1.0000e+03 } & 1.0000 e+02 \\
\text { ndgx } & \text { ndhx } \\
0 & 0 \\
\text { irpwsv } & \text { nrpwtb } \\
1 & 20 \\
\text { izpwsv } & \text { nzpwtb } \\
1 & 1 \\
\text { nfci } & \text { nfcil } \\
0 & 0 \\
\text { nzpwi } & \text { nfbpwt } \\
-1 & 0
\end{array}
$$

id ctitle

962 \$962\$ Blanket row1 htr liqlev
0
haro
$2.1164 \mathrm{e}-02$
nzmax
26
hgapo

nrts

10

nrpwsv

nzpwsv

nzpwsv
0
1. $0000 \mathrm{e}+03$
$0.0000 e+00$

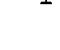

1




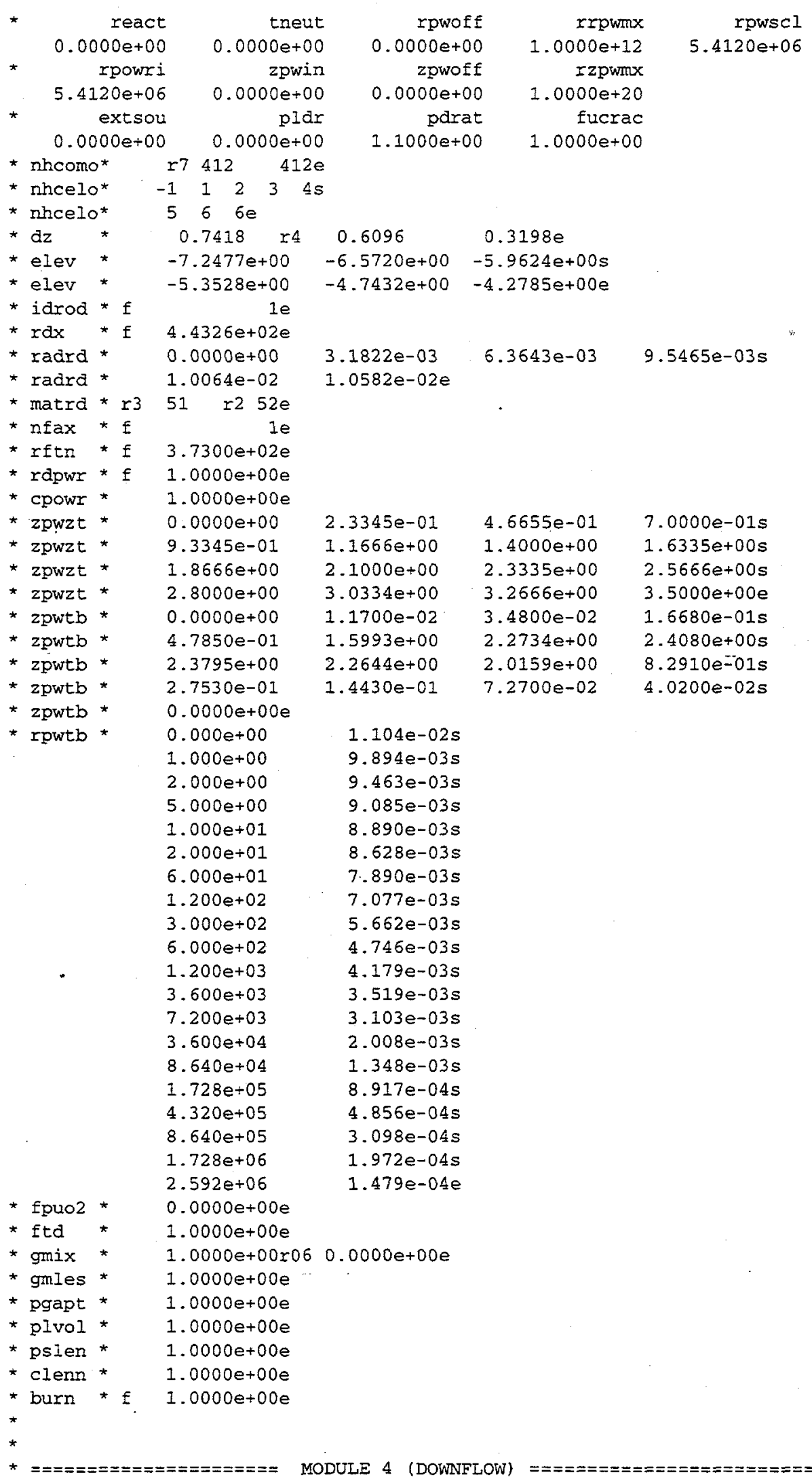


*

$$
\text { ** }
$$
rod type

num

931

nerz

* norx

* nopowr

idbci

2

nrods

1

dtxht (1)

$1.0000 e+03$

* nhcomi*

* nhceli*

* nhceli*

* nhcomo*

* Incelo*

* Incelo*

* az *

* elev *

* elev *

* idrod *

* idrodo*

* rax *

* radrd *

* matra * $f$

* nfax * $f$

* retr *

* Downflow (Heated $\mathrm{Pb} / \mathrm{Al}$ structure connected to Water only)

$\star \star \star \star \star \star * *$ type

rod

* ncrx

* nopowr

$i d b c i$

0

nrods

atxht (1)

$1.0000 e+03$

irpwty

7

104

izpwtr

nmwrx

0

nzpwz

react

$0.0000 e+00$

rpowri

$3.9240 e+06$

extsou

$0.0000 e+00$

* nhcomo*

$r 7480$

nriar

idbco

2

nodes

dtxht (2)

$1.0000 e+03$

r7 480

$480 e$

$566 e$

17823

823 e

$\begin{array}{llllll}-7 & 7 & 6 & 5 & 4\end{array}$

$322 e$

$0.3198=4$

$-4.2785 e+00$

0.6096

$0.7418 \mathrm{e}$

$-5.9624 e+00$

$-4.7432 e+00$

$-5.3528 \mathrm{e}+00 \mathrm{~s}$

id

ctitle

itto

0e-02

0

dznht

$1.0000 e+02$

1.0000

$5.3477 e-02$

nzmax

hgapo

2.8076

$8076 e+02 e$

$1.6882 e-02 \quad 1.7076 e-02 \quad 1.7269 e-02 e$

$52 \mathrm{e}$

$1 e$

$3.7300 e+02 e$

$-6.5720 e+00 \quad-7.2477 e+00 e$

num id ctitle

978

ittc

6

nridr

idbco

nodes

dtxht (2)

$1.0000 e+03$

ndgx

irpwsv

1

izpwsv

nfci

0

nzpwi

$-1$

$0.0000 e+00$

zpwin

$0.0000 e+00$

plar

$0.0000 e+00$

$480 \mathrm{e}$

\section{$0.0000 e+00$}

irftr

dznint

$1.0000 e+02$

ndh $x$

nrowtb

20

nzpwtb

1

nfcil

nfbpwt

rpwof

$0.0000 e+00$

zpwoff

$0.0000 e+00$

parat

$1.1000 e+00$

rzpwmx

$1.0000 e+20$

fucrac

$1.0000 e+00$

931 \$931\$He to Aluminum and then water

shelv

$0.0000 e+00$

axcnd

978 \$978\$ Blanket row2 htr

rrpwinx

$1.0000 e+12$

iaxend

2.1164 e-02

nzmax

hgapo

$1.0000 e+03$

nrts

nrpwsv

nzpwsv

0

0.0000

$0 \mathrm{e}+00$

nhist

nxpwrf

nzpwrf

0

3. $9240 e+06$ 
* nhcelo*

* nhcelo*

$\star d z$ *

* elev *

* elev *

* idrod * $f$

$* r \mathrm{dx} * \mathrm{E}$

* radra *

* radra *

* matra * r3

* nfax * $\mathrm{f}$

* retr *f

* rdpwr * $f$

* cpowr *

* zpwzt *

* zpwzt *

* zpwzt *

* zpwzt *

* zpwtb *

* zpwtb *

* zpwtb *

* zpwtb *

* rpwtb *
* zpwtb *

$\begin{array}{lllll}-1 & 1 & 2 & 3 & 4\end{array}$

$566 \mathrm{e}$ $0.3198 \quad r 4$

$-4.2785 e+00$

$-5.9624 e+00$

1

$.8076 e+02 e$

$0.0000 \mathrm{e}+00$

$1.5497 e-02$

$51 \times 252 e$

$3.7300 e+02 e$

$1.0000 \mathrm{e}+00 \mathrm{e}$

$1.0000 \mathrm{e}+00 \mathrm{e}$

$0.0000 \mathrm{e}+00$

$9.3345 \mathrm{e}-01$

$1.8666 e+00$

$2.8000 e \div 00$

$0.0000 \mathrm{e}+00$

$4.7850 \mathrm{e}-01$

$2.3795 e+00$

$2.7530 e-01$

$0.0000 \mathrm{e}+00 \mathrm{e}$

$0.000 e+00$

$1.000 e+00$

$2.000 e+00$

$5.000 e+00$

$1.000 e+01$

$2.000 e+01$

$6.000 e+01$

$1.200 e+02$

$3.000 e+02$

$6.000 e+02$

1. $200 e+03$

$3.600 e+03$

$7.200 e+03$

$3.600 e+04$

8. $640 e+04$

1. $728 e+05$

4. $320 e+05$

$8.640 e+05$

1. $728 e+06$

$2.592 \mathrm{e}+06$

* fpuo2 *

$0.0000 \mathrm{e}+00 \mathrm{e}$

* ftd

$1.0000 \mathrm{e}+00 \mathrm{e}$

* gmix *

* gmles *

* pgapt *

* plvol *

* pslen *

* clenn

* burn *

$1.0000 e+00 e$

$1.0000 e+00 e$

$1.0000 \mathrm{e}+00 \mathrm{e}$

$1.0000 e+00 e$

$1.0000 \mathrm{e}+00 \mathrm{e}$

$1.0000 \mathrm{e}+00 \mathrm{e}$

$2.3345 e-01$

$1.1666 e+00$

$4.6655 e-01$

$7.0000 e-01 \mathrm{~s}$

2. $1000 e+00$

$3.0334 \mathrm{e}+00$

1. $1700 \mathrm{e}-02$

$1.5993 \mathrm{e}+00$

$2.2644 e+00$

1. $4430 e-01$

$1.4000 \mathrm{e}+00$

$2.3335 e+00$

$3.2666 \mathrm{e}+00$

$1.6335 \mathrm{e}+00 \mathrm{~s}$

3. $4800 \mathrm{e}-02$

$5666 \mathrm{e}+00 \mathrm{~s}$

$2.4800 e-02$

$3.5000 \mathrm{e}+00 \mathrm{e}$

$2.0159 e+00$

1. $6680 \mathrm{e}-01 \mathrm{~s}$

$2.4080 \mathrm{e}+00 \mathrm{~s}$

$7.2700 e-02$

8.2910e-01s

$4.0200 \mathrm{e}-02 \mathrm{~s}$

1. $402 \mathrm{e}-02 \mathrm{~s}$

$1.220 \mathrm{e}-02 \mathrm{~s}$

$1.146 e-02 \mathrm{~s}$

$1.089 \mathrm{e}-02 \mathrm{~s}$

$1.068 \mathrm{e}-02 \mathrm{~s}$

$1.041 e-02 s$

$9.584 \mathrm{e}-03 \mathrm{~s}$

$8.659 \mathrm{e}-03 \mathrm{~s}$

$7.036 e-03 s$

$5.983 e-03 s$

$5.332 e-03 \mathrm{~s}$

$4.543 e-03 s$

$4.012 e-03 s$

$2.586 \mathrm{e}-03 \mathrm{~s}$

$1.747 \mathrm{e}-03 \mathrm{~s}$

$1.168 \mathrm{e}-03 \mathrm{~s}$

$6.158 \mathrm{e}-04 \mathrm{~s}$

$3.734 e-04 \mathrm{~s}$

$2.259 \mathrm{e}-04 \mathrm{~s}$

$1.625 \mathrm{e}-04 \mathrm{e}$

$0.0000 e+00 e$

*

MODULE 5 (UPFLOW)

* Upflow (Unheated Al structure connected to Water \& Cavity)

$* \star \star \star \star \star \star *$ type num

rod

* norx

1

* nopowr nridr

913

ncrz

6

nridr

m id

$913 \$ 913 \$$ He to Aluminum and then water ittc

modez

Iiqlev

iaxend 
Report:

Section:

Date:

Page:

shelv

$0.0000 e+00$

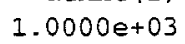


* radrd * $1.5497 e-02$

* matrd * r3 51 r2 $52 \mathrm{e}$

* nfax * $\mathrm{f}$ le

* rftn *f $3.7300 e+02 e$

* rapwr *f $1.0000 e+00 \mathrm{e}$

* cpowr * $1.0000 e+00 e$

* zpwzt * $0.0000 e+00$

* zpwzt * $9.3345 e-01$

* zpwzt * $1.8666 \mathrm{e}+00$

* zpwzt * 2.8000e+00

* zpwtb * $0.0000 e+00$

* zpwtb * 4.7850e-0I

* zpwtb * $2.3795 e+00$

* zpwtb * 2.7530e-01

* zpwtb * $0.0000 e+00 e$

* rpwtb * $0.000 e+00$

$1.000 e+00$

$2.000 e+00$

$5.000 e+00$

$1.000 e+01$

$2.000 e+01$

$6.000 e+01$

1. $200 \mathrm{e}+02$

$3.000 e+02$

$6.000 e+02$

$1.200 \mathrm{e}+03$

$3.600 \mathrm{e}+03$

$7.200 e+03$

$3.600 e+04$

$8.640 e+04$

$1.728 \mathrm{e}+05$

$4.320 e+05$

$8.640 e+05$

1. $728 e+06$

$2.592 e+05$

* fpuo2 *

$0.0000 e+00 e$

* Etd * $1.0000 e+00 e$

* gmix * $1.0000 e+00 r 060.0000 e+00 e$

* gmles * $1.0000 e+00 \mathrm{e}$

* pgapt * $1.0000 e+00 \mathrm{e}$

* plvol * 1.0000e+00e

* pslen * 1.0000e+00e

* clenn * $1.0000 e+00 e$

* burr * E $1.0000 e+00 e$
$5.0017 e-03$

$1.5989 \mathrm{e}-02 \mathrm{e}$

$2.3345 e-01$

$1.1666 \mathrm{e} \div 00$

$2.1000 e+00$

$3.0334 \mathrm{e}+00$

$1.1700 \mathrm{e}-02$

$1.5993 e+00$

$2.2644 e+00$

$1.4430 e-01$

$1.220 \mathrm{e}-02 \mathrm{~s}$

$1.146 \mathrm{e}-02 \mathrm{~s}$

$1.089 e-02 \mathrm{~s}$

$1.068 \mathrm{e}-02 \mathrm{~s}$

$1.041 \mathrm{e}-02 \mathrm{~s}$

$9.584 e-03 s$

$8.659 e-03 s$

$7.036 e-03 s$

$5.983 e-03 s$

$5.332 \mathrm{e}-03 \mathrm{~s}$

$4.543 \mathrm{e}-03 \mathrm{~s}$

$4.012 \mathrm{e}-03 \mathrm{~s}$

$2.586 \mathrm{e}-03 \mathrm{~s}$

$1.747 e-0.3 \mathrm{~s}$

$1.168 \mathrm{e}-03 \mathrm{~s}$

$6.158 \mathrm{e}-04 \mathrm{~s}$

$3.734 \mathrm{e}-04 \mathrm{~s}$

$2.259 e-04 \mathrm{~s}$

I. $625 \mathrm{e}-04 \mathrm{e}$
$1.402 \mathrm{e}-02 \mathrm{~s}$
$1.0003 e-02$

$1.5005 \mathrm{e}-02 \mathrm{~s}$

\section{4. $6655 e-01$}

1. $4000 e+00$

$2.3335 e+00$

$3.2666 e+00$

$3.4800 e-02$

$2.2734 e+00$

$2.0159 e+00$

$7.2700 e-02$

$7.0000 \mathrm{e}-01 \mathrm{~s}$

$1.6335 \mathrm{e}+00 \mathrm{~s}$

$2.5666 \mathrm{e}+00 \mathrm{~s}$

$3.5000 \mathrm{e}+00 \mathrm{e}$

$1.6680 \mathrm{e}-01 \mathrm{~s}$

$2.4080 \mathrm{e}+00 \mathrm{~s}$

$8.2910 \mathrm{e}-01 \mathrm{~s}$

4. $0200 \mathrm{e}-02 \mathrm{~s}$

*

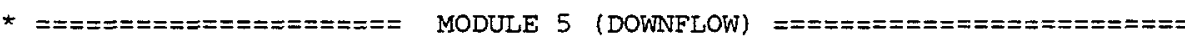

* Downflow (Unheated Al structure connected to Water \& Cavity)

$* * \star * * * * *$ type num

$\operatorname{rod} 932$

* nerx ncrz

$\begin{array}{rrr}\text { * ncrx } & \text { ncrz } \\ * & 1 & 6\end{array}$

* nopowr $\quad$ nriar

idbci idbco

2

nrods

1

atxht (1)

nodes

dexht (2)

1. $0000 \mathrm{e}+03$

1. $0000 e+03$

$$
\begin{array}{r}
\text { id } \\
932 \\
\text { ittc }
\end{array}
$$

$932 \$ 932 \$$ He to Aluminum and then water

0
modez
1
hari
1.0210 e-02
irftr
0
dznht

1. $0000 e+02$

$$
\begin{array}{r}
\text { liqlev } \\
0 \\
\text { haro } \\
5.3477 e-02 \\
\text { nzmax } \\
23 \\
\text { hgapo }
\end{array}
$$$$
1.0000 \mathrm{e}+03
$$

shelv

iaxcnd$$
1
$$

$0.0000 e+00$ 


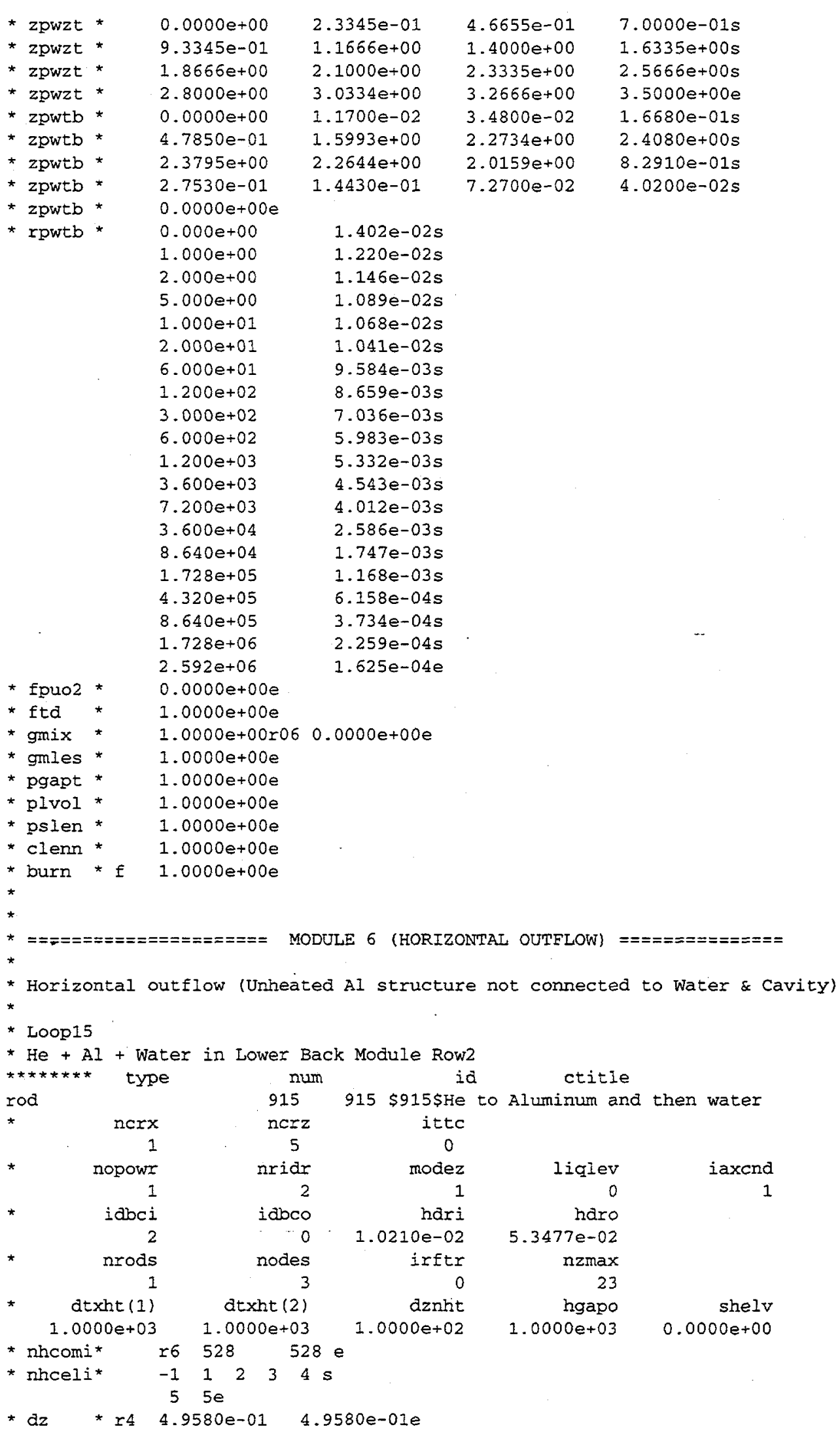


* elev * $\quad-7.1317 \mathrm{e}+00 \quad-7.1317 \mathrm{e}+00 \quad-7.1317 \mathrm{e}+00 \mathrm{~s}$

* elev *

* idrod *

$-7.1317 e+00 \quad-7.1317 e+00 e$

* idrodo*

* $r d x$

* radrd*

$1.3224 \mathrm{e}+02 \mathrm{e}$

* matra * $f$

$\star \operatorname{nfax} * \mathrm{E}$

$1.6882 \mathrm{e}-02$

$1.7076 e-02$

$1.7269 e-02 e$

$* \operatorname{rftn} * f$

$52 e$

$3.73000+020$

* Horizontal outflow (Heated Pb/Al structure connected to water only)

* Loop 15

* Lower Blanket Back Module Row2 Region

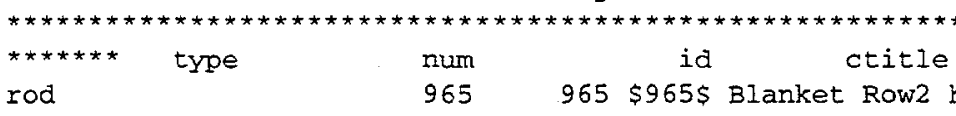

nerz

nerx

nopowr

icbci

nrods

1

dtxht (1)

$1.0000 \hat{e}+03$

irpwty

irpwtr

104

izpwtr

nmwrx

0

nzpwz

react

$0.0000 €+00$

rpowri

$5.7120 e+06$

extsou

$0.0000 e+00$

* nhcomo*

* nhcelo*

$$
\text { r6 } 528
$$

$\begin{array}{ll}-1 & 1\end{array}$

* $\mathrm{d} z$

$55 e$

* elev *

* elev *

* iarod * $f$

$\star r d x * f$

* radra *

* radra *

* matrd * r3

* nfax *

* rftn *

* rdpwr * $f$

* cpowr *

* zpwzt *

* zpwzt *

* zpwtb *

4.9580 e-01

$-7.1317 e+00$

$-7.1317 \mathrm{e}+00$

$1 e$

$1.3224 e+02 e$

$0.0000 e+00$

$1.5497 \mathrm{e}-02$

$51 \quad r 252 \mathrm{e}$

$1.0000 e+00 e$

$1.0000 \mathrm{e}+00 \mathrm{e}$

$0.0000 \mathrm{e}+00$

1. $9832 \mathrm{e}+00$
$2.4790 e+00 e$

$1.0003 e-02$

$1.5005 e-02 \mathrm{~s}$

1.0000 et03

nrts

nrpwsy

nzpwsv

0

rrpwinx

rpwsc1

$0.0000 e+00$

zpwoff

$0.0000 e+00$

pdrat

$1.1000 e+00$

1. $0000 e+12$

rzpwinx

$1.0000 e+20$

fucrac

$1.0000 e+00$

4.9580 e-01e

$-7.1317 e+00 \quad-7.1317 e+00 s$

$-7.1317 \mathrm{e}+00 \mathrm{e}$

$0.0000 e+00$

nhist

nxpwrf
0
nzpwrf

nxpwrf
0
nzpwrf

$5.7120 e+06$

0

\section{a}

$1164 e-02$

26

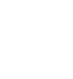

$1.0000 e+00 \quad 0.0000 e+00 e$ 


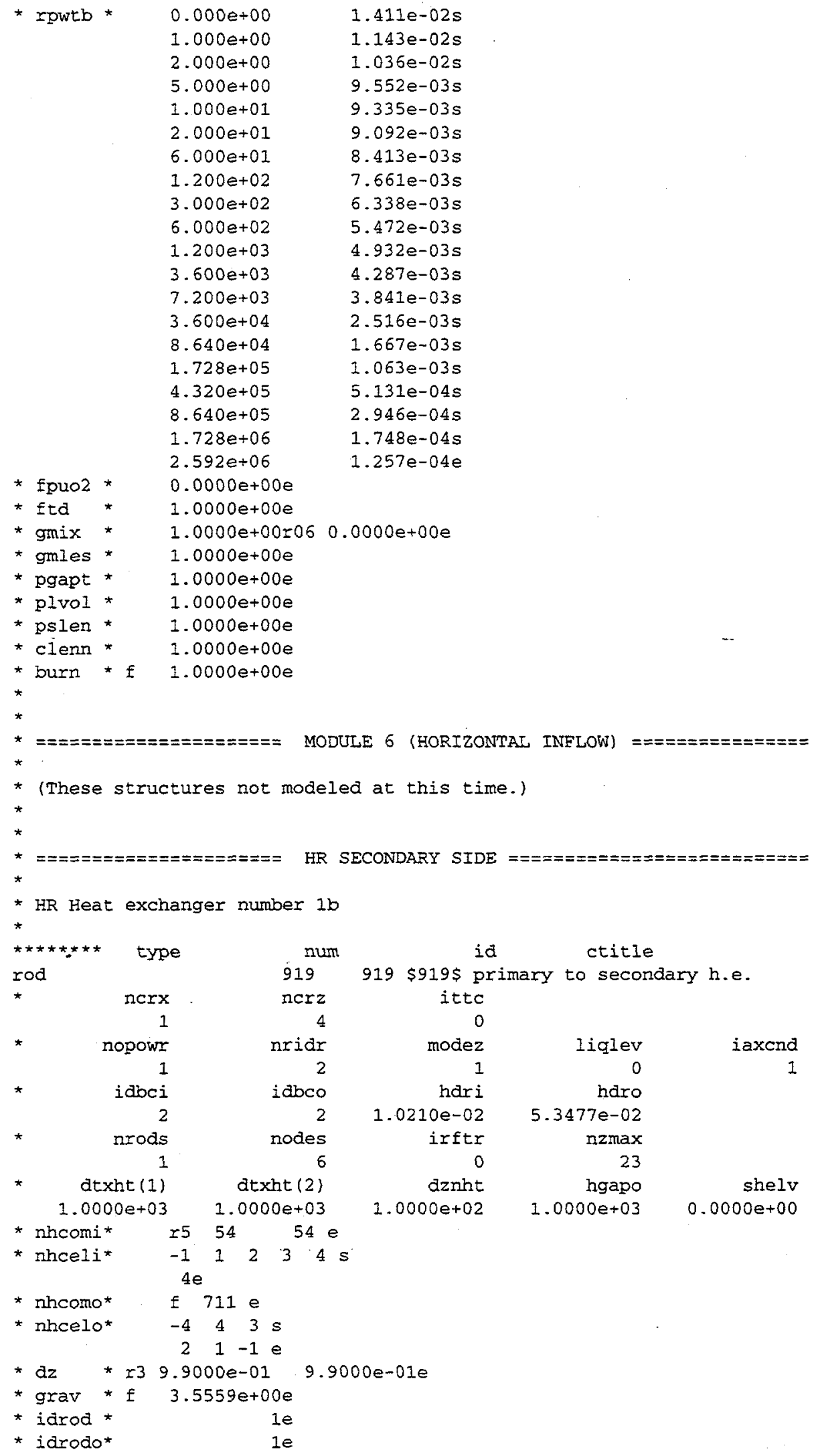


* $r a x$

* radrd *

* radra *

* matrd * $f$

$* \operatorname{nfax} * f$

* rftn * $f$

*

* HR Heat exchanger number Ia

$* * * * * * * *$ type

rod

$6.9080 e+03 e$

$5.1050 e-03$

$6.1010 e-03$

5. $3540 e-03$

$6.3500 \mathrm{e}-03 \mathrm{e}$

$5.6030 e-03$

$5.8520 e-03 s$

$3.780 e+02 e$

id

ctitie

$920920 \$ 920 \$$ primary to secondary h.e.

$$
\text { nerx }
$$

1

nopowr

idbci

2

nrods

1

$\operatorname{dtxht}(1)$

$1.0000 e+03$

* nhcomi*

* nhceli*

* nhcomo*

* nhcelo*

* $d z$

* idrod *

* idrodo*

* rax *

* radra *

* radra *

* matra *

* nfax * $f$

$\star \operatorname{rftn} * \pm$

* HR Heat exchanger number 2b

$\star \star \star \star \star * * *$ type

$\operatorname{rod}$

*

$$
\begin{array}{r}
\text { nerx } \\
1
\end{array}
$$

nopowr

$$
\text { idbci }
$$

$$
\text { nrods }
$$

1

atxht (1)

* nhcomi *

* nhceli*

$$
r 5 \quad 55
$$

$6.9080 e+03 e$

5.1050 e-03

$6.1010 \mathrm{e}-03$

$5.3540 e-03$

$6.3500 e-03 e$

$5.6030 \mathrm{e}-03$

$5.8520 e^{-03 s}$

1.0210e-02

irftr

dznht

1. $0000 \mathrm{e}+02$

ncrz

nridr

idbco

lodes

atxht (2)

50

7 e

$3.780 e+02 e$

$9.9000 e-01 e$

$1 e$

* nhcomo*

* nucelo*

$\begin{array}{llll}-1 & 1 & 2\end{array}$

$4 \mathrm{e}$

f $731 \mathrm{e}$

-443 s

* +23 2 $1-1$

* grav * f $3.5559 \mathrm{e}+00 \mathrm{e}$

\section{num. \\ 921}

ncrz

nridr

iabco

nodes

dexht (2)

$0000 e+03$

55 e

$4 \mathrm{~s}$

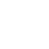

$9.9000 e-01 e$

id ctitle

921 \$921\$ primary to secondary h.e.

ittc

odez

1

liqlev

iaxcond

shelv

$1.0000 e+03 \quad 0.0000 e+00$

iaxcnd

\section{1}

$.3477 e-02$

nzmax

hapo

0.0000 eto 
* idrod * 10

* idrodo*

$* r \mathrm{dx} *$

$6.9080 e+03 e$

* radrd *

$5.1050 e-03$

$6.1010 e-03$

$5: 3540 e-03$

$5.6030 e-03$

$5.8520 \mathrm{e}-03 \mathrm{~s}$

* radrd * $6.3500 \mathrm{e}-03 \mathrm{e}$

* matra *

* nfax * $\mathrm{f}$

$* \operatorname{rftn} * f$

*

*

* HR Heat exchanger number 2a

$\star * \star * * * * *$ type

rod

num

id ctitle

* nor $\quad$ x

* ropowr

$\begin{array}{rr}* & \text { nopowr } \\ * & 1 \\ & \text { idbci }\end{array}$

* $\quad$ idbci

* nrods

$1 e$

le

$3.780 e+02 e$

95 of 96

* atxht (1)

$1.0000 e+03$

dtexht

922

922 \$922\$ primary to secondary h.e.

nerz ittc

ahcomi* r5 51

* nhceli* $-1 \quad 12334$

* nhcomo* f $733^{\circ}$ e

* nhcelo* $-4 \quad 435$

$21-1 e$

*dz * r3 9.9000e-01

* grav *f $5.0559 e+00 e$

* idrod *

* idrodo*

$* r d x$ *

* radra *

$5.1050 e-03$

$5.3540 e-03$

$5.6030 e-03$

$5.8520 e-03 s$

* matra * $f$

$* \operatorname{nfax} * f$

$6.3500 e-03 e$

modez

$1.0210 e-02$

5. $3477 e-02$

irftr

nzmax

$1.0000 e+02$

hgapo

$1.0000 e+03$

iaxend

* rftr $\star f$

$3.780 e+02 e$

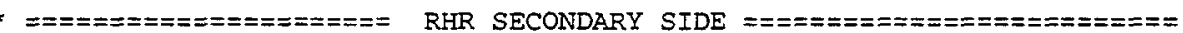

* RHR Heat exchanger

$\star * \star * * * * *$ type

$\operatorname{rod}$

num id ctitle

* norx

971

971 \$971\$ rhr to secondary h.e.

* nerx

* nopowr

norz

1

idbci

2

* $\quad$ nrods

atxht (1)

$1.0000 \mathrm{e}+03$

* nhcomi* r5 652

$0000 e+03$

* ninceli*

$-1$

* nicomo*

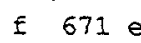

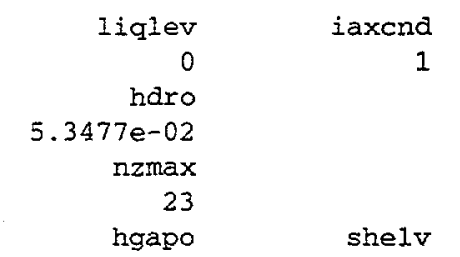

1. $0000 e+03$

$0.0000 e+00$
$1.00000+02$

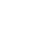


* nhcelo* $\quad \begin{array}{rrrr}-4 & 4 & 3 & s \\ 2 & 1 & -1 & e\end{array}$

* dz *r39.9000e-01 9.9000e-01e

* grav * e $2.9560 e+00 e$

* idrod * le

* idrodo* $1 e$

* rdx * $2.7700 \mathrm{e}+02 \mathrm{e}$

* raara * 5.1050 e-03

* radra * 6.1010e-03

$$
5.3540 e-03
$$

$6.3500 e-03 e$

* matrd * $f$

* nfax * $7 e$

* rftn * $\pm 3.780 e+02 e$

*

end

$\star$

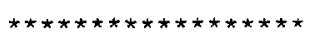

* time-step data *

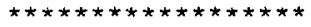

$\star$

* atmin

1.0000e-04

* edint

dtmax

1. 0000 e-00

gfint

tend

4. $0000 e+02$

ampint

$1.0000 e+01$

$5.0000 e+00$

1. $0000 e+06$

rtwfp
$1.0000 e+01$

sedint

* endflag

$-1.0000 e+00$ 


\section{Appendix B: TRAC Graphics Input File for Normal Operation Case}

The file listed below represents the entire TRAC code "graphin" file that contains the various graphics points selected for output to the "tecsum.grf" file. Modifications were made to the TRAC code standard output routines in order to generate an ASCll output file called "tecsum.grf". This output file contains results at each requested graphic point (i.e., TRAC component and specified cell number) written out at a specified time frequency.

In a post-processing mode, the graphic ASCII output file is split into individual ASCII files for each graphic point using a special purpose fortran program named "TSPLIT". The output from TSPLIT was formatted to be TecPlot ${ }^{\mathrm{TM}}$ ready. TecPlot ${ }^{\mathrm{TM}}$ is a widely used commercial scientific and engineering data visualization software package for creating multi-dimensional graphics interactively. For additional details regarding the post-processing procedure see Ref. [7].

\section{Input file graphin:}

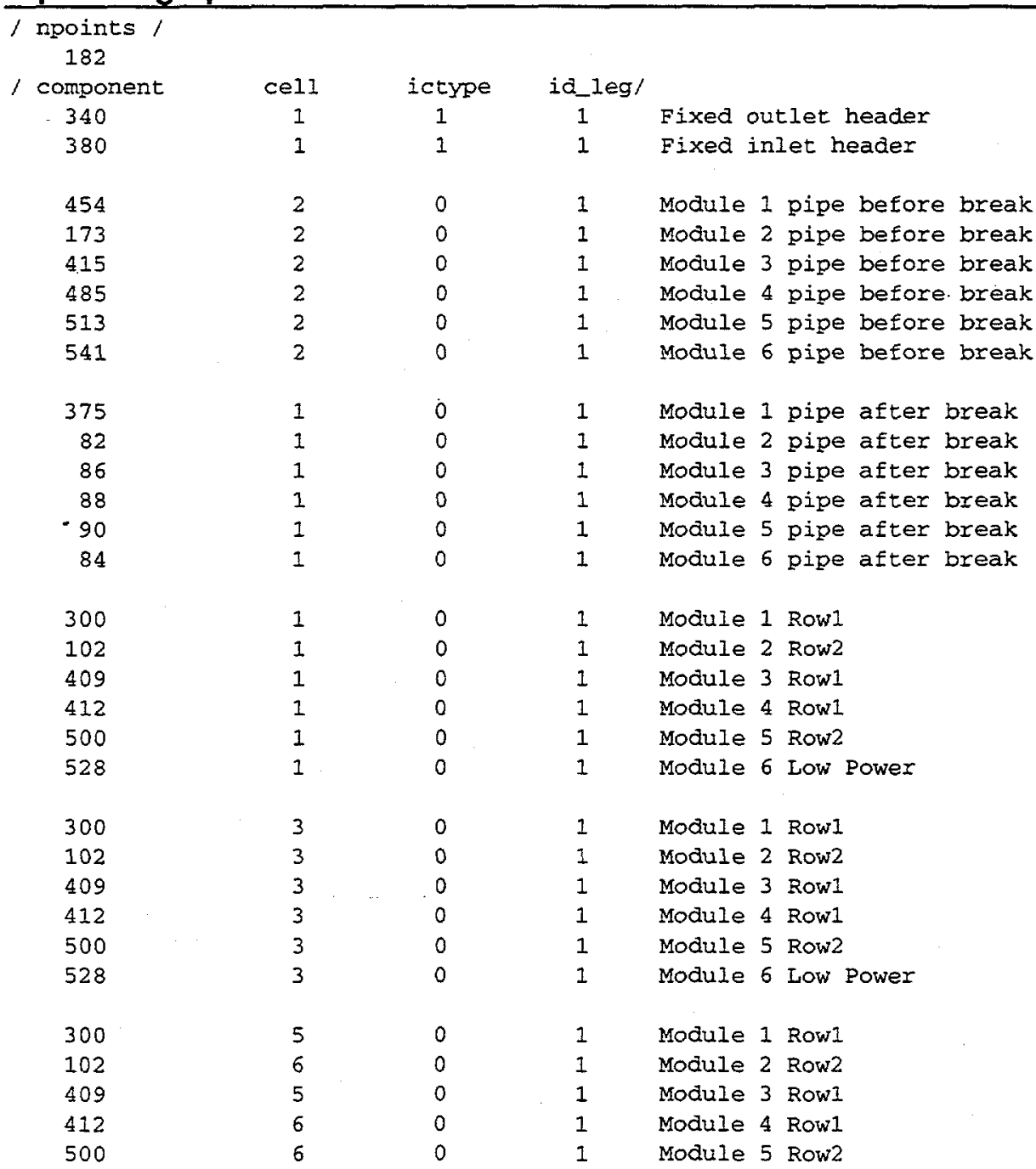




528

370

350

330

172

147

133

479

418

423

489

419

483

510

503

508

538

531

536

26

22

20

30

30

31

31

32

33

37

48

52

56

49

53

57

713

710

733

730

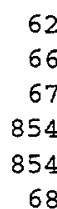

630

630

640

660

671

672

761

68

5

1

1

1

1

1

1

1

I

1

1

1

1

1

1
1

1

1

1

1

1

7

1

1

2

1

2

2

2

2

3

4

1
4

4
1

1

1

1

1

2

0

0

1 Module 6 Low Power

1

1

1

1

1

1

1

0

\begin{abstract}
0
\end{abstract}

$\begin{array}{ll}0 & 1 \\ 0 & 1 \\ 1 & 1 \\ 0 & 1 \\ 0 & 1 \\ 0 & 1\end{array}$

$\begin{array}{ll}0 & 1 \\ 0 & 1 \\ 0 & 1 \\ 0 & 1\end{array}$

71

0
0

0

Module 1 Inlet Plenum Module 1 Middle Plenum Module 1 outlet Plenum Module 2 Inlet Plenum Module 2 middle Plenum Module 2 outiet Plenum Module 3 Inlet Plenum Module 3 Middle Plenum Module 3 outlet Plenum Module 4 Inlet Plenum Module 4 Midale Plenum Module 4 Outlet Plenum Module 5 Inlet Plenum Module 5 Middle Plenum Module 5 outlet Plenum Module 6 Inlet Plenum Module 6 Middle Plenum Module 6 outlet Plenum

Hot leg pump suction line

Hot leg pump suction line near break Hot leg pump suction line near break

PCL Pump I Suction

PCL Pump 1 Discharge

PCI Pump 2 Suction

PCL Pump 2 Discharge

PCL Pump 1 check valve

PCL Pump 2 check valve

PCL Pump to $\mathrm{HX}$

PCL HX 1 inlet

PCI $\mathrm{Hx}$ I middle

PCL $\mathrm{Hx} 1$ outlet

PCL HX 2 inlet

PCI $\mathrm{Hx} 2$ middle

PCL $\mathrm{HX} 2$ outlet

PCL HX 1 secondary side outlet

PCL $\mathrm{HX} 1$ secondary side inlet fill

PCL HX 2 secondary side outlet

PCL HX 2 seconcary side inlet fill

Cold leg $\mathrm{Hx}$ discharge line

Cold leg $\mathrm{Hx}$ discharge line near break Cold leg Hx discharge line near break Cold leg $\mathrm{Hx}$ discharge line near break Cold leg $\mathrm{Hx}$ discharge line near break Cold leg $\mathrm{Hx}$ discharge line near break

RHR Pump Suction

RHR Purnp Discharge

RHR $\mathrm{Hx}$ inlet

RHR Hx outlet

RHR $\mathrm{Hx}$ secondary side outlet

RHR HX secondary side inlet fill

1

Pzr Pressure Signal 


\begin{abstract}
Pzr Bottom Pressure
\end{abstract}
Hot Module (I) upflow inside Hot Module (1) upflow inside Hot Module (1) upflow inside Hot Module (1) upflow outside Hot Module (1) upflow outside Hot Module (1) upflow outside Hot Module (2) upflow inside Hot Module (2) upflow inside Hot Module (2) upflow inside Hot Module (2) upflow outsice Hot Module (2) upflow outside Hot Module (2) upflow outside Hot Module (3) upflow inside Hot Module (3) upflow inside Hot Moduie (3) upflow outside Hot Module (3) upflow outside Hot Module (3) upflow outside Hot Module (3) upflow outside Hot Module (4) upflow inside Hot Module (4) upflow inside Hot Module (4) upflow inside Hot Module (4) upflow outside Hot Module (4) upflow outside Hot Module (4) upflow outside Hot Module (5) upflow inside Hot Module (5) upflow inside Hot Module (5) upflow inside Hot Module (5) upflow outside Hot Module (5) upflow outside Hot Module (5) upflow outside Hot Module (6) upflow inside Hot Module (6) upflow inside Hot Module (6) upflow inside Hot Module (6) upflow outside Hot Module (6) upflow outside Hot Module (6) upflow outside

Flood valve pool side

Flood valve vessel side

Elood horizontal line Flood horizontal line Flood horizontal line Flood vertical line Flood vertical line Flood vertical line

Cavity vessel lower 1 cavity vessel lower 2 Cavity vessel lower 3 Cavity vessel lower 4 Cavity vessel lower 6 Cavity vessel lower 8 Cavity vessel lower 10 Cavity vessel lower 11

Cavity line to Hot module loc. 1 Cavity line to Hot module loc. 2 Cavity vessel Hot module $10 \mathrm{c}$. Cavity vessel middle 1 


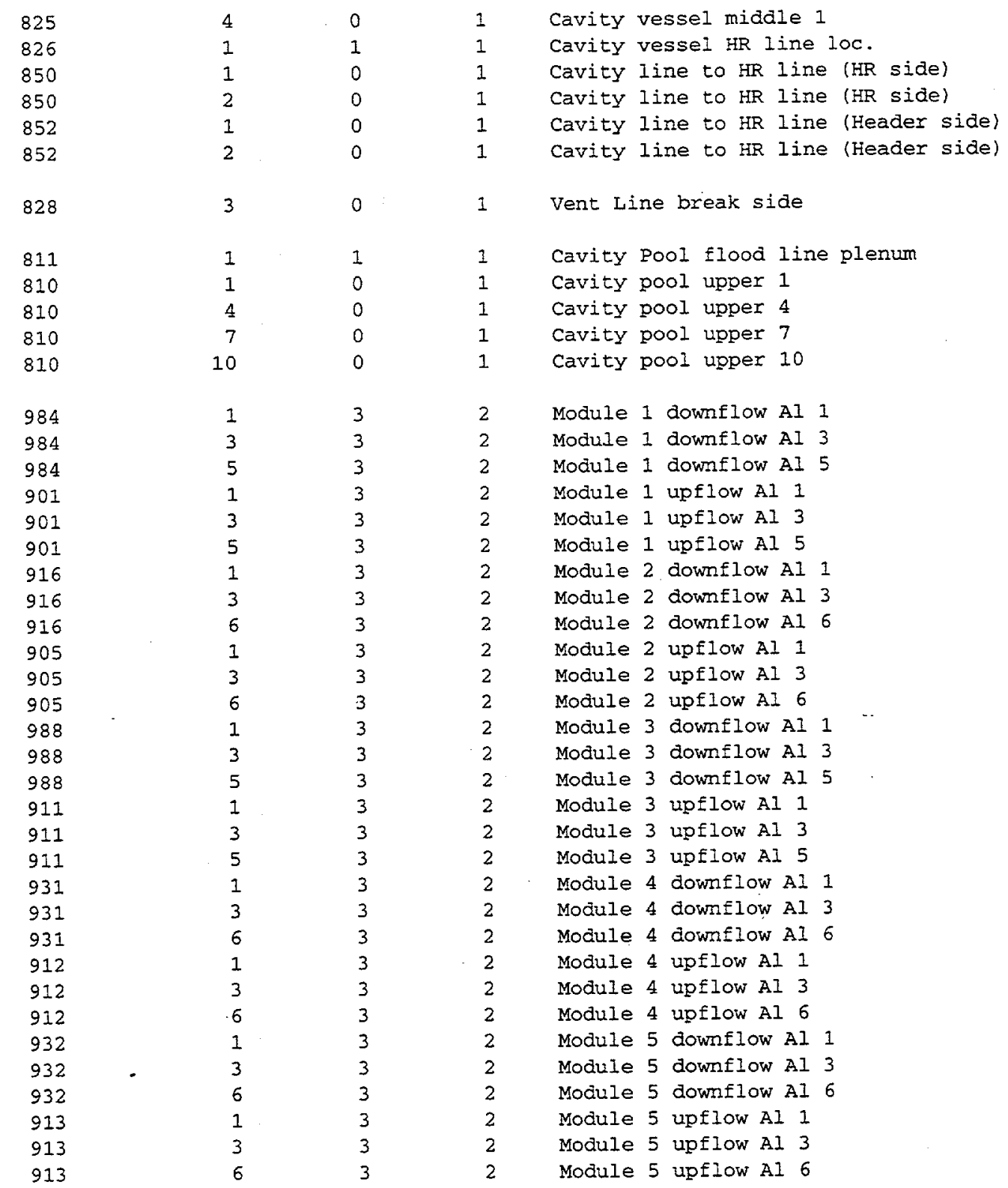

INPUT NOTES:

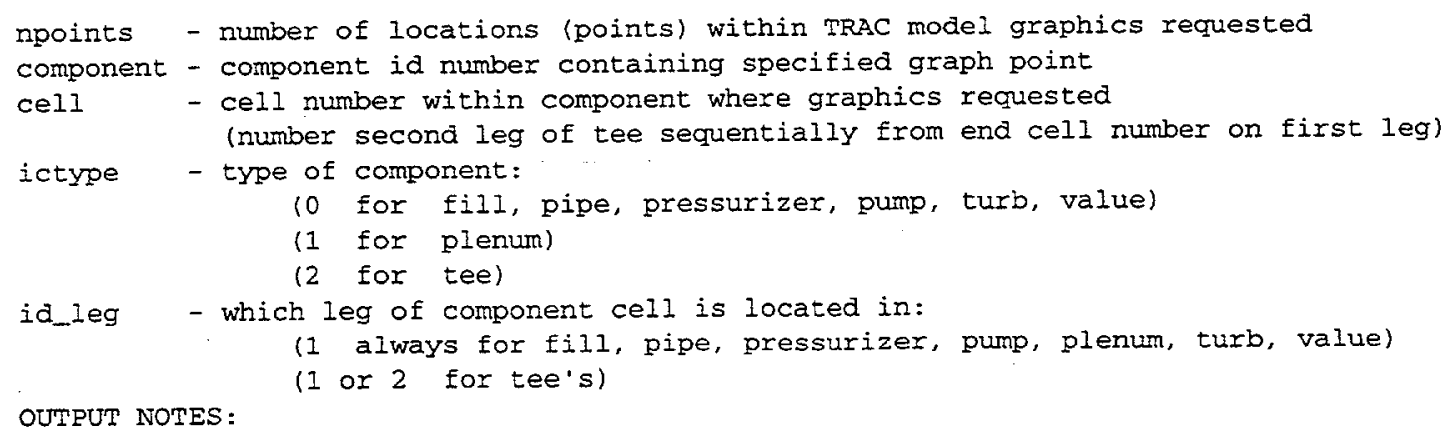




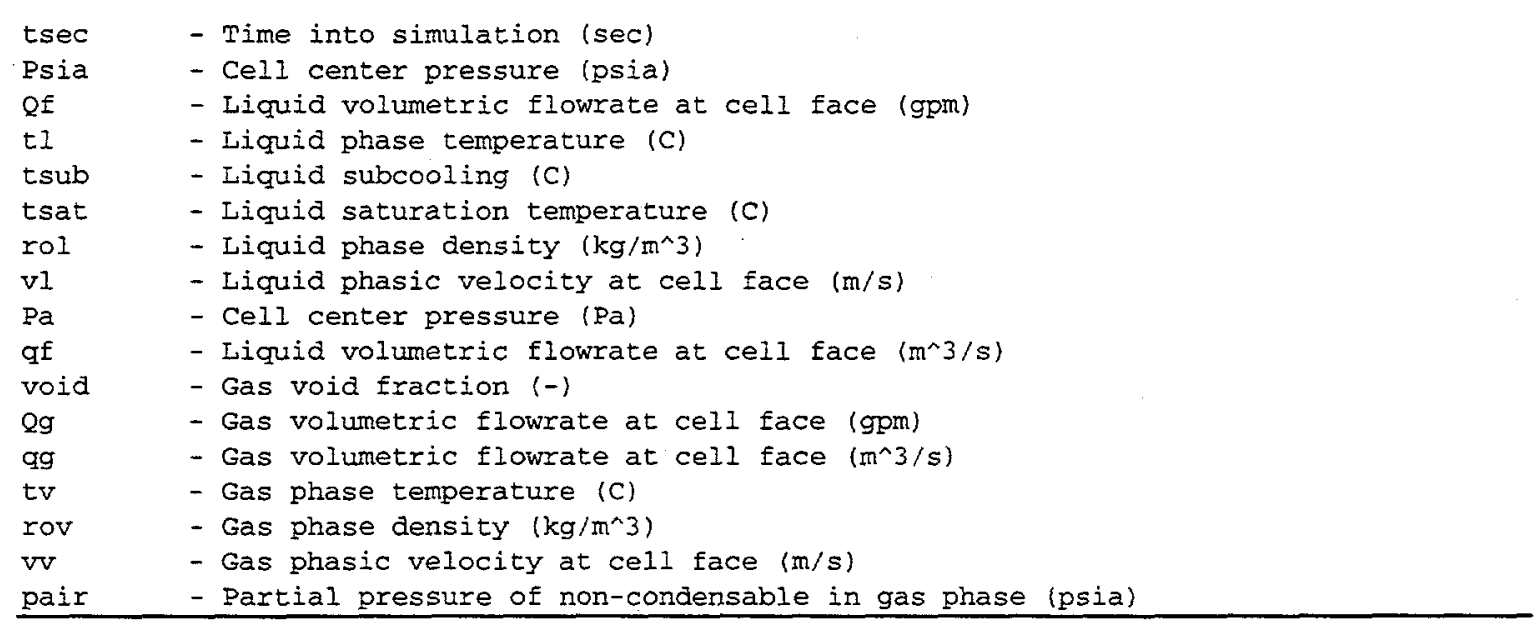


(This Page Intentionally Left Blank) 


\section{Appendix C: QKTRAC - A Simple Hydraulic Code for HR Assessment}

This appendix contains the source listing for the code named QKTRAC and all of its associated subroutines. QKTRAC was written specifically for assessing the steadystate isothermal hydraulic behavior of the HR coolant loop. Sample input and output files are provided.

\section{Program qtrac.f:}

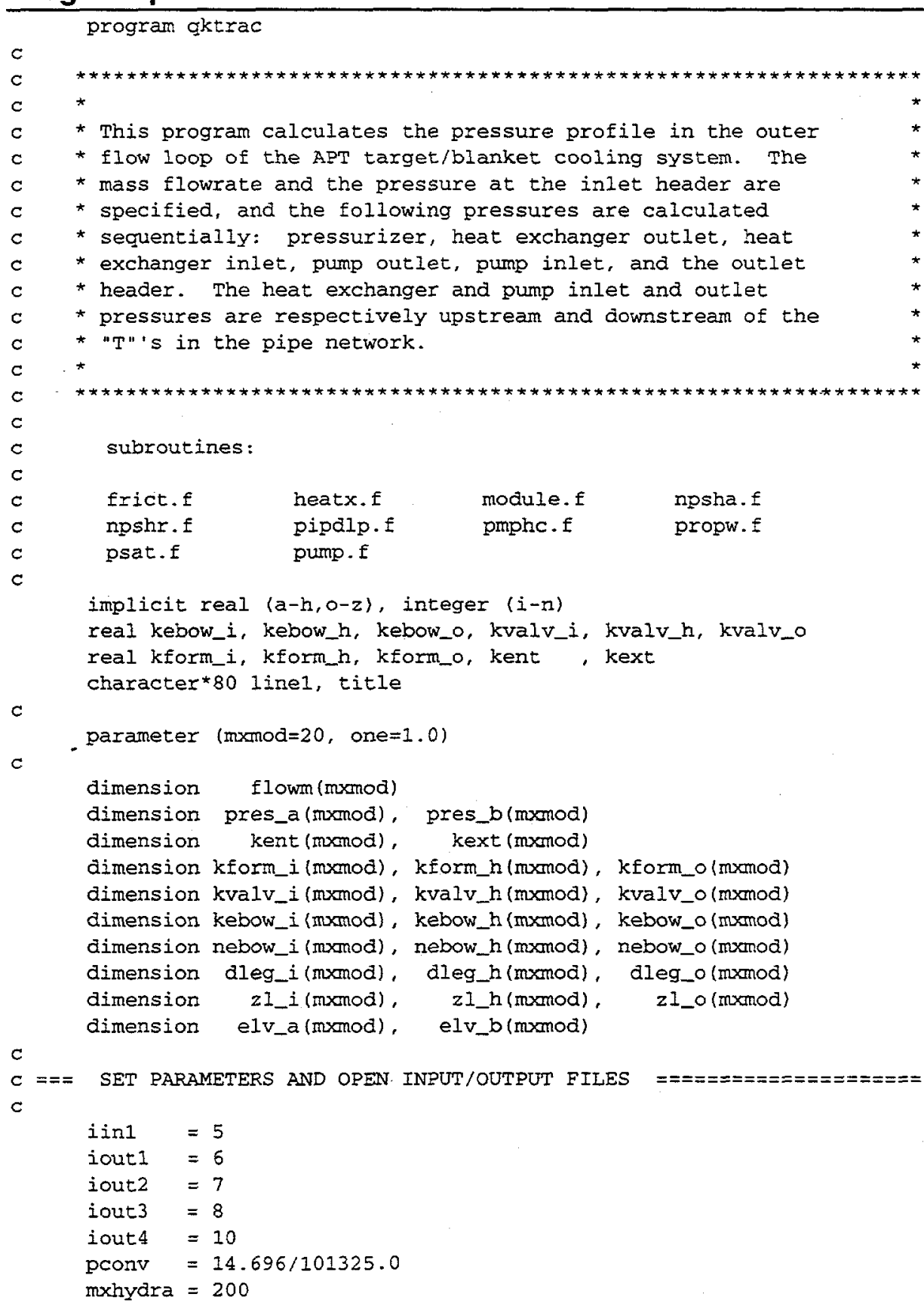


hconvg $=1.0 \mathrm{e}-2$

weight $=0.5$

c

open (unit=iinl, file='input.dat' , status='old')

open (unit=iout 1 , file='results.out', status='unknown')

open (unit=iout2, file='input. in' ,status='unknown')

open (unit=iout3, file='heatX.out' ,status='unknown')

open (unit=iout 4 , file='pumps.out' , status='unknown')

$c$

$c==$

$c$

c

c

c

c

c

c

$c$

c

c

c

c

c

$$
\text { do }
$$


endado

c

write (iout2, 2020)

read (iinl, 900) linel

write (iout2,900) linel

read (iin1,*) $z \lg 2, \operatorname{diam} 2, \operatorname{del} z 2$

write (iout2,*) $z \lg 2, \operatorname{diam} 2, \operatorname{del} 22$

reac (iinl, 900) linel

write (iout2,900) linel

read (iin1,*) fment 2 , fmext 2 , fmeb2, elbn2

write (iout2, ${ }^{\star}$ ) fment2, fmext2, fmeb2, elbn2

write (iout2, 2021)

$c * \star *$ section $\# 3$ (heat exchanger inlet to outlet) $* * *$

zlhxil - inlet side length leg 1

zlhxi2 - inlet side length leg 2

zlnxo1 - outlet side length leg 1

zlhxo2 - outlet side length leg 2

zltub - tube length

delzhx - change in elevation (out - in)

dhxleg - diameter of leg pipes

diatb. - tube diameter

rght - tube roughness

tuben - number of tubes in a heat exchanger

fmhxti1 - inlet "T" loss leg 1

fmbxto1 - outlet " $T$ " loss leg 1

fmhxti2 - inlet " $T$ " loss leg 2

fmixto2 - outlet "T" loss leg 2

fmindi - inlet header loss

fmhdo - outlet header loss

fmtbi - inlet tube loss

fintio - outlet tube loss

fmeb3 - elbow loss

elbn3_1 - number of elbows on leg 1

elbn3_2 - number of elbows on leg 2

vivlhx - valve loss in leg \#1

vlv2hx - valve loss in leg \#2

tmphxi - heat exchanger inlet side temperature

tmphxo - heat exchanger outlet side temperature

do $i=1,30$

read (iin1,900) linel

enddo

write (iout2, 2030)

read (iinl, 900) linel

write (iout2,900) Iinel

read (iinl,*) zIhxil,zlhxi2,zIhxol,zlhxo2,zltub, delzhx

write (iout2,*) zlhxil,zlhxi2,zlhxo1, zihxo2, zltub, delzhx

read (iinl, 900) linel

write (iout 2,900 ) linel

read (iinl,*) dhxleg, diatb, rght, tuben

write (iout $2, *$ ) dhxleg, diatb, Ight, tuben

read (iinI, 900) linel

write (iout2,900) linel

read (iinl,*) Emhxtil, fmhxto1, fmhxti2, fmhxto2, fmhdi

write (iout2,*) Emhxti1, Emhxto1, fmhxti2, fmhxto2, fmhdi

read (iin1, 900) line1

write (iout2, 900) linel

read (iinl,*) fmindo, fmtbi, fmtbo, fmeb3

write (iout2,*) fmindo, fmtbi, fmtbo, fmeb3 
read (iin1,900) line1

write (iout2,900) line1

read (iin1, *) elbup1, elbup2, elban1, elban2, vlv1hx, vlv2hx

write (iout2, *) elbup1, elbup2, elban1, elbdn2, viv1hx, viv2hx

read (iin1,900) linel

write (iout2,900) line

read (iinl ,*) tmphxi, tmphxo

write (iout2,*) tmphxi, tmphxo

write (iout2, 2031)

c

c

c

$c$

$c$

do $i=1,10$

read (iinl,900) linel

enddo

$c$

write (iout2, 2040)

read (iinl, 900) linel

write (iout2,900) line1

read (iin1,*) $z \operatorname{Ig} 4, \operatorname{diam} 4, \operatorname{deI} z 4$

write (iout2, *) $z \lg 4, \operatorname{diam} 4, \operatorname{del} z 4$

read (iinl, 900) linel

write (iout2,900) 1 inel

read (iin1,*) fment 4 , fmext 4 , fmeb4, elbn4

write (iout2, *) fment 4 , fmext 4 , fmeb4, elbn4

write (iout2, 2041)

c

c

$c$

$c$

$c$

$c$

c

c

$c$

$c$

$c$

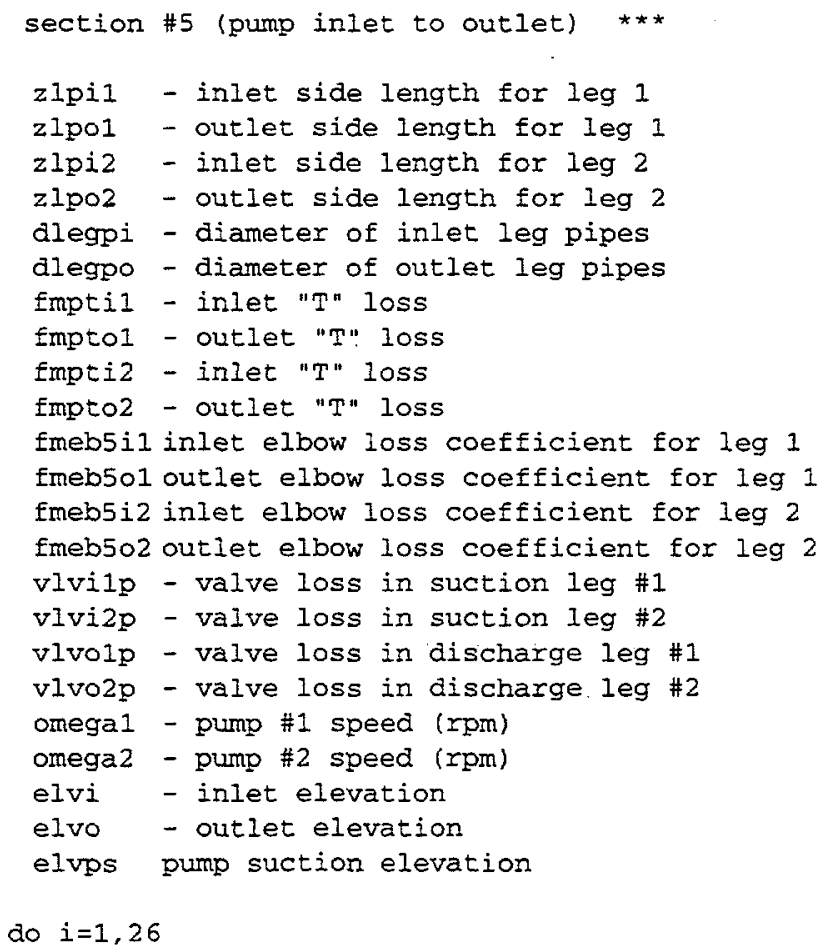

do $i=1,26$ 


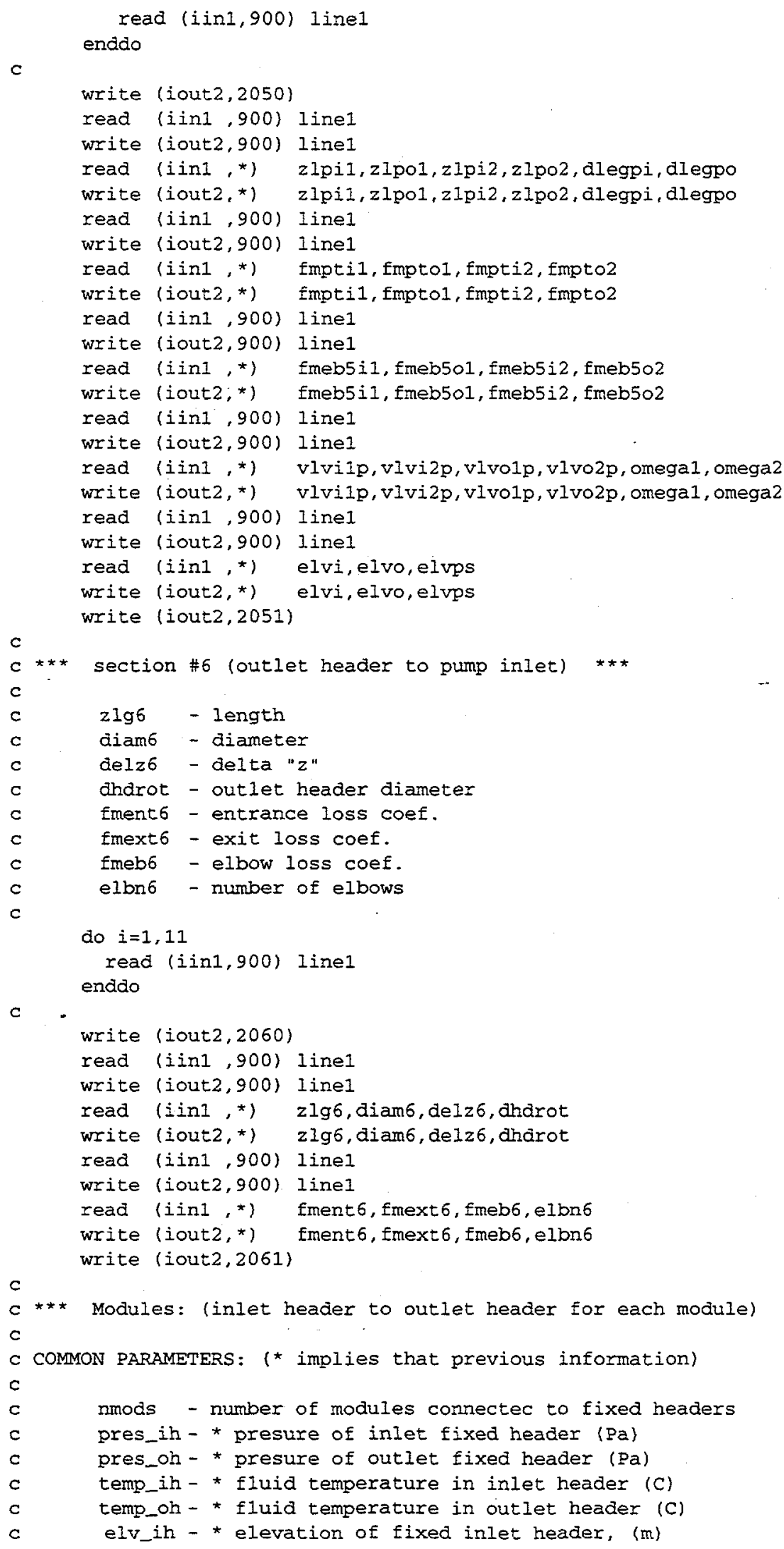


C

c

$c$

$\mathrm{c}$

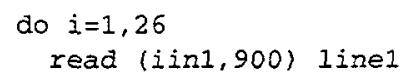




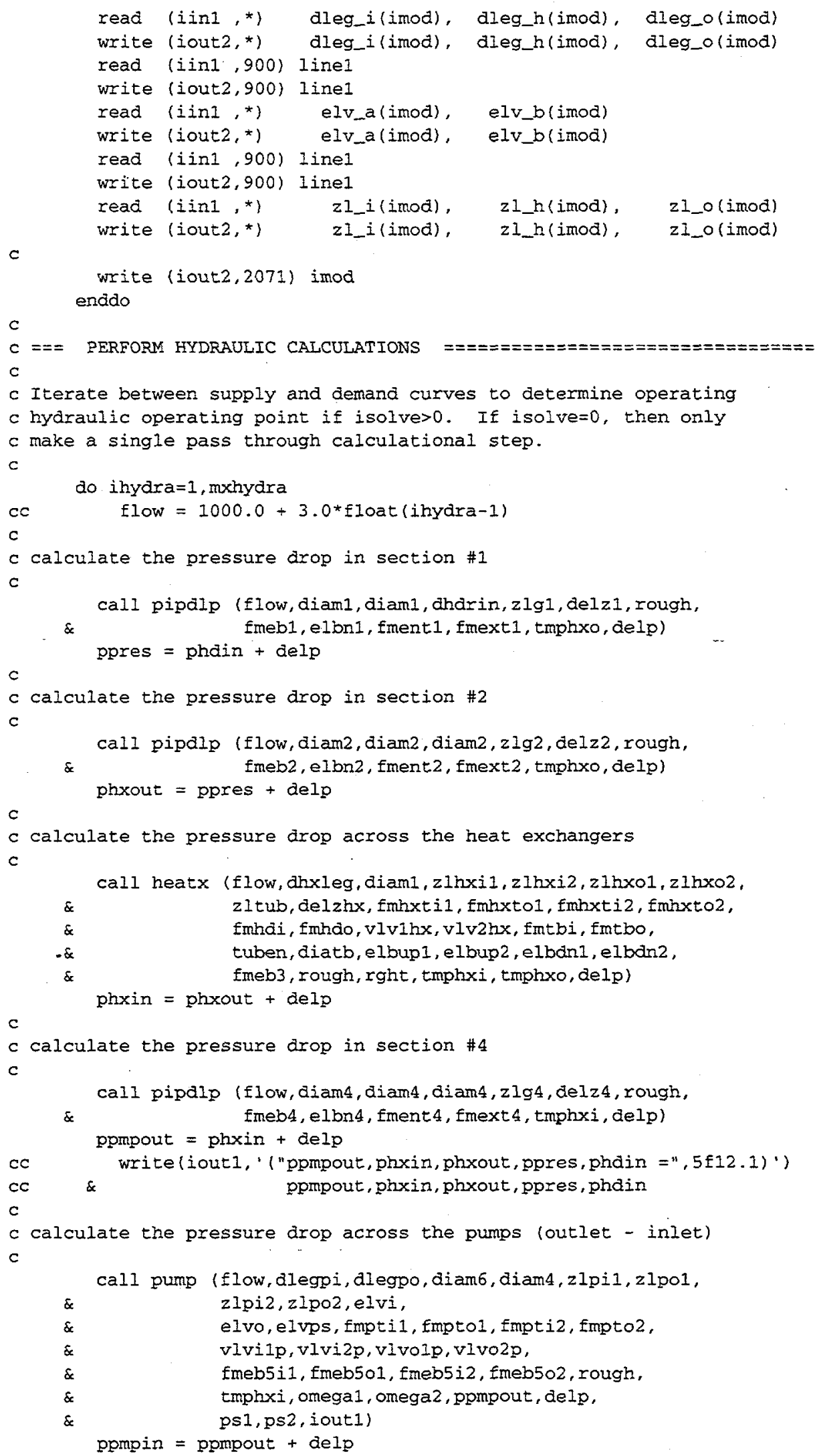


C

c calculate the pressure drop in section \#6

c call pipalp (flow, diam6, dhdrot, diam6, zlg6, delz6, rough,

$\alpha$ fmeb6, elbn6, fment6, fmext6, tmphxi, delp)

c phdout $=$ ppmpin + delp

c Compute mass flowrates through each module connected to the common

$c$ inlet \& outlet headers.

$c$

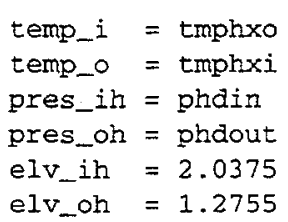

c

if (isolve.gt.0) then

call module (nmods, pres_ih,pres_oh, temp_i, temp_o,

$\&$
$\&$
$\&$
$\&$
$\&$
$\&$

kform_i, kform_h, kform_o, kvalv_i,

kvalv_h, kvalv_o, kebow_i, kebow_h, kebow_o, nebow_i, nebow_h, nebow_o, kent, kext,

dleg_i, dleg_h, dleg_o,elv_ih, elv_oh,

elv_a,elv_b,zl_i,zl_h,zl_o,

endif

rough, flowm, pres_a,pres_b, ftot, iout 1 )

$c$

c Check for hydraulic supply \& demand curve convergence.

c

dflow $=$ flow - ftot

write (25, ' ("ihydra, delp, flow, ftot $=$ ", i5, 5x, 3f10.3) ')

$\&$

ihyara, (phdin-phdout) *pconv, flow, ftot

write $(26$, ' ("ihydra, flow, delps $=$ ", i5, $2 x, 8 f 8.2$ )')

\& inydra, flow, (phdout-ppmpin)*pconv,

(ppmpin-ppmpout) *pconv, (ppmpout-phxin) *pconv,

$\begin{array}{ll}\text { \& } & \text { (phxin-phxout) }{ }^{*} \text { pconv, (phxout-ppres) }{ }^{\star} \text { pconv, } \\ \& & \text { (ppres-phdin) *pconv, (phdin-phdout) }{ }^{\star p} \text { ponv }\end{array}$

if (abs (aflow).1t.hconvg .or. isolve.eq.0) then

goto 10

else

flow $=$ weight $*$ low + (one-weight $) *$ ftot

endif

enddo

$c$

c Solution failed to converge within specified number of iterations,

c notify then terminate execution.

c

write (iout1, 800) mxhydra, flow, ftot, dflow

stop

$c$

c solution converged or no iteration requested, write out final

c solution.

c

10 continue

c

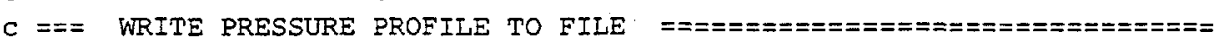

c

write (iout1,950) ihydra, Elow, ftot

write (iout1,1001) phdout*pconv

write (iout1, 1002) ppmpin*pconv

write (iout1, 1008) ps I*pconv

write (iout1, 1009) ps2*pconv 
write (iout 1, 1003) ppmpout*pconv

write (iout 1, 1004) phxin*pconv

write (iout1, 1005) phxout*pconv

write (iout1,1006) ppres*pconv

write (iout1,1007) phain*pconv

write (iout1, 1018)

totpd $=($ phdin - phdout $) *$ pconv

write (iout1, 1019) totpd

c

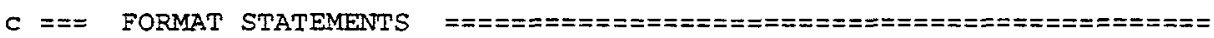

$\mathrm{c}$

800 format $(/ /, 1 * * * * * * * * *$ failed to converge $* * * * * * * * * * * * * \cdot, 1 /$,

900 format $(280)$ ihydra, flow, ftot, dflow $=$, i5, 3f10.3)

910 format $(/, a 80, /)$

950 format (' \#hydro iterations=',i3,' süpply flow=', f7.1,

\& demand $\mathrm{flow}={ }^{\prime}, \mathrm{f} 7.1,1$

c

1001 format (1x, 'outlet header pressure $=', g 16.8$, ' psia' ')

1002 . format ( $1 \mathrm{x}$, 'pump inlet pressure $\quad=', 916.8$,' psia' ')

1008 format ( $1 \mathrm{x}$, 'pump \#1 suction pressure $=\cdot, 916.8$,' psia ')

1009 format (1x, 'pump \#2 suction pressure $=\cdot, 916.8$, ' psia ')

1003 format ( $1 \mathrm{x}$, 'pump outlet pressure $=$ ", g16.8,' psia ')

1004 format $(1 \mathrm{x}$, ' $\mathrm{HX}$ inlet pressure $\quad=\prime, 916.8$, ' psia')

1005 format (1X,'HX outlet pressure $\quad=', g 16.8, '$ psia ')

1006 format ( $1 \mathrm{x}$, ' Pressurizer pressure $\quad=\prime, 916.8,{ }^{\prime}$ psia ')

1007 format ( $1 x$, 'Inlet Header pressure $\quad=\prime, 916.8$, 'psia ')

1018 format (' ')

1019 format (1x, 'Header to Header pres drop =',g14.7,' psid")

c

2010 format $(/, \cdot * \star * \star \star * * *$ PRESSURIZER -TO- INLET HEADER $\star * \star * * * * * 1)$

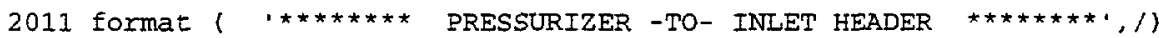

2020 format $(/, \cdot * * *$ HEAT EXCHANGER OUTLET -TO- PRESSURIZER $* * * * \cdot)$

2021 format $(\cdot * * *$ HEAT EXCHANGER OUTLET -TO- PRESSURIZER $* \star * * 1,1)$

2030 format $(/, \cdots * * * *$ HEAT EXCHANGER INLET -TO- OUTLET $* * \star \star * \star * \cdot$ )

2031 format ( $* * * * * *$ HEAT EXCHANGER INLET -TO- OUTLET $* * * * * * * \cdot, 1)$

2040 format $(/, * * * *$ PUMP OUTLET - TO- HEAT EXCHANGER INLET $* * * * \cdot)$

2041 format (,$* * * *$ PUMP OUTLET -TO- HEAT EXCHANGER INLET $* * * * 1,1)$

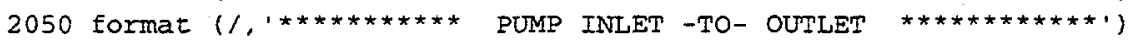

2051 format (,$* * * * * * * * * * *$ PUMP INLET -TO- OUTLET $* * * * * * \star * * * * * \cdot, 1)$

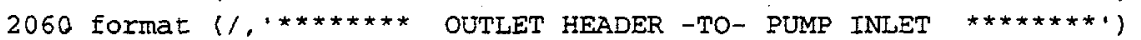

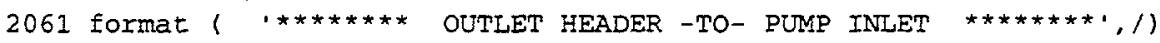

2069 format $(/$,

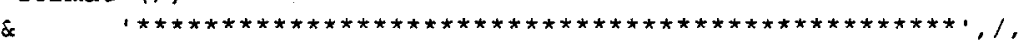

$\& \quad * \quad, i 2,1$ MODULES CONNECTED TO INLET/OUTLET HEADER $*, 1,1$,

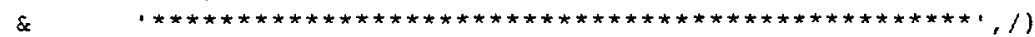

2070 format $(/$,

\& $\cdot \star \star \star \star \star$ MODULE ', i2, $:$ INLET -TO- OUTLET HEADER $\star \star \star \star \star *)$ )

2071 format

$\& \quad \cdot * \star * * *$ MODULE $\cdot, i 2, '$ : INLET -TO- OUTLET HEADER $\star * \star \star * *, / 1)$

c

stop

end

\section{Subroutine frict:}

subroutine frict (re,ed,zf)

c

c

c This subroutine calculates the Moody friction factor for a

c tube. Inputs are the Reynolds \#, and the relative roughness.

c The Darcy friction factor is returned. 


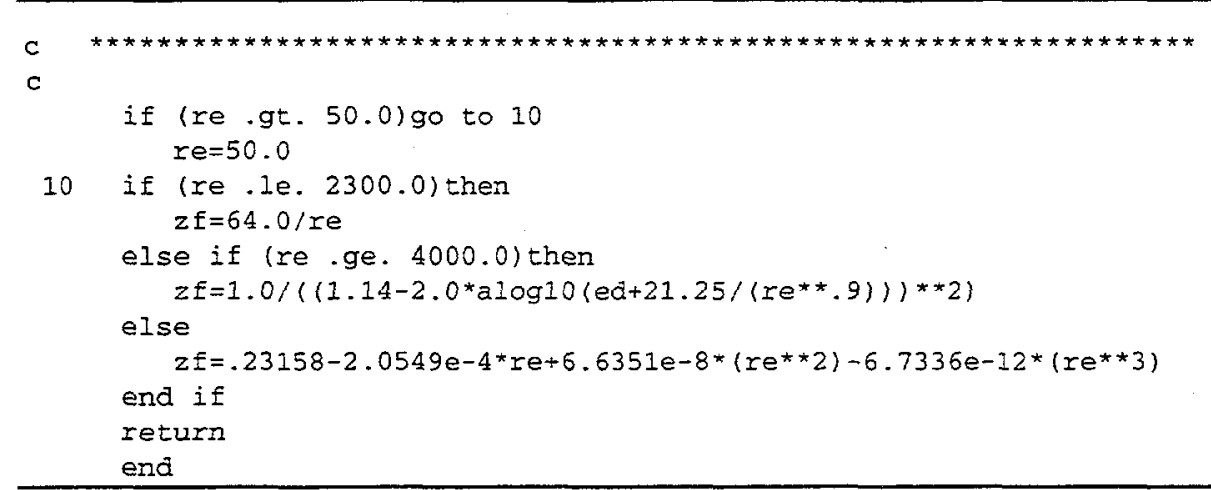

\section{Subroutine heatx:}

subroutine heatx(flow, dleg, dmpip, zli1,zli2,zlo1,zlo2,

\section{$\&$}

$\&$

$\bar{\varepsilon}$

$\&$

zltub, delz, fmltil, fmltol, fmlti2, fmlto2, fmhi, fmho, zkvlv1, zkv1v2, fmti, fmto,

tuben, diat, elbup1, elbup2, elbdn1, elbdn2, fmeb90, rough, rght, tin, tout, delp)

$\mathrm{c}$

$c$

c

c INPUT

mass flowrate

pipe diameter in the two legs

diameter of main pipe

length of pipe upstream of hx 1

length of pipe upstream of $\mathrm{hx} 2$

length of pipe downstream of $\mathrm{hx} 1$

length of pipe downstream of $h \times 2$

tube length

delz elevation change (outlet-inlet)

fmltil entrance "T" form loss for leg 1

fmltol exit "T" form loss leg 1

fmlti2 entrance "T" form loss leg 2

fmltó2 exit "T" form loss leg 2

fmil heat exchanger entrance form loss

fmh heat exchanger exit form loss

zkvlv1 valve loss coefficient for leg \#1

zkvlv2 valve loss coefficient for leg \#2

finti $h x$ tube entrance form loss

fmto $h x$ tube exit form loss

tuben number of tubes in $h x$

diat tube diameter

elbupl number of elbows on leg 1 upstream of $\mathrm{HX}$

elbup2 number of elbows on leg 2 upstream of $\mathrm{HX}$

elbanl number of elbows on leg 1 down stream of $\mathrm{HX}$

elban2 number of elbows on leg 2 down stream of $\mathrm{HX}$

fmeb90 elbow form loss (90 degrees)

rough pipe roughness

rght tube roughness

tin inlet temperature

tout outlet temperature

iout2 output file number

implicit real $(a-h, 0-z)$, integer $(i-n)$ 
C

Page:

c

parameter (zero=0.0, half $=0.5, t w o=2.0$ )

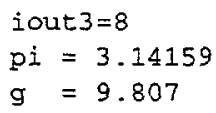


call frict(re, relrgh, f)

$\mathrm{ft} 2=\mathrm{f}$

Calculate the " $T$ " form losses in terms of the leg velocities and as functions of the flow splits:

c

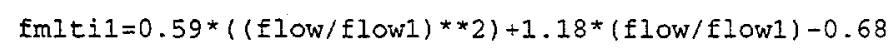

$\mathrm{c}$

write (iout3, 800) fmitil, fmlti2, fmltol, fmito2

800 format $(1 \mathrm{x}, 4 \mathrm{~g} 16.8)$

c

c Leg 1

$\mathrm{c}$

upstrm $=(f m l t i l+E m h i+e l b u p 1 * f m e b 90+(f i 1 * z l i 1) /$ aleg $) /$

$\&$ (deni*dleg*dleg*dleg*dleg)

anstrm $=($ fmho $+e l b a n l * f m e b 90+z k v l v 1+f$ mexp $+(f \circ 1 * z l o l) / \mathrm{dleg}) /$

$\& \quad$ (deno*dleg*dleg*dleg*dleg) +

\& (fmlto1/(deno*dmpip*dmpip*dmpip*ampip))

tubios $=\left(\right.$ two $^{*}($ fmti+fmto $)+(f t 1 * z l t u b) /$ diat $) /$

$\&$ (denb*tuben*tuben*diat*diat*diat*diat)

$c$ $c 1=u p s t r m+$ anstrm+tublos

c Leg 2

$c$

upstrm $=(f m 1 t i 2+f m i+e l b u p 2 * f m e b 90+(f i 2 * z l i 2) / d l e g) /$

\& (deni*dleg*dleg*dleg*dleg)

anstrm $=($ fmho+elbdn2 2 fmeb $90+z k v 1 v 2+f m e x p+(f \circ 2 * z l o 2) / d l e g) /$

\& $\quad$ (deno*dleg*aleg*aleg*aleg) +

\& (fmIto2/(deno*dmpip*ampip*dmpip*dmpip))

tublos $=($ two* $($ fmti+fmto $)+($ ft $2 * z i t u b) /$ diat $) /$

\& - (denb*tuben*tuben*aiat*diat*diat*diat)

c2 =upstrm+dnstrm+tublos

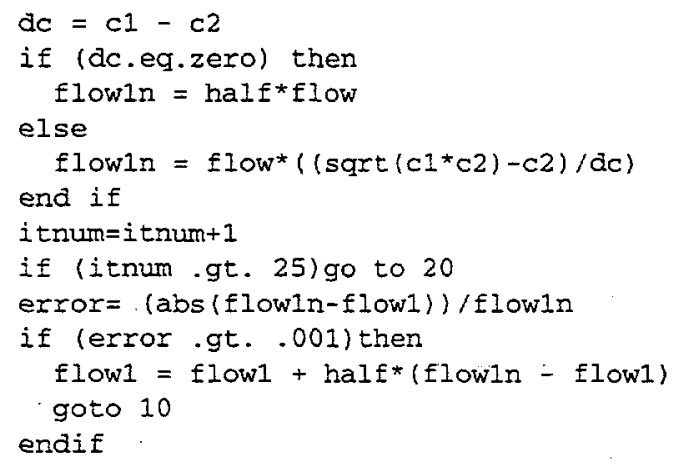


\& $\quad\left(\left(1.0 /\left(\operatorname{deno}^{*}\left(\operatorname{dmpip}{ }^{*} 4\right)\right)\right)-\left(1.0 /\left(\operatorname{deni}^{*}\left(\operatorname{dleg}^{* \star 4}\right)\right)\right)\right)$

write $(27$, ' ("itnum, flow1, delp, c1,c2 = ", i3, 4f10.3)') itnum, flow1, delp, c1, c2

c

C

calculate and write out the heat exchanger pressure drops:

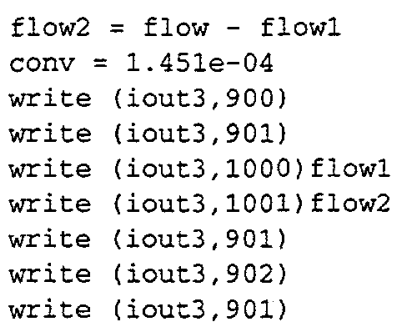


$\mathrm{c}$

write (iout3, 901)

write (iout 3,905 )

write (iout 3,901 )

deltaz $=1.5$

$d p 1=($ vsqtt $1 *($ fmti+fmto $)-d e n b * g *$ deltaz $) *$ conv

dp2 $=\left(\right.$ vsqtt $2 *($ fmti $i$ fmto $)-d e n b^{*} g^{*}$ deltaz $) *$ conv

dp I sum $=$ dp 1 sum $+d p 1$

dp2sum=dp2sum+dp2

write (iout3,1002) dp 1

write (iout 3,1003$) \mathrm{dp} 2$

write (iout3, 901)

C

pressure drop in second pass tubes:

write (iout 3,901 )

write (iout3, 905)

write (iout 3,901 )

$\operatorname{dp} I=($ vsqtt $1 *(($ ft $1 * 21$ tub*0.5)/diat $)) * \operatorname{conv}$

$\operatorname{dp} 2=($ vsqtt $2 *((f t 2 * z l t u b * 0.5) /$ diat $)) * \operatorname{conv}$

$d p I$ sum $=d p 1$ sum $+d p 1$

$\mathrm{dp} 2 \mathrm{sum}=\mathrm{dp} 2 \mathrm{sum}+\mathrm{dp} 2$

write (iout3,1002) dp 1

write (iout3, 1003) dp2

write (iout3,901)

c

C

c

pressure drop from tube exit to down stream of the exit header:

write

write (iout3, 901 )

vsqt $1=(8.0 *$ flow $1 *$ flow 1$) /($ deno*pi*pi* $($ dleg**4))

vsqt $2=(8.0 *$ flow $2 *$ flow 2$) /($ deno*pi*pi* $($ dleg**4)$)$

$d p 1=($ vsqt $1 *($ fmho +1$)+v s q t t 1 *($ Imto -1.0$)) * \operatorname{conv}$

$d p 2=(v s q t 2 *($ fmho +1$)+v s q t t 2 *($ fmto -1.0$)) * \operatorname{conv}$

$d p I$ sum=dplsum $+d p 1$

dp2 sum $=$ dp 2 sum $+d p 2$

write (iout3,1002) dp1

write (iout3,1003) dp2

write (iout3,901)

pressure drop from down stream of outlet header to

down stream of the "T":

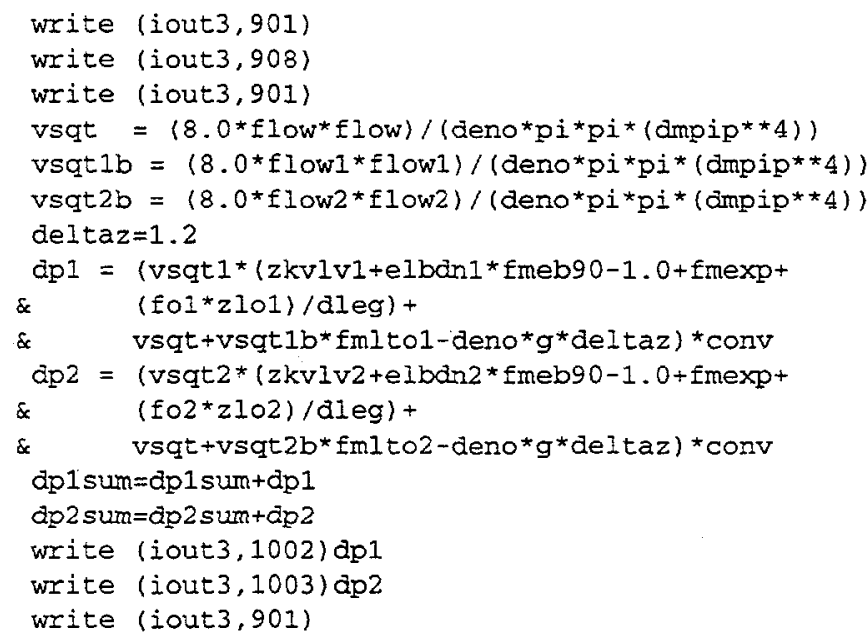


write (iout3, 1004) dplsum

write (iout3, 1005) dp2sum

write (iout 3,901 )

dprtn $=$ delp*conv

write (iout3, 1006) dprtn

c

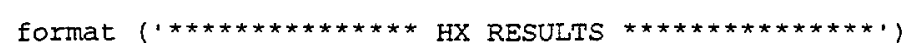

\section{Subroutine module:}

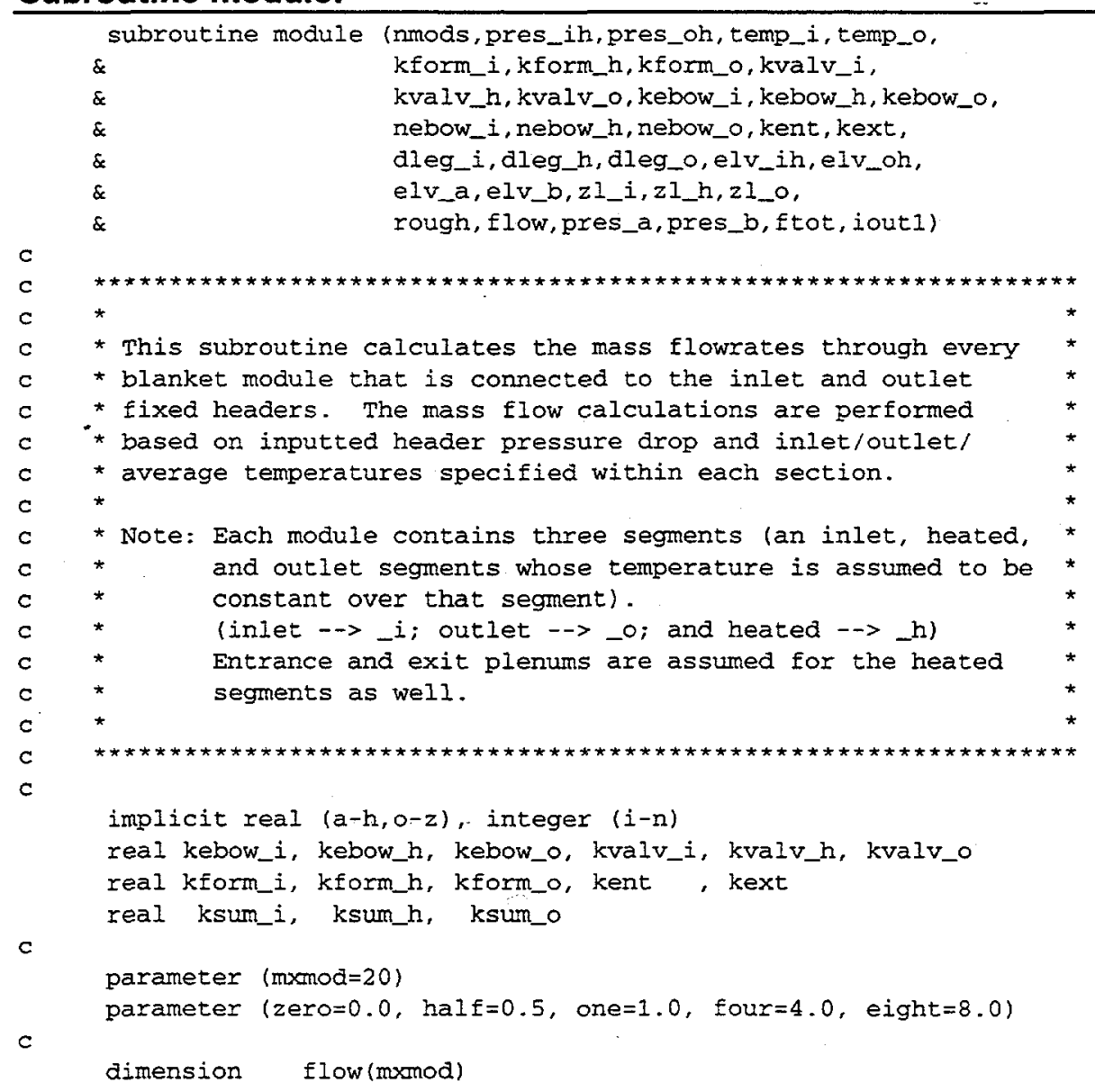


dimension pres_a(mxmod), pres_b(mxmod)

dimension kent (mxmod), kext (mxmod)

dimension kform_i (mxmod), kform_h(mxmod), kform_o(mxmod)

dimension kvalv_i (mxmod), kvalv_h (mxmod), kvalv_o(mxmod)

dimension kebow_i (mxmod), kebow_h (mxmod), kebow_o(mxmod)

dimension nebow_i (mxmod), nebow_h (mxmod), nebow_o(mxmod)

dimension dleg_i (mxmod), dieg_h (mxmod), dleg_o(mxmod)

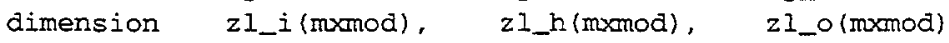

dimension elv_a(mxmod), elv_b(mxmod)

INPUT :

nmods - number of modules connectec to fixed headers

pres_ih-presure of inlet fixed header (Pa)

pres_oh - presure of outlet fixed header (Pa)

temp_i - average fluid temperature in inlet segment. (C)

temp_h - average fluid temperature in inlet segment (C)

temp_o - average fluid temperature in inlet segment (C)

kform_i - inlet segment form losses (unitless)

kform_h - outlet segment form losses (unitless)

kform_o - heated segment form losses (unitless)

kvalv_i - valve loss coefficient for inlet segment (unitless)

kvalv_h - valve loss coefficient for inlet segment (unitless)

kvalv_o - valve loss coefficient for inlet segment (unitless)

kebow_i - elbow form loss inlet leg (less than 180 degrees)

kebow_h - elbow form loss heated leg (less than 180 degrees)

kebow_o - elbow form loss outlet leg (less than 180 degrees)

nebow_i - number of elbows in inlet leg (unitiess)

nebow_h - number of elbows in heated leg (unitless)

nebow_o - number of elbows in outlet leg (unitiess)

kent - Entrance loss coefficient at inlet header (unitless)

kext - Exit loss coefficient at outlet header (unitless)

dleg_i - pipe diameter on inlet leg (m)

dleg_h - pipe diameter on heated leg (m)

dleg_o - pipe diameter in outlet leg (m)

elv_ih - elevation of fixed inlet header, (m)

elv_a - elevation of entrance to heated segment ( $m$ )

elv_b - elevation of exit to heated segment (m)

elv_on - elevation of fixed outlet header, (m)

$z z_{-} i$ - Lenght of piping in inlet segment (m)

$z 1$ m - Lenght of piping in heated segment (m)

$z 1$ - - Lenght of piping in outlet segment (m)

rough - pipe roughness $(\mathrm{m})$

OUTPUT :

flow - array of mass flowrates per module $(\mathrm{kg} / \mathrm{s})$

ftot - total mass flowrate from all modules ( $\mathrm{kg} / \mathrm{s}$ )

pres_a - pressure at entrance of heated segment (Pa)

pres_b - pressure at exit of heated segment (Pa)

P_conv $=14.696 / 101325.0$

pi $=\operatorname{acos}(-$ one $)$

$\mathrm{g}=9.807$

fac $=$ eight $/\left(g^{\star} p i{ }^{*} \mathrm{p} i\right)$

conv $=1.0 e-4$

eps $\quad=1.0 \mathrm{e}-3$

maxit $=1000$

fric $=0.015$

write (iout 1, 130) 
$\mathrm{C}$

c Check for exceeding allowable number of modules.

if (nmods.gt.mxmod) then

write (iout1, ' $/ / /$,

\& " ERROR: maximum number of modules allowed $=", i 4)$ ") mxmod stap

endif

$c$

c Calculate fluid properties for each segment. Note that each model

$c$ considered to be operating at similar temperatures as neighbors.

temp_h $=$ half* $\left(t e m p \_i+\right.$ temp_o $)$

call propw (temp_i,den_i,vis_i)

call propw (temp_h, den_h,vis_h)

call propw (temp_o, den_o,vis_o)

c Compute total dymanic head drop between inlet and outlet headers.

$c$ we are assuming that inlet and outlet header velocities are

c appproximately equal.

$c$

dthd $=\left(p r e s \_i h / d e n \_i-p r e s \_o h / d e n \_o\right) / g+\left(e l v \_i h-e l v \_o h\right)$

$c$

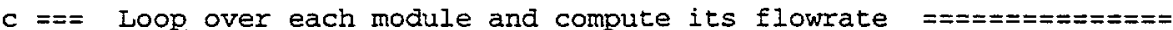

$\mathrm{c}$

do imod=1, nmods

c sum up all form-type losses per segment.

$k s u m_{-} i=k$ form_i(imod) $+k v a l v_{-} i(i m o d)+$

float (nebow_i (imod))*kebow_i (imod) + kent (imod)

$k s u m \_h=k f o r m \_h(i m o d)+k v a l v \_h(i m o d)+$

$\&$

float (nebow_h(imod))*kebow_h(imod)

ksum_o $=$ kform_o(imod) $+k v a l v \_o(i m o d)+$

\& float (nebow_o(imod))*kebow_o(imod) + kext (imod)

$\mathrm{C}$

$c$ Estimate initial guess for flow split based on form losses with a

c constant friction loss term (i.e., fully turbulent assumption).

$\mathrm{C}$

- rdenom_i $=\mathrm{fac} /\left(\mathrm{den} \_i * * 2 * d \mathrm{leg}_{-} i(\operatorname{imod}) * * 4\right)$

rdenom_h $=\mathrm{fac} /($ den_h $\mathrm{h} * 2 *$ dieg_h $($ imod $) * * 4)$

rdenom_o $=\mathrm{fac} /\left(\right.$ den_o**$_{2} *$ dleg_o $($ imod $\left.) * * 4\right)$

ratio_i $=21+i(i m o d) / d e_{e}+i(i m o d)$

ratio_h = zI_h(imod)/dleg_h(imod)

ratio_o = zl_o(imod)/dleg_o(imod)

sum_i $=$ rdenom_i* (ksum_i + fric*ratio_i)

sum_h = rdenom_h* (ksum_h + fric*ratio_h)

sum_o $=$ rdenom_o* $\left(\mathrm{ksum} \_0+\right.$ fric*ratio_o $)$

sum $\quad$ sum_i + sum_h + sum_o

flo2 $=\mathrm{dthd} / \mathrm{sum}$

$c$

if (f102.ge.zero) then

flow (imod) $=\operatorname{squt}(\mathrm{flo})$

else

Elow (imod) $=-\operatorname{sqrt}(-f l 02)$

endif

$c$

$c===$ For current module solve non-linear flow equation $============$

c

do iter $=1$, maxit

c 
c Calculate function at current iterate \& perturbation.

$c$

c Calculate perturbed flows

c

$$
\begin{aligned}
& \text { flo }=\text { flow }(\text { imod }) \\
& \text { flop }=\text { flo* (oneteps) }
\end{aligned}
$$

$c$

c Update friction factors

$c$

$c$ Inlet segment:

relrgh $=$ rough/dleg_i (imod)

re $\quad=($ four*flo $) /\left(p i * v i s_{-i}{ }^{*}\right.$ dleg_i (imod) $)$

rep $=r e^{*}$ (one+eps)

call frict (re, relrgh, fli)

cc write (iout1, '(" $\mathrm{f}_{-} i=$ ",f8.4)') $\mathrm{f}_{-}$

call frict (rep,relrgh, f_ip)

c Heated segment:

relrgh $=$ rough/dleg_h $($ imod $)$

re $=\left(\right.$ four*flo) $/\left(p i * v i s \_h * a l e g \_h(i m o d)\right)$

rep $=r e^{*}$ (one+eps)

call frict (re, relrgh, f_h)

cc

write (iout1, "(" $\mathrm{f}_{\text {h }} \mathrm{h}=$ ", f8.4)') E_h

c Outlet segment:

call frict ( $x e p$, relrgh, f_hp)

relrgh $=$ rough $/$ dleg_o $($ imod $)$

re $\quad=($ four*flo $) /\left(p i * v i s \_o *\right.$ dleg_o (imod) $)$

rep $=r e^{*}$ (one+eps)

call frict (re, relrgh, f_o)

cc write (iout1, (" $\left.f_{-} 0=", f 8.4\right)^{\prime}$ ) f_o

cal1 Erict (rep, relrgh, f_op)

c

$c$ Calculate function at current iterate point

c

sum_i = rdenom_i* (ksum_i + f_i*ratio_i)

sum_h $=$ rdenom_h* $(\mathrm{ksum} h+\mathrm{f}$. $h *$ ratio_h $)$

sum_o $=$ rdenom_o* (ksum_o + f_o*ratio_o $)$

sum $=$ sum_i + sum_h + sum_o

Eunc $=$ Elo*flo*sum - dthd

c.

$c$ Calculate function at its perturbation point

c

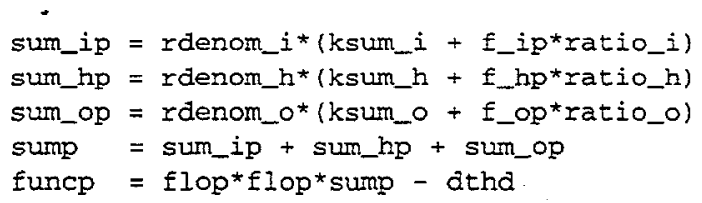


ThAC B.

$c$ notify then terminate execution.

$c$ write (iout1,205) imod, iter, maxit, flow, dflow stop

$c$

c Solution converged, compute entrance and exit plenum pressures to

$c$ the heated segment and then loop to next module.

c

10

continue

c Entrance plenum pressure:

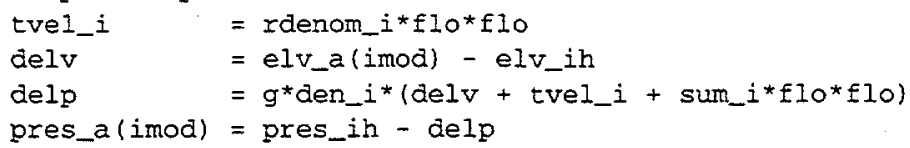

\section{Subroutine npsha:}


subroutine npsha (tfld,pfld, rhof, velf,a_npsh)

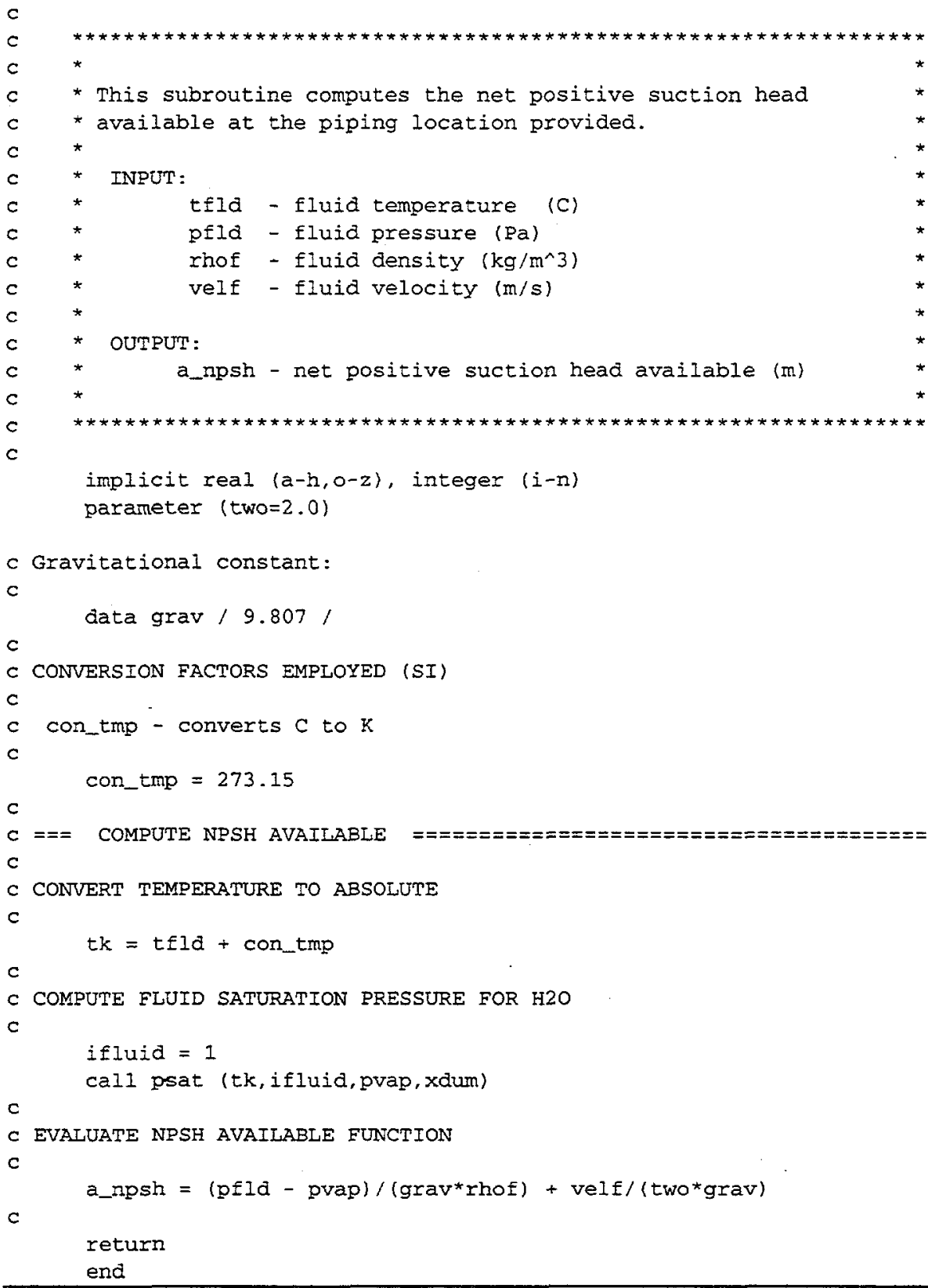

\section{Subroutine npshr:}

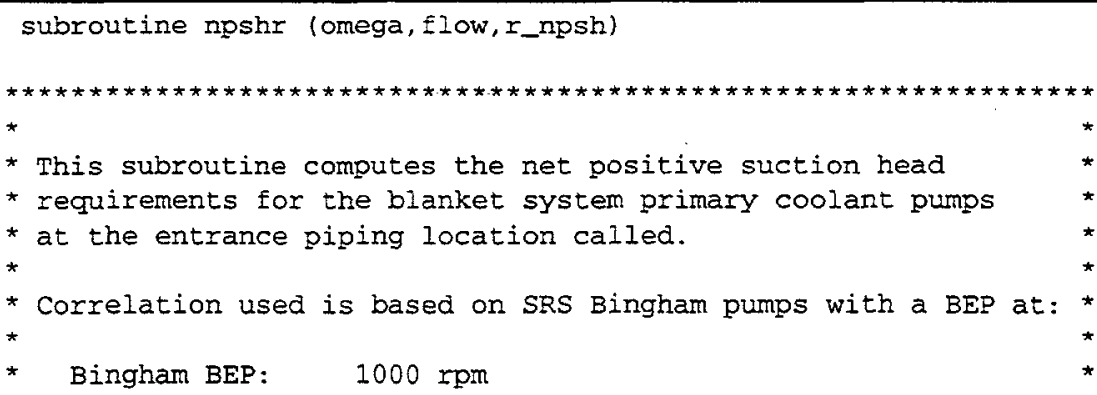




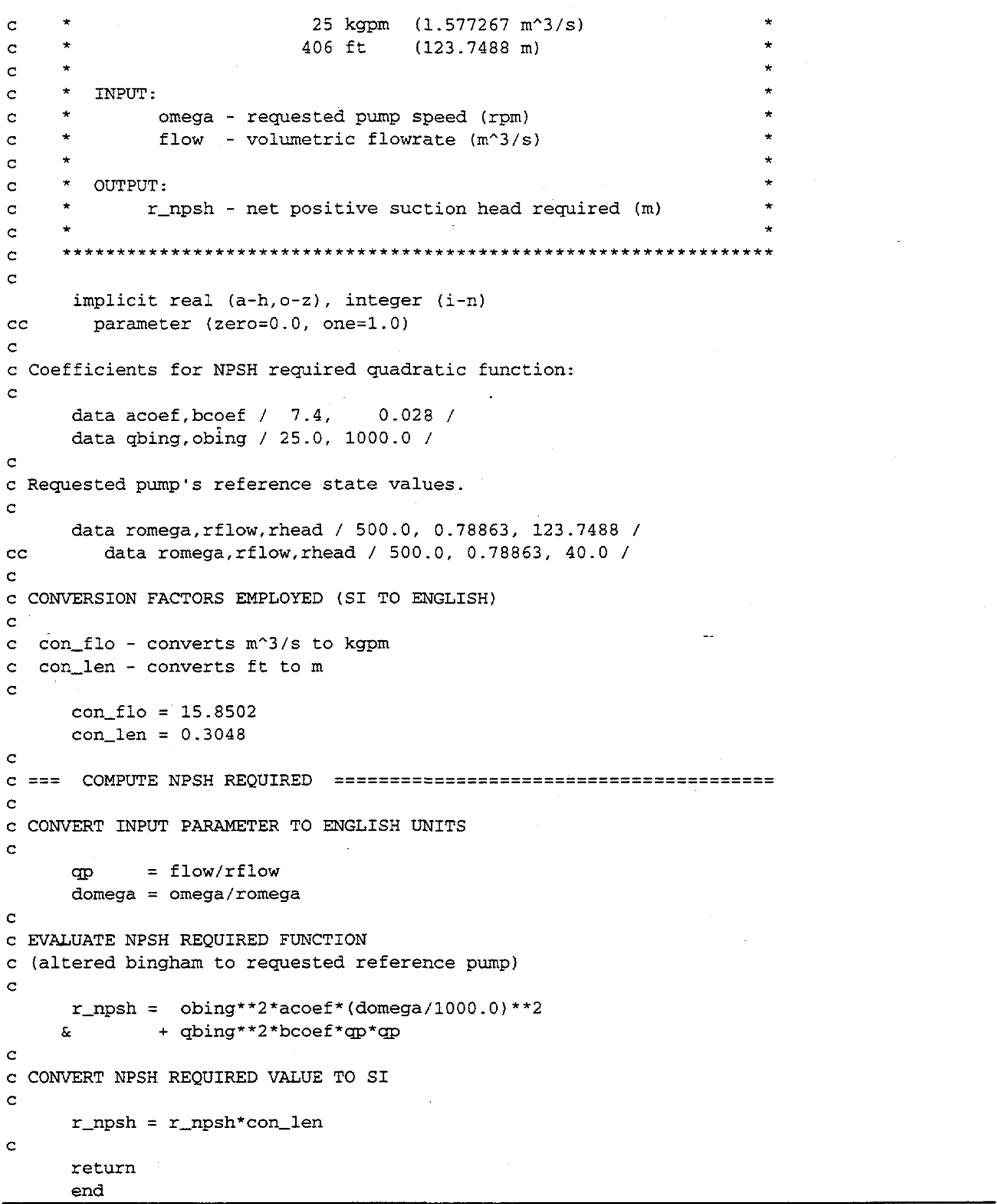

\section{Subroutine pipdlp:}

subroutine pipdlp(flow, diam, diamin, diamot, zlngth, delz, rough,

fmeb90, elbn90, fment, fmext, temp, delp)

This subroutine calculates the pressure drop in a length of pipe.

The units are SI. 
INPUT :

flow mass flowrate

diam pipe diameter

diamin inlet diameter

diamot outlet diameter

zlngth pipe length

delz change in elevation $\{z$ (downstream) $-z$ (upstream)

rough pipe roughness

fmeb90 elbow form loss (90 degrees)

elbn90 number of $90 \mathrm{deg}$. elbows

fment entrance form loss

fmext exit form loss

$g$ acceleration of gravity

temp fluid temperature

$$
\begin{aligned}
p i & =3.14159 \\
g & =9.807
\end{aligned}
$$

$c$

Calculate the friction factor

call propw(temp, den, vis)

re $\quad=(4.0 *$ flow $) /(\mathrm{pi} * v i s *$ diam $)$

relrgh $=$ rough/diam

call frict(re,relrgh, f)

elbn $45=0.0$

fmeb $45=0.0$

Calculate the pressure drop

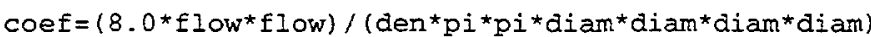

bern $=((8.0 *$ flow*flow $) /($ den*pi*pi $)) *$

$\& \quad(1.0 /(\operatorname{diamot} * \star 4))-(1.0 /(\operatorname{diamin} * * 4)))$

frmsum $=e l b n 90$ *meb $90+e l b n 45$ *meb45+fment+fmext

delp $=($ den* $g * \operatorname{del} z)+$ bern+coef* $($ frmsum $+(f * z l n g t h) /$ diam $)$

return

end

\section{Subroutine pmphc:}

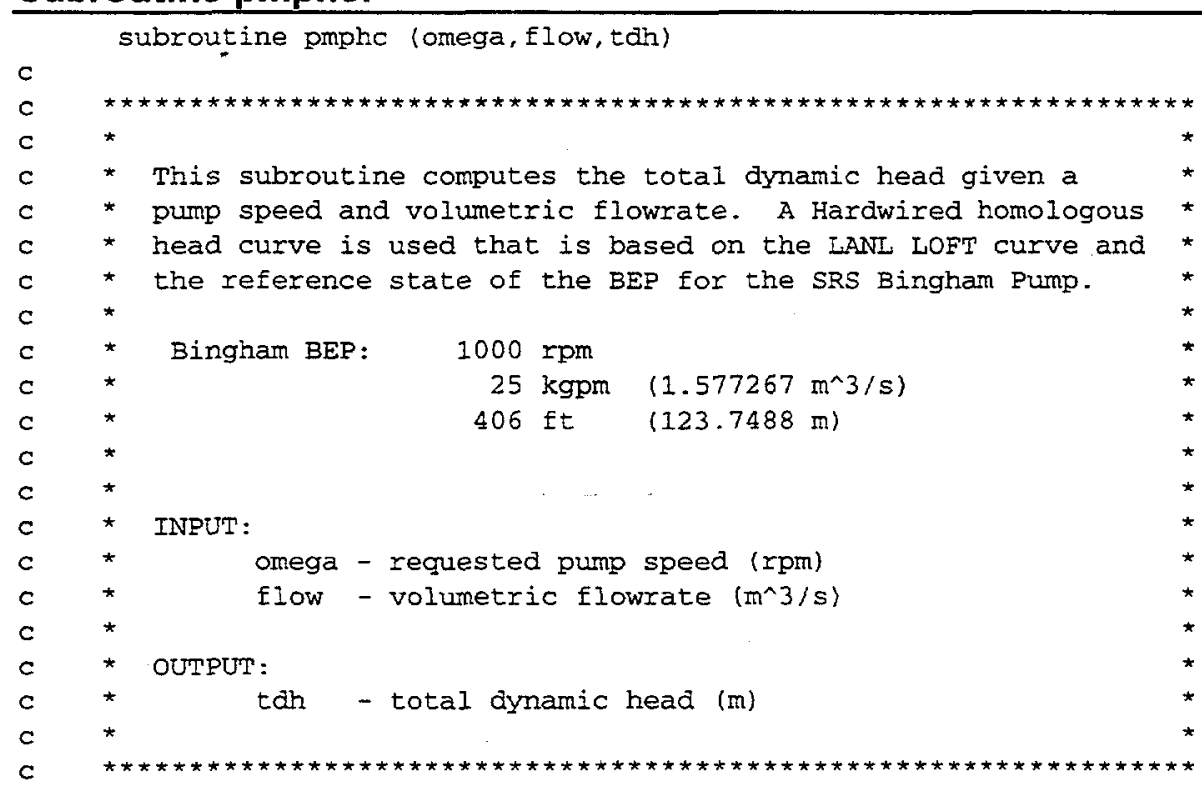


implicit real $(a-h, o-z)$, integer $(i-n)$

parameter (one=1.0, two=2.0)

c Bingham BEP Reference conditions (SI units):

data romega, rflow, rhead / 500.0, 0.78863,40.0/

data romega, rflow, rhead / 1000.0, 1.577267, 123.7488/

data romega, Iflow, thead / 314.16, 0.51546, 54.68951 /

Quadratic polymomial fit of LANL LOFT Homologous head curve coefs:

data acoef, bcoef, ccoef $/ 1.4,-0.15,-0.25 /$

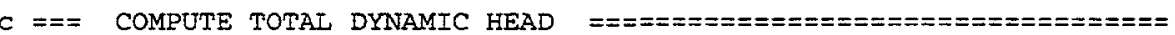

C NORMALIZE INPUT PARAMETERS

$c$

d_omega $=$ omega $/$ romega

d_flow $=$ flow/xflow

$x$ homo $=$ d_flow/d_omega

EVALUATE HOMOLOGOUS FUNCTION

if (x_homo.Ie.one) then

either a quadratic fit or Iinear interpolation:

$y$ _homo $=$ acoef $+x$ _homo* $($ bcoef $+\operatorname{ccoef} * x$ homo $)$

if (x_homo.1e. 0.4 ) then

$y_{\text {_homo }}=1.3+0.25 *\left(0.4-x_{\text {hlomo }}\right)$

else

$y_{\text {_homo }}=1.0+0.5^{*}\left(1.0-x_{\text {hhomo }}\right)$

else endif

$y_{\text {_homo }}=$ one $+\left(\right.$ bcoef + two ${ }^{*}$ coef $) *(x$ homo - one $)$

endif

$c$

C SCALEUP TO THD VALUE

$c$

- $t d h=$ y_homo*d_omega ${ }^{\star}$ d_omega ${ }^{\star}$ rhead

$t \mathrm{dh}=107.0$

$t a h=0.0$

return

end

\section{Subroutine propw:}

subroutine propw (temp, den, vis)

c

This subroutine returns properties for liquid

water as a function of temperature, (deg. C)

parameter $\{a 1=1004.39, a 2=-0.13226, a 3=-3.053 e-3$ )

parameter $(b 1=1.4051 e-3, b 2=-2.2076 e-5, b 3=1.0849 e-7)$

$c$

den $=a 1+a 2$ *emp $+a 3 *$ temp *temp

vis $=b 1+b 2$ *temp $+b 3$ * temp * temp

return

end 
Subroutine psat:

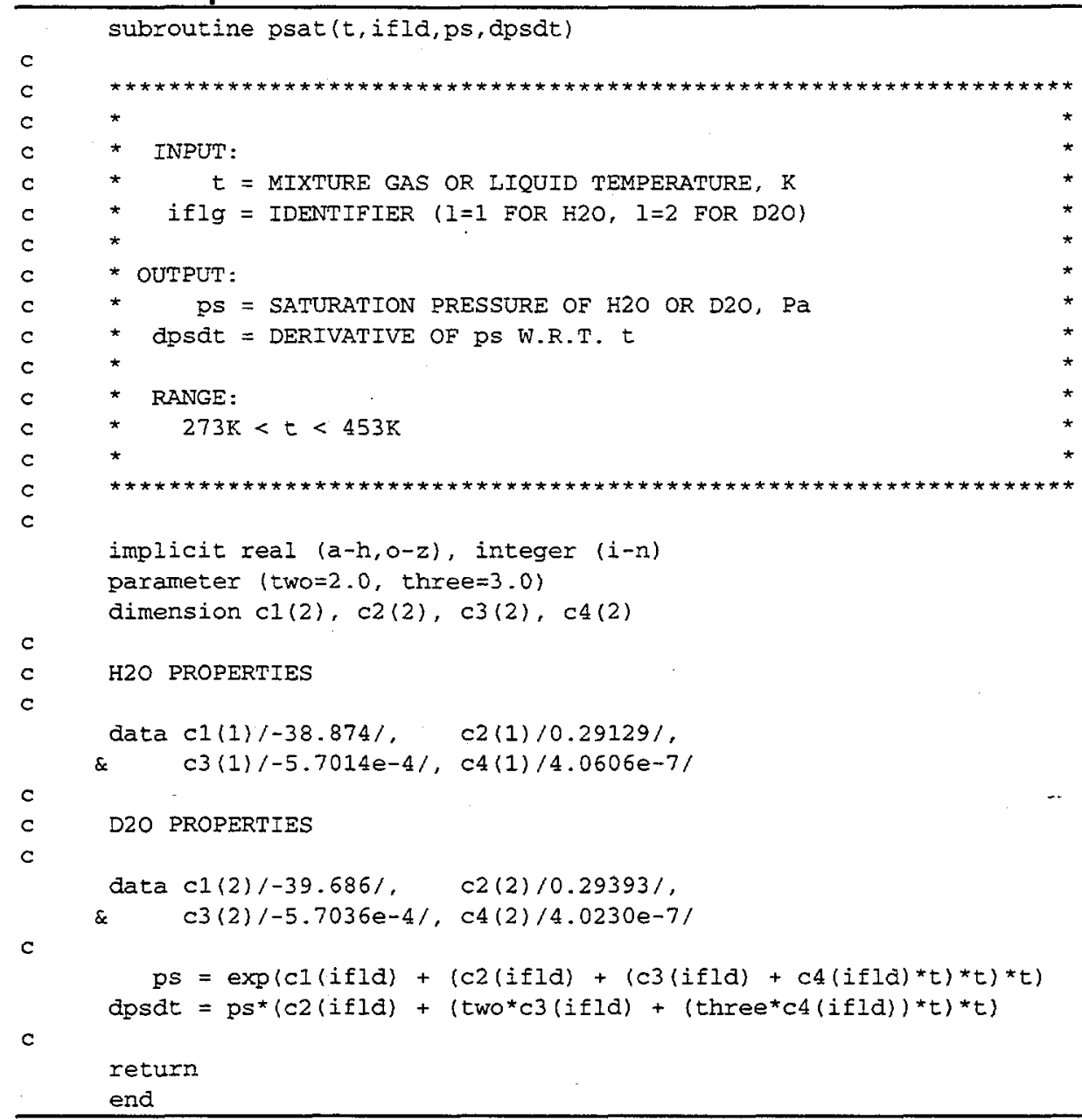

\section{Subroutine pump:}

subroutine pump(flow, dlegi, dlego, diami, diamo,zlil,zlol, $\& \quad z i i 2, z i o 2$, elvi, elvo,elvps,

\& kti1,kto1,kti2,kto2,

\& kvalvei1, kvalvei2, kvalveo1, kvalveo2,

\& kebowi1, kebowo1, kebowi2, kebowo2,

\& rough, temp, omega1, omega2, pdis, delp.

\& ps1,ps2, iout1)

$c$

$c$

$c$

c

c

$c$

c

c

c

c

$c$

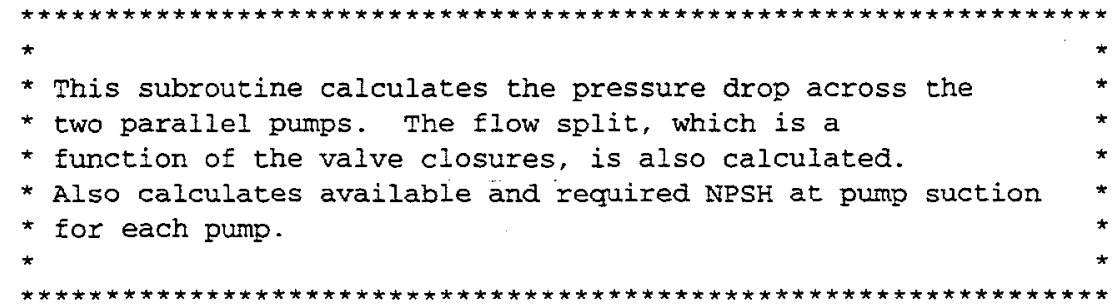

implicit real $(a-h, o-z)$, integer $(i-n)$

real ktil, ktol, kti2, kto2, kvalvel, kvalve2

real ktilp, ktolp, kti2p, kto2p

real kvalveil, kvalvei2, kvalveol, kvalveo2 
real kebowi1, kebowo1, kebowi2, kebowo2

parameter (zero $=0.0$, half $=0.5$, one $=1.0$, four $=4.0$,

$\&$

eight $=8.0$ )

data cf1, cf2, cf3, cf4/.48954, -.71606, .36098,- $-.13510 /$

INPUT :

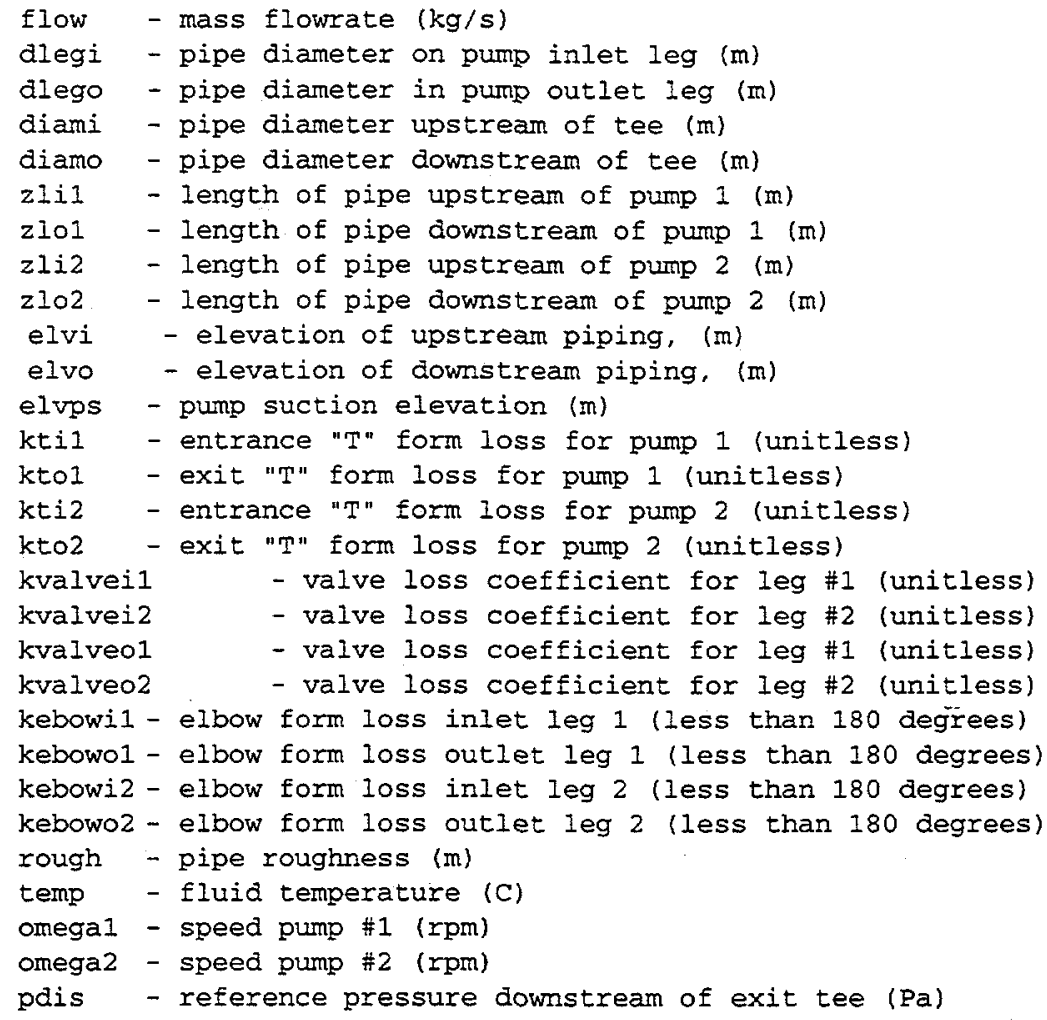

OUTPUT:

delp - pressure drop across entire unit (Pa)

p_conv $=14.696 / 101325.0$

pi $=\operatorname{acos}(-o n e)$

g $\quad=9.807$

conv $=1.0 \mathrm{e}-4$

eps $=1.0 \mathrm{e}-3$

maxit $=1000$

dflow $1=$ zero

iout $4=10$

kvalvel = kvalveil + kvalveol

kvalve2 = kvalvei2 + kvalveo2

$c$

c calculate contraction form loss coefficient for $20^{\text {" to }} 16^{\text {" pipe: }}$

$c$

cc. fmcont $=0.42 *(1.0-(($ dlegi $/$ diami $) * 2))$

c

ar $=($ dlegi $/$ diami $) * * 2$

fmcont $=c f 1+a r^{*}\left(c f 2+a x^{*}\left(c f 3+a{ }^{*} \operatorname{cf} 4\right)\right)$

$c$

write (iout4,701) fmcont

701 format $(1 \mathrm{x}, ' \mathrm{~K}$ contraction $=', 916.8)$ 
write (iout 4,901)

write (iout 4,799 )

write (iout4,901)

799 format (' kti

$k t i l p=k t i l$

$k t i 2 p=k t i 2$

$k t o l p=k t o l$

$k t \circ 2 p=k t o 2$

$c$

c Calculate fluid properties and initial guess for flow split

$c$ base on internal form loss settings.

call propw (temp, den, vis)

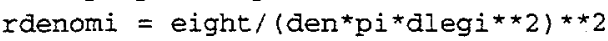

rdenomo $=$ eight $/\left(\right.$ den $* p^{*}$ dlego $\left.* \star 2\right) * \star 2$

$c$

rdenomp $=$ eight $/(\operatorname{den} * p i \star$ diami $* \star 2) \star \star 2$

if (kvalve1.lt.eps .and. kvalve2.It.eps) then

flowl = half

flow2 = half

else

formI = kebowil + kvalve1 + kebowol + Emcont

form2 = kebowi2 + kvalve2 + kebowo $2+$ fmcont

flowl $=($ forml $/($ form $1+$ form 2$)) *$ flow

flow2 $=$ flow - flow

endif

$c$

c Start the iterative loop:

$c$

do iter=1, maxit

C $===$ Calculate function at current iterate \& perturbation $=========$

c Calculate perturbed flows

flowlp $=$ Elowl* (one+eps)

flow $2 p=$ Elow - flow $1 p$

c calculate the "T" form loss coefficients and write results:

c ( if these expressions are commented out, constant input values are used,

$c$ at current flow rate:

$k t i 1=0.99 *($ flow $/$ flow 1$) * * 2)-0.82 *($ flow $/$ flow 1$)+1.02$

$k t i 2=(0.65 *((($ flowl $/$ flow $)-0.22) * * 2)-0.03) *(($ flow $/$ flow $) * * 2)$

ktol $=1.19 *($ flow $/$ flow $) * * 2)-$

$\& \quad 1.73 *(($ flow $2 *$ flow $) /($ flow $1 * * 2))+$

$\& \quad 1.34 *($ flow $2 /$ flow $) * * 2)$

$k \operatorname{to} 2=1.19 *($ flow $/$ flow 2$) * * 2)-$

\& $\quad 1.73 *(($ flow $1 *$ flow $) /($ flow $2 * 2))+$

$\& \quad 1.34 *(($ flow $1 /$ flow 2$) * * 2)$

$c$

$c$ at perturbed flow rate:

ktilp $=0.99 *($ flow $/$ flowlp $) * 2)-0.82 *($ flow $/$ flowlp $)+1.02$

$k t i 2 p=(0.65 *(($ flowlp $/$ flow $)-0.22) * * 2)-0.03) *(($ flow $/$ Elow $2 p) * * 2)$

ktolp $=1.19 *(($ Elow $/$ Elowlp $) * * 2)$ -

$\& \quad 1.73 *(($ Elow2p*flow $) /($ Elow1p**2))+

$\& \quad 1.34 *($ flow $2 p /$ flowlp $) * * 2)$

kto $2 p=1.19 *(($ flow $/$ flow $2 p) * * 2)-$

\& $\quad 1.73 *(($ flowlp* flow $) /($ flow $2 p * \star 2))+$

$\& \quad 1.34 *($ (flow1p/Elow $2 \mathrm{p}) * * 2)$

$c$ 


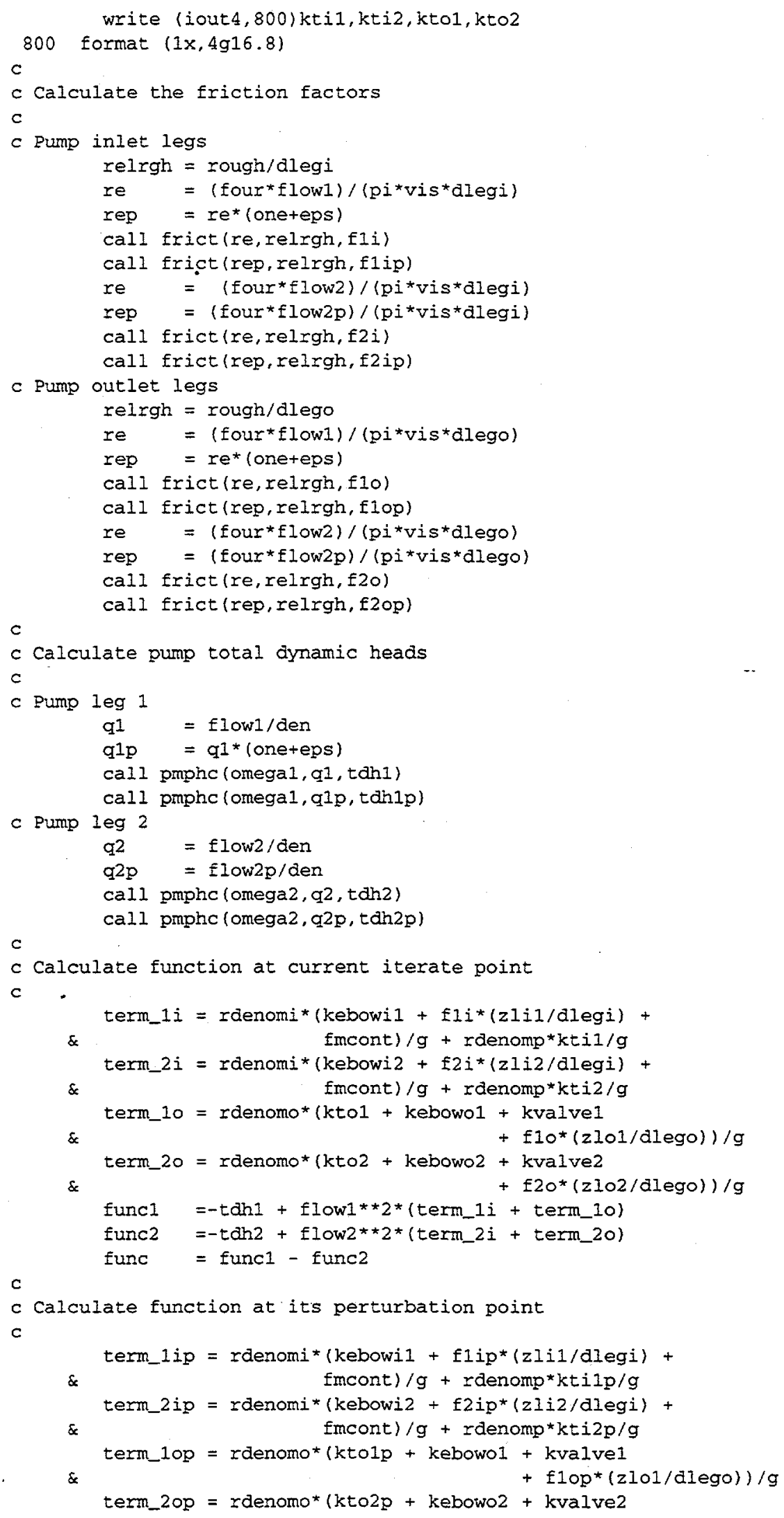




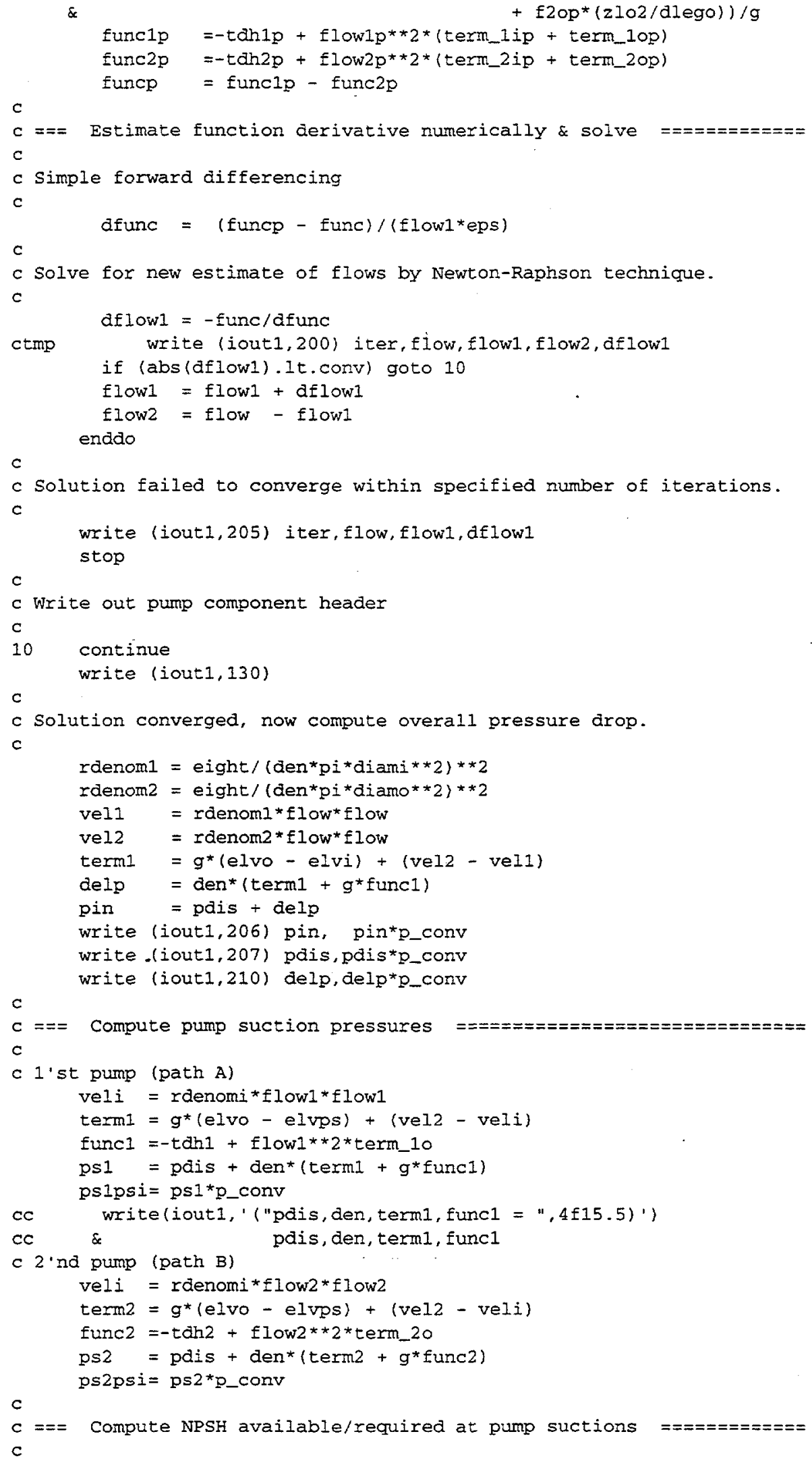

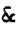




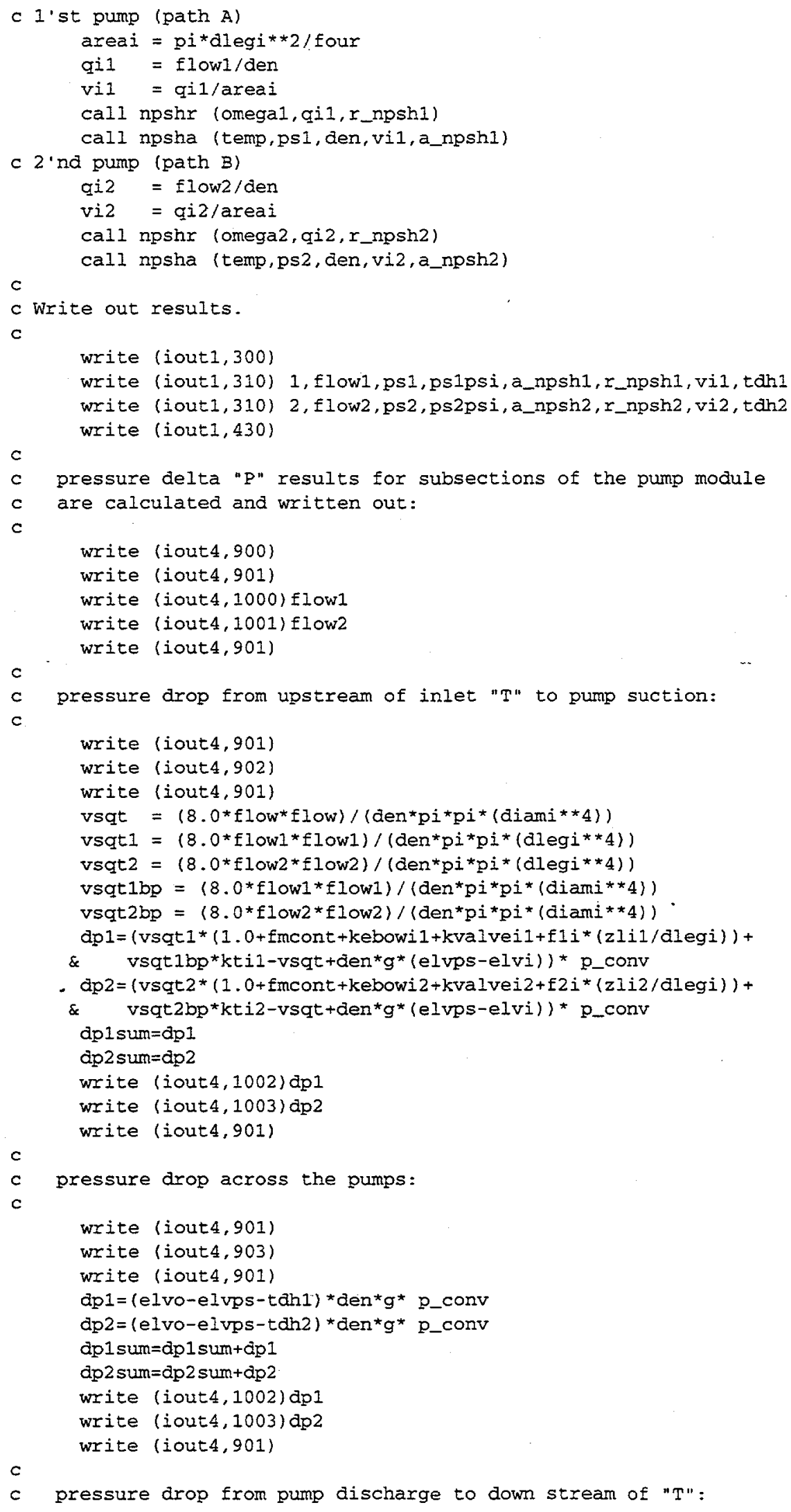


c

write (iout4,901)

write (iout4,904)

write (iout 4,901 )

vsqt $=\left(8.0 *\right.$ flow ${ }^{*}$ low $) /($ den $* \mathrm{pi} * \mathrm{pi} *($ diamo**4)$)$

vsqt $1=(8.0 *$ flow $1 *$ flow 1$) /($ den*pi*pi* (dlego**4)$)$

vsqt $2=(8.0 *$ flow $2 *$ flow 2$) /($ den $* \mathrm{p} * \mathrm{p} i *($ dlego $* * 4))$

$\mathrm{dp} 1=\left(\right.$ vsqt $1 *\left(\right.$ kebowo $1+\mathrm{kval}$ veo $1+\mathrm{kto} 1+\mathrm{f} 10^{*}(z 101 / \mathrm{d} 1$ ego $\left.)-1.0\right)+$

$\&$ vsqt $) * p_{-}$conv

dp $2=($ vsqt $2 *($ kebowo $2+$ kvalveo $2+$ kto $2+f 20 *(z l o 2 /$ dlego $)-1.0)+$

$\&$ vsqt) * $p_{-}$conv

dp1 sum=dp1 sum $+d p 1$

$\mathrm{dp} 2 \mathrm{sum}=\mathrm{dp} 2 \mathrm{sum}+\mathrm{dp} 2$

write (iout4,1002) dp1

write (iout 4,1003 ) do2

write (iout4,901)

write (iout4, 1004) dp1sum

write (iout 4,1005 ) do2sum

write (iout 4,901 )

dprtn $=$ delp*p_conv

write (iout4,1006) dprtn

c

$c$

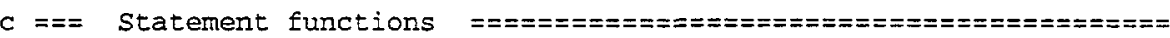

130 format $/$

$\&^{\prime}====================$ START OF PUMP COMPONENT RESULTS $====1$,

$\& \&^{\prime}==================1, /$ )

200 Eormat (' iter, flow, flow1, flow2, dflow1 = ',i5, 4f10.3)

205 format' failed to converge', /1,

\& 'iter, flow, flowl, dflow $=1, i 5,3 \pm 10.3$

206 format('Pressure upstream of inlet tee $=$ ', 112.1 ,

$\& \quad \operatorname{Pa}(1, f 10.3, '$ psia)')

207 format('Pressure downstream of exit tee $=$ ', £12.1, $\& \quad \operatorname{Pa}(', \pm 10.3, '$ psia)')

210 format ('Purmp component pressure arop = ', 12.1 , \& 'Pa (',f10.3,' psid)')

300 format $/ /$,

\&. pump flow

\&. velocity

\&. id..

suction

suction

NPSH

NPSH '

$\&$.

$\&^{\circ} \quad(\mathrm{kg} / \mathrm{s})$

TDH', /,

pressure

pressure available required',

$(\mathrm{m} / \mathrm{s})$

(Pa)

(psia)

(m)

(m)

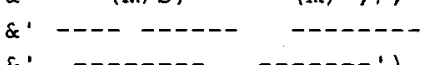

310 format $(13, \pm 9.1,6 \pm 11.1)$

430 format (/,

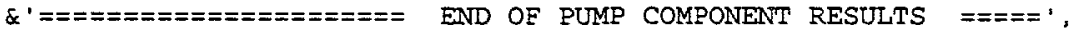

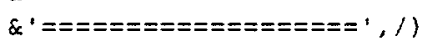

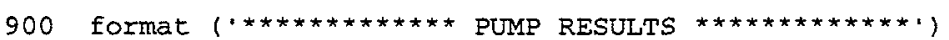

901 format (' ')

902 format ( $" \star \star \star$ PRESS DRP UPSTM "T' - PUMP SUCTION $\star \star \star *)$

903 format $(\cdot \star \star \star$ PRESS DRP ACROSS PUMPS $\star \star \star 1)$

904 format ( $* * \star$ PRESS DRP PUMP DSCH - DNSTRM "T" $* \star *$ )

1000 format ( $1 x$, PUMP \#1 mass flowrate $=, .914 .8, \cdot \mathrm{kg} / \mathrm{s}$ ')

1001 format $\left(1 \mathrm{x}\right.$, 'PUMP \#2 mass flowrate $=., 914.8,{ }^{\prime} \mathrm{kg} / \mathrm{s}^{\prime}$ )

1002 format $(1 x$, 'delta $P$ (sys $\# 1)=$ 'g14.7,' psid')

1003 format $(1 \mathrm{x}$, 'delta $\mathrm{P}$ (sys \#2) = 'g14.7,' psid')

1004 format ( $1 x$, ' total Delta $P$ \#1 =',g14.7,' psid')

1005 format ( $1 x$, ' total Delta $P \# 2=\cdot$ g14.7,' psid') 


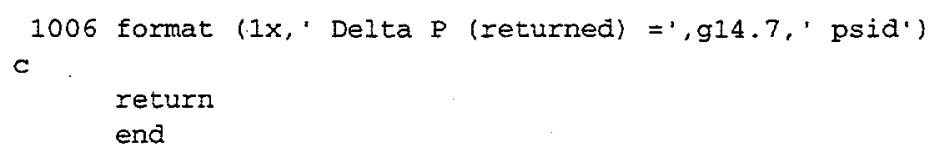

\section{Input File: (input.dat)}

20 " base piping with 16" piping within tees QRTRAC input file:

This is the input deck for a model of the APT target/blanket outer loop from the outlet header to the inlet header. Units are SI. section \#1: (pressurizer to inlet header)
zlngth length of pipe (m)
diam pipe dianeter (m)
rough pipe roughness $(\mathrm{m})$
dhdrin inlet header diameter
Emeb elbow loss coefficient
elbnum number of elbows
(0 no, 1 yes)
flow mass flowrate
temp fluid temperature
pout outlet pressure

delz elevation change (outlet - inlet)

Ement entrance form loss coefficient

fmext exit form loss coefficient

isolve flag option for solving supply \& demand curves

\begin{tabular}{|c|c|c|c|c|}
\hline zlngth & diam & rough & delz & dharin \\
\hline 31.624 & .47788 & $5.0 e-5$ & -.324 & 0.736 \\
\hline fment & fmext & fmeb & elbnum & \\
\hline 0.0 & 0.335 & 0.156 & 6.0 & \\
\hline isolve & flow & phdin & (design & goal $=1569.01$ \\
\hline 0 & 1569.0 & $1.401 \mathrm{e}$ & & \\
\hline
\end{tabular}

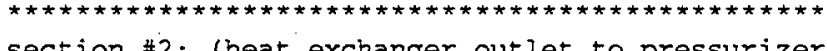

zlngth length of pipe

diam pipe diameter

delz elevation change (outlet - inlet)

fment entrance form loss coefficient

fmext exit form loss coefficient

- fmeb elbow loss coefficient

elbnum number of elbows

\begin{tabular}{|c|c|c|c|}
\hline zlngth & diam & delz & \\
\hline 0.0 & .47788 & 0.0 & \\
\hline fment & fmext & fmeb & elbnum \\
\hline 0.0 & 0.0 & 0.0 & 0.0 \\
\hline
\end{tabular}

section \#3: (heat exchangers)

zli1 entrance side pipe length for leg 1

zlo1 exit side pipe length for leg 1

zli2 entrance side pipe length for leg 2

2102 exit side pipe. length for leg 2

zltub tube length

delzhx elevation change (out - in)

aleg pipe diameter

diat tube diameter

rght tube roughness

tuben number of tubes

fmhxtil - inlet "T" loss leg 1

fmhxto1 - outlet " $T$ " loss leg 1 
fmhxti2 - inlet "T" loss leg 2

fmbxto2 - outlet "T" loss leg 2

fmhi inlet header entrance form loss coefficient

fmho outlet header exit form lass coefficient

fmti tube entrance form loss coefficient

fmto tube exit form loss coefficient

fmeb elbow loss coefficient

elbup1 number of elbows on leg 1 (upstream)

elbup2 number of elbows on leg 2 (upstream)

elbdnl number of elbows on leg 1 (down stream)

elban2 number of elbows on leg 2 (down stream)

vivi valve loss leg \#1

vlv2 valve loss leg \#2

tin entrance fluid temperature

tout exit fluid temperature

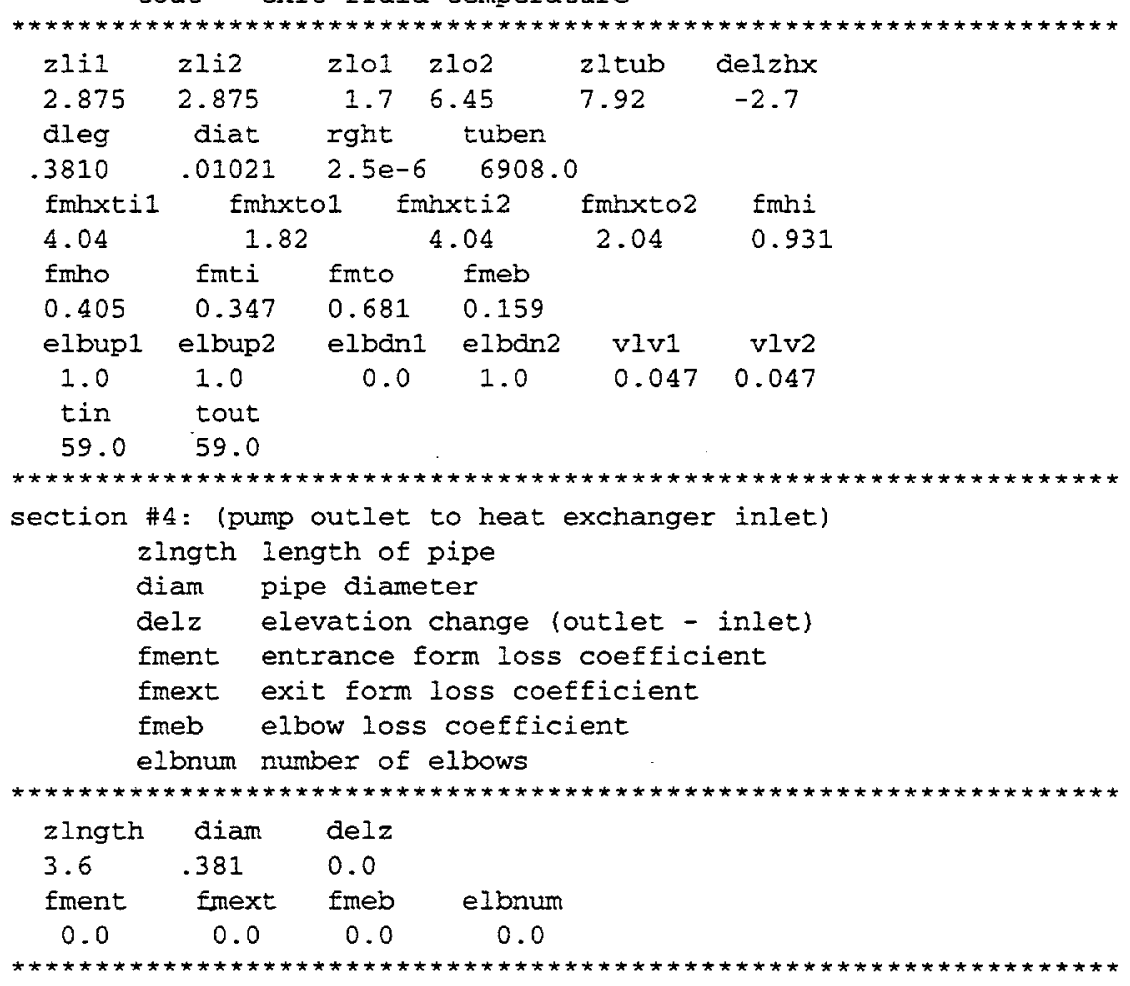

section \#5: (pumps)

ziil entrance side pipe length pump 1

zlo1 exit side pipe length pump 1

zli2 entrance side pipe length pump 2

zlo2 exit side pipe length pump 2

dlegi entrance side diameter

dlego exit side diameter

fmltil entrance " $T$ " form loss coefficient for leg 1

fmltol exit " $T$ " form loss coefficient for leg 1

fmlti2 entrance "T" form loss coefficient for leg 2

fmito2 exit "T" form loss coefficient for leg 2

fmeb5il inlet elbow loss coefficient for leg 1

fmeb5ol outlet elbow loss coefficient for leg 1

fmeb5i2 inlet elbow loss coefficient for leg 2

fmeb502 outlet elbow loss coefficient for leg 2

zkvlvil inlet leg \#1 valve loss coefficient

zkvlvi2 inlet leg \#2 valve loss coefficient

zkvlvol outlet leg \#1 valve loss coefficient

zkvlvo2 outlet leg \#2 valve loss coefficient 
omegal pump \#1 angular velocity

omega2 pump \#2 angular velocity

elvi inlet elevation

elvo outlet elevation

elvps pump suction elevation

\begin{tabular}{|c|c|c|c|c|c|}
\hline zIil & 2101 & $z 1 i 2$ & 2102 & dlegi & alego \\
\hline 2.2 & 2.875 & 6.95 & 2.875 & 0.3810 & 0.3810 \\
\hline fmItil & fmltol & fmlti2 & fmlto2 & & \\
\hline 3.34 & 2.64 & 0.084 & 2.64 & & \\
\hline fmeb5i1 & fmeb5ol & fmeb5i2 & fmeb5o2 & & \\
\hline 0.0 & 0.159 & 0.159 & 0.15 & 59 & \\
\hline zkvlvi1 & zkvlvi2 & zkvlvol & zkvlvo2 & omega 1 & omega2 \\
\hline 0.047 & 0.047 & 0.547 & 0.547 & 665.0 & 665.0 \\
\hline elvi & elvo & elvps & & & \\
\hline 0.0 & 2.7 & 2.2 & & & \\
\hline
\end{tabular}

section \#6: (outlet header to pump inlet)

zingth length of pipe

diam pipe diameter

delz elevation change (outlet - inlet)

dhdrot outlet header diameter

fment entrance form loss coefficient

fmext exit form loss coefficient

fmeb elbow loss coefficient

elbnum number of elbows

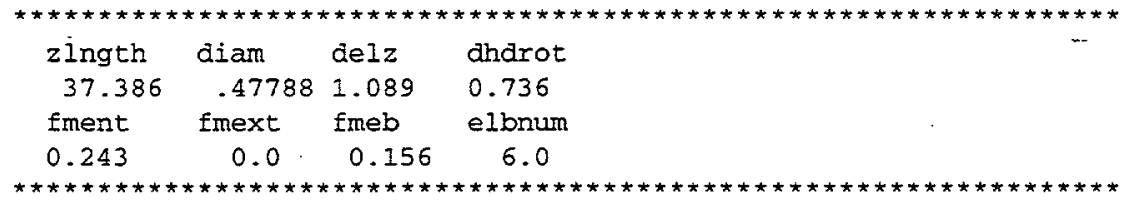

Modules: (inlet header to outlet header for each module)

c nmods - number of modules connectec to fixed headers

c kent - Entrance loss coefficient at inlet header (unitless)

c kext - Exit loss coefficient at outlet header (unitless)

c kform_i - inlet segment form losses (unitless)

c kform_h - outlet segment form losses (unitless)

c kform_o- heated segment form losses (unitless)

c kvalv_i - valve loss coefficient for inlet segment (unitless)

c kvalv_h - valve loss coefficient for inlet segment (unitless)

c kvalv_o - valve loss coefficient for inlet segment (unitless)

c kebow_i - elbow form loss inlet leg (less than 180 degrees)

c kebow_h - elbow form loss heated leg (less than 180 degrees)

c kebow_o - elbow form loss outlet leg (less than 180 degrees)

c nebow_i - number of elbows in inlet leg (unitless)

c nebow_h - number of elbows in heated leg (unitless)

c nebow_o - number of elbows in outlet leg (unitless)

c dleg_i - pipe diameter on inlet leg (m)

c dleg_h - pipe diameter on heated leg (m)

c dleg_o - pipe diameter in outlet leg (m)

c elv_a - elevation of entrance to heated segment (m)

$c$ elv_b - elevation of exit to heated segment (m)

c $\quad z I_{-} i$ - Length of piping in inlet segment (m)

c $\quad z I_{m}$ - Length of piping in heated segment (m)

c $z z_{*}$ - Length of piping in outlet segment (m)

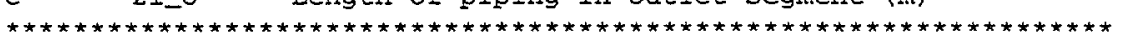
nmods

5

MODULE 1:

$\begin{array}{ll}\text { kent } & \text { kext } \\ 1.0 & 1.0\end{array}$


kform_i

0.3

kvalv_i 0.0

kebow_i

0.2

nebow i

3

dleg_i

0.25

elv_a

0.0

21_i

MODULE 2:

$$
4.0
$$

$$
\text { kent }
$$

1.0

kform_i

0.3

kvalv_i

0.0

kebow_i

0.2

nebow_i 3

dleg_i

0.2032

elv_a

0.0

21_i

MODULE 3:

$$
4.0
$$

kent

1.0

kform_i

0.3

kvalv_i

0.0

kebow_i

0.2

nebow_i

3

0.18

elv_a

$0 . \overline{0}$

zI_i

MODULE 4:

kent

1.0

kform_i

0.3

kvalv_i

0.0

kebow_i 0.2

nebow_i

3

dleg_i

0.15

elv_a kform_h

0.0

kvalv_h

0.0

kebow_h

0.2

nebow_h

2

dleg_h

0.25

elv_b

0.0

zl_h

6.0

kext

1.0

kform_h

0.0

kvalv_h

0.0

kebow_h

0.2

nebow_h

2

dleg_h

0.2032

elv_b

0.0

2I_h
6.0

kext

1.0

kform_h

0.0

kvalv_h

0.0

kebow_h

0.2

nebow_h

2

dleg_h

0.18

elv_b

0.0

zl_h

6.0

kext

1.0

kform_h

$$
0.0
$$

kvalv_h

0.0

kebow_h

0.2

nebow_h

2

dleg_h

0.15

elv_b kform_o

0.3

kvalv_o

$$
0.0
$$

kebow_o

0.2

nebow_o

$$
3
$$

dleg_o

0.25

$$
\text { z1_o }
$$

4.0

kform_o

0.3

kvalv_o

0.0

kebow_o

0.2

nebow_o

3

dleg_o

0.2032

zl_o
4.0

kform_o

0.3

kvalv_o

0.0

kebow_o

0.2

nebow_o

3

dleg_o

0.18

21_o

4.0

kform_o

0.3

kvalv_o

0.0

kebow_o

0.2

nebow_o

3

dleg_o

0.15 


$\begin{array}{rrr}0.0 & 0.0 & \\ z I_{-} i & z l_{-} h & z 1 \_0 \\ 4.0 & 6.0 & 4.0\end{array}$

MODULE 5:

$\begin{array}{ccc}\text { kent } & \text { kext } & \\ 1.0 & 1.0 & \\ \text { kform_i } & \text { kform_h } & \text { kform_o } \\ 0.3 & 0.0 & 0.3 \\ \text { kvalv_i } & \text { kvalv_h } & \text { kvalv_o } \\ 0.0 & 0.0 & 0.0 \\ \text { kebow_i } & \text { kebow_h } & \text { kebow_o } \\ 0.2 & 0.2 & 0.2 \\ \text { nebow_i } & \text { nebow_h } & \text { nebow_o } \\ 3 & 2 & 3 \\ \text { dleg_i } & \text { aleg_h } & \text { dleg_o } \\ 0.13 & 0.13 & 0.13 \\ \text { elv_a } & \text { elv_b } & \\ 0.0 & 0.0 & \\ \text { zl_i } & \text { zl_h } & \text { zl_o } \\ 4.0 & 6.0 & 4.0\end{array}$

Output File: (results.out)

20 " base piping with 16 " piping within tees

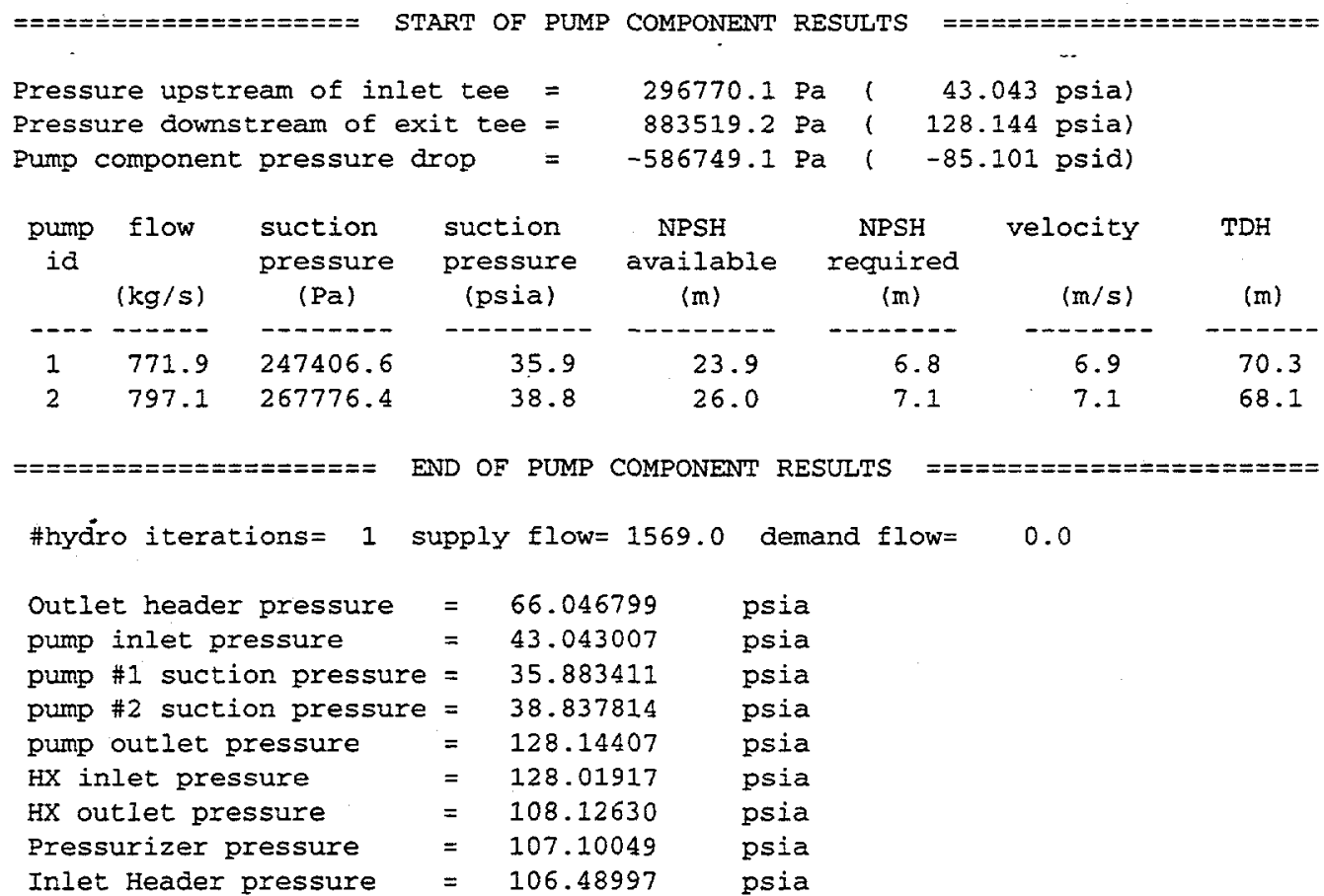


(This Page Intentionally Left Blank) 


\section{Appendix D: RHRTRC - A Simple Hydraulic Code for RHR Assessment}

This appendix contains the source listing for the code named RHRTRC and all of its associated subroutines. RHRTRC was written specifically for assessing the steadystate isothermal hydraulic behavior of the RHR coolant loop. Sample input and output files are provided.

\section{Program rhrtrc:}

program rhrtrc

c This program calculates the pressure profile in the outer

c flow loop of the APT target/blanket RHR cooling system. The

c mass flowrate and the pressure at the inlet header are

c specified, and the following pressures are calculated

c sequentially: heat exchanger outlet, heat

c exchanger inlet, pump outlet, pump inlet, and the outlet

c header.

$c$

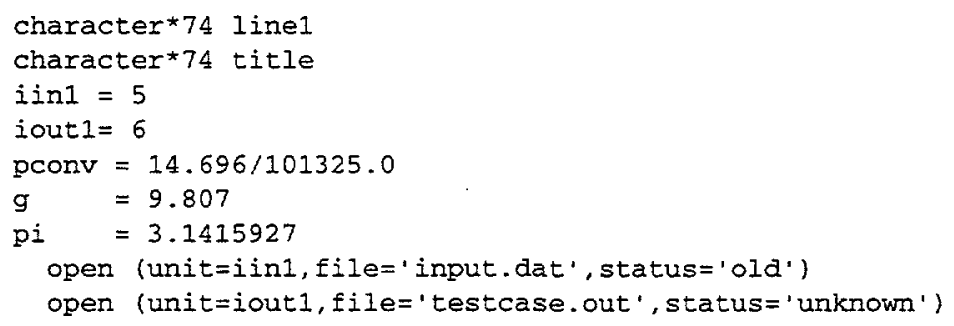


linel $=1$ ine1

read (iin1, *) zlg1, diam1, rough, delz1, dharin

write (iout1,*)zlg1, diam1, rough, delz1, dhdrin

read (iin1, 900)linel

write (iout1,900)linel

read (iinl, *) fment 1, fmext1, fmebl, elbn1, ckv1v1, vlvcf1

write (iout 1, *) fment1, fmext1, fmebl, elbn1, ckvlv1, vlvcfl

read (iin 1,900$)$ line1

write (iout 1,900 ) line 1

read (iinl, *) flow1, pout, flmult

write (iout $1,{ }^{*}$ ) flowl, pout, flmult

$c$

$c$

c

c

c

$\mathrm{C}$

$c$

c

$c$

c

c

$c$

c

section \#2 (heat exchanger)

zltub tube length

delahx change in elevation (out - in)

diatb tube diameter

rght tube roughness

tuben number of tubes in a heat exchanger

fmhai inlet header loss

fmhdo outlet header loss

fmtbi inlet tube loss

fintbo outlet tube loss

tmphxi $h x$ inlet side temp. (input not used/calculated)

tmphxo heat exchanger outlet side temperature

do $12 i=1,14$

read (iin 1,900$)$ linel

$$
\text { continue }
$$

write (iout1, 2003)

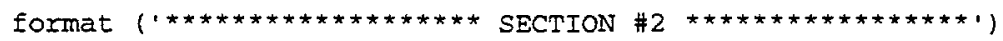

read (inl, 900) linel

write (iout 1,900 ) linel

read (iinl,*)zltub, delzhx

write (iout $1, *) z l t u b$, delzhx

read (iinl, 900) Iinel

write (iout 1,900$)$ linel

read (iinl,*) diatb, rght, tuben

write (iout $1,{ }^{*}$ ) diatb, rght, tuben

read (iin 1,900$)$ linel

wxite (iout 1,900 ) linel

read (iinl, *) fmindi, fmhdo, fmtbi, fmtbo

write (iout $1, *$ ) fmhdi, fmhdo, fmtbi, fntbo

read (iin1, 900) linel

write (iout 1,900 ) linel

read (iin1,*) tmphxi, tmphxo

write (iout $1, *$ ) tmphxi, tmphxo

section \#3 (pump to heat exchanger)

zlg3 length

delz3 delta " $z$ "

fment 3 entrance loss coef.

fmext3 exit loss coef.

fmeb3 elbow loss coef.

elbn3 number of elbows

ckvlv3 check valve form loss coefficient

vivcf3 valve form loss coefficient

do $13 i=1,11$

read $($ in 1,900$)$ linel 


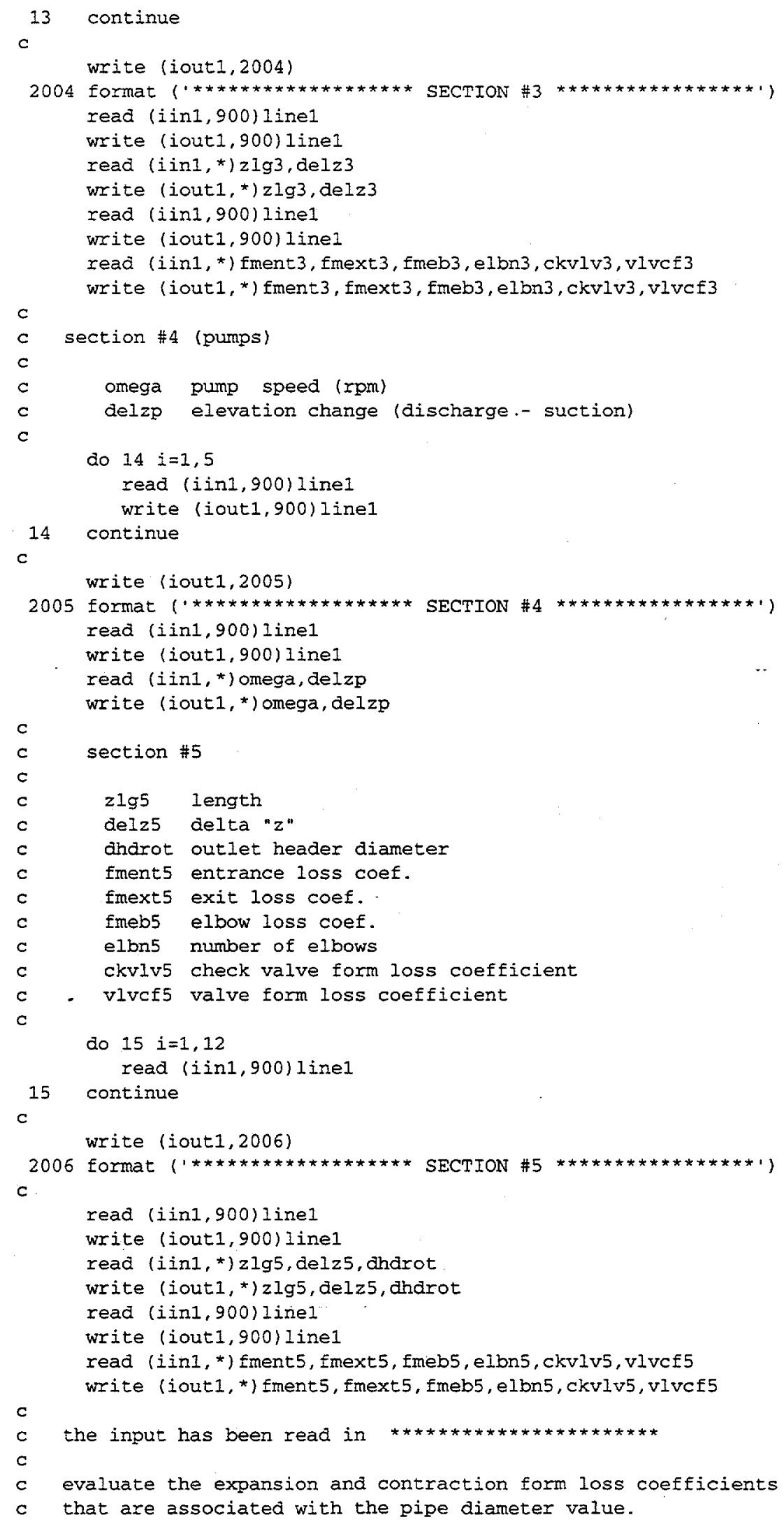


c

dhxhdr $=0.406$

call kloss (diaml, ahdrin, dhxhar, fpipex, fpipin, fhxin, fhxot)

fmext 1 = fpipex

fmhdi $=$ Ehxin

Enhdo $=$ fhxot

fmext5 = fpipin

write (iout 1,1010 )

write (iout 1,1100 )

write (iout 1, *) fmext1, fmidi, Emhdo, fmext5

write (iout 1,1010 )

c

$c$

$c$

c

valuate the flowrate and hx inlet temperature. At 1\% full flow the delta "T" across the heat exchanger is 16 deg $C$.

flow $=$ flmult*Elowl

delt $1 p=16.0$

tmphxi = tmphxo+deltlp/flmult

c

c

write (iout 1, 1010)

write (iout1,998) Elmult

write (iout1,999) tmphxi

write (iout1, 1010)

c

c

c

- fmhdi, fmhdo, fmtbi, fmtbo

\& tuben, aiatb, rght, tmphxi, tmphxo, delp)

phxin=phxout + delp

call pipdlp (flow, diaml, diam1, dharin, zlg1, delz1, rough.

fmebl, elbnl, fment 1, fmext 1

phxout $=$ pout + delp
C

c

$c$

calculate the pressure drop in section \#3

call pipdlp (Elow, diam1, diam1, diam1, zlg3, delz3, rough,

\&.

\&

ppmpot $=$ phxin + delp

$c$

calculate the pressure drop across the pumps (outlet - inlet)

call propw (tmphxi, den, vis)

vflow $=$ flow/den

call pmphc (omega, vflow, tah)

delp=den * $g$ *tdh

ppmpin=ppmpot-delp + den $* g *$ delzp

$c$

c

calculate the pressure drop in section \#5

call pipalp (flow, diam1, tharot, diaml, zlg5, delz5, rough, 


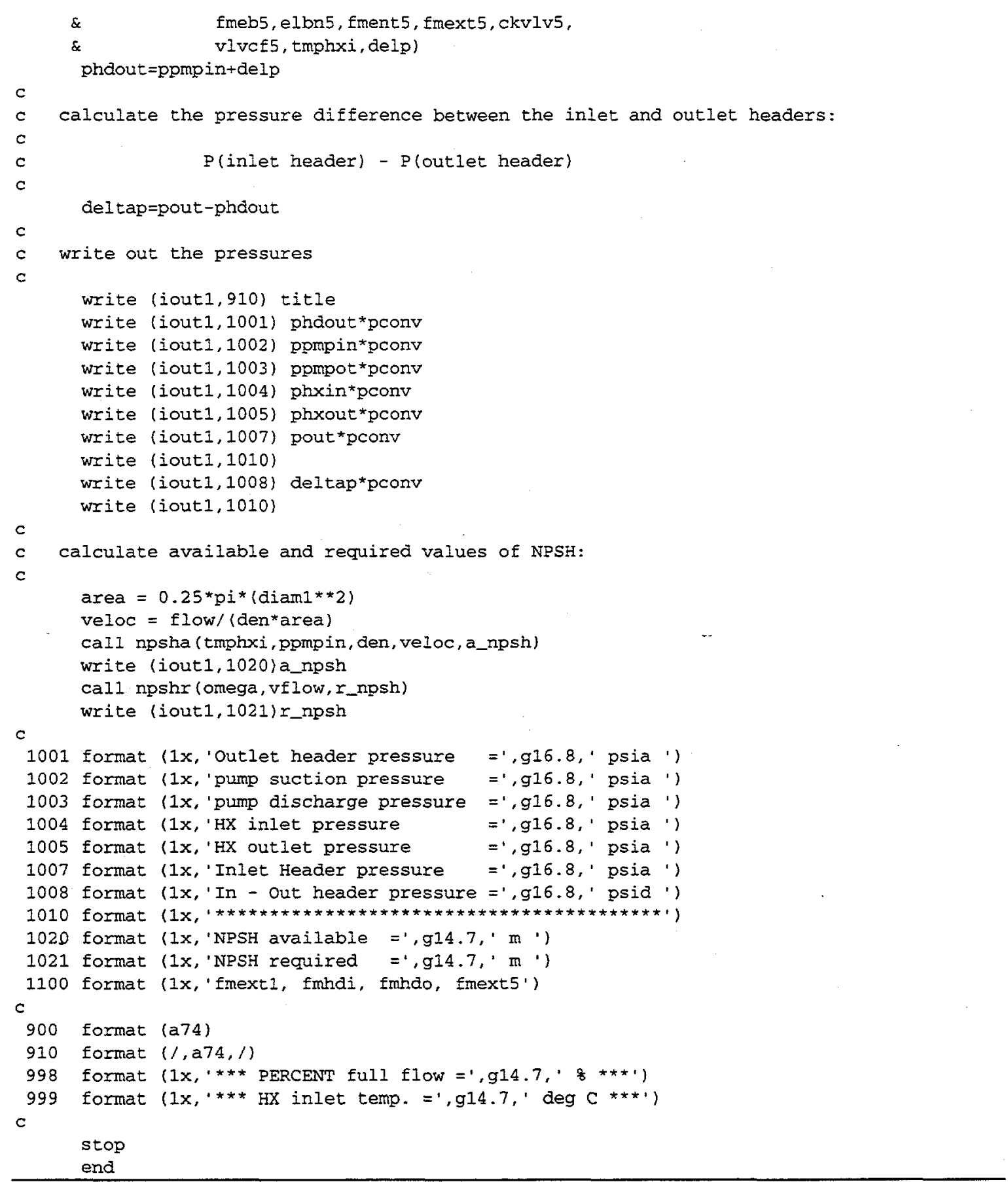

\section{Subroutine frict:}

subroutine frict(re,ed,zf)

This subroutine calculates the Moody friction factor for a

tube. Inputs are the Reynolds \#, and the relative roughness.

The Darcy friction factor is returned.

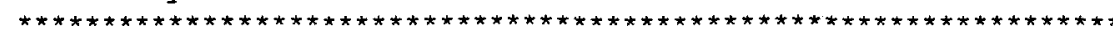


if (re.gt. 50.0 ) go to 10 $r e=50.0$

10 if (re.le. 2300.0 ) then

$z f=64.0 / r e$

else if (re.ge. 4000.0 ) then

$z f=1.0 /\left(\left(1.14-2.0 * a \log 10\left(e^{d}+21.25 /\left(\mathrm{re}^{* *} .9\right)\right)\right) * * 2\right)$

else

$z f=.23158-2.0549 e-4 * r e+5.6351 e-8 *\left(r e^{* *} 2\right)-6.7336 e-12 *\left(r e^{* *} 3\right)$

end if

return

end

\section{Subroutine heatx:}

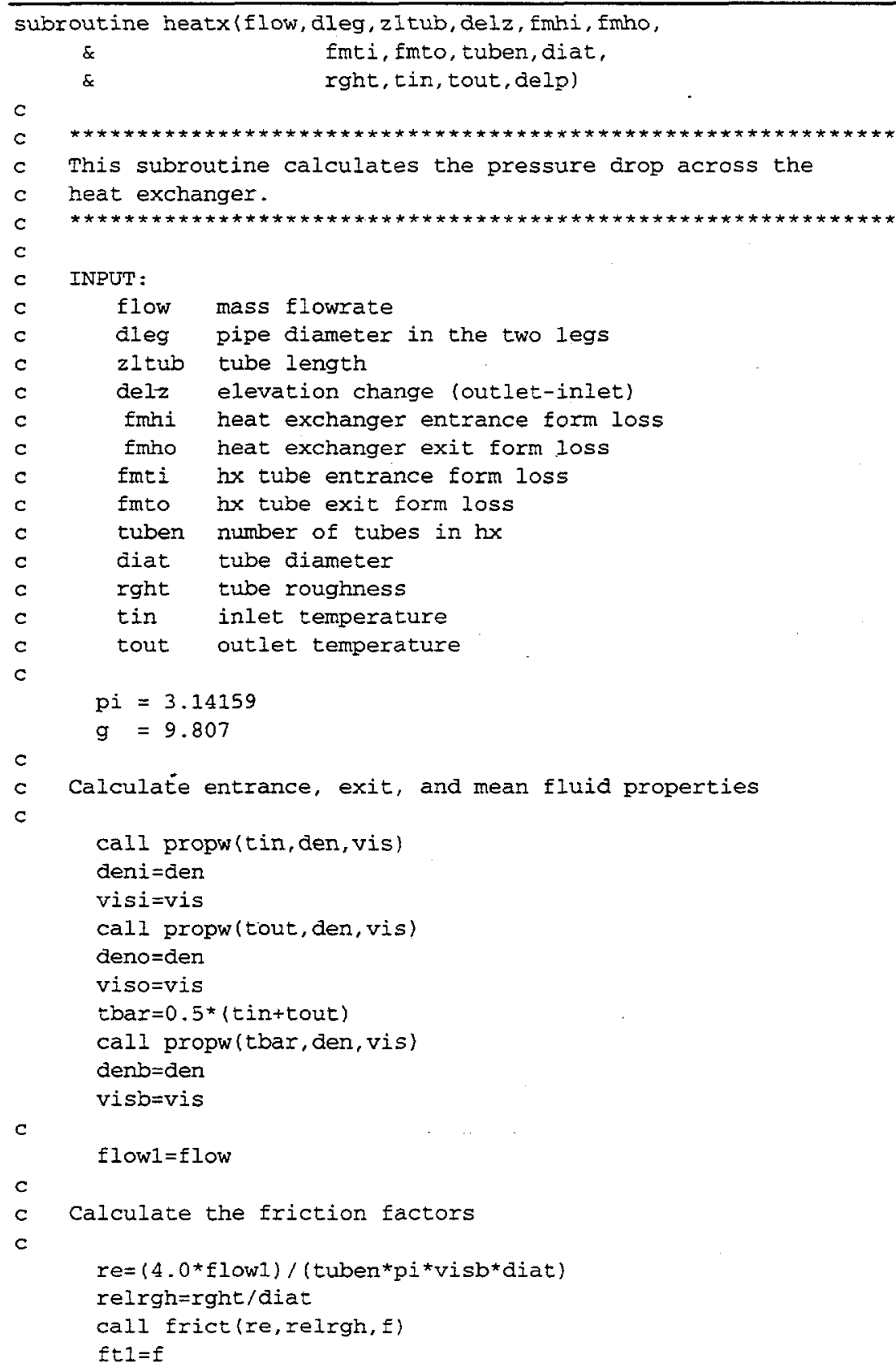


$\mathrm{c}$
$\mathrm{c}$
$\mathrm{c}$

c

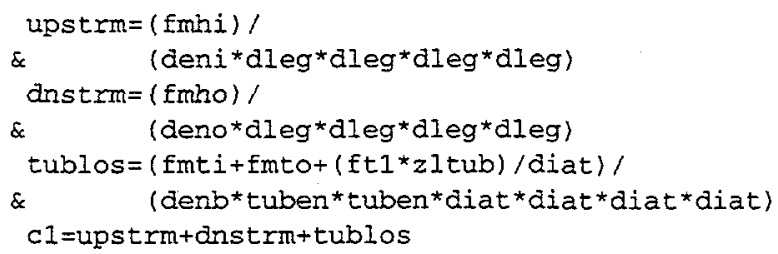

\section{Subroutine kloss:}

subroutine kloss (dpipe, dhdr, dhxhdr,

$$
\text { \& } \quad \text { fpipex, fpipin, fhxin, fhxot) }
$$

c This subroutine evaluates the sudden expansion and

c contraction form loss coefficients for the RHR system,

c that are a function of the pipe diameter.

INPUTS :

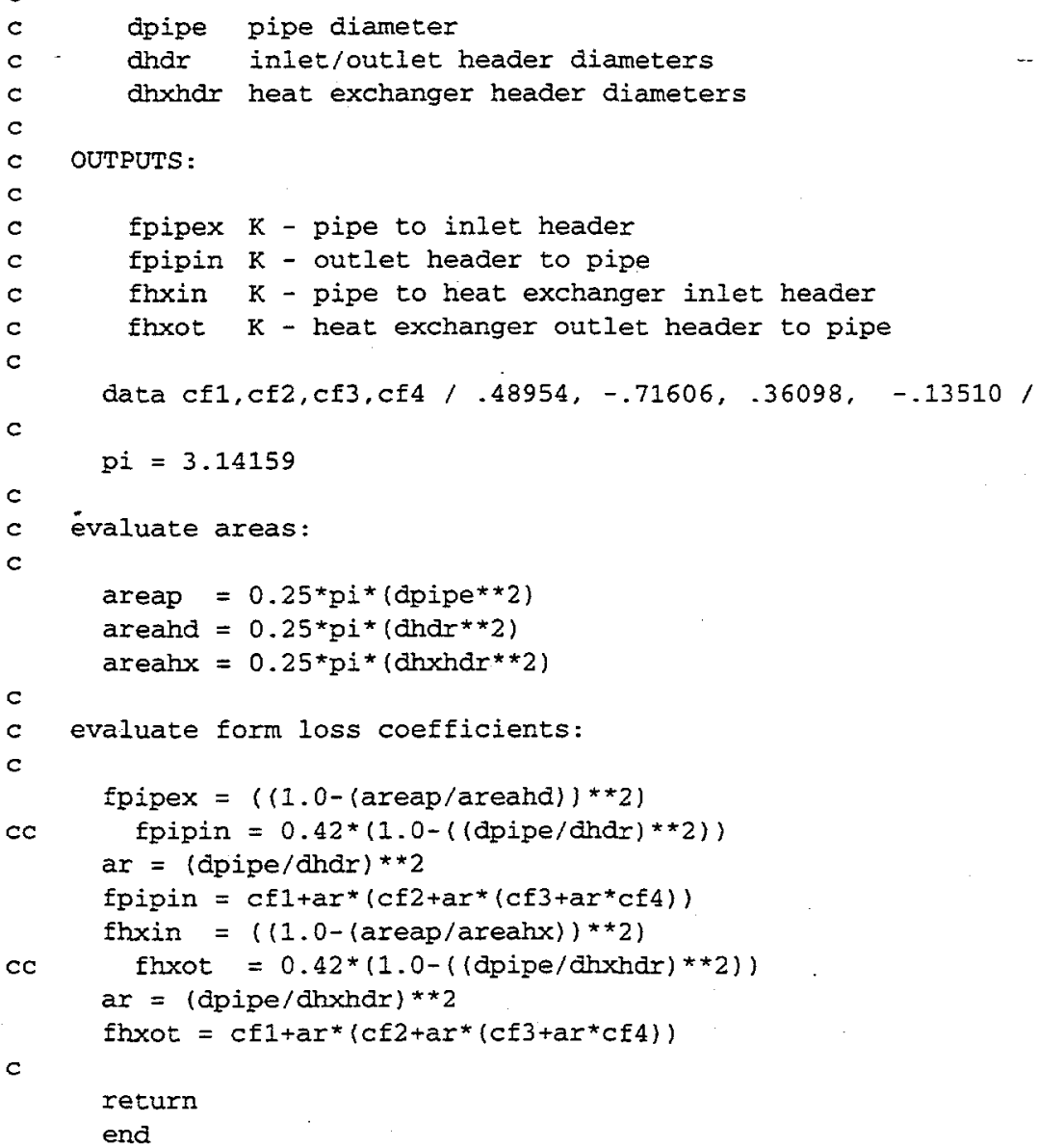

\section{Subroutine npsha:}


subroutine npsha (tfld,pfld, rhof, velf,a_npsh)

c

C $* *$

C $\quad$ *

c

c * INPUT:

c

c

c

$c$

$$
\text { C }
$$

$\mathrm{c}$

c

* This subroutine computes the net positive suction head

* available at the piping location provided.

* tfla - fluia temperature (C)

* pfld - fluid pressure (Pa)

* rhof - fluid density $\left(\mathrm{kg} / \mathrm{m}^{\wedge} 3\right)$

* velf - fluid velocity $(\mathrm{m} / \mathrm{s})$

* outrput

* a_npsh - net positive suction head available (m)

implicit real $(a-h, a-z)$, integer $(i-n)$

parameter (two $=2.0$ )

c Gravitational constant:

data grav / $9.807 /$

c CONVERSION FACTORS EMPLOYED (SI)

c

c con_tmp - converts $C$ to $\mathrm{K}$

c

$$
\text { con_tmp }=273.15
$$

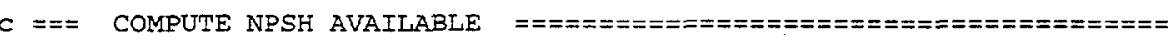

C CONVERT TEMPERATURE TO ABSOLUTE

$t k=t f l d+$ con_tmp

$c$

c COMPUTE FLUID SATURATION PRESSURE FOR H2O

c

ifluid $=1$

call psat (tk, ifluid, pvap, xdum)

c

c EVALUATE NPSH AVAILABLE FUNCTION

c

a_npsh $=(p f l d-p v a p) /\left(g r a v^{\star} r h o f\right)+v e l f /(t w o * g r a v)$

$c$

return

end

\section{Subroutine npshr:}

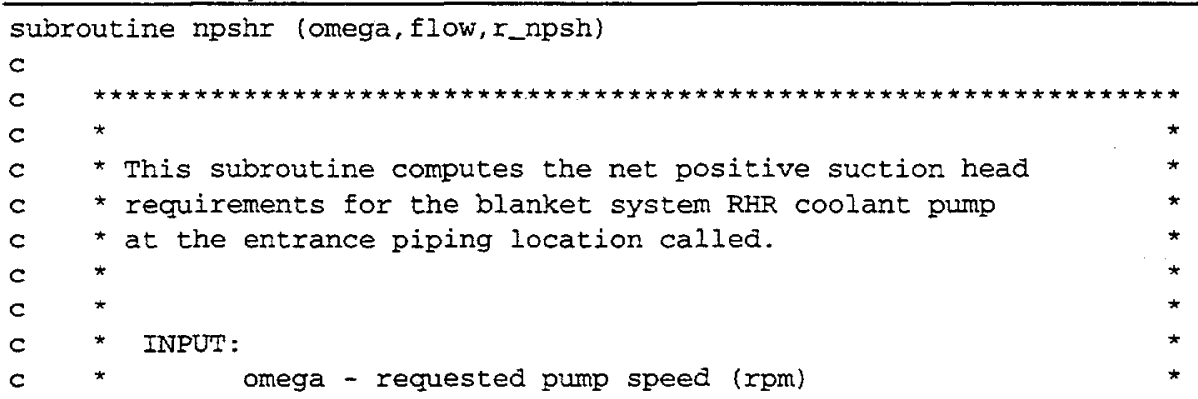




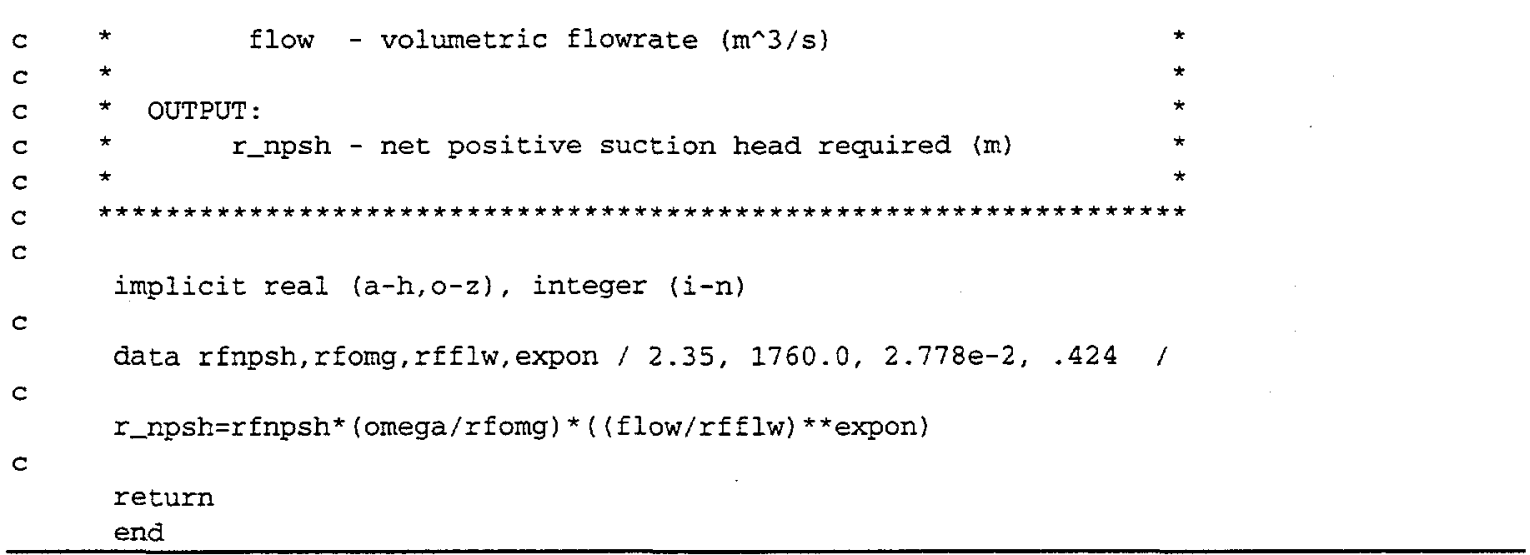

\section{Subroutine pipdlp:}

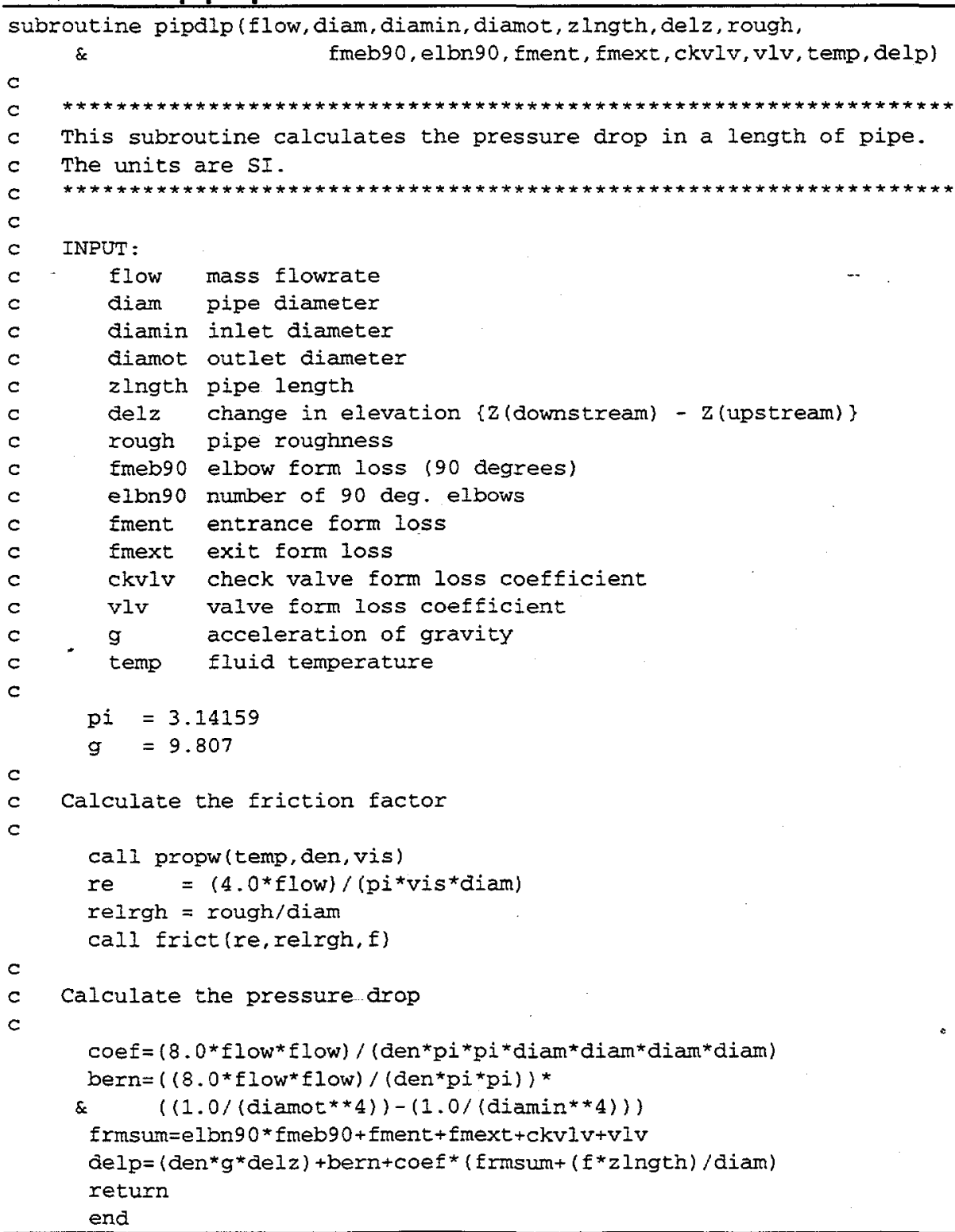




\section{Subroutine pmphc:}

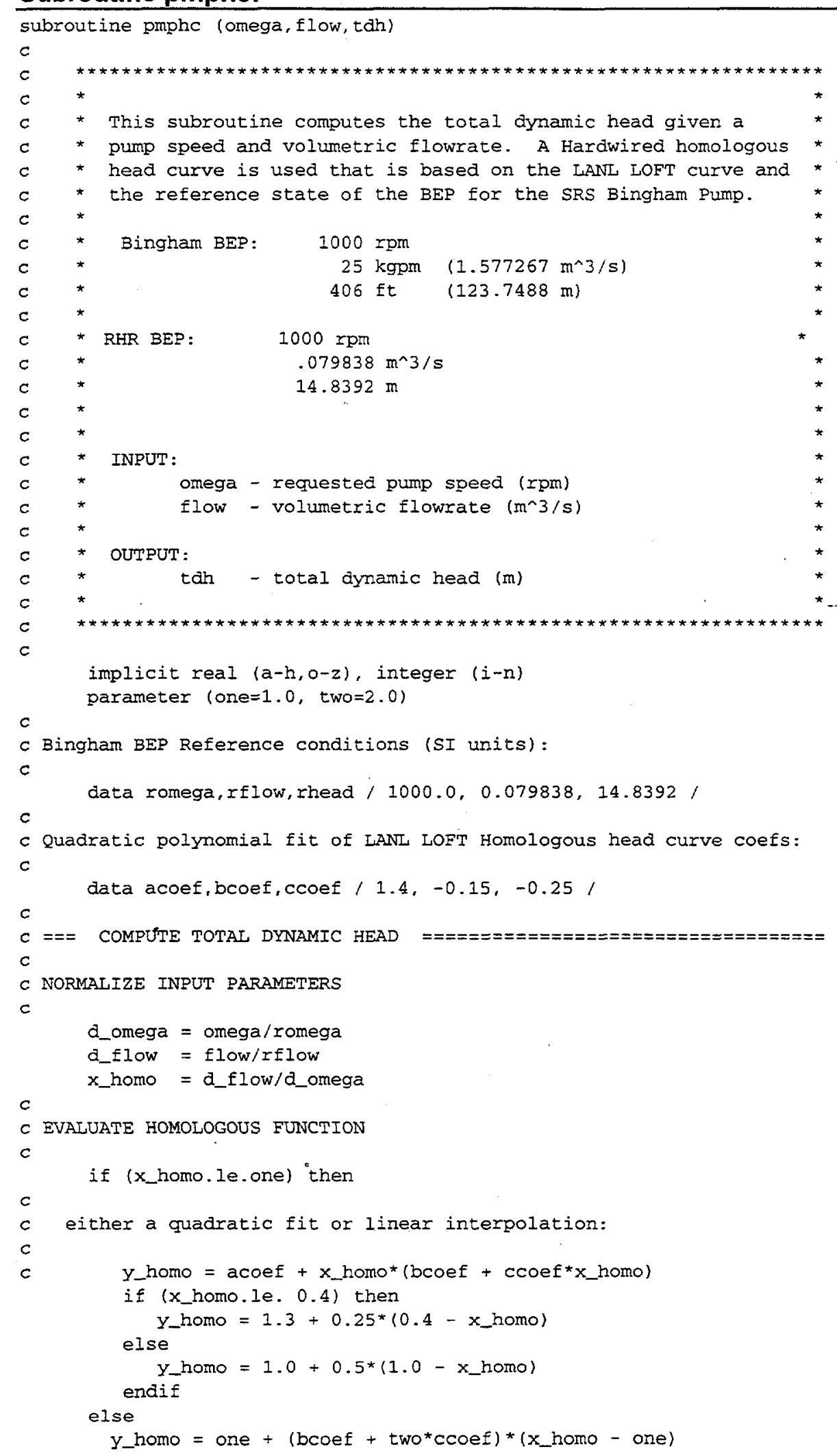


endif

$\mathrm{c}$

C SCALEUP TO THD VALUE

$t d h=y \_h o m o * d \_o m e g a * d \_o m e g a * r h e a d$

$c$

return

end

\section{Subroutine propw:}

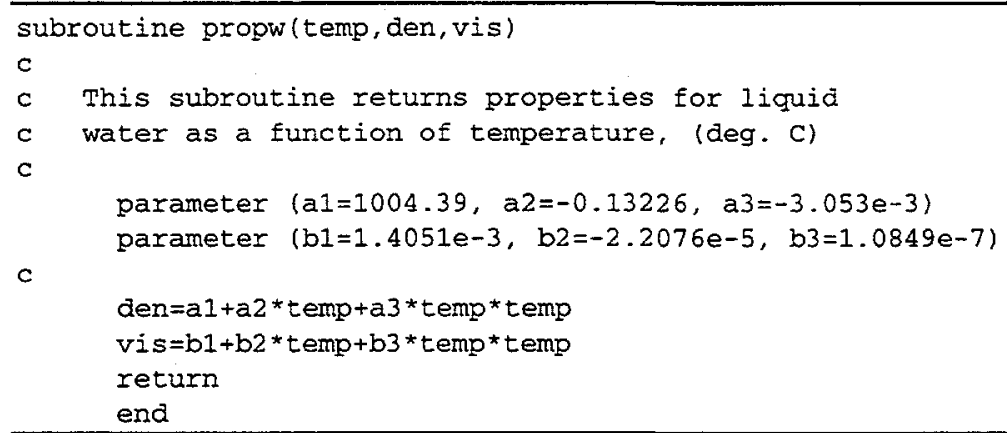

\section{Subroutine psat:}

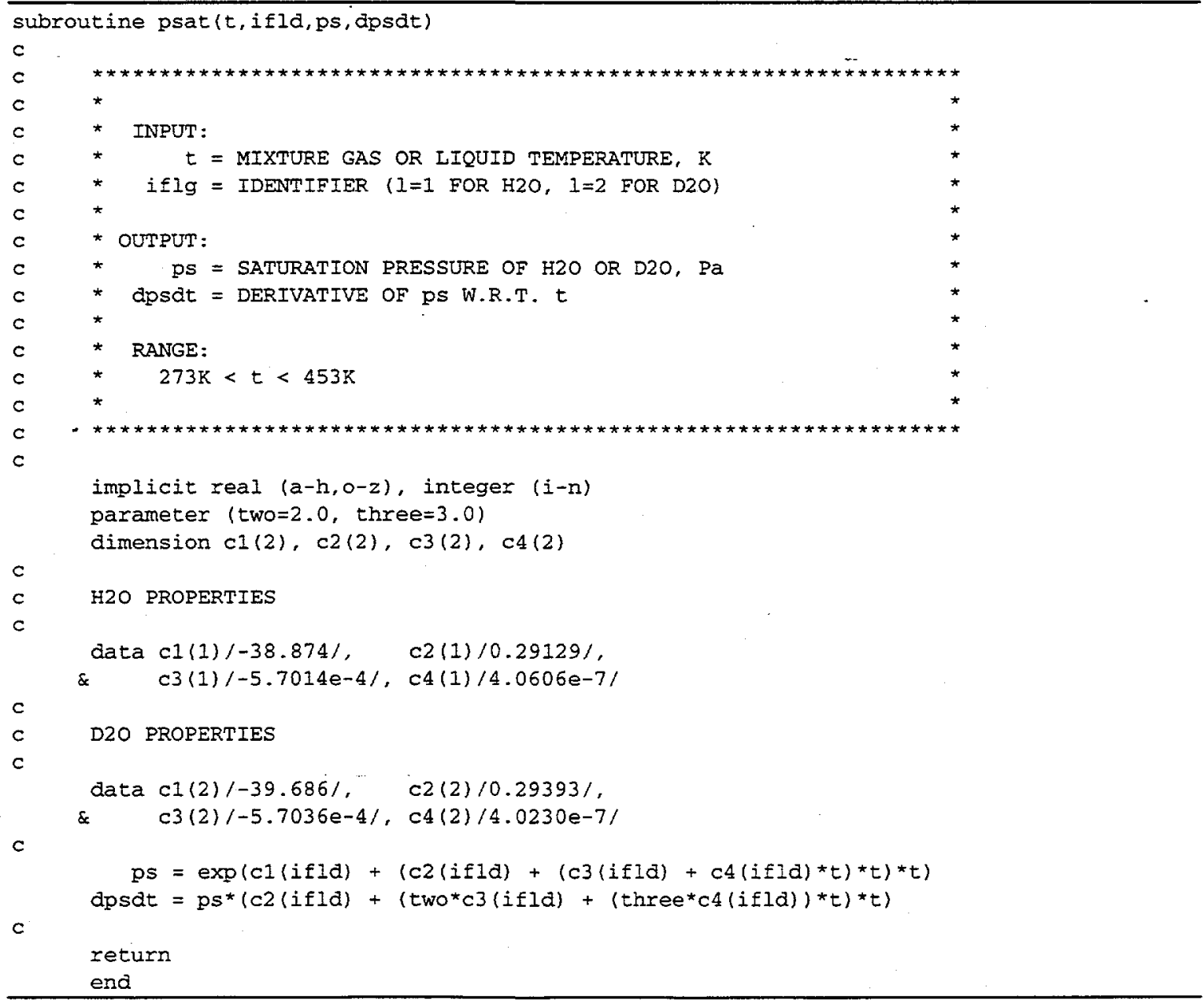




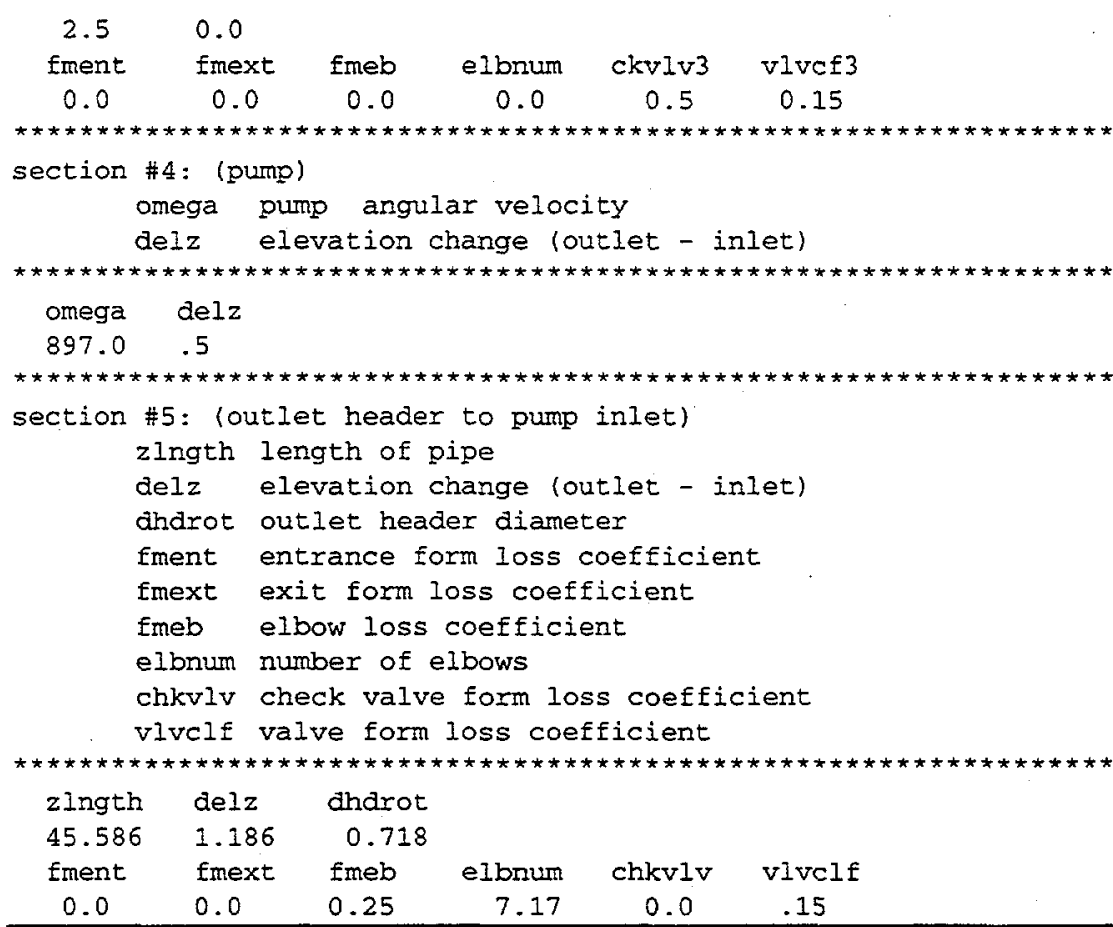

Output File: (pipe6in_4\%.out)

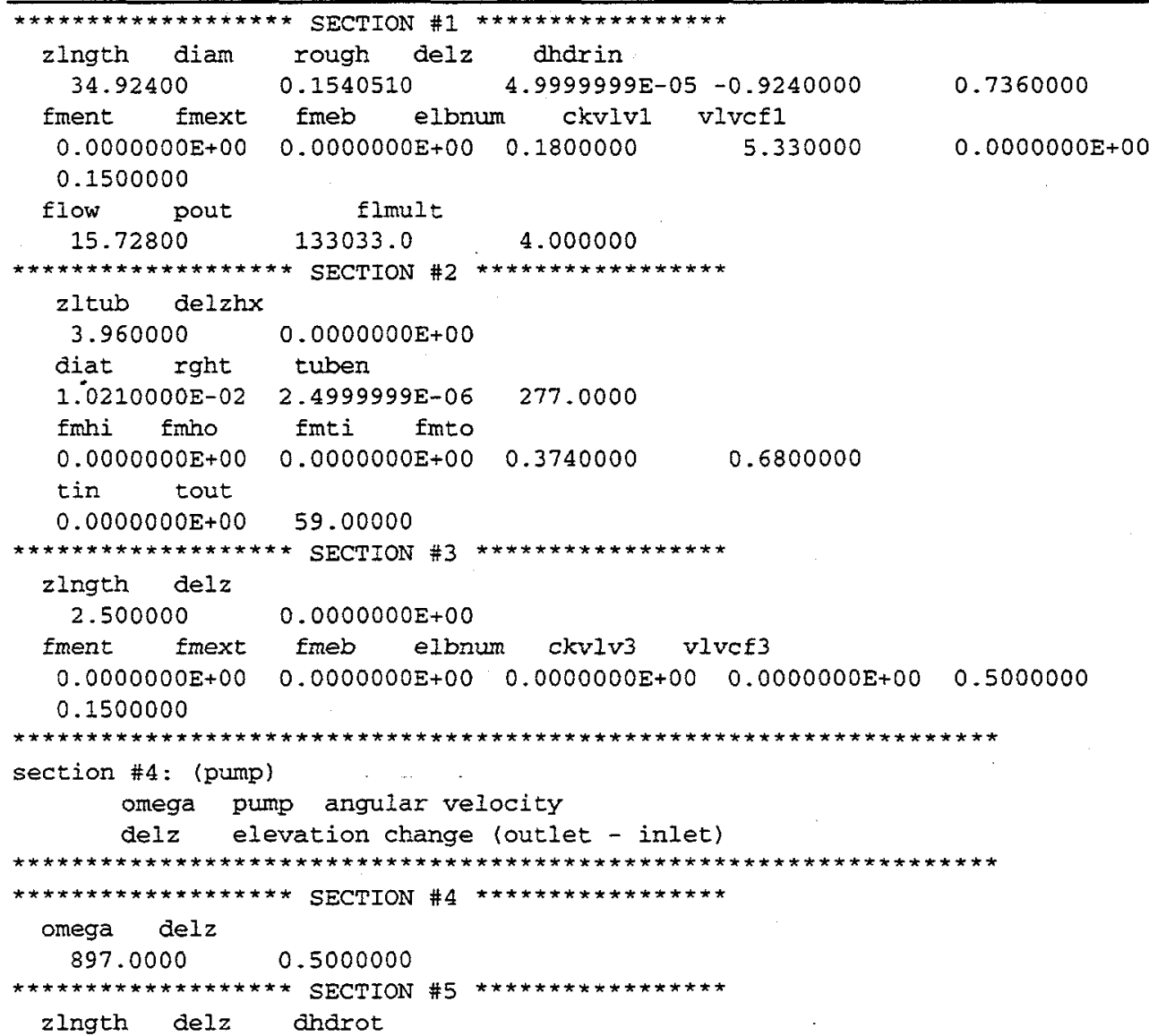




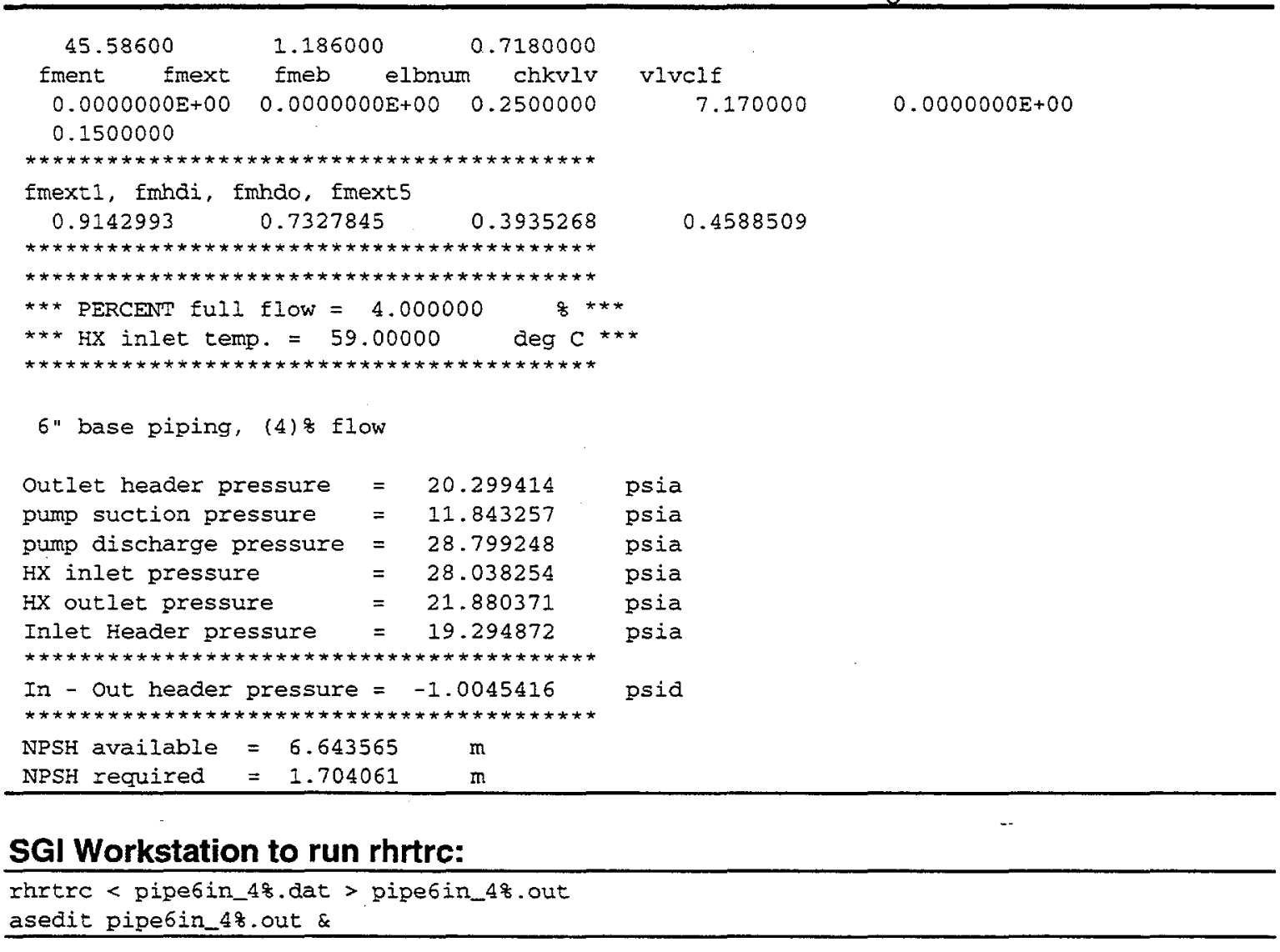

